\title{
Ignition and Burn Control Characteristics of Thermonuclear Plasmas
}

by

\author{
Emmanouil Antony Chaniotakis \\ B.S., Physics, University of Minnesota (1983) \\ M.S., Mechanics, University of Minnesota (1985)
}

Submitted to the Department of Nuclear Engineering in partial fulfillment of the requirements for the degree of

Doctor of Philosophy

at the

MASSACHUSETTS INSTITUTE OF TECHNOLOGY

June 1990

(C) Massachusetts Institute of Technology 1990

\section{Signature redacted}

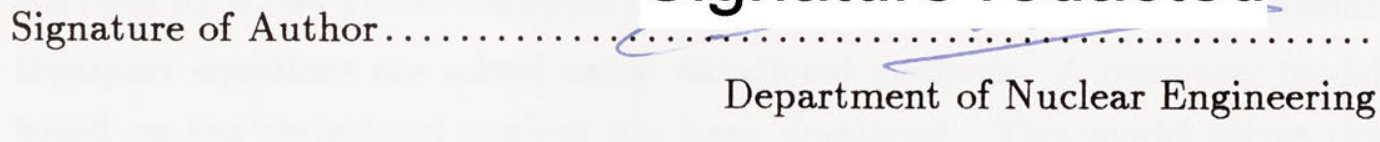

Certified by

June 20, 1990

Signature redacted

Jeffrey P. Freidberg

Professor of Nuclear Engineering

Thesis Supervisor

Certified by

\section{Signature redacted}

Daniel R. Cohn

Senior Research Scientist, Plasma Fusion Center

Thesis Reader

Signature redacted

Accepted by

Allan F. Henry

Chairman, Dentental Gampmittee on Graduate Students OF TECH:Mingy

NOV 131990

LIBRARIES 


\title{
Ignition and Burn Control Characteristics \\ of Thermonuclear Plasmas
}

by

Emmanouil Antony Chaniotakis

\author{
Submitted to the Department of Nuclear Engineering \\ on June 20, 1990, in partial fulfillment of the \\ requirements for the degree of \\ Doctor of Philosophy
}

\begin{abstract}
Achieving the long sought goal of fusion energy requires the attainment of an ignited and controlled thermonuclear plasma. Obtaining an ignited plasma in a tokamak device requires consideration of both the physics of the plasma and the engineering of the machine. With the aide of a completely analytical procedure optimized and ignited tokamaks are obtained under various physics assumptions. These designs show the possible advantage of tokamaks characterized by high $(\sim 4.5)$ aspect ratio, and high $(\sim 15 \mathrm{~T})$ toroidal magnetic field. The control of an ignited plasma is investigated by using auxiliary power modulation. With auxiliary power stable operating points can be created with $Q \sim 50$. Recognizing the need for a fast $1 \frac{1}{2}-\mathrm{D}$ transport model for studying profile effects the plasma transport equations are solved using variational methods. A computer model based on the variational method has been developed. This model solves the $1 \frac{1}{2}-\mathrm{D}$ transport equations very fast with little loss of accuracy.
\end{abstract}

Thesis Supervisor: Jeffrey P. Freidberg

Title: Professor of Nuclear Engineering

Thesis Reader: Daniel R. Cohn

Title: Senior Research Scientist, Plasma Fusion Center 
To Liz 


\section{Acknowledgements}

Many people have, both directly and indirectly, helped me during my education at MIT and I would like to take this opportunity to thank them all.

Since my arrival at MIT two people, Professor Jeff Freidberg and Dr. Dan Cohn, have been very supportive and have, each in their own way, provided both excitement and motivation as well as understanding when personal matters seemed to require more attention than usual.

Professor Jeff Freidberg, my teacher and thesis advisor, has patiently and unselfishly spend many hours discussing plasma physics and engineering. His natural ability to provide the deepest and the clearest understanding of any scientific issue has proven to be an invaluable part of my education. Through his style and teaching ability he has helped me build a strong foundation on which I can base my professional career and I will always be in debt to him.

I am also indebted to Dr. Dan Cohn, for his support since my arrival at MIT. He has given me the opportunity to represent his group at various scientific meetings and as a result I had the opportunity to incorporate a whole new dimension to my graduate education experience. Dr. Cohn has always found the time to listen to my thoughts, and I have gained immensely from his criticism.

Many other people at MIT deserve credit for making my graduate years exciting both scientifically and socially. In particular I would like to thank Dr. Leslie Bromberg for always being able to provide important scientific information. Leslie has always found ways to lighten even the glumiest situation with a style that is only his. Also I would like to thank Professor Dieter Sigmar for acting on my behalf on many occasions, and for always being eager to discuss any issue.

I would also like to thank Dr. Scott Haney with whom I had numerous discussions and exchange of ideas especially with regard to the variational 
formulation of the transport equations. His suggestions, and the always constructive criticism he provides, has helped improve the quality of this work.

Also I would like to thank all the professors who either by teaching a certain class or by just providing information have enhanced my graduate experience. In particular I would like to mention Professors Ian Hutchinson, Mujid Kazimi, John Meyer, and Dr. Marvin Miller of the Nuclear Engineering Department, as well as Professor Eugene Skolnikoff of the Political Science Department whose teachings helped me incorporate the very important aspect of the policy making process to my scientific and engineering training.

My friends at MIT deserve special thanks for providing a stimulating environment in which many interesting physics and social discussions have taken place. Specifically, I would like to thank Kevin Wenzel, Justin Schwartz, Tom Hsu, Bob Witt, Pekka Hakkarainen, Riccardo Betti, John Machuzak, Scott Peng, Bill Stewart, James Wei, Dave Rhee, Jim Crotinger.

Finally, I would like to extend my warmest thanks to my family. Thank you my parents for your constant and unselfish support. The importance you have placed on education has been the stimulus for everything. I want you to know that my education in general and this work in particular has been accomplished as much due to your effort as mine. My brother Nick, thank you for all the good times and for your continuous encouragement.

Antony and Sotiri thank you. You have provided joy in my life and fill my thoughts with dreams. My dear Liz, thank you for your endless love. Your patience, understanding, and your continuous support during the last four years has made it all seem much easier than it actually was.

\section{E.A. Chaniotakis}

Cambridge, June 1990 


\section{Contents}

Acknowledgements

$\begin{array}{ll}\text { List of Figures } & 10\end{array}$

$\begin{array}{ll}\text { List of Tables } & 14\end{array}$

1 Introduction 16

1.1 Design optimization of ignited tokamaks . . . . . . . . 17

1.2 Burn control using auxiliary power modulation . . . . . . 18

1.3 Variational formulation of the transport equation . . . . . 19

1.4 Variational solutions of the plasma transport equations . . . 19

2 Tokamak Plasma Transport and Equilibrium Equations 21

2.1 Introduction . . . . . . . . . . . . . . . . . 21

2.2 Fluid Transport Equations . . . . . . . . . . . . . . 22

2.3 The Ideal MHD Model . . . . . . . . . . . . . . . . . . . 24

2.3 .1 Equation of motion . . . . . . . . . . . . . 24

2.3 .2 Ohm's Law . . . . . . . . . . . . . . . 25

2.4 Toroidal Geometry. Flux Coordinates . . . . . . . . . . 26

2.4.1 Flux Coordinates . . . . . . . . . . . . . . . 27

2.4.2 Force Balance: Grad-Shafranov Equation . . . . . . 31

2.5 Flux Surface Averaged Transport Equations . . . . . . . . 32

2.5.1 Particle Conservation Equation . . . . . . . . . . 32

2.5.2 Energy Conservation Equation . . . . . . . . . . . 34

2.6 Magnetic Diffusion Equation . . . . . . . . . . . 35

2.7 Complete $1 \frac{1}{2}-$ D Plasma Transport Model . . . . . . . . 39 
2.7.1 Transport Equations . . . . . . . . . . . . . . 40

2.7.2 Initial and Boundary Conditions . . . . . . . . . 42

2.7.3 Simplified $1 \frac{1}{2}-$ D Plasma Transport Model . . . . . . 43

2.8 Sources and Sinks for Energy _ . . . . . . . . . 47

2.8.1 Collisional Energy Exchange . . . . . . . . . . 47

2.8 .2 Fusion Power . . . . . . . . . . . . . . . . 48

2.8 .3 Ohmic Heating . . . . . . . . . . . . . . . . . . 49

2.8 .4 Radiation Losses . . . . . . . . . . . . . . 49

2.8.4.1 Bremsstrahlung radiation . . . . . . . 50

2.8.4.2 Synchrontron radiation . . . . . . . . 50

2.8 .5 Auxiliary Power . . . . . . . . . . . . . . . 50

2.9 Fast Equilibration Approximation . . . . . . . . . . 51

2.10 Ohmic Constraint . . . . . . . . . . . . . . 53

2.11 Sources and Sinks for Mass . . . . . . . . . . . . 55

2.12 Zero Dimensional Transport Equations . . . . . . . . . . 55

2.12.1 Zero Dimensional Transport Model of a Thermonuclear Plasma . . . . . . . . . . . . . . . 57

2.12.2 Volume Averaged Sources and Sinks for Energy. . . . 59

2.12.2.1 Ohmic Power Density $\left(p_{\Omega}\right) \ldots \ldots$. . . . 60

2.12.2.2 Alpha Power Density $\left(p_{\alpha}\right) \ldots \ldots 1$

2.12.2.3 Auxiliary Power Density $\left(p_{a}\right) \ldots \ldots 1$

2.12.2.4 Bremsstrahlung Radiation $\left(p_{b}\right) \ldots \ldots 1$

2.12.2.5 Synchrontron Radiation $\left(p_{s}\right) \ldots \ldots$. . . . 62

2.12.2.6 Conduction Losses . . . . . . . . . . . . . 62

2.12 .3 0-D Density Equations . . . . . . . . . . . . 63

2.12.4 Complete 0-D Transport Model . . . . . . . . . . . 64

2.12.5 Energy and Particle Confinement Scalings . . . . . 65

2.12.5.1 Energy Confinement Time . . . . . . . . 65

2.12.5.2 Ohmic Scalings. Neo-Alcator . . . . . . . 66

2.12.5.3 Auxiliary Scalings . . . . . . . . . . . . 66 66

2.12.5.4 Particle Confinement Time . . . . . . . 68

2.13 Conclusions . . . . . . . . . . . . . . 68 
3 Design Optimization of Ignited Tokamaks 69

3.1 Ignition Definition . . . . . . . . . . 71

3.1 .1 Ohmic Ignition . . . . . . . . . . . . . 81

3.1 .2 Auxiliary Power Ignition . . . . . . . . . . . . 83

3.1 .3 Non-Ignited Operation . . . . . . . . . . . . . . 85

3.2 The Minimum Auxiliary Power for Ignition . . . . . . . . 85

3.3 Coupling Physics and Engineering . . . . . . . . 86

3.3.1 Optimized Ignition Physics and Machine Performance 86

3.3 .2 TF Coil Design . . . . . . . . . . . . . 90

3.3.3 OH Coil Design: Volt Second Requirements. . . . . . 94

3.3.3.1 Basic Constraint . . . . . . . . . . . . 94 94

3.3.3.2 Evaluation of $\Delta \psi \ldots \ldots \ldots \ldots$

3.3.3.3 Relate the maximum field $B_{m}$ to the maximum stress $\sigma_{p} \ldots \ldots \ldots \ldots \ldots$

3.3.3.4 Intermediate form of the Volt-Seconds constraint . . . . . . . . . . . 9 97

3.3.3.5 Final form of the Volt-Seconds constraint . 98

3.4 Results . . . . . . . . . . . . . . . . . . . . . 98

3.5 Conclusions . . . . . . . . . . . . . . . . . 102

4 Burn Control Using Auxiliary Power Modulation 107

4.1 Tokamak Thermal Instability and the Need for Burn Control 107

4.2 Mapping the Tokamak Operating Space . . . . . . . . . 114

4.3 Burn Control Issues . . . . . . . . . . . . . . . . . . . . . . 121

4.3.1 Thermal Equilibrium . . . . . . . . . . . . . . . 124

4.3 .2 Stable Operation . . . . . . . . . . . . . . . 124

4.3 .3 Time Scales . . . . . . . . . . . . . . . . . . . 124

4.3.4 Diagnostic and Engineering Issues . . . . . . . . . . . . . . . . . . . . . . . . . . .

4.4 Burn Control Methods . . . . . . . . . . . . . . . 126

4.4 .1 Passive Methods . . . . . . . . . . . . . 126

4.4.1.1 Soft $\beta$ Limit Control . . . . . . . . . . . 126

4.4.1.2 Field Ripple Control . . . . . . . . . . . 126

4.4 .2 Active Methods . . . . . . . . . . . . . . . . 127

$4.4 .2 .1 \quad$ Vary $B_{0} \ldots \ldots \ldots \ldots \ldots \ldots$ 
4.4.2.2 Adiabatic Expansion / Compression . . . . 128

4.4.2.3 Vary the Plasma Density $n_{0} \ldots \ldots$. . . 128

4.4.2.4 Vary the Auxiliary Power $P_{a}$. . . . . . . 129

4.5 Auxiliary Power Control: General Remarks . . . . . . . . 130

4.6 The Complete Burn Control Model . . . . . . . . . . . . 132

4.6 .1 Time Delays . . . . . . . . . . . . . . . . . . . 132

4.6.2 Complete Auxiliary Power Control Model . . . . . . 134

4.7 CIT Burn Control Using Auxiliary Power Modulation . . . . 135

4.7.1 Temperature Evolution . . . . . . . . . . . . 135

4.7 .2 Burn Control . . . . . . . . . . . . . . . . . 141

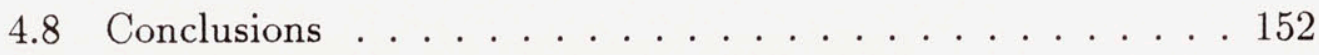

5 Variational Formulation of the Transport Equation 157

5.1 Variational formulation: General Remarks . . . . . . . . 159

5.2 Lagrangian Formulation of Dissipative Systems . . . . . 160

5.3 Variational formulation of the heat equation . . . . . . 164

5.4 Generalized Boundary Conditions . . . . . . . . . . 171

5.5 Test problems . . . . . . . . . . . . . . 172

$5.5 .1 \quad$ Linear Problem . . . . . . . . . . . . . . . . 172

5.5.2 Linear problem with convection . . . . . . . . . . 174

5.5.3 Non-Linear problem with heat pulse . . . . . . . . 174

5.5 .4 Coupled equations. . . . . . . . . . . . . 176

5.6 Variational Formulation of a General System of Transport

Equations. . . . . . . . . . . . . . . 182

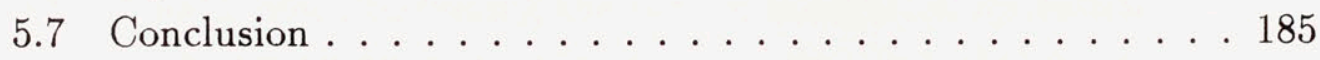

6 Variational Solutions of the Plasma Transport Equations 187

6.1 Introduction . . . . . . . . . . . . . . 187

6.2 Variational Formulation of the Plasma Transport Equations 188

6.2 .1 Lagrangian Formulation . . . . . . . . . . . 190

6.2 .2 Trial Functions . . . . . . . . . . . . . . . . . . 193

6.2.3 System of ODE's to be Solved . . . . . . . . . . 196

6.3 Sawtooth Model . . . . . . . . . . . . . . . . . 204

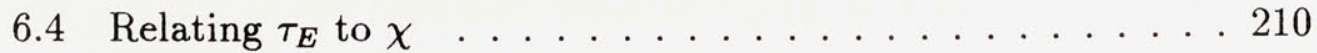

6.5 Solution Procedure . . . . . . . . . . . . . . . . . . 212

6.6 Computer Implementation . . . . . . . . . . . . . . . 214

6.7 Sample results of the $1 \frac{1}{2}-$ D MITra transport code . . . . 214 
7 Summary and Conclusions; Suggestions for future work 224 7.1 Summary . . . . . . . . . . . . . . . . . . 224

7.2 Future Work . . . . . . . . . . . . . . 226

A Coefficients for the 0-D Transport Equations 230

$\begin{array}{ll}\text { Bibliography } & 232\end{array}$ 


\section{List of Figures}

1.1 A plot showing the optimization of $P_{a} / P_{\Omega} \ldots \ldots \ldots$

1.2 A typical test of the variational formulation . . . . . 20

2.1 Cylindrical geometry for axisymmetric toroidal equilibrium . 27

2.2 Countours of constant $\rho$, and $\theta \ldots \ldots \ldots \ldots$

3.1 Illustrative $\dot{T}$ vs $T$ plot with the three equilibrium points $T_{\Omega}$, $T_{B}$, and $T_{R} \ldots \ldots \ldots \ldots \ldots \ldots \ldots \ldots \ldots \ldots \ldots \ldots \ldots \ldots$

$3.2 \dot{T}$ vs $T$ plot illustrating the case of ohmic ignition . . . . 74

$3.3 \dot{T}$ vs $T$ plot illustrating the case of auxiliary power ignition 75

$3.4 \dot{T}$ vs $T$ plot illustrating the case of auxiliary power ignition in a low performance machine . . . . . . . 76

$3.5 \dot{T}$ vs $T$ plot illustrating the case of nonignited operation . . 78

3.6 Typical POPCON plot indicating the Marginal Ignition Ridge

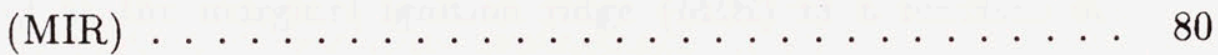

3.7 A plot of the performance paramenet $W$ as a function of the Murakami density factor $N \ldots \ldots \ldots \ldots$

3.8 A plot of the optimum values for $W$ and $N$ as a function of the exponent $\beta$ in Eq. (3.19) . . . . . . . . . . . 84

3.9 A plot of the optimum value of $P_{a} / P_{\Omega}$ as a function of the paraneter $W \ldots \ldots \ldots \ldots \ldots \ldots$

3.10 A toroidal coil model . . . . . . . . . . . . . . . . 91

3.11 The ohmic heating coil model . . . . . . . . . . 96 
3.12 A plot of the quanity $P_{a} / P_{\Omega}$ versus the minor radius $a$, and the total auxiliary power $P_{a}$ versus $a \ldots$. . . . . . . 100

3.13 Clockwise from top are plotted the major radius $R$ the plasma current $I$ and the toroidal magnetic field $B$ as a function of radius $a$

3.14 The optimized performance parameter $B \tau_{E} / R$ as a function of the plasma minor radius . . . . . . . . . . . . . . . . . . . 104

3.15 The optimized parameter $P_{a} / P_{\Omega}$ (top) and the optimized performance parameter $B \tau_{E} / R$ (bottom) as a function of the plasma minor radius corresponding to ITER scaling . . . . . 105

4.1 CIT $\dot{T}$ vs $T$ plot for the parameters shown on Table 4.1 and for zero auxiliary power. . . . . . . . . . . . . . . . . . 108

4.2 CIT $\dot{T}$ vs $T$ plot for the parameters shown on Table 4.1 and for $18 \mathrm{MW}$ of auxiliary power. . . . . . . . . . . . . 109

4.3 CIT plasma operating contours of auxiliary power for Goldston H-mode scaling . . . . . . . . . . . . . . . . . . . . . . 110

$4.4 \mathrm{n}$ - T plot with contours of linear growth rates $(\gamma)$ of plasma temperature corresponding to Fig. 4.3 . . . . . . . . . . . . 111

4.5 Location of the high temperature stable equilibria corresponding to zero auxiliary power under various confinement scalings.113

4.6 $Q$ at the marginal ignition ridge (MIR) as a function of plasma density for Neo-Alcator (solid line), Goldston (dotted line), and Kaye-Goldston (dashed line) confinement scalings

4.7 The marginal ignition ridge (MIR) and the $Q=5$ contour are shown for CIT under Neo-Alcator scaling . . . . . . . 118

4.8 The marginal ignition ridge (MIR) and the $Q=5$ contour are shown for CIT under Goldston H-mode scaling . . . . . 119

4.9 The marginal ignition ridge (MIR) and the $Q=5$ contour are shown for CIT under Kaye-Goldston H-mode scaling . . 120 
4.10 Temperature evolution of an ignited CIT with "small" amount of auxiliary power.

4.11 Temperature evolution of an ignited CIT with "large" amount of auxiliary power.

4.12 Schematic representation of an original system (a), the form of the auxiliary power (b), and the final system indicating the steady state operating temperature $T_{S}$. . . . . . . . . 131

4.13 CIT POP-CON under Goldston H-mode scaling showing the desired final operating point (point A) . . . . . . . . . . . . 137

4.14 Time evolution of temperature for CIT under Goldston Hmode confinement.

4.15 Time evolution of density for CIT under Goldston H-mode confinement.

4.16 Time evolution of plasma powers for CIT under Goldston H-mode confinement. . . . . . . . . . . . . . . . . . . . . . . 140

4.17 The particle source rate required to maintain the density evolution shown in Fig. 4.15 . . . . . . . . . . . . . . . . . . 142

4.18 Time evolution of the thermonuclear $Q$ corresponding to Fig. 4.14 . . . . . . . . . . . . . . . . . . 143

4.19 The e-folding time for the CIT unstable equilibria corresponding to Goldston H-mode confinement . . . . . . . . . . 144

4.20 The e-folding time for the CIT unstable equilibria corresponding to Neo-Alcator confinement . . . . . . . . . 145

4.21 The dependance of the steady state $\mathrm{Q}$ on the maximum temperature deviations stabilized in an ideal system . . . . . . . 147

4.22 Stabilization of an instantaneous $1.5 \mathrm{keV}$ positive temperature deviation for different values of $\tau_{d} \ldots \ldots$. . . . . . . . . . 149

4.23 Evolution of auxiliary power required to stabilize a $1.5 \mathrm{keV}$ positive temperature deviation for various values of $\tau_{d}$. . . 150

4.24 Stabilization of an instantaneous $1.5 \mathrm{keV}$ negative temperature deviation for different values of $\tau_{d} \ldots \ldots$. . . . . . . 151 
4.25 Evolution of auxiliary power required to stabilize a $1.5 \mathrm{keV}$ negative temperature deviation for various values of $\tau_{d} \ldots 153$

4.26 Simulation of a control system characterized a large delay time (solid line) and a small delay time (dashed line) . . . 154

4.27 The maximum temperature deviation to be stabilized as a function of the feedback delay time $\tau_{d} \ldots \ldots \ldots \ldots$

5.1 Complex mechanical system . . . . . . . . . . . . 158

5.2 Trial function test . . . . . . . . . . . . . . 167

5.3 Evaluation of the variational method for a linear problem . . 173

5.4 Evaluation of the variational method for a linear problem with convection . . . . . . . . . . . . 175

5.5 Evaluation of the variational method for a non-linear problem with a heat pulse. . . . . . . . . . . . 177

5.6 Comparison of the profile obtained by a five radial grid point finite differencing method and the variational formulation. . 178

5.7 Evaluation of the variational method for a system of two coupled non-linear heat equations.

6.1 Temperature evolution from the 0-D and MITra transport models

6.2 Temperature evolution from the 0-D and MITra transport models showing the approach to an equilibrium by auxiliary power modulation . . . . . . . . . . . . 217

6.3 Evolution of the thermonuclear $Q$ corresponding to Fig. 6.2 for CIT under Goldston $\mathrm{H}$-mode confinement and for $\chi=$ $C_{\chi} \exp \left[\rho^{2}\right]$ where $C_{\chi}$ is found from Eq. (6.135) . . . . . . 218

6.4 Temperature evolution from the MITra transport model showing the approach to an equilibrium and the control of a negative perturbation by auxiliary power modulation . . . . . 219

6.5 Temperature evolution from the MITra transport model showing the approach to an equilibrium and the control of a positive perturbation by auxiliary power modulation . . . . . 220

6.6 Ohmic temperature evolution with and without sawteeth . . 222

6.7 A plot of the peak temperature during sawtooth activity with a prescribed period corresponding to Fig. 6.6 . . . . . 223 


\section{List of Tables}

2.1 Typical values for the heat exchange time for a deuterium plasma at a temperature of $10 \mathrm{keV} \ldots \ldots \ldots . \ldots 51$

3.1 Critical Parameters for Ohmic Ignition . . . . . . . . . . 81

3.2 Critical Parameters for Auxiliary Power Ignition . . . . . . . 83

3.3 The Parameters $\alpha_{j}$ corresponding to Eq. (3.59) for various confinement scalings . . . . . . . . . . . . . 99

3.4 Summary of the optimized design parameters for Goldston, Neo-Alcator, and ITER scalings . . . . . . . . . . . 102

4.1 CIT Parameters . . . . . . . . . . . . . . . . . . . 112

5.1 Comparison of execution times on a VAX computer for the variational and finite differencing methods for the non-linear problem with heat pulse . . . . . . . . . . 176

5.2 Comparison of execution times on a VAX computer for the variational and finite differencing methods for the problem of coupled non-lineal transport equations. . . . . . . . . 182 


\section{Chapter 1}

\section{Introduction}

The fundamental problem faced by the fusion community over the past 40 years has been the development of a confinement device which can contain the thermonuclear plasma for a sufficiently long time and without major instabilities. The advent of the tokamak $[1,2]$ in the 1960 's provided the best to date confinement scheme for a thermonuclear plasma. With the aide of the tokamak very substantial results have been achieved.

Even though there is general agreement on the importance of the tokamak concept in fusion research, there is no agreement on the particular geometric configuration that a fusion experiment, which is designed to ignite, should have. The design of an ignition experiment is guided by the physics of the thermonuclear plasma and by engineering considerations. The biggest contributor to the uncertainty associated with tokamak design is the incomplete understanding of plasma transport. A strong manifestation of this uncertainty is the plethora of models which attempt to describe both the local and the global plasma transport characteristics.

In order to minimize the uncertainty associated with the predicted performance of a certain tokamak design it is important that extensive scoping studies are done in order to investigate the effect of all relevant theories.

This thesis is primarily concerned with the development of ideas and tools which can be used in order to investigate the behavior of fusion plasmas, and to aide in the design of tokamaks. In particular the following questions are addressed. 
1. How can tokamak designs be developed which are optimized and capable of achieving ignition?

2. How can an ignited plasma be controlled?

3. Is it possible to improve the tools by which tokamak scoping studies are performed?

The above questions are addressed by using the transport models developed on chapter 2. There, by starting with the Fokker-Planck equation, various plasma transport models ranging from the complete fluid model, to the volume averaged (0-D) transport model are developed.

The first two questions are answered by employing the zero dimensional model developed on chapter 2. An affirmative answer to the third equation is obtained by using a variational formulation in order to solve the transport equation.

\subsection{Design optimization of ignited tokamaks}

It is evident from experimental observations that the plasma global energy confinement time decreases with auxiliary power. On the other hand ohmically heated tokamaks report energy confinement scalings which are more reliable and optimistic. Therefore, by minimizing the ratio $P_{a} / P_{\Omega}$, subject to the conditions that the plasma is ignited and that certain engineering considerations are satisfied, optimized designs are obtained under various confinement scalings. For example the ratio $P_{a} / P_{\Omega}$ has a well defined minimum when plotted versus the plasma minor radius $a$, as shown on Fig. 1.1 under Goldston $\tau_{E}$ scaling. By calculating the tokamak parameters at which the ratio $P_{a} / P_{\Omega}$ is minimized, designs are obtained which indicate the possible advantage of high $(\sim 15 T)$ magnetic field, and high (4.5) aspect ratio. 


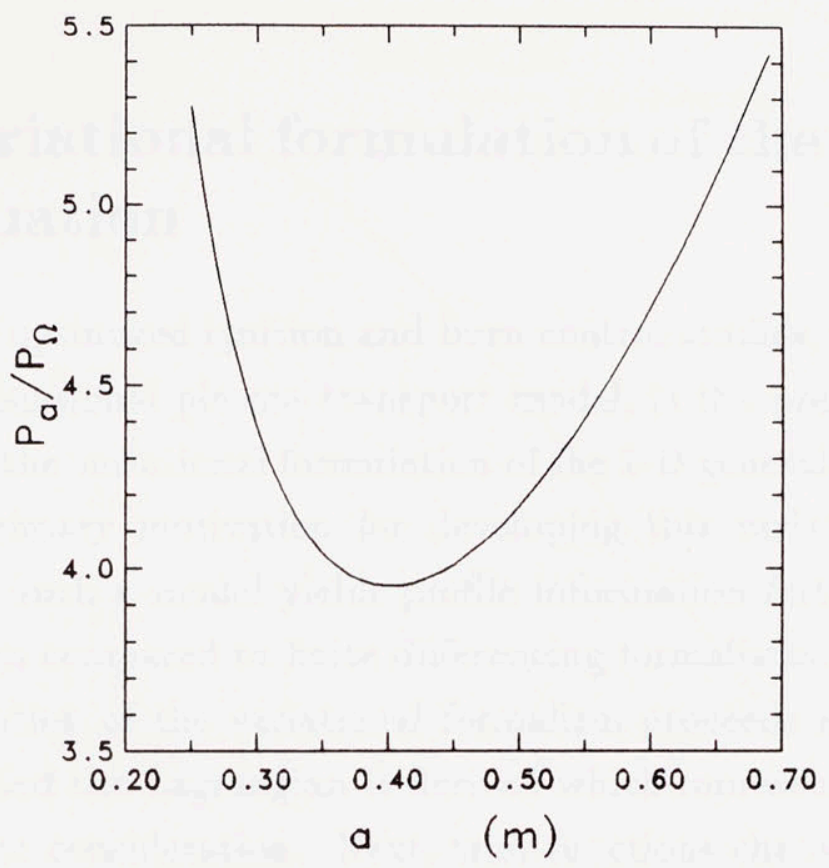

Figure 1.1: The ratio of the auxiliary power to the ohmic power is plotted as a function of minor radius $a$. Note the well defined minimum that the $P_{a} / P_{\Omega}$ assumes at a certain $a$.

\subsection{Burn control using auxiliary power mod- ulation}

The ignited tokamak designs just discussed will be operating in a regime characterized by unstable equilibria. In this situation some means of controlling the plasma at the desired equilibrium must be provided. For the first generation ignited tokamak it is important that the most reliable method of burn control is used. By comparing the various burn control methods that have been suggested over the years, burn control via auxiliary power modulation appears to be the most reliable.

By modulating the auxiliary power supplied to the plasma stable equilibria are created and fluctuations about these equilibria can be stabilized by dynamically altering the auxiliary power supplied to the plasma. The equilibria created by auxiliary power modulation are characterized by finite thermonuclear $Q$ values since there is a small amount of auxiliary power that is continuously supplied to the plasma. 


\subsection{Variational formulation of the transport equation}

Following the optimized ignition and burn control studies, which are based on a zero dimensional plasma transport model, is the presentation of the basic ideas in the variational formulation of the 1-D general transport equation. The primary motivation for developing this variational transport model is that such a model yields profile information fast, and with high accuracy, when compared to finite differencing formalisms.

The derivation of the variational formalism proceeds as follows. First a function called the Lagrangian is derived which represents the transport equation under consideration. Next, trial functions characterized by certain unknown parameters "variational parameters" are substituted into the Lagrangian. In turn the variational solution to the transport equation is found by estimating the value of the variational parameters which make the Lagrangian stationary.

The variational method for the solution of the transport equation has been estimated to reduce the required computational time by a factor of 10 50 over standard finite differencing techniques. Furthermore, by choosing the right trial functions the accuracy of the method is very good (again when compared with the exact solution). This is shown on Fig. 1.2 where a profile obtained by the variational formulation is compared to the exact solution.

\subsection{Variational solutions of the plasma trans- port equations}

Having developed and tested the variational formulation for the transport equation, the next goal is to develop a complete plasma transport model. This is accomplished in chapter 6 where the $1 \frac{1}{2}-\mathrm{D}$ plasma transport model developed on chapter 2 is formulated variationally. Also, a sawtooth model 


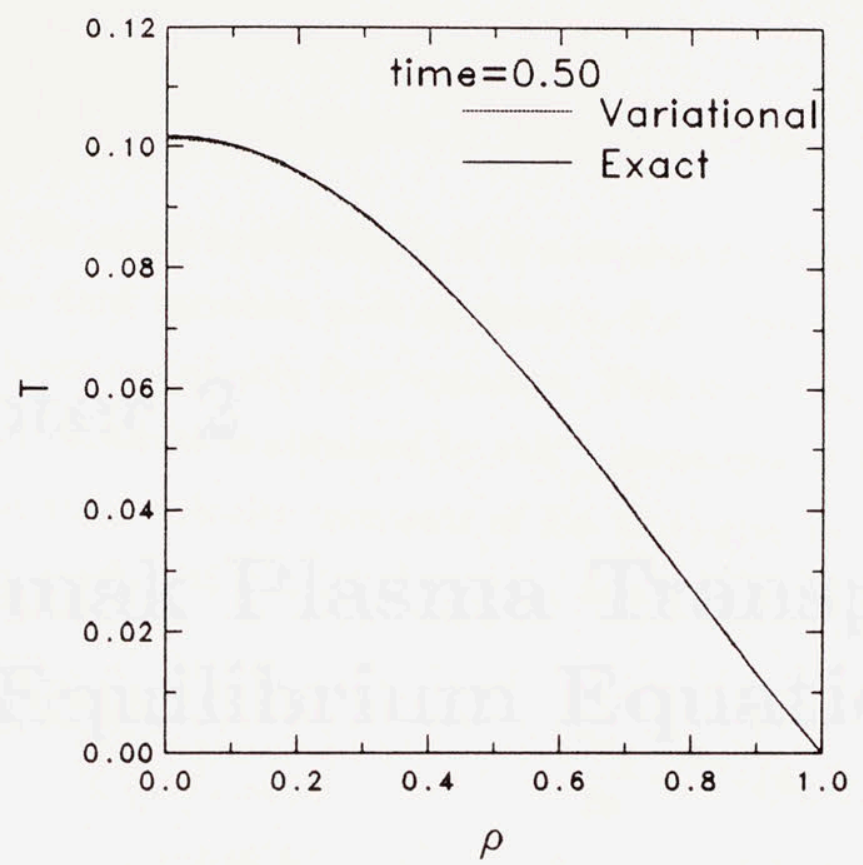

Figure 1.2: Comparison of the results obtained from the variational and the exact solution of the transport equation.

is developed, and the issues related to the connection between the global energy confinement time $\tau_{E}$ and the local diffusion coefficients are discussed.

The formulation is incorporated into a computer code called MITra which solves the $1 \frac{1}{2}-\mathrm{D}$ time dependent plasma transport equations. This code is in turn used to study some fundamental ignition and burn control issues of the CIT tokamak. 


\section{Chapter 2}

\section{Tokamak Plasma Transport and Equilibrium Equations}

\section{$2.1 \quad$ Introduction}

Plasmas are composed of a large number of charged particles of different species. For example, in a tokamak plasma the main species are: electrons, hydrogen, deuterium, tritium, alpha particles, as well as many other ions characterized by higher atomic numbers, the so called impurities. The behavior of such a plasma is described in terms of the particle motions with the aide of the distribution function $f(\vec{x}, \vec{v}, t)$. The distribution function $f_{j}$ is a function of seven variables and it gives a measure of the probability density for the particles of species $j$ in the $(\vec{x}, \vec{v})$ phase space.

The time evolution of the distribution function $f_{j}(\vec{x}, \vec{v}, t)$ of species, $j$, in a multiple species plasma is given by the (Vlasov) Fokker -Planck equation

$$
\frac{\partial f_{j}}{\partial t}+\vec{v} \cdot \nabla f_{j}+\frac{e_{j}}{m_{j}}(\vec{E}+\vec{v} \times \vec{B}) \cdot \frac{\partial f_{j}}{\partial \vec{v}}=C_{j}+S_{j}
$$

where $e_{j}$ is the charge and $m_{j}$ the mass of particles of species $j, \vec{E}$ and $\vec{B}$ are respectively the macroscopic electric and magnetic fields. $C_{j}$ is the Coulomb collision operator and $S_{j}$ represents any external particle source or sink of species $j$. 


\subsection{Fluid Transport Equations}

In general, for many applications, it is adequate to describe the plasma in terms of the fluid variables such as density, fluid velocity and temperature which are functions of only four variables. This reduction in the number of independent variables is obtained by taking moments of Eq. (2.1).

The first three velocity moments of Eq. (2.1) give the fluid expressions for the conservation of mass, momentum, and energy respectively.

$$
\begin{aligned}
\frac{\partial n_{j}}{\partial t}+\nabla \cdot\left(n_{j} \vec{u}_{j}\right) & =S_{n j} \\
n_{j} m_{j}\left(\frac{d \vec{u}_{j}}{d t}\right)-e_{j} n_{j}\left(\vec{E}+\vec{u}_{j} \times \vec{B}\right)+\nabla \cdot \Pi_{j} & =\mathbf{R}_{j} \\
\frac{3}{2} \frac{\partial\left(n_{j} T_{j}\right)}{\partial t}+\frac{3}{2} \nabla \cdot\left(n_{j} T_{j} \vec{u}_{j}\right)+n_{j} T_{j} \nabla \cdot \vec{u}_{j}+\Pi_{j}: \nabla \vec{u}_{j} & =Q_{j} \\
-\nabla \cdot \vec{q}_{j} & +S_{E j}
\end{aligned}
$$

In the above equations the various fluid variables are: $[3]$

The density $n_{j}$ of species $j$,

$$
n_{j} \equiv \int f_{j} d \vec{v}
$$

the flow velocity, $u_{j}$ of species $j$

$$
\vec{u}_{j} \equiv \int \vec{v} f_{j} d \vec{v}
$$

the particle source rate,

$$
S_{n j} \equiv \int S_{j} d \vec{v}
$$

the total pressure tensor, 


$$
\Pi_{\mathbf{j}} \equiv \int m_{j}\left(\vec{v}-\vec{u}_{j}\right)\left(\vec{v}-\vec{u}_{j}\right) f_{j} d \vec{v}
$$

the friction force,

$$
\mathbf{R}_{\mathbf{j}} \equiv \int m_{j}\left(\vec{v}-\vec{u}_{j}\right) C_{j} d \vec{v}
$$

the temperature of species $j$,

$$
T_{j} \equiv \frac{p_{j}}{n_{j}}
$$

where $p_{j}$ is the scalar pressure

$$
p_{j} \equiv \int \frac{m_{j}}{3}\left|\vec{v}-\vec{u}_{j}\right|^{2} f_{j} d \vec{v}
$$

the heat flux,

$$
\vec{q}_{j} \equiv \frac{1}{2} \int m_{j}\left(\vec{v}-\vec{u}_{j}\right)\left|\vec{v}-\vec{u}_{j}\right|^{2} f_{j} d \vec{v}
$$

the heat generated due to collisions between unlike particles,

$$
Q_{j} \equiv \frac{1}{2} \int m_{j}\left|\vec{v}-\vec{u}_{j}\right|^{2} C_{j} d \vec{v}
$$

and the energy source,

$$
S_{E j}=\frac{1}{2} \int m_{j}|\vec{v}|^{2} S_{j} d \vec{v}
$$

In Eqs. $(2.2-2.4)$, all the fluid variables are functions of space, $\vec{x}$, and time, $t$, (i.e. four independent variables). However, in the study of transport in the toroidal tokamak geometry, it is possible, by choosing an appropriate coordinate system, and considering the behavior of the plasma in the various directions, to reduce the number of independent variables to two, one for the space and one for the time. A summary of the basic assumptions and procedures necessary for this reduction in the number of independent variables is developed in the next two sections. 


\subsection{The Ideal MHD Model}

The hydrodynamic model is useful in describing low frequency phenomena because it is then valid to neglect the electron inertia and to assume that the plasma remains neutral.[4,5] Starting with Eqs.(2.2 - 2.4) the main steps for the derivation of the MHD equations are presented in this section.

\subsubsection{Equation of motion}

The net force balance equation for the plasma is obtained by adding Eq. (2.4) over all species and neglecting the electron inertia

$$
\sum_{j} m_{j} n_{j} \frac{d \vec{u}_{j}}{d t}-\vec{J} \times \vec{B}+\sum_{j} \nabla p_{j}=-\nabla \cdot \sum_{j}\left(\mathcal{P}_{j}\right)
$$

The current density is

$$
\vec{J}=\sum_{j} e_{j} n_{j} \vec{u}_{j}
$$

and $\mathcal{P}_{j}$ is the anisotropic part of the pressure tensor $\Pi_{j}$

$$
\mathcal{P}_{j} \equiv \Pi_{j}-p_{j} \mathbf{I}
$$

where $\mathrm{I}$ represents the identity tensor and $p_{j}$ is the scalar pressure tensor. Note that the sum of the friction forces $\sum_{j} \mathbf{R}_{j}=0$, due to momentum conservation in Coulomb collisions, and that the electric force acting on the plasma is zero due to the quasineutral approximation $\sum_{j} e_{j} n_{j}=0$.

In the development of the MHD model the typical time scale of interest corresponds to the ion thermal transit time $a / V_{T_{i}}$, where $a$ is the macroscopic dimension of the plasma (the length scale of interest). During the time $a / V_{T_{i}}$ a certain number of collisions occur in the plasma between the various plasma species. For a valid fluid treatment it is required that the plasma is collision dominated. This collision dominated assumption 
assures that the distribution functions of the plasma species are nearly Maxwellian. For the plasma ions the dominant collision mechanism is due to ion-ion interactions characterized by a collision time $\tau_{i i}$. Furthermore, the plasma electrons become Maxwellian by colliding either with ions or with other electrons, since $\tau_{e e} \sim \tau_{i e}$.[4] Another requirement for the collisiondominated theory to be valid is that the macroscopic plasma length scale $a$ be much longer than the mean free path (Debye length $\lambda$ ) for each species (i.e. $\lambda \ll a$ ). In general the conditions for a collision dominated plasma are given by $[4]$

$$
\frac{V_{T_{j}} \tau_{j j}}{a} \ll 1
$$

By considering the relative magnitude of $\nabla \cdot \mathcal{P}$ to $\nabla p$ it can be shown that [4]

$$
\left|\nabla \cdot \mathcal{P}_{j} / \nabla p\right| \sim V_{T_{j}} \tau_{j j} / a \ll 1
$$

Therefore Eq. (2.15) reduces to

$$
\sum_{j} m_{j} n_{j} \frac{d \vec{u}_{j}}{d t}-\vec{J} \times \vec{B}+\sum_{j} \nabla p_{j}=0
$$

\subsubsection{Ohm's Law}

In processes in which all quantities vary slowly in time (no significant changes in one electron ion collision time) [3] the electron inertia may be neglected and the the electron momentum balance becomes.

$$
\vec{E}+\vec{u} \times \vec{B}=\frac{1}{e n}\left(\vec{J} \times B-\nabla p_{e}-\nabla \cdot \mathcal{P}_{e}+\mathbf{R}_{e}\right)
$$

From Eq. (2.19) it is clear that the $\nabla \cdot \mathcal{P}_{\boldsymbol{e}}$ term is negligible compared to $\nabla p_{e}$ term. Furthermore, from Eq. (2.20) it follows that the $\nabla p_{e}$ term is comparable to the $\vec{J} \times \vec{B}$ term. By comparing the $\nabla p_{e}$ term with the $\vec{u} \times \vec{B}$ it gives 


$$
\frac{\left|\nabla p_{e} / e n\right|}{|\vec{u} \times B|} \sim \frac{\rho_{L i}}{a}
$$

where $\rho_{L i}$ is the ion Larmor radius. By assuming that $\rho_{L i} / a \ll 1$ the electron momentum equation reduces to

$$
\vec{E}+\vec{u} \times \vec{B}=\frac{1}{e n} \mathbf{R}_{e}
$$

The term $R_{e}$ represents the friction force between the electrons and the other plasma species. The major contribution of $\mathbf{R}_{\boldsymbol{e}}$ is the electrical resistivity and can be written as[3]

$$
\frac{1}{e n} \mathbf{R}_{e} \sim \eta \vec{J}
$$

where $\eta$ is the electrical resistivity of the plasma.

Finally, the electric field $\vec{E}$, the magnetic field $\vec{B}$, and the plasma current $\vec{J}$ are given by the low frequency Maxwell equations.

$$
\begin{aligned}
\nabla \times \vec{E} & =-\frac{\partial \vec{B}}{\partial t} \\
\nabla \times \vec{B} & =\mu_{0} \vec{J} \\
\nabla \cdot \vec{B} & =0
\end{aligned}
$$

\subsection{Toroidal Geometry. Flux Coordinates}

The local properties of the thermodynamic variables $n_{j}$, and $T_{j}$ can be described in terms of a fixed cylindrical orthogonal system $\vec{x} \equiv(R, \phi, Z)$ as illustrated in Fig. 2.1.

The analysis is based on the assumption of toroidal axisymmetry which implies that $\partial S / \partial \phi=0$, where $S$ is any scalar. [4] 


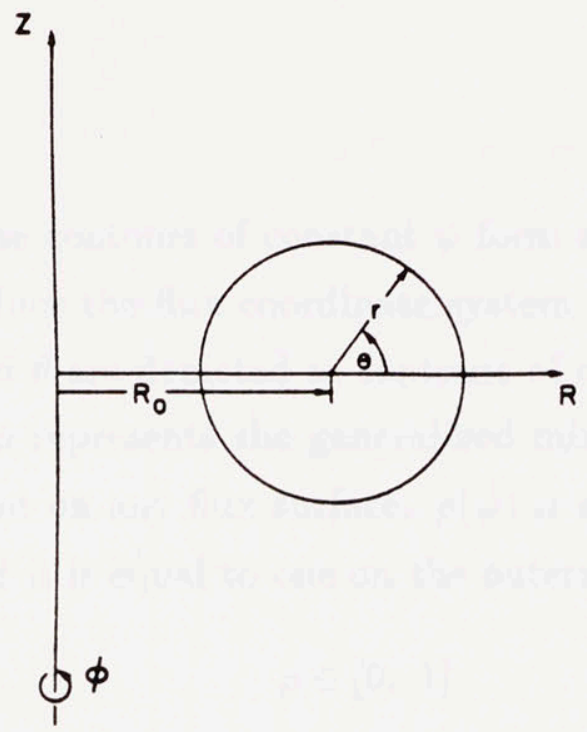

Figure 2.1: Cylindrical geometry for axisymmetric toroidal equilibrium

The purpose of this work is to obtain solutions to the transport equations on the time scale governed by cross field transport and resistive diffusion. This time scale is long compared to the time that Alfvén waves act to equilibrate plasma forces imbalances. Therefore, the inertia term in Eq. (2.20) may be neglected and the plasma is confined by a magnetic field $\vec{B}$ according to the static set of equations.

$$
\begin{aligned}
\nabla P & =\vec{J} \times \vec{B} \\
\nabla \times \vec{B} & =\mu_{0} \vec{J} \\
\nabla \cdot \vec{B} & =0
\end{aligned}
$$

where $P$ denotes the total plasma pressure, and $\vec{J}$ is the plasma current density.

\subsubsection{Flux Coordinates}

In axisymmetric systems, and by virtue of Eq. (2.30), a stream function $\psi$ for the poloidal magnetic field may be introduced.[4] In a system like the 
tokamak where the contours of constant $\psi$ form nested toroidal surfaces it is useful to introduce the flux coordinate system $(\rho, \theta, \phi)$. In Fig. 2.2 the coordinates $\rho$, and $\theta$ are depicted as contours of constant $\rho$, and $\theta$.

The variable $\rho$ represents the generalized minor radius $\rho$ which is, by definition, constant on any flux surface. $\rho(\psi)$ is normalized to zero on the magnetic axis and it is equal to one on the outermost flux surface.

$$
\rho \in[0,1]
$$

Since there is an one-to-one correspondence between a given value of $\rho$ and a particular flux surface, $\rho$ is a flux surface label.

The variable $\theta$ in the flux coordinate system is an angle like variable varies from 0 to $2 \pi$ along the poloidal direction around the flux surface $\rho$. The coordinate $\phi$ (toroidal angle) is the same in both the $(R, \phi, Z)$ and $(\rho, \theta, \phi)$ coordinate systems and $0 \leq \phi \leq 2 \pi$.

The Jacobian of the transformation from the fixed frame $(R, \phi, Z)$ to the flux coordinates $(\rho, \theta, \phi)$ is given by

$$
\begin{aligned}
J & =[(\nabla \rho \times \nabla \theta) \cdot \nabla \phi)]^{-1} \\
& =R\left[\frac{\partial R}{\partial \theta} \frac{\partial Z}{\partial \rho}-\frac{\partial R}{\partial \rho} \frac{\partial Z}{\partial \theta}\right]
\end{aligned}
$$

In general the vectors $\nabla \rho$ and $\nabla \theta$ need not be orthogonal over a particular non-circular flux surface. The following relationships are valid for the vectors $\nabla \rho, \nabla \theta$, and $\nabla \phi$.

$$
\begin{aligned}
& \nabla \rho \cdot \nabla \theta \neq 0 \\
& \nabla \theta \cdot \nabla \phi=0 \\
& \nabla \rho \cdot \nabla \phi=0 \\
& \nabla \phi \cdot \nabla \phi=\frac{1}{R^{2}}
\end{aligned}
$$




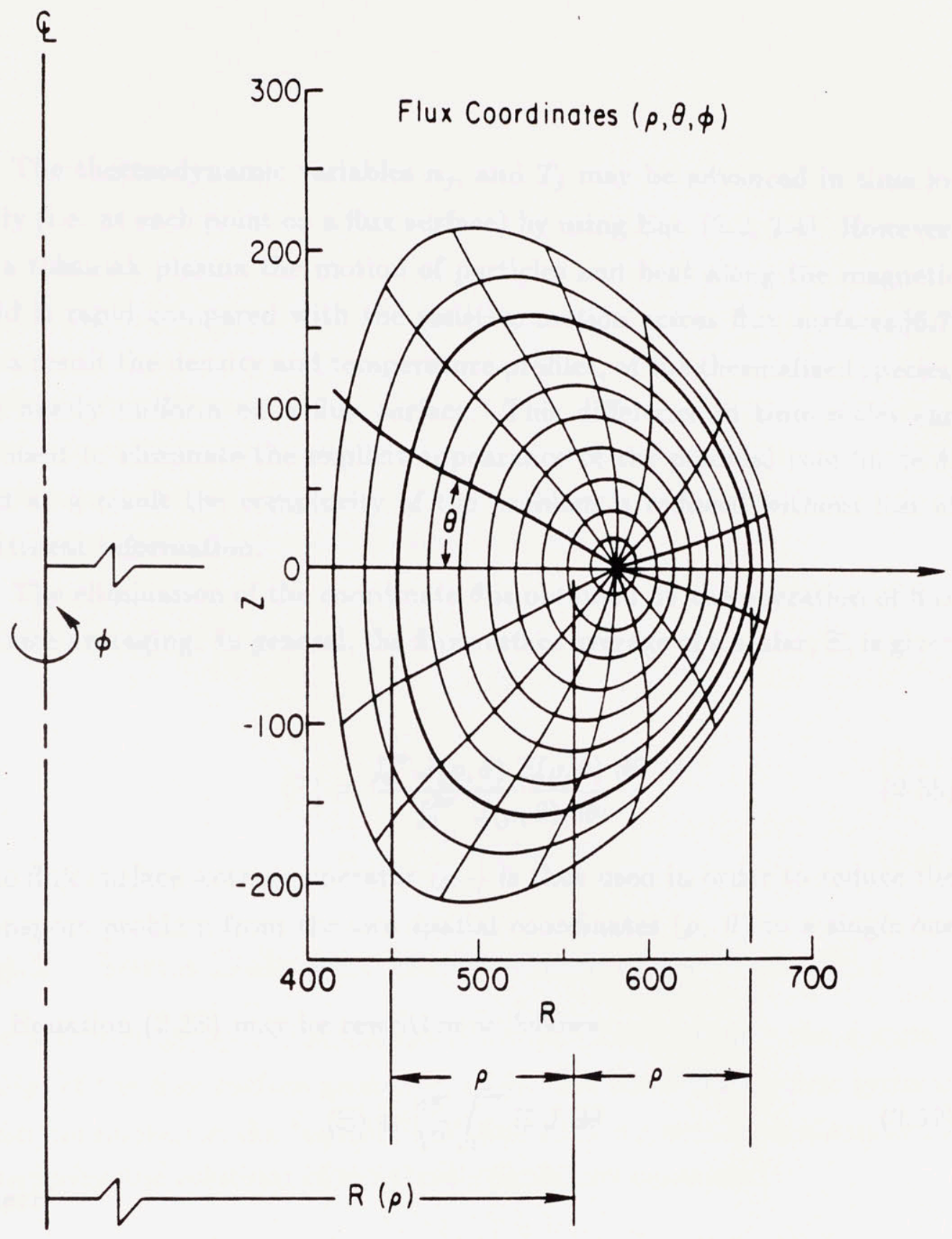

Figure 2.2: Countours of constant $\rho$, and $\theta$ 
The thermodynamic variables $n_{j}$, and $T_{j}$ may be advanced in time locally (i.e. at each point on a flux surface) by using Eqs. $(2.2,2.4)$. However, in a tokamak plasma the motion of particles and heat along the magnetic field is rapid compared with the resistive motion across flux surfaces. $[6,7]$ As a result the density and temperature profiles, of the thermalized species, are nearly uniform on a flux surface. This difference in time scales can be used to eliminate the explicit appearance of the poloidal coordinate $\theta$, and as a result the complexity of the problem is reduced without loss of pertinent information.

The elimination of the coordinate $\theta$ is obtained by the operation of flux surface averaging. In general, the flux surface average of a scalar, $\Xi$, is given by

$$
\langle\Xi\rangle \equiv \frac{\int_{0}^{2 \pi} J(\rho, \theta) \Xi(\rho, \theta) d \theta}{\int_{0}^{2 \pi} J(\rho, \theta) d \theta}
$$

The flux surface average operator $\langle\cdots\rangle$ is thus used in order to reduce the transport problem from the two spatial coordinates $(\rho, \theta)$ to a single one $(\rho)$.

Equation (2.38) may be rewritten as follows

$$
\langle\Xi\rangle \equiv \frac{2 \pi}{V^{\prime}} \int_{0}^{2 \pi} \Xi J d \theta
$$

where

$$
V^{\prime} \equiv \frac{\partial V}{\partial \rho}=2 \pi \int_{0}^{2 \pi} J d \theta
$$

Often, during the development of the flux averaged transport equations the flux average of the divergence of a vector, $\langle\nabla \cdot \vec{A}\rangle$, is required. From Eq. (2.39) we obtain

$$
\langle\nabla \cdot \vec{A}\rangle=\frac{2 \pi}{V^{\prime}} \int_{0}^{2 \pi}(\nabla \cdot \vec{A}) J d \theta
$$


where $\nabla \cdot \vec{A}$ is given by

$$
\nabla \cdot \vec{A}=\frac{1}{J}\left[\frac{\partial}{\partial \rho}\left(J|\nabla \rho| A_{\rho}\right)+\frac{\partial}{\partial \theta}\left(\frac{R}{|\nabla \rho|} A_{\theta}\right)+\frac{\partial}{\partial \phi}\left(\frac{A_{\phi} J}{R}\right)\right]
$$

However, due to assumption of axisymmetry the third term on the right hand side of the above equation is zero. Furthermore, due to periodicity conditions in the poloidal angle $\theta$ the second term on the right hand side of the above equation integrates to zero. Therefore,

$$
\begin{aligned}
\langle\nabla \cdot \vec{A}\rangle & =\frac{2 \pi}{V^{\prime}} \int_{0}^{2 \pi} \frac{\partial}{\partial \rho}\left(J|\nabla \rho| A_{\rho}\right) d \theta \\
& =\frac{2 \pi}{V^{\prime}} \frac{\partial}{\partial \rho} \int_{0}^{2 \pi} J(\vec{A} \cdot \nabla \rho) d \theta \\
& =\frac{1}{V^{\prime}} \frac{\partial}{\partial \rho}\left[V^{\prime}\langle\vec{A} \cdot \nabla \rho\rangle\right]
\end{aligned}
$$

\subsubsection{Force Balance: Grad-Shafranov Equation}

The flux average operation indicated by Eq. (2.39) requires the determination of the flux surface geometry, $\psi(\vec{x})$. For the axisymmetric systems under consideration the "equilibrium" flux surface geometry is obtained by considering the solutions of the Grad - Shafranov equation.

$$
\begin{aligned}
\Delta^{*} \psi & =-\mu_{0} R J_{\phi} \\
& =-\mu_{0} R^{2} P^{\prime}(\psi)-F(\psi) F^{\prime}(\psi)
\end{aligned}
$$

subject to the boundary condition

$$
\psi\left(S_{p}\right)=0
$$

where $S_{p}$ denotes the plasma surface, and the constraint that the flux function $\psi$ remains regular at the magnetic axis. $J_{\phi}$ is the toroidal component of 
the plasma current, and $P(\psi)$ and $F(\psi)$ are "free" functions relating to the pressure and toroidal field profiles. The operator $\left({ }^{\prime}\right)$ denotes differentiation of a function with respect to its argument.

The operator $\Delta^{*}$ is defined

$$
\begin{aligned}
\Delta^{*} \psi & \equiv R^{2} \nabla \cdot\left(\frac{\nabla \psi}{R^{2}}\right) \\
& =R \frac{\partial}{\partial R}\left(\frac{1}{R} \frac{\partial}{\partial R}\right)+\frac{\partial^{2} \psi}{\partial Z^{2}}
\end{aligned}
$$

Having introduced the concept of flux surface average, the next step is to reduce the three dimensional fluid Eqs. $(2.2,2.4)$ to the more useful one dimensional flux surface averaged equations.

\subsection{Flux Surface Averaged Transport Equa- tions}

The flux surface averaged transport equations are obtained by performing the "averaging" operation (Eq. (2.39)) of the transport equations $(2.2,2.4)$ over the flux surfaces obtained by solving the Grad-Shafranov equation (2.46).

\subsubsection{Particle Conservation Equation}

Beginning with Eq. (2.2), the derivation of the flux averaged equations expressing the conservation of particles is obtained by integrating Eq. (2.2) over the volume interior to a surface $\rho=$ const

$$
\int \frac{\partial n_{j}}{\partial t} d \vec{x}+\int \nabla \cdot n_{j} \vec{u}_{j} d \vec{x}=\int S_{n j} d \vec{x}
$$

Note that in general $\rho$ can be an arbitrary surface label. Applying the divergence theorem on the second term of the left hand side of Eq. (2.51) it follows that 


$$
\int \frac{\partial n_{j}}{\partial t} d \vec{x}+\oint \frac{d S}{|\nabla \rho|} n_{j} \vec{u}_{j} \cdot \nabla \rho=\int S_{n j} d \vec{x}
$$

The above integrals are carried out over a region interior to the moving surface $\rho=$ const. Therefore, the first term in Eq. (2.52) takes the form[7]

$$
\left.\int \frac{\partial n_{j}}{\partial t}\right|_{\vec{x}} d \vec{x}=\left.\frac{\partial}{\partial t}\right|_{\rho} \int n_{j} d \vec{x}-\oint \frac{d S}{|\nabla \rho|} n_{j} \vec{u}_{\rho} \cdot \nabla \rho
$$

where, as indicated, the time derivative of $n_{j}$ is taken at constant $\vec{x}$, and the time derivative of $\int n_{j} d \vec{x}$ is taken at constant $\rho . \vec{u}_{\rho}$ is the velocity of the coordinate system characterized by $\rho$ with respect to the stationary coordinate system $\vec{x}$.

By substituting Eq. (2.53) into Eq. (2.52) it follows that

$$
\left.\frac{\partial}{\partial t}\right|_{\rho} \int n_{j} d \vec{x}+\oint n_{j}\left(\vec{u}_{j}-\vec{u}_{\rho}\right) \cdot \frac{\nabla \rho}{|\nabla \rho|} d S=\int S_{n j} d \vec{x}
$$

Finally by averaging over the poloidal angle $\theta$ Eq. (2.54) becomes

$$
\left.\frac{\partial}{\partial t}\right|_{\rho}\left(V^{\prime}\left\langle n_{j}\right\rangle\right)+\frac{\partial}{\partial \rho}\left(V^{\prime}\left\langle n_{j}\left(\vec{u}_{j}-\vec{u}_{\rho}\right) \cdot|\nabla \rho|\right\rangle\right)=V^{\prime}\left\langle S_{n j}\right\rangle
$$

By defining

$$
\Gamma_{j} \equiv\left\langle n_{j}\left(\vec{u}_{j}-\vec{u}_{\rho}\right) \cdot|\nabla \rho|\right\rangle
$$

to be the flux relative to the surfaces of constant $\rho, \mathrm{Eq}(2.55)$ becomes

$$
\frac{\partial}{\partial t}\left(V^{\prime}\left\langle n_{j}\right\rangle\right)+\frac{\partial}{\partial \rho}\left(V^{\prime} \Gamma_{j}\right)=V^{\prime}\left\langle S_{n j}\right\rangle
$$

The above equation describes the evolution of the density $n_{j}$ of species $j$ in the toroidal tokamak geometry. 


\subsubsection{Energy Conservation Equation}

The heat balance equation for the $\mathrm{j}^{\text {th }}$ species is given by

$$
\frac{3}{2} \frac{\partial\left(n_{j} T_{j}\right)}{\partial t}+\nabla \cdot\left[\vec{q}_{j}+\frac{5}{2} n_{j} T_{j} \vec{u}_{j}\right]=-\mathcal{P}_{j}: \nabla \vec{u}_{j}+\vec{u}_{j} \cdot \nabla p_{j}+Q_{j}+S_{E j}
$$

where $\mathcal{P}_{j}$, the anisotropic pressure tensor, is given by Eq. (2.17).

Proceeding in a manner similar to that used in the development of the mass conservation equation (section 2.5.1) the flux surface average of Eq. (2.58) is given by

$$
\begin{aligned}
\frac{3}{2} \frac{\partial}{\partial t}\left(V^{\prime}\left\langle n_{j} T_{j}\right\rangle\right) & =-\frac{\partial}{\partial \rho}\left[V^{\prime}\left(\left\langle\vec{q}_{j} \cdot \nabla \rho\right\rangle+\frac{5}{2}\left\langle n_{j} T_{j}\left(\vec{u}_{j}-\vec{u}_{\rho}\right) \cdot \nabla \rho\right\rangle\right)\right] \\
& -\frac{\partial}{\partial \rho} V^{\prime}\left\langle n_{j} T_{j} \vec{u}_{\rho} \cdot \nabla \rho\right\rangle \\
& -V^{\prime}\left\langle\mathcal{P}_{j}: \nabla \vec{u}_{j}\right\rangle+V^{\prime}\left\langle\vec{u}_{j} \cdot \nabla p_{j}\right\rangle \\
& +V^{\prime}\left\langle Q_{j}\right\rangle+V^{\prime}\left\langle S_{E j}\right\rangle
\end{aligned}
$$

During the time scale of cross field transport the densities and temperatures equilibrate along the magnetic field lines, and thus on any given flux surface the densities and temperatures are assumed constant

$$
\begin{aligned}
\left\langle n_{j}\right\rangle & \simeq n_{j} \\
\left\langle T_{j}\right\rangle & \simeq T_{j} \\
\left\langle n_{j} T_{j}\right\rangle & \simeq n_{j} T_{j}
\end{aligned}
$$

By using the above approximations the heat transport equation (2.59) becomes 


$$
\begin{aligned}
\frac{3}{2} \frac{\partial}{\partial t}\left(V^{\prime} n_{j} T_{j}\right) & =-\frac{\partial}{\partial \rho}\left[V^{\prime}\left(\left\langle\vec{q}_{j} \cdot \nabla \rho\right\rangle+\frac{5}{2} T_{j}\left\langle n_{j}\left(\vec{u}_{j}-\vec{u}_{\rho}\right) \cdot \nabla \rho\right\rangle\right)\right] \\
& -n_{j} T_{j} \frac{\partial V^{\prime}}{\partial t}-V^{\prime}\left\langle\vec{u}_{\rho} \cdot \nabla \rho\right\rangle \frac{\partial\left(n_{j} T_{j}\right)}{\partial \rho} \\
& -V^{\prime}\left\langle\mathcal{P}_{j}: \nabla \vec{u}_{j}\right\rangle+V^{\prime}\left\langle\vec{u}_{j} \cdot \nabla p_{j}\right\rangle \\
& +V^{\prime}\left\langle Q_{j}\right\rangle+V^{\prime}\left\langle S_{E j}\right\rangle
\end{aligned}
$$

Where the term $\partial V^{\prime} / \partial t$ is given by

$$
\frac{\partial V^{\prime}}{\partial t}=\frac{\partial}{\partial \rho}\left[V^{\prime}(\rho)\left\langle\vec{u}_{\rho} \cdot \nabla \rho\right\rangle\right]
$$

\subsection{Magnetic Diffusion Equation}

For a complete treatment of the plasma transport it is necessary to derive the equation for the evolution of the plasma poloidal flux. This equation must be solved consistently with the equations describing the evolution of the thermodynamic variables $n$, and $T$. The derivation is obtained by evoking Faraday's law, Ohm's law, and Ampere's law. The basic idea is to calculate the voltage induced due to the time change of the poloidal magnetic flux.

In general, the differential form of Faraday's law is

$$
\nabla \times \vec{E}=-\frac{\partial \vec{B}}{\partial t}
$$

where $\vec{E}$, and $\vec{B}$ are respectively the macroscopic electric and magnetic fields in the plasma. By integrating Eq. (2.65) around any moving contour of surface $S$ it is found that

$$
\frac{d}{d t} \int_{S} \vec{B} \cdot \widehat{e}_{n} d S=-\int_{S} \nabla \times \vec{E} \cdot \widehat{e}_{n} d S
$$


where $\widehat{e}_{n}$ is the unit vector normal to $d S$, and where $d / d t$ represents the convective derivative

$$
\frac{d}{d t}=\frac{\partial}{\partial t}+\vec{u}_{\rho} \cdot \nabla
$$

By using Stoke's theorem Eq. (2.66) becomes

$$
\frac{d}{d t} \int_{S} \vec{B} \cdot \widehat{e}_{n} d S=-\oint_{C} \vec{E} \cdot \overrightarrow{d l}
$$

where $C$ is a closed curve which bounds $S$, and $\overrightarrow{d l}$ is an infinitesimal displacement along $C$. In the tokamak geometry, by choosing the contour $C$ to be any axisymetric toroidal contour labeled by $\rho$ (see Fig. 2.2) Eq. (2.68) reduces to

$$
\frac{d}{d t} \int_{S} \vec{B} \cdot \widehat{e}_{n} d S=-2 \pi R E_{\phi}
$$

where $E_{\phi}$ is the toroidal component of the electric field, and $R$ is the major radius corresponding to the contour $C$. Since the magnetic field $B$ is related to the poloidal flux, $\psi_{P}$, via the equation

$$
\begin{aligned}
& \psi_{P}=\int_{S} \vec{B} \cdot \widehat{e}_{n} d S \\
& \psi_{P}=2 \pi \psi
\end{aligned}
$$

equation (2.69) becomes

$$
\frac{d \psi}{d t}=-R E_{\phi}
$$

The physical significance of Eq. (2.72) is that the toroidal voltage $V(\rho, t)=$ $2 \pi R E_{\phi}$ is induced due to the time change of the poloidal flux $\psi$. For future reference the electric field may thus be written as 


$$
\vec{E}=\frac{V(\rho, t)}{2 \pi R} \widehat{e}_{\phi}
$$

Now in order to estimate the voltage $V(\rho)$ it is required to evoke Ohm's law ( Eq. (2.23))

$$
\vec{E}=\eta \vec{J}-\vec{v} \times \vec{B}+\vec{E}_{\text {ext }}
$$

where $\vec{J}$ is the plasma current density, $\eta$ represents the plasma resistivity, $\vec{v}$ is the velocity of the plasma relative to the frame moving with the contours labeled with $\rho$, and where the term $\vec{E}_{\text {ext }}$ has been introduced to represent any externally applied electric fields. By taking the dot product of Eq. (2.74) with $\vec{B}$ it follows that

$$
\vec{E} \cdot \vec{B}=\eta \vec{J} \cdot \vec{B}+\vec{E}_{\text {ext }} \cdot \vec{B}
$$

Note that the unknown plasma relative velocity $\vec{v}$ has been eliminated from the above equation. By using Eq. (2.73) the flux surface average of Eq. (2.75) becomes

$$
\begin{aligned}
\langle\vec{E} \cdot \vec{B}\rangle & =\frac{V(\rho)}{2 \pi}\left\langle\frac{1}{R^{2}}\right\rangle F(\rho) \\
& =\eta\langle\vec{J} \cdot \vec{B}\rangle+\left\langle\vec{E}_{\text {ext }} \cdot \vec{B}\right\rangle
\end{aligned}
$$

where $F(\rho) \equiv R B_{\phi}$

The next step in the derivation requires the consideration of Ampere's law

$$
\nabla \times \vec{B}=\mu_{0} \vec{J}
$$

By using $\vec{B}=\vec{B}_{P}+B_{\phi} \widehat{e}_{\phi}$, where $B_{P}$, and $B_{\phi}$ are respectively the poloidal and toroidal components of the magnetic field, Eq. (2.78) becomes 


$$
\mu_{0} \vec{J}=\nabla F(\rho) \times \nabla \phi-\nabla \cdot\left(\frac{1}{R^{2}} \nabla \psi\right) R \widehat{e}_{\phi}
$$

By taking the dot product of Eq. (2.79) with $\vec{B}$, the following results:

$$
\mu_{0} \vec{J} \cdot \vec{B}=F^{\prime}(\rho) \frac{\partial \psi}{\partial \rho} \frac{|\nabla \rho|^{2}}{R^{2}}-\nabla \cdot\left(\frac{1}{R^{2}} \nabla \psi\right) F(\rho)
$$

The flux surface average of Eq. (2.80) gives

$$
\mu_{0}\langle\vec{J} \cdot \vec{B}\rangle=F^{\prime}(\rho) \frac{\partial \psi}{\partial \rho}\left\langle\frac{|\nabla \rho|^{2}}{R^{2}}\right\rangle-F(\rho) \frac{1}{V^{\prime}(\rho)} \frac{\partial}{\partial \rho}\left[V^{\prime}\left\langle\frac{|\nabla \rho|^{2}}{R^{2}}\right\rangle \frac{\partial \psi}{\partial \rho}\right]
$$

which is equivalent to

$$
\mu_{0}\langle\vec{J} \cdot \vec{B}\rangle=-\frac{F^{2}(\rho)}{V^{\prime}(\rho)} \frac{\partial}{\partial \rho}\left[\frac{V^{\prime}(\rho)}{F(\rho)}\left\langle\frac{|\nabla \rho|^{2}}{R^{2}}\right\rangle \frac{\partial \psi}{\partial \rho}\right]
$$

Finally, by combining Eqs. $(2.72,2.73,2.76,2.82)$ the magnetic diffusion equation becomes

$$
\frac{d \psi}{d t}=\frac{F(\rho)}{\left\langle 1 / R^{2}\right\rangle} \frac{\eta}{V^{\prime}(\rho)} \frac{\partial}{\partial \rho}\left[\frac{V^{\prime}(\rho)}{F(\rho)}\left\langle\frac{|\nabla \rho|^{2}}{R^{2}}\right\rangle \frac{\partial \psi}{\partial \rho}\right]+\frac{\left\langle\vec{E}_{\text {ext }} \cdot \vec{B}\right\rangle}{\left\langle 1 / R^{2}\right\rangle F(\rho)}
$$

In order to evolve the magnetic diffusion equation (2.83) it is necessary to determine the function $F(\rho)$. The determination of $F(\rho)$ is obtained by considering the force balance (Grad - Shafranov) equation (2.46). In particular $F(\rho)$ must satisfy the equation

$$
\frac{\partial \psi}{\partial \rho} \frac{1}{V^{\prime}(\rho)} \frac{\partial}{\partial \rho}\left[V^{\prime}(\rho)\left\langle\frac{|\nabla \rho|^{2}}{R^{2}}\right\rangle \frac{\partial \psi}{\partial \rho}\right]=\mu_{0} P^{\prime}(\rho)+\left\langle\frac{1}{R^{2}}\right\rangle F F^{\prime}
$$


For the solution of the above equation it is required that expressions for the plasma pressure profile $P$ and the plasma flux profile $\psi$ are provided. The pressure profile $P=n T$ is obtained from the density and temperature equations $(2.57,2.63)$, and the flux profile is obtained from Eq. (2.83).

The flux surface averaged plasma current density profile is obtained once the profiles of $P, \psi$, and $F$ have been determined, and is given by

$$
\left\langle J_{\phi}\right\rangle=\frac{1}{\partial \psi / \partial \rho}\left[\langle R\rangle P^{\prime}(\rho)+\left\langle\frac{1}{R}\right\rangle F F^{\prime}(\rho)\right]
$$

The plasma current enclosed by a flux surface labeled by $\rho$ is given by

$$
I_{P}(\rho)=-\frac{1}{\mu_{0}} V_{\rho}^{\prime} \frac{\partial \psi}{\partial \rho}\left\langle\frac{|\nabla \rho|^{2}}{R^{2}}\right\rangle
$$

\subsection{Complete $1 \frac{1}{2}-$ D Plasma Transport Model}

Having completed the development of the general flux surface averaged $\left(1 \frac{1}{2}-D\right)$ transport equations for mass, heat, and flux, the next step is to develop a working plasma transport model.

A future tokamak reactor will be fueled by deuterium and tritium and the resulting plasma will be composed of deuterium ions, tritium ions, fusion-product alpha particles, various impurity ions, as well as the accompanying electrons. In order to describe the behavior of such a plasma the evolution of the following quantities must be considered 
- Deuterium density

- Tritium density

- Alpha Particle density

- Impurity density

- Electron density

- Deuterium temperature

- Tritium temperature

- Alpha particle temperature

- Impurity temperature

- Electron temperature

- Magnetic flux $n_{d}$

$n_{t}$

$n_{\alpha}$

$n_{i m p}$

$n_{e}$

$T_{d}$

$T_{t}$

$T_{\alpha}$

$T_{i m p}$

$T_{e}$

$\psi$

\subsubsection{Transport Equations}

The evolution of the density, temperature, and flux is governed by equations Eqs. $(2.57,2.63,2.83)$ respectively. The equations describing the complete evolution of a deuterium tritium plasma thus become.

$$
\begin{aligned}
\frac{\partial}{\partial t}\left(V^{\prime} n_{d}\right) & =-\frac{\partial}{\partial \rho}\left(V^{\prime} \Gamma_{d}\right)+V^{\prime}\left\langle S_{n d}\right\rangle \\
\frac{\partial}{\partial t}\left(V^{\prime} n_{t}\right) & =-\frac{\partial}{\partial \rho}\left(V^{\prime} \Gamma_{t}\right)+V^{\prime}\left\langle S_{n t}\right\rangle \\
\frac{\partial}{\partial t}\left(V^{\prime} n_{\alpha}\right) & =-\frac{\partial}{\partial \rho}\left(V^{\prime} \Gamma_{\alpha}\right)+V^{\prime}\left\langle S_{n \alpha}\right\rangle \\
\frac{\partial}{\partial t}\left(V^{\prime} n_{i m p}\right) & =-\frac{\partial}{\partial \rho}\left(V^{\prime} \Gamma_{i m p}\right)+V^{\prime}\left\langle S_{n(i m p)}\right\rangle
\end{aligned}
$$




$$
\begin{aligned}
& \frac{\partial}{\partial t}\left(V^{\prime} n_{e}\right)=-\frac{\partial}{\partial \rho}\left(V^{\prime} \Gamma_{e}\right)+V^{\prime}\left\langle S_{n e}\right\rangle \\
& \frac{3}{2} \frac{\partial}{\partial t}\left(V^{\prime} n_{d} T_{d}\right)=-\frac{\partial}{\partial \rho}\left[V^{\prime}\left(\left\langle\vec{q}_{d} \cdot \nabla \rho\right\rangle+\frac{5}{2} T_{d}\left\langle n_{d}\left(\vec{u}_{d}-\vec{u}_{\rho}\right) \cdot \nabla \rho\right\rangle\right)\right] \\
& -n_{d} T_{d} \frac{\partial V^{\prime}}{\partial t}-V^{\prime}\left\langle\vec{u}_{\rho} \cdot \nabla \rho\right\rangle \frac{\partial}{\partial \rho}\left(n_{d} T_{d}\right) \\
& -V^{\prime}\left\langle\mathcal{P}_{d}: \nabla \vec{u}_{d}\right\rangle+V^{\prime}\left\langle\vec{u}_{d} \cdot \nabla p_{d}\right\rangle \\
& +V^{\prime}\left\langle Q_{d}\right\rangle+V^{\prime}\left\langle S_{E d}\right\rangle \\
& \frac{3}{2} \frac{\partial}{\partial t}\left(V^{\prime} n_{t} T_{t}\right)=-\frac{\partial}{\partial \rho}\left[V^{\prime}\left(\left\langle\vec{q}_{t} \cdot \nabla \rho\right\rangle+\frac{5}{2} T_{t}\left\langle n_{t}\left(\vec{u}_{t}-\vec{u}_{\rho}\right) \cdot \nabla \rho\right\rangle\right)\right] \\
& -n_{t} T_{t} \frac{\partial V^{\prime}}{\partial t}-V^{\prime}\left\langle\vec{u}_{\rho} \cdot \nabla \rho\right\rangle \frac{\partial}{\partial \rho}\left(n_{t} T_{t}\right) \\
& -V^{\prime}\left\langle\mathcal{P}_{t}: \nabla \vec{u}_{t}\right\rangle+V^{\prime}\left\langle\vec{u}_{t} \cdot \nabla p_{t}\right\rangle \\
& +V^{\prime}\left\langle Q_{t}\right\rangle+V^{\prime}\left\langle S_{E t}\right\rangle \\
& \frac{3}{2} \frac{\partial}{\partial t}\left(V^{\prime} n_{\alpha} T_{\alpha}\right)=-\frac{\partial}{\partial \rho}\left[V^{\prime}\left(\left\langle\vec{q}_{\alpha} \cdot \nabla \rho\right\rangle+\frac{5}{2} T_{\alpha}\left\langle n_{\alpha}\left(\vec{u}_{\alpha}-\vec{u}_{\rho}\right) \cdot \nabla \rho\right\rangle\right)\right] \\
& -n_{\alpha} T_{\alpha} \frac{\partial V^{\prime}}{\partial t}-V^{\prime}\left\langle\vec{u}_{\rho} \cdot \nabla \rho\right\rangle \frac{\partial}{\partial \rho}\left(n_{\alpha} T_{\alpha}\right) \\
& -V^{\prime}\left\langle\mathcal{P}_{\alpha}: \nabla \vec{u}_{\alpha}\right\rangle+V^{\prime}\left\langle\vec{u}_{\alpha} \cdot \nabla p_{\alpha}\right\rangle \\
& +V^{\prime}\left\langle Q_{\alpha}\right\rangle+V^{\prime}\left\langle S_{E \alpha}\right\rangle \\
& \frac{3}{2} \frac{\partial}{\partial t}\left(V^{\prime} n_{i m p} T_{i m p}\right)=-\frac{\partial}{\partial \rho}\left[V^{\prime}\left(\left\langle\vec{q}_{i m p} \cdot \nabla \rho\right\rangle+\frac{5}{2} T_{i m p}\left\langle n_{i m p}\left(\vec{u}_{i m p}-\vec{u}_{\rho}\right) \cdot \nabla \rho\right\rangle\right)\right] \\
& -n_{i m p} T_{i m p} \frac{\partial V^{\prime}}{\partial t}-V^{\prime}\left\langle\vec{u}_{\rho} \cdot \nabla \rho\right\rangle \frac{\partial}{\partial \rho}\left(n_{i m p} T_{i m p}\right) \\
& -V^{\prime}\left\langle\mathcal{P}_{i m p}: \nabla \vec{u}_{i m p}\right\rangle+V^{\prime}\left\langle\vec{u}_{i m p} \cdot \nabla p_{i m p}\right\rangle \\
& +V^{\prime}\left\langle Q_{i m p}\right\rangle+V^{\prime}\left\langle S_{E i m p}\right\rangle \\
& \frac{3}{2} \frac{\partial}{\partial t}\left(V^{\prime} n_{e} T_{e}\right)=-\frac{\partial}{\partial \rho}\left[V^{\prime}\left(\left\langle\vec{q}_{e} \cdot \nabla \rho\right\rangle+\frac{5}{2} T_{e}\left\langle n_{e}\left(\vec{u}_{e}-\vec{u}_{\rho}\right) \cdot \nabla \rho\right\rangle\right)\right] \\
& -n_{e} T_{e} \frac{\partial V^{\prime}}{\partial t}-V^{\prime}\left\langle\vec{u}_{\rho} \cdot \nabla \rho\right\rangle \frac{\partial}{\partial \rho}\left(n_{e} T_{e}\right)
\end{aligned}
$$




$$
\begin{aligned}
- & V^{\prime}\left\langle\mathcal{P}_{e}: \nabla \vec{u}_{e}\right\rangle+V^{\prime}\left\langle\vec{u}_{e} \cdot \nabla p_{e}\right\rangle \\
+ & V^{\prime}\left\langle Q_{e}\right\rangle+V^{\prime}\left\langle S_{E e}\right\rangle \\
\frac{\partial \psi}{\partial t}= & \frac{F(\rho)}{\left\langle 1 / R^{2}\right\rangle} \frac{\eta}{V^{\prime}(\rho)} \frac{\partial}{\partial \rho}\left[\frac{V^{\prime}(\rho)}{F(\rho)}\left\langle\frac{|\nabla \rho|^{2}}{R^{2}}\right\rangle \frac{\partial \psi}{\partial \rho}\right] \\
& +\frac{\left\langle\vec{E}_{\text {ext }} \cdot \vec{B}\right\rangle}{\left\langle 1 / R^{2}\right\rangle F(\rho)}-\left\langle\vec{u}_{\rho} \cdot \nabla \psi\right\rangle
\end{aligned}
$$

The above equations completely describe the evolution of the plasma thermodynamic variables $n_{d}, n_{t}, n_{\alpha}, n_{i m p}, n_{e}, T_{d}, T_{t}, T_{\alpha}, T_{i m p}$, and $T_{e}$, as well as the evolution of the poloidal flux $\psi$. The parameters $\Gamma_{d}, \Gamma_{t}$, $\Gamma_{\alpha}, \Gamma_{i m p}$, and $\Gamma_{e}$ represent the radial mass flow for the deuterium, tritium, alpha, impurity, and electron particles and can be written as

$$
\Gamma_{j}=-D_{j} \frac{\partial n_{j}}{\partial \rho}-v_{j} n_{j} \quad \text { where } \quad j=d, t, \alpha, i m p, e
$$

$\left\langle\vec{q}_{j} \cdot \nabla \rho\right\rangle$ represent the radial conductive heat flow of the $j^{\text {th }}$ species. $\left\langle Q_{j}\right\rangle$ gives the energy exchange between species $j$ and the rest of the plasma particles. $\left\langle S_{n j}\right\rangle$ corresponds to the mass sources and sinks of species $j$, and $\left\langle S_{E j}\right\rangle$ gives the sources and sinks for the energy of species $j$. The quantity $V^{\prime}$ is given by Eq. (2.40).

\subsubsection{Initial and Boundary Conditions}

The solution of Eqs. $(2.88-2.98)$ is obtained subject to the boundary conditions at the edge $(\rho=1)$ and on the axis $(\rho=0)$.

On the axis, the boundary conditions are

$$
\begin{aligned}
& \left.\frac{\partial n_{j}}{\partial \rho}\right|_{\rho=0}=0 \\
& \left.\frac{\partial T_{j}}{\partial \rho}\right|_{\rho=0}=0 \\
& \left.\frac{\partial \psi}{\partial \rho}\right|_{\rho=0}=0
\end{aligned}
$$


At the plasma surface $(\rho=1)$ the value or the gradient of each of the variables $n_{j}, T_{j}$, and $\psi$ is specified.

The initial conditions for the particle densities and temperatures may be given by any profile which satisfies the boundary conditions outlined above. The initial flux (poloidal magnetic field) profile can be specified by giving some form of the initial plasma current profile with the appropriate normalization in order to match the boundary condition for the poloidal flux.

\subsubsection{Simplified $1 \frac{1}{2}-$ D Plasma Transport Model}

By evoking standard plasma physics assumptions the number of thermodynamic variables to be evolved, and thus the number of equations to be solved, can be decreased. First, by assuming quasineutrality [8] the plasma electron density is given by

$$
n_{e} \equiv n_{d}+n_{t}+2 n_{\alpha}+\sum_{j} Z_{(i m p) j} n_{(i m p) j}
$$

where $Z_{(i m p) j}$ is the charge of the $j^{\text {th }}$ impurity ion with density $n_{(i m p) j}$. Therefore, the evolution of the electron density is governed by the behavior of the plasma ions. In the formulation that follows only one impurity species is considered. Further simplifications are obtained by assuming that all the plasma ions are in thermal equilibrium, i.e.

$$
T_{i} \equiv T_{d}=T_{t}=T_{\alpha}=T_{i m p}
$$

where $T_{i}$ will henceforth signify the plasma ion temperature.

By summing Eqs. $(2.93,2.94,2.95,2.96)$ and using Eqs. $(2.104,2.103)$ the system of flux averaged plasma transport equations becomes

$$
\frac{\partial}{\partial t}\left(V^{\prime} n_{d}\right)=-\frac{\partial}{\partial \rho}\left(V^{\prime} \Gamma_{d}\right)+V^{\prime}\left\langle S_{n d}\right\rangle
$$




$$
\begin{aligned}
& \frac{\partial}{\partial t}\left(V^{\prime} n_{t}\right)=-\frac{\partial}{\partial \rho}\left(V^{\prime} \Gamma_{t}\right)+V^{\prime}\left\langle S_{n t}\right\rangle \\
& \frac{\partial}{\partial t}\left(V^{\prime} n_{\alpha}\right)=-\frac{\partial}{\partial \rho}\left(V^{\prime} \Gamma_{\alpha}\right)+V^{\prime}\left\langle S_{n \alpha}\right\rangle \\
& \frac{\partial}{\partial t}\left(V^{\prime} n_{i m p}\right)=-\frac{\partial}{\partial \rho}\left(V^{\prime} \Gamma_{i m p}\right)+V^{\prime}\left\langle S_{n(i m p)}\right\rangle \\
& \frac{3}{2} \frac{\partial}{\partial t}\left(V^{\prime} n_{i} T_{i}\right)=-\frac{\partial}{\partial \rho}\left[V^{\prime}\left(\left\langle\vec{q}_{i} \cdot \nabla \rho\right\rangle+\frac{5}{2} T_{i} \Gamma_{i}\right)\right] \\
& -n_{i} T_{i} \frac{\partial V^{\prime}}{\partial t}-V^{\prime}\left\langle\vec{u}_{\rho} \cdot \nabla \rho\right\rangle \frac{\partial\left(n_{i} T_{i}\right)}{\partial \rho} \\
& \text { - } V^{\prime}\left\langle\mathcal{P}_{i}: \nabla \vec{u}_{i}\right\rangle+V^{\prime}\left\langle\vec{u}_{i} \cdot \nabla p_{i}\right\rangle \\
& +V^{\prime}\left\langle Q_{i}\right\rangle+V^{\prime}\left\langle S_{E i}\right\rangle \\
& \frac{3}{2} \frac{\partial}{\partial t}\left(V^{\prime} n_{e} T_{e}\right)=-\frac{\partial}{\partial \rho}\left[V^{\prime}\left(\left\langle\vec{q}_{\boldsymbol{e}} \cdot \nabla \rho\right\rangle+\frac{5}{2} T_{e} \Gamma_{e}\right)\right] \\
& \text { - } n_{e} T_{e} \frac{\partial V^{\prime}}{\partial t}-V^{\prime}\left\langle\vec{u}_{\rho} \cdot \nabla \rho\right\rangle \frac{\partial\left(n_{e} T_{e}\right)}{\partial \rho} \\
& -V^{\prime}\left\langle\mathcal{P}_{\boldsymbol{e}}: \nabla \vec{u}_{\boldsymbol{e}}\right\rangle+V^{\prime}\left\langle\vec{u}_{\boldsymbol{e}} \cdot \nabla p_{\boldsymbol{e}}\right\rangle \\
& -V^{\prime}\left\langle Q_{i}\right\rangle+V^{\prime}\left\langle S_{E e}\right\rangle \\
& \frac{\partial \psi}{\partial t}=\frac{F(\rho)}{\left\langle 1 / R^{2}\right\rangle} \frac{\eta}{V^{\prime}(\rho)} \frac{\partial}{\partial \rho}\left[\frac{V^{\prime}(\rho)}{F(\rho)}\left\langle\frac{|\nabla \rho|^{2}}{R^{2}}\right\rangle \frac{\partial \psi}{\partial \rho}\right] \\
& +\frac{\left\langle\vec{E}_{e x t} \cdot \vec{B}\right\rangle}{\left\langle 1 / R^{2}\right\rangle F(\rho)}-\left\langle\vec{u}_{\rho} \cdot \nabla \psi\right\rangle
\end{aligned}
$$

where the terms $\Gamma_{e}$ and $\Gamma_{i}$ are given by

$$
\begin{aligned}
\Gamma_{e} & =\left\langle n_{e}\left(\vec{u}_{e}-\vec{u}_{\rho}\right) \cdot \nabla \rho\right\rangle \\
\Gamma_{i} & =\left\langle n_{i}\left(\vec{u}_{i}-\vec{u}_{\rho}\right) \cdot \nabla \rho\right\rangle
\end{aligned}
$$

$\left\langle\vec{q}_{i} \cdot \nabla \rho\right\rangle$ corresponds to the total radial conductive heat flow of all the plasma ions, and $Q_{i}$ represents the heat exchange between the ions and the plasma electrons.[3] In general, the radial conductive heat flow is given by 


$$
\begin{aligned}
& \left\langle\vec{q}_{e} \cdot \nabla \rho\right\rangle=-\chi_{e} n_{e}|\nabla \rho|^{2} \frac{\partial T_{e}}{\partial \rho} \\
& \left\langle\vec{q}_{i} \cdot \nabla \rho\right\rangle=-\chi_{i} n_{i}|\nabla \rho|^{2} \frac{\partial T_{i}}{\partial \rho}
\end{aligned}
$$

where $\chi_{e}$, and $\chi_{i}$ are the electron and ion thermal diffusivities in units of [length ${ }^{2} /$ time].

The ion density, $n_{i}$, is given by

$$
n_{i}=n_{d}+n_{t}+n_{\alpha}+n_{i m p}
$$

The difference between the electron temperature, $T_{e}$, and the ion temperature, $T_{i}$, is ostensibly determined by the term $Q_{i}$, which gives a measure of the heat generated as a consequence of collisions. [3]

The solution of the above Eqs. $(2.105-2.110)$ requires the characterization of the term $\partial V^{\prime} / \partial t$, and the velocity $\vec{u}_{\rho}$ of the flux surfaces. However, by considering the magnitude of the various terms in Eqs. $(2.105-2.110)$ further simplifications can be made. The transport problem has three time scales of interest: The time scale of heat transport, the time scale of mass (particle) transport, and the time scale of flux transport. The flow of heat, mass and flux across the plasma with a characteristic length $a$ (for example $a$ can be the minor radius of a tokamak plasma) can be characterized with the the bulk velocities $v_{T}, v_{m}$, and $v_{\psi}$ respectively. These flow velocities follow the scalings

$$
\begin{aligned}
& v_{T} \approx \frac{\chi}{a} \\
& v_{m} \approx \frac{D}{a} \\
& v_{\psi} \approx \frac{\eta}{\mu_{0} a}
\end{aligned}
$$


where $\chi$ is some effective heat diffusivity, and $D$ is an effective particle diffusion coefficient. In general $\frac{v_{x}}{v_{m}} \sim 5$ and $v_{\psi} \ll v_{m}$. Therefore

$$
v_{T}>v_{m} \gg v_{\psi}
$$

and the terms $\frac{\partial V^{\prime}}{\partial t}, \vec{u}_{\rho} \cdot \nabla \rho$, and $\mathcal{P}: \nabla p$ are smaller than the $\partial T / \partial t$ and $\vec{q} \cdot \nabla \rho$ terms and may be neglected without loss of generality. It must be noted that the inclusion of the terms $\partial V^{\prime} / \partial t$, and $\vec{u}_{\rho} \cdot \nabla \rho$ is required if studies of startup phenomena are to be investigated. This simplified model should be used only in cases for which the flux surfaces have evolved sufficiently, as for example during the flat top portion of a tokamak plasma discharge. In terms of the numerical scheme used to solve the transport equations this simplification implies that the plasma equilibrium will be held fixed during a time step to the value obtained from the previous time step (i.e. the Grad - Shafranov equation will be solved only between time steps). The equations thus become

$$
\begin{aligned}
\frac{\partial n_{d}}{\partial t}= & -\frac{1}{V^{\prime}} \frac{\partial}{\partial \rho}\left(V^{\prime} \Gamma_{d}\right)+\left\langle S_{n d}\right\rangle \\
\frac{\partial n_{t}}{\partial t}= & -\frac{1}{V^{\prime}} \frac{\partial}{\partial \rho}\left(V^{\prime} \Gamma_{t}\right)+\left\langle S_{n t}\right\rangle \\
\frac{\partial n_{\alpha}}{\partial t}= & -\frac{1}{V^{\prime}} \frac{\partial}{\partial \rho}\left(V^{\prime} \Gamma_{\alpha}\right)+\left\langle S_{n \alpha}\right\rangle \\
\frac{\partial n_{i m p}}{\partial t}= & -\frac{1}{V^{\prime}} \frac{\partial}{\partial \rho}\left(V^{\prime} \Gamma_{i m p}\right)+\left\langle S_{n(i m p)}\right\rangle \\
\frac{3}{2} \frac{\partial}{\partial t}\left(n_{i} T_{i}\right)= & -\frac{1}{V^{\prime}} \frac{\partial}{\partial \rho}\left[V^{\prime}\left(-n_{i} \chi_{i} \frac{\partial T_{i}}{\partial \rho}+\frac{5}{2} T_{i} \Gamma_{i}\right)\right] \\
& +\left\langle Q_{i}\right\rangle+\left\langle S_{E i}\right\rangle \\
\frac{3}{2} \frac{\partial}{\partial t}\left(n_{e} T_{e}\right)= & -\frac{1}{V^{\prime}} \frac{\partial}{\partial \rho}\left[V^{\prime}\left(-n_{e} \chi_{e} \frac{\partial T_{e}}{\partial \rho}+\frac{5}{2} T_{e} \Gamma_{e}\right)\right] \\
& -\left\langle Q_{i}\right\rangle+\left\langle S_{E e}\right\rangle
\end{aligned}
$$




$$
\begin{aligned}
\frac{\partial \psi}{\partial t}= & \frac{F(\rho)}{\left\langle 1 / R^{2}\right\rangle} \frac{\eta}{V^{\prime}(\rho)} \frac{\partial}{\partial \rho}\left[\frac{V^{\prime}(\rho)}{F(\rho)}\left\langle\frac{|\nabla \rho|^{2}}{R^{2}}\right\rangle \frac{\partial \psi}{\partial \rho}\right] \\
& +\frac{\left\langle\vec{E}_{e x t} \cdot \vec{B}\right\rangle}{\left\langle 1 / R^{2}\right\rangle F(\rho)}
\end{aligned}
$$

\subsection{Sources and Sinks for Energy}

The sources and sinks for energy in Eqs. $(2.125,2.126)$ are related to the processes of fusion reactions, radiation, auxiliary heating, ohmic heating, and collisional energy exchange. In all the formulae that follow the use of MKS units is implicit with the temperature $T_{e}$ and $T_{i}$ given in $\mathrm{keV}$, and the density in units of $10^{20} / \mathrm{m}^{3}$.

\subsubsection{Collisional Energy Exchange}

The heat acquired by the ions in collisions with the electrons is given by

$$
Q_{i}=\frac{3}{2} \frac{n_{e}}{\tau_{e q}}\left(T_{e}-T_{i}\right)
$$

where $m_{e}$ is the electron mass, $m_{i}$ is the ion mass, and $1 / \tau_{e q}$ is the electron collision frequency with the background ions

$$
\tau_{e q}=0.099 \frac{T_{e}^{3 / 2}}{\ln \Lambda n_{i}}
$$

where $\ln \Lambda$ is the Coulomb logarithm [8]

$$
\ln \Lambda=38.7+\ln \left(\frac{T_{e}}{n_{e}^{1 / 2}}\right)
$$

Eq. (2.128) shows that for constant density and in the absence of other heating and cooling mechanisms the two temperatures $T_{e}$, and $T_{i}$ would gradually approach a common value. 


\subsubsection{Fusion Power}

In a deuterium $(D)$ tritium $(T)$ plasma the dominant thermonuclear reaction is

$$
D+T \longrightarrow H e^{4}(3.52 \mathrm{MeV})+n(14.1 \mathrm{MeV})
$$

The $14.1 \mathrm{MeV}$ neutron leaves the plasma and gives up its energy to the surrounding structure. The $3.52 \mathrm{MeV}$ alpha particles $\mathrm{He}^{4}$ are doubly charged ions and thus they are, in principle, confined by the toroidal magnetic field. As a result they transfer their energy to the plasma ions thereby providing a self heating mechanism for the plasma.

The total fusion power density produced by the deuterium tritium reaction is

$$
p_{f}=E_{f} n_{d} n_{t} \overline{\sigma v}
$$

where $n_{d}$ is the deuterium density, $n_{t}$ is the tritium density, and $E_{f}$ corresponds to $17.62 \mathrm{MeV} . \overline{\sigma v}$ represents the $D T$ fusion reactivity.

The source of energy to the plasma is related to the energy associated with the fusion alpha particles $\left(H e^{4}\right)$. The fusion alpha particles transfer their energy to the plasma electrons and ions via collisions. If the fraction of alpha particle energy deposited to the ions is $f_{i}$, then the contribution of fusion power to the electrons $p_{\alpha e}$, and to the ions $p_{\alpha i}$ is given by

$$
\begin{aligned}
& p_{\alpha e}=1.602 \times 10^{-13}\left(1-f_{i}\right) n_{d} n_{t} \overline{\sigma v} \quad W / \mathrm{m}^{3} \\
& p_{\alpha i}=1.602 \times 10^{-13} f_{i} n_{d} n_{t} \overline{\sigma v} \quad W / \mathrm{m}^{3}
\end{aligned}
$$

A calculation for the fraction $f_{i}$ is given by Sigmar and Joyce. [9] By fitting the results presented in [9] the formula

$$
f_{i}=1-\exp \left[-0.015 T_{e}\right] \quad\left(T_{e} \text { in } \mathrm{keV}\right)
$$

has an accuracy $\pm 5 \%$ in the range $5-50 \mathrm{keV}$. 


\subsubsection{Ohmic Heating}

In the tokamak configuration the current flow in the plasma provides an intrinsic heating mechanism. This resistive, or ohmic heating, of the plasma is given by

$$
p_{\Omega}=\vec{E} \cdot \vec{J}
$$

with $\vec{E}$ representing the applied electric field and $\vec{J}$ the plasma current. By using Ohm's law the ohmic power density becomes

$$
p_{\Omega}=C_{N C} \eta\left\langle J_{\phi}\right\rangle\left\langle J_{\phi}\right\rangle
$$

where the flux averaged toroidal plasma current density $\left\langle J_{\phi}\right\rangle$ is given by Eq. (2.85). $\eta$ is the Spitzer classical plasma resistivity given by

$$
\eta=1.65 \times 10^{-8} \ln \Lambda Z_{e f f} \frac{1}{T^{3 / 2}} \quad \Omega m
$$

The constant $C_{N C}$ represents the enhancement of classical resistivity due to Neo-Classical effects.[6] $Z_{\text {eff }}$, the effective charge of the plasma, is given by

$$
Z_{\text {eff }}=\frac{1}{n_{e}} \sum_{j} Z_{j}^{2} n_{j}
$$

where $Z_{j}$ is the charge of the $j^{\text {th }}$ ion with density $n_{j}$, and where $n_{e}$ corresponds to the electron density. Implicit in the above equation for $Z_{\text {eff }}$ is the assumption of quasineutrality Eq. (2.103).

\subsubsection{Radiation Losses}

Energy is lost from the plasma via Bremsstrahlung radiation, line radiation, and synchrontron radiation. In this model only the contribution from the Bremsstrahlung and synchrontron radiation is considered. 


\subsubsection{Bremsstrahlung radiation}

The power density lost due to Bremsstrahlung is given by [10]

$$
p_{b}=5.3 \times 10^{3} Z_{\text {eff }} n_{e}^{2} T_{e}^{1 / 2} \quad W / m^{3}
$$

\subsubsection{Synchrontron radiation}

Synchrontron radiation is emitted as a result of the gyration of electrons around the magnetic field lines. The radiation is emitted at frequencies which are integral multiples of the fundamental frequency $\nu_{f}[11]$

$$
\nu_{f}=2.8 \times 10^{10} B \quad \mathrm{~Hz}
$$

where $B$, the toroidal magnetic field, is given in Tesla.

The total synchrontron power emitted by the plasma is approximately given by [12]

$$
p_{s} \simeq 6.21 \times 10^{3} n_{e} T_{e} B^{2}\left(1+\frac{T_{e}}{146}+\cdots\right) \quad W / m^{3}
$$

\subsubsection{Auxiliary Power}

Various forms of auxiliary power can be employed in a fusion experiment. Each type of auxiliary power is characterized by a certain power deposition profile $\left(f_{a}(\rho)\right)$. By considering the total amount of auxiliary power $\left(P_{a}\right)$ to be deposited in the plasma and a certain normalized power deposition profile, which resembles the characteristic deposition profile of the type of auxiliary power under investigation, the total auxiliary power density is given in terms of the normalizing factor

$$
p_{a}=\frac{P_{a}}{\int_{V} f_{a}(\rho) d V}
$$

By assuming that a fraction $c_{e}$ of $p_{a}$ is transferred to the electrons then

$$
\begin{aligned}
& p_{a e}=c_{e} p_{a} f_{a}(\rho) \\
& p_{a i}=\left(1-c_{e}\right) p_{a} f_{a}(\rho)
\end{aligned}
$$


Table 2.1: Typical values for the heat exchange time for a deuterium plasma at a temperature of $10 \mathrm{keV}$.

\begin{tabular}{|c|c|}
\hline \hline Density $\left(10^{20} / \mathrm{m}^{3}\right)$ & $\tau_{\text {eq }}(\mathrm{sec})$. \\
\hline 1.0 & 0.184 \\
2.0 & 0.092 \\
3.0 & 0.061 \\
\hline
\end{tabular}

\subsection{Fast Equilibration Approximation}

The difference between the electron temperature, $T_{\boldsymbol{e}}$, and the ion temperature, $T_{i}$, is a function of the heat exchange time $\tau_{e q}$ given by Eq. (2.129). For high density Tokamaks such as CIT and Alcator the equilibration time is very short (see table 2.1 for typical values) and thus, to the zeroth order the electrons and ions can be assumed to be in thermal equilibrium.

It is thus possible to make the assumptions

$$
\begin{array}{ll}
T_{e} & =T+T_{1} \quad \text { Where } \frac{T_{1}}{T} \ll 1 \\
T_{i} & =T
\end{array}
$$

By adding Eqs. $(2.125,2.126)$, and neglecting the convective fluxes $\Gamma_{e}$ and $\Gamma_{i}$, it is found that

$$
\begin{aligned}
\frac{3}{2}\left[\frac{\partial}{\partial t}\left(n_{e} T_{e}\right)+\frac{\partial}{\partial t}\left(n_{i} T_{i}\right)\right]= & \frac{1}{V^{\prime}} \frac{\partial}{\partial \rho} V^{\prime}\left[n_{e} \chi_{e} \frac{\partial T_{e}}{\partial \rho}+n_{i} \chi_{i} \frac{\partial T_{i}}{\partial \rho}\right] \\
& +\left\langle S_{e}\right\rangle+\left\langle S_{i}\right\rangle
\end{aligned}
$$

Substituting Eqs. $(2.146,2.147)$ into Eq. (2.148) yields

$$
\begin{aligned}
\frac{3}{2}\left[\frac{\partial}{\partial t}\left(n_{\mathbf{e}}\left(T+T_{1}\right)\right)+\frac{\partial}{\partial t}\left(n_{i} T\right)\right]= & \frac{1}{V^{\prime}} \frac{\partial}{\partial \rho} V^{\prime}\left[n_{\mathbf{e}} \chi_{\mathbf{e}} \frac{\partial\left(T+T_{1}\right)}{\partial \rho}+n_{i} \chi_{i} \frac{\partial T}{\partial \rho}\right] \\
& +\left\langle S_{\boldsymbol{e}}\right\rangle+\left\langle S_{i}\right\rangle
\end{aligned}
$$


In the zeroth order the above equation reduces to

$$
\begin{aligned}
\frac{3}{2} \frac{\partial}{\partial t}\left(n_{e}+n_{i}\right) T= & \frac{1}{V^{\prime}} \frac{\partial}{\partial \rho} V^{\prime}\left(n_{e} \chi_{e}+n_{i} \chi_{i}\right) \frac{\partial T}{\partial \rho} \\
& +\left\langle S_{e}(T)\right\rangle+\left\langle S_{i}(T)\right\rangle
\end{aligned}
$$

By subtracting Eq. (2.126) from Eq. (2.125) and substituting Eqs. (2.146 - 2.147) the result is

$$
\begin{aligned}
\frac{3}{2}\left[\frac{\partial}{\partial t}\left(n_{e}\left(T+T_{1}\right)\right)-\frac{\partial}{\partial t}\left(n_{i} T\right)\right]= & \frac{1}{V^{\prime}} \frac{\partial}{\partial \rho} V^{\prime}\left[n_{e} \chi_{e} \frac{\partial\left(T+T_{1}\right)}{\partial \rho}-n_{i} \chi_{i} \frac{\partial T}{\partial \rho}\right] \\
& -3 \frac{n_{e}}{\tau_{e q}} T_{1}+\left\langle S_{e}\right\rangle-\left\langle S_{i}\right\rangle
\end{aligned}
$$

An approximate expression for $T_{1}$ is obtained by retaining the first order terms in Eq. (2.151). In particular

$$
\begin{aligned}
T_{1} \approx & \frac{1}{3} \frac{\tau_{e q}}{n_{e}}\left[\left\langle S_{E e}(T)\right\rangle-\left\langle S_{E i}(T)\right\rangle\right]-\frac{1}{2} \frac{\tau_{e q}}{n_{e}} \frac{\partial}{\partial t}\left(n_{e}-n_{i}\right) T \\
& -\frac{1}{3} \frac{\tau_{e q}}{n_{e}} \frac{1}{V^{\prime}} \frac{\partial}{\partial \rho} V^{\prime}\left[\left(n_{e} \chi_{e}-n_{i} \chi_{i}\right) \frac{\partial T}{\partial \rho}\right]
\end{aligned}
$$

In summary the equations representing the fast temperature equilibration approximation are given by

$$
\begin{aligned}
\frac{\partial n_{d}}{\partial t} & =-\frac{1}{V^{\prime}} \frac{\partial}{\partial \rho}\left(V^{\prime} \Gamma_{d}\right)+\left\langle S_{n d}\right\rangle \\
\frac{\partial n_{t}}{\partial t} & =-\frac{1}{V^{\prime}} \frac{\partial}{\partial \rho}\left(V^{\prime} \Gamma_{t}\right)+\left\langle S_{n t}\right\rangle \\
\frac{\partial n_{\alpha}}{\partial t} & =-\frac{1}{V^{\prime}} \frac{\partial}{\partial \rho}\left(V^{\prime} \Gamma_{\alpha}\right)+\left\langle S_{n \alpha}\right\rangle
\end{aligned}
$$




$$
\begin{aligned}
\frac{\partial n_{i m p}}{\partial t} & =-\frac{1}{V^{\prime}} \frac{\partial}{\partial \rho}\left(V^{\prime} \Gamma_{i m p}\right)+\left\langle S_{n(i m p)}\right\rangle \\
\frac{3}{2} \frac{\partial}{\partial t}\left(n_{e}+n_{i}\right) T & =\frac{1}{V^{\prime}} \frac{\partial}{\partial \rho} V^{\prime}\left(n_{e} \chi_{e}+n_{i} \chi_{i}\right) \frac{\partial T}{\partial \rho} \\
& +\left\langle S_{e}(T)\right\rangle+\left\langle S_{i}(T)\right\rangle \\
T_{e} & =T+T_{1} \\
\frac{\partial \psi}{\partial t} & =\frac{F(\rho)}{\left\langle 1 / R^{2}\right\rangle} \frac{\eta}{V^{\prime}(\rho)} \frac{\partial}{\partial \rho}\left[\frac{V^{\prime}(\rho)}{F(\rho)}\left\langle\frac{|\nabla \rho|^{2}}{R^{2}}\right\rangle \frac{\partial \psi}{\partial \rho}\right] \\
& +\frac{\left\langle\vec{E}_{\text {ext }} \cdot \vec{B}\right\rangle}{\left\langle 1 / R^{2}\right\rangle F(\rho)}
\end{aligned}
$$

\subsection{Ohmic Constraint}

In many situations it is possible to solve the $1 \frac{1}{2}-\mathrm{D}$ transport equations without explicitly evolving the magnetic flux. For example during the flat top it can be shown that the current density profile is related to the temperature profile via the so called ohmic constraint.

The derivation of the ohmic constraint follows from the assumption that the evolution of the poloidal flux has reached equilibrium. The equation for the evolution of flux (Eq. 2.72) may be written as

$$
\frac{\partial \psi}{\partial t}+R^{2} \vec{E} \cdot \nabla \phi=V(t)
$$

where $V(t)$ is the externally applied toroidal voltage which is only a function of time. Therefore, for $\partial \psi / \partial t=0$ and for $\vec{E} \cdot \nabla \phi \simeq \eta J_{\phi} / R$ the steady state relation becomes

$$
\eta R J_{\phi}=V(t)
$$

and by considering the form of the Spitzer resistivity for $\eta$ (Eq. 2.138) the above equation becomes 


$$
\frac{\eta_{0}}{T^{3 / 2}} R J_{\phi}=V(t)
$$

where $\eta_{0}$ is constant. Thus the left hand side of the above equation must be independent of space $(\rho)$ which implies that

$$
\left\langle R J_{\phi}\right\rangle \sim T^{3 / 2}
$$

Having obtained $\left\langle R J_{\phi}\right\rangle$, the shape of the flux surfaces is obtained from

$$
\mu_{0}\left\langle R J_{\phi}\right\rangle=\mu_{0}\left\langle R^{2}\right\rangle P^{\prime}+F F^{\prime}
$$

In summary the equations representing the fast temperature equilibration approximation and the ohmic constraint are given by

$$
\begin{aligned}
\frac{\partial n_{d}}{\partial t} & =-\frac{1}{V^{\prime}} \frac{\partial}{\partial \rho}\left(V^{\prime} \Gamma_{d}\right)+\left\langle S_{n d}\right\rangle \\
\frac{\partial n_{t}}{\partial t} & =-\frac{1}{V^{\prime}} \frac{\partial}{\partial \rho}\left(V^{\prime} \Gamma_{t}\right)+\left\langle S_{n t}\right\rangle \\
\frac{\partial n_{\alpha}}{\partial t} & =-\frac{1}{V^{\prime}} \frac{\partial}{\partial \rho}\left(V^{\prime} \Gamma_{\alpha}\right)+\left\langle S_{n \alpha}\right\rangle \\
\frac{\partial n_{i m p}}{\partial t} & =-\frac{1}{V^{\prime}} \frac{\partial}{\partial \rho}\left(V^{\prime} \Gamma_{i m p}\right)+\left\langle S_{n(i m p)}\right\rangle \\
\frac{3}{2 \frac{\partial}{\partial t}\left(n_{e}+n_{i}\right) T} & =\frac{1}{V^{\prime}} \frac{\partial}{\partial \rho} V^{\prime}\left(n_{e} \chi_{e}+n_{i} \chi_{i}\right) \frac{\partial T}{\partial \rho} \\
T_{e} & \left.=T+T_{e}(T)\right\rangle+\left\langle S_{i}(T)\right\rangle \\
\left\langle R J_{\phi}\right\rangle & \sim T^{3 / 2} \\
\mu_{0}\left\langle R J_{\phi}\right\rangle & =\mu_{0}\left\langle R^{2}\right\rangle P^{\prime}+F F^{\prime}
\end{aligned}
$$

where $T_{1}$ is given by Eq. (2.152). 


\subsection{Sources and Sinks for Mass}

The terms $S_{n d}, S_{n t}, S_{n \alpha}$, and $S_{n(i m p)}$ in Eqs. $(2.121-2.124)$ are related to fusion processes, volumetric effects, fueling, and atomic processes.

$$
\begin{aligned}
S_{n d} & =-n_{d} n_{t} \overline{\sigma v}-n_{d} \frac{1}{V} \frac{d V}{d t}+S_{d} \\
S_{n t} & =-n_{t} n_{t} \overline{\sigma v}-n_{t} \frac{1}{V} \frac{d V}{d t}+S_{t} \\
S_{n \alpha} & =n_{d} n_{t} \sigma v-n_{\alpha} \frac{1}{V} \frac{d V}{d t} \\
S_{n(i m p)} & =R+I+S_{i m p}
\end{aligned}
$$

where $S_{d},\left(S_{t}\right)$ is the volumetric deuterium, (tritium) source rate, and $V$ is the total plasma volume. $S_{i m p}$ represents the volumetric impurity source rate, and $R, I$ give the rates of recombination and ionization of the impurity species.

\subsection{Zero Dimensional Transport Equations}

In this section the general considerations for the derivation of the zero dimensional transport equations is presented. The derivation is given by considering a two species plasma (electrons and ions). The transport equations for such a plasma can be written in the simplified form

$$
\begin{aligned}
\frac{3}{2} \frac{\partial\left(n_{e} T_{e}\right)}{\partial t} & =\nabla_{\perp} \cdot\left(n_{e} \chi_{e \perp} \nabla_{\perp} T_{e}\right)+S_{E e} \\
\frac{\partial n_{e}}{\partial t} & =\nabla_{\perp} \cdot\left(D_{e \perp} \nabla_{\perp} n_{e}\right)+S_{n e} \\
\frac{3}{2} \frac{\partial\left(n_{i} T_{i}\right)}{\partial t} & =\nabla_{\perp} \cdot\left(n_{i} \chi_{i \perp} \nabla_{\perp} T_{i}\right)+S_{E i} \\
\frac{\partial n_{i}}{\partial t} & =\nabla_{\perp} \cdot\left(D_{i \perp} \nabla_{\perp} n_{i}\right)+S_{n i}
\end{aligned}
$$


where $Q_{\perp}$ denotes the component of $Q$ in the radial direction. $\chi_{e, i \perp}$, and $D_{e, i \perp}$ represent the thermal and particle diffusion coefficients for the electrons (e), and ions $(i) . S_{E e, i}$, and $S_{n e, i}$ represent the sources for energy and particles respectively. There is usually no explicit evolution equation for the flux in the zero dimensional model.

In principle, the solution of Eqs. $(2.177$ - 2.180) determines the time evolution of $T_{e}, T_{i}, n_{e}$, and $n_{i}$. Knowing the solution, physical phenomena such as the onset of thermal runaway, and the achievement of ignition can be predicted. However, the transport coefficients $\chi_{e}, \chi_{i}, D_{e}$, and $D_{i}$ are not well known either experimentally or theoretically. The source of the difficulty is a multitude of instabilities that occur over different portions the plasma cross section. The turbulent transport coefficients are often anomalously large, by factors of as much as two orders of magnitude. Because of the complex nature of turbulent transport, there is as yet no universally agreed upon transport coefficients.

The usual approach to circumvent this problem is to average the conservation equations, $(2.177-2.180)$, over the plasma volume. Then, by substituting experimentally measured profiles for the density and temperature, and by introducing empirically determined confinement time coefficients for the energy and particle losses Eqs. $(2.177-2.180)$ become.

$$
\begin{aligned}
& \frac{3}{2} \overline{n_{e} \frac{\partial T_{e}}{\partial t}}=-\frac{3}{2} \frac{\overline{n_{e} T_{e}}}{\tau_{E e}}+\overline{S_{E e}} \\
& \overline{\frac{\partial n_{e}}{\partial t}}=-\frac{\overline{n_{e}}}{\tau_{n e}}+\overline{S_{n e}} \\
& \frac{3}{2} \overline{n_{i} \frac{\partial T_{i}}{\partial t}}=-\frac{3}{2} \overline{\frac{n_{i} T_{i}}{\tau_{E i}}}+\overline{S_{E i}} \\
& \overline{\frac{\partial n_{i}}{\partial t}}=-\frac{\overline{n_{i}}}{\tau_{n i}}+\overline{S_{n i}}
\end{aligned}
$$

where $\bar{Q}$ denotes the volume average of $Q$, i.e. 


$$
\bar{Q} \equiv \frac{1}{V} \int Q d V
$$

The parameters $\tau_{E e}, \tau_{E i}, \tau_{n e}$, and $\tau_{n i}$ represent the global confinement time for the electron energy, the ion energy, the electron density, and the ion density respectively, and are defined by

$$
\begin{aligned}
\frac{1}{\tau_{E e}} \frac{3}{2} \int n_{e} T_{e} r d r & \equiv-\int \nabla_{\perp} \cdot\left(n_{e} \chi_{e \perp} \nabla_{\perp} T_{e}\right) r d r \\
\frac{1}{\tau_{n e}} \int n_{e} r d r & \equiv-\int \nabla_{\perp} \cdot\left(D_{n e} \nabla_{\perp} n_{e}\right) r d r \\
\frac{1}{\tau_{E i}} \frac{3}{2} \int n_{i} T_{i} r d r & \equiv-\int \nabla_{\perp} \cdot\left(n_{i} \chi_{i \perp} \nabla_{\perp} T_{i}\right) r d r \\
\frac{1}{\tau_{n i}} \int n_{i} r d r & \equiv-\int \nabla_{\perp} \cdot\left(D_{n i} \nabla_{\perp} n_{i}\right) r d r
\end{aligned}
$$

\subsubsection{Zero Dimensional Transport Model of a Ther- monuclear Plasma}

Next generation fusion reactors will certainly be fueled by deuterium and tritium. The resulting plasma will be composed of deuterium ions, tritium ions, fusion-product alpha particles, various impurity ions, as well as the accompanying electrons. By assuming that the density of the impurity ions is constant $\left(n_{i m p}=\right.$ const. $)$, the evolution of the deuterium density $\left(n_{d}\right)$, tritium density $\left(n_{t}\right)$, alpha particle density $\left(n_{\alpha}\right)$, deuterium temperature $\left(T_{d}\right)$, tritium temperature $\left(T_{t}\right)$, thermalized alpha particle temperature $\left(T_{\alpha}\right)$, and electron temperature $\left(T_{\boldsymbol{e}}\right)$ is given by,

$$
\begin{aligned}
\frac{\partial \bar{n}_{d}}{\partial t} & =-\frac{\bar{n}_{d}}{\tau_{n d}}+\bar{S}_{n d} \\
\frac{\partial \bar{n}_{t}}{\partial t} & =-\frac{\bar{n}_{t}}{\tau_{n t}}+\bar{S}_{n t}
\end{aligned}
$$




$$
\begin{aligned}
\frac{\partial \bar{n}_{\alpha}}{\partial t} & =-\frac{\bar{n}_{\alpha}}{\tau_{n \alpha}}+\bar{S}_{n \alpha} \\
\frac{3}{2} \frac{\partial\left(\overline{n_{d} T_{d}}\right)}{\partial t} & =-\frac{3}{2} \frac{\overline{n_{d} T_{d}}}{\tau_{E d}}+\bar{S}_{E d} \\
\frac{3}{2} \frac{\partial\left(\overline{n_{t} T_{t}}\right)}{\partial t} & =-\frac{3}{2} \frac{\overline{n_{t} T_{t}}}{\tau_{E t}}+S_{E t} \\
\frac{3}{2} \frac{\partial\left(\overline{n_{\alpha} T_{\alpha}}\right)}{\partial t} & =-\frac{3}{2} \frac{\overline{n_{\alpha} T_{\alpha}}}{\tau_{E \alpha}}+\bar{S}_{E \alpha} \\
\frac{3}{2} \frac{\partial\left(\overline{n_{e} T_{e}}\right)}{\partial t} & =-\frac{3}{2} \frac{\overline{n_{e} T_{e}}}{\tau_{E e}}+\bar{S}_{E e}
\end{aligned}
$$

By assuming thermal equilibration among the electrons and ions

$$
T \equiv T_{e}=T_{d}=T_{t}=T_{\alpha}
$$

as well as quasineutrality ( Eq. (2.103)), the summation of Eqs. (2.193 2.196) gives

$$
\frac{3}{2} \frac{\partial \overline{\left(n_{e}+n_{i}\right) T}}{\partial t}=-\frac{3}{2} \frac{\overline{\left(n_{e}+n_{i}\right) T}}{\tau_{E}}+\bar{S}_{E e}+\bar{S}_{E i}
$$

where $n_{i} \equiv n_{d}+n_{t}+n_{\alpha}+n_{i m p}$

The quantity $\tau_{E}$ represents the total energy confinement time, and it is determined empirically by measuring all other terms in Eq. (2.198) over a wide range of parameters in many different Tokamaks. In practice measurements for $\tau_{E}$ are obtained only during the flat top part (steady state) of the discharge. Expressions for $\tau_{E}$ are obtained as functions of the machine parameters and plasma conditions characterizing the various experiments (see section 2.12.5). Obviously, $\tau_{E}$ is a number for any given experiment, whereas $\chi$ is a function. It is therefore not possible to infer a unique $\chi$ once $\tau_{E}$ has been determined.

In summary, the equations describing the evolution of the plasma are, 


$$
\begin{aligned}
\frac{3}{2}\left(n_{e}+n_{i}\right) \frac{\partial T}{\partial t} & =-\frac{3}{2} \frac{\left(n_{e}+n_{i}\right) T}{\tau_{E}}+S_{E e}+S_{E i} \\
\frac{\partial n_{d}}{\partial t} & =-\frac{n_{d}}{\tau_{n d}}+S_{n d} \\
\frac{\partial n_{t}}{\partial t} & =-\frac{n_{t}}{\tau_{n t}}+S_{n t} \\
\frac{\partial n_{\alpha}}{\partial t} & =-\frac{n_{\alpha}}{\tau_{n \alpha}}+S_{n \alpha}
\end{aligned}
$$

The volume average of the terms in Eqs. $(2.199-2.202)$ is evaluated by assuming the plasma has an elliptic cross section with profiles given by

$$
\begin{aligned}
n_{j} & =n_{j 0}\left(1-\frac{x^{2}}{a^{2}}-\frac{y^{2}}{a^{2} \kappa^{2}}\right)^{\nu_{n}} \quad j=d, t, \alpha \\
T & =T_{0}\left(1-\frac{x^{2}}{a^{2}}-\frac{y^{2}}{a^{2} \kappa^{2}}\right)^{\nu_{T}}
\end{aligned}
$$

Experimental measurements indicate that $\nu_{n} \approx 1 / 2$ for gas puffing while $\nu_{n} \approx 1$ for pellet injection. The on axis values $T_{0}$ and $n_{0}$ are related to the averages $\bar{T}$ and $\bar{n}$ by

$$
\begin{aligned}
& n_{0}=\left(\nu_{n}+1\right) \bar{n} \\
& T_{0}=\left(\nu_{T}+1\right) \bar{T}
\end{aligned}
$$

In this analysis it is assumed that all the density profiles are described by the same profile parameter $\nu_{n}$.

\subsubsection{Volume Averaged Sources and Sinks for En- ergy.}

In general the terms $S_{E e}$, and $S_{E i}$ can be expressed in terms of the ohmic $\left(p_{\Omega}\right)$, alpha particle $\left(p_{\alpha}\right)$, auxiliary $\left(p_{a}\right)$, Bremsstrahlung $\left(p_{b}\right)$, and synchrontron $\left(p_{s}\right)$ power densities.

$$
S_{E \boldsymbol{e}}+S_{E i}=p_{\Omega}+p_{\alpha}+p_{a}-p_{b}-p_{s}
$$




\subsubsection{Ohmic Power Density $\left(p_{\Omega}\right)$}

The ohmic heating power density is given by

$$
p_{\Omega}=\eta J^{2}
$$

where $\eta$ is the plasma classical (Spitzer) resistivity given by Eq. (2.138).

The toroidal current density $J$ is related to the temperature by the steady state ohmic relation

$$
J=J_{0}\left(\frac{T}{T_{0}}\right)^{3 / 2}
$$

where $T_{0}$ is the temperature on axis, and where the current density on axis, $J_{0}$, is determined by the sawtooth condition $q_{0}=1$.

$$
J_{0}=\left(\frac{1+\kappa^{2}}{\kappa}\right)\left(\frac{B_{0}}{\mu_{0} R_{0} q_{0}}\right)
$$

In the above equation $B_{0}$ denotes the toroidal magnetic field on axis, $R_{0}$ is the major radius, $\kappa$ the plasma ellipticity, and $\mu_{0}$ is the permeability of free space. Eq. 2.209 is assumed valid for $p_{a}=0$ as well as $p_{a} \neq 0$ in order to avoid the unrealistically optimistic situation of simultaneously peaked temperature profiles and broad current profiles.

The profile parameter $\nu_{T}$ can now be related to the kink safety factor $q_{*}$ by the definition [4]

$$
q_{*} \equiv \frac{2 \pi a^{2} \kappa B_{0}}{\mu_{0} R_{0} I}
$$

where $a$ denoted the plasma minor radius at the mid plane. From Eqs. 2.209 and 2.210 and by using the relation $I=\int J d A$, where $I$ is the total plasma current, we obtain

$$
\frac{q_{*}}{q_{0}}=\frac{2 \kappa}{1+\kappa^{2}}\left(1+1.5 \nu_{T}\right)
$$


The physical significance of Eq. 2.212 is that as the total current increases the temperature profile becomes broader.

Finally, the volume averaged ohmic power density is given by

$$
p_{\Omega}=\frac{1.045 \times 10^{4} \ln \Lambda Z_{e f f}}{1+1.5 \nu_{T}}\left(\frac{1+\kappa^{2}}{\kappa}\right)^{2} \frac{B_{0}^{2}}{q_{0}^{2} R_{0}^{2} T_{0}^{3 / 2}} \quad W / m^{3}
$$

\subsubsection{Alpha Power Density $\left(p_{\alpha}\right)$}

The volume averaged alpha power density is given by

$$
p_{\alpha}=\frac{5.6 \times 10^{5}}{\nu_{T}} n_{d, 0} n_{t, 0} F_{\alpha}\left(T_{0}\right) \quad W / m^{3}
$$

where

$$
F_{\alpha}\left(T_{0}\right)=\frac{10^{22}}{T_{0}^{\gamma}} \int_{0}^{T_{0}} \overline{\sigma v}(\xi) \xi^{\gamma-1} d \xi
$$

with $\overline{\sigma v}$ given in $m^{3} / \sec$ and $\gamma \equiv\left(2 \nu_{n}+1\right) / \nu_{T}$.

\subsubsection{Auxiliary Power Density $\left(p_{a}\right)$}

The average auxiliary power heating density is defined as the total power absorbed by the plasma, $P_{a}$, divided by the plasma volume. Thus

$$
p_{a}=\frac{P_{a}}{2 \pi^{2} a^{2} R_{0} \kappa}=5.07 \times 10^{4}\left(\frac{P_{a}}{a^{2} R_{0} \kappa}\right) \quad W / m^{3}
$$

\subsubsection{Bremsstrahlung Radiation $\left(p_{b}\right)$}

The volume averaged radiative loss due to Bremsstrahlung is given by

$$
p_{b}=\frac{5.3 \times 10^{3} Z_{e f f}}{2 \nu_{n}+.5 \nu_{T}+1} n_{e, 0}^{2} T_{0}^{1 / 2} \quad W / m^{3}
$$




\subsubsection{Synchrontron Radiation $\left(p_{s}\right)$}

The volume averaged synchrontron radiation power density in $W / m^{3}$ is given by

$$
p_{s} \simeq 6.21 \times 10^{3} \frac{n_{e, 0} T_{e, 0}}{1+\nu_{n}+\nu_{T}} B^{2}\left(1+\frac{T_{e, 0}}{146\left(1+\nu_{n}+\nu_{T}\right)}+\cdots\right)
$$

\subsubsection{Conduction Losses}

The term first term on the right hand side of equation (2.199) gives a measure of the energy loss due to thermal conduction across the plasma.

$$
p_{l}=-\frac{3}{2} \frac{\left(n_{e}+n_{i}\right) T}{\tau_{E}}
$$

It is now important to emphasize that all the physics of energy transport across the plasma is incorporated in $\tau_{E}$, the energy confinement time. In general two types of confinement scalings are considered. The ohmic (NeoAlcator) [13] scaling $\tau_{N A}$, and the auxiliary scaling $\tau_{A U}$. A discussion of these scalings as well as several expressions for them will be given in section 2.12.5. Since the ohmic scaling represents an upper limit on confinement a combination of ohmic and auxiliary scalings of the form [14]

$$
\begin{aligned}
\frac{1}{\tau_{E}} & =\left(\frac{1}{\tau_{N A}^{2}}+\frac{1}{\tau_{A U}^{2}}\right)^{1 / 2} \\
& =\frac{1}{\tau_{N A}}\left(1+\Lambda^{2}\right)^{1 / 2}
\end{aligned}
$$

is used in order to limit $\tau_{E}$. The volume average of the thermal conduction losses is given by

$$
p_{l}=\frac{1.2 \times 10^{5}}{\nu_{n}+\nu_{T}+1}\left(\frac{n_{e, 0}+n_{i, 0}}{\tau_{N A}}\right) T_{0}\left(1+\Lambda^{2}\right)^{1 / 2} \quad W / m^{3}
$$


where $\Lambda \equiv \tau_{N A} / \tau_{A U}$, and $n_{e, 0}, n_{i, 0}$ are the peak values of the electron and ion densities.

Eqs. $(2.213,2.214,2.216$, and $2.217,2.218,2.221)$ are now substituted in Eq. 2.199 and the power balance becomes

$$
\begin{aligned}
K_{l}\left(n_{e}+n_{i}\right) \frac{\partial T}{\partial t}=\quad & K_{\Omega} \frac{B^{2}}{q_{0}^{2} R^{2} T^{3 / 2}}+K_{\alpha} n_{d} n_{t} F_{\alpha}(T)-K_{b} n_{e}^{2} T^{1 / 2} \\
& -K_{l} \frac{\left(1+\Lambda^{2}\right)^{1 / 2}}{\tau_{N A}}\left(n_{e}+n_{i}\right) T+K_{a} \frac{P_{a}}{R a^{2}} \\
& -K_{s} B^{2} n_{e} T\left(1+\frac{T}{146\left(1+\nu_{n}+\nu_{T}\right)}\right)
\end{aligned}
$$

where the zero subscript has been suppressed from $T_{0}, B_{0}, R_{0}, n_{e, 0}, n_{i, 0}$, $n_{d 0}, n_{t 0}$, and the parameters $K_{j}$ are given in Appendix A.

\subsubsection{0-D Density Equations}

By assuming profiles of the form given by Eqs. $(2.203,2.204)$ the volume average of Eqs. $(2.173-2.175)$ gives

$$
\begin{aligned}
S_{n d} & =-0.01 \frac{1+\nu_{n}}{\nu_{T}} n_{d 0} n_{t 0} F_{\alpha}-n_{d 0} \frac{1}{V} \frac{d V}{d t}+\left(1+\nu_{n}\right) S_{d} \\
S_{n t} & =-0.01 \frac{1+\nu_{n}}{\nu_{T}} n_{d 0} n_{t 0} F_{\alpha}-n_{t 0} \frac{1}{V} \frac{d V}{d t}+\left(1+\nu_{n}\right) S_{t} \\
S_{n \alpha} & =0.01 \frac{1+\nu_{n}}{\nu_{T}} n_{d 0} n_{t 0} F_{\alpha}-n_{\alpha 0} \frac{1}{V} \frac{d V}{d t}
\end{aligned}
$$

By substituting Eqs. $(2.223-2.225)$ into Eqs. $(2.200-2.202)$ the evolution of the plasma density is described by the equations

$$
\frac{\partial n_{d}}{\partial t}=-\frac{n_{d}}{\tau_{d}}+\left(1+\nu_{n}\right) S_{d}-0.01 \frac{1+\nu_{n}}{\nu_{T}} n_{d} n_{t} F_{\alpha}(T)-n_{d} \frac{1}{V} \frac{d V}{d t}
$$




$$
\begin{aligned}
\frac{\partial n_{t}}{\partial t} & =-\frac{n_{t}}{\tau_{t}}+\left(1+\nu_{n}\right) S_{t}-0.01 \frac{1+\nu_{n}}{\nu_{T}} n_{d} n_{t} F_{\alpha}(T)-n_{t} \frac{1}{V} \frac{d V}{d t} \\
\frac{\partial n_{\alpha}}{\partial t} & =-\frac{n_{\alpha}}{\tau_{d}}+0.01 \frac{1+\nu_{n}}{\nu_{T}} n_{d} n_{t} F_{\alpha}(T)-n_{\alpha} \frac{1}{V} \frac{d V}{d t}
\end{aligned}
$$

where the zero subscript has been suppressed from $n_{d 0}, n_{t 0}$, and $n_{\alpha 0}$. The function $F_{\alpha}(T)$ is given by Eq. (2.215).

\subsubsection{Complete 0-D Transport Model}

The complete set of equations describing the evolution of the plasma temperature and density is

$$
\begin{aligned}
K_{l}\left(n_{e}+n_{i}\right) \dot{T}= & K_{\Omega} \frac{B^{2}}{q_{0}^{2} R^{2} T^{3 / 2}}+K_{\alpha} n_{d} n_{t} F_{\alpha}(T)-K_{b} n_{e}^{2} T^{1 / 2} \\
& -K_{l} \frac{\left(1+\Lambda^{2}\right)^{1 / 2}}{\tau_{N A}}\left(n_{e}+n_{i}\right) T+K_{a} \frac{P_{a}}{R a^{2}} \\
& -K_{s} B^{2} n_{e} T\left(1+\frac{T}{146\left(1+\nu_{n}+\nu_{T}\right)}\right) \\
\frac{\partial n_{d}}{\partial t}= & -\frac{n_{d}}{\tau_{d}}+\left(1+\nu_{n}\right) S_{d}-0.01 \frac{1+\nu_{n}}{\nu_{T}} n_{d} n_{t} F_{\alpha}(T)(2.230) \\
\frac{\partial n_{t}}{\partial t}= & -\frac{n_{t}}{\tau_{t}}+\left(1+\nu_{n}\right) S_{t}-0.01 \frac{1+\nu_{n}}{\nu_{T}} n_{d} n_{t} F_{\alpha}(T)(2.231) \\
\frac{\partial n_{\alpha}}{\partial t}= & -\frac{n_{\alpha}}{\tau_{d}}+0.01 \frac{1}{\nu_{T}} \frac{1}{V} \frac{d V}{d t} n_{d} n_{t} F_{\alpha}(T)-n_{\alpha} \frac{1}{V} \frac{d V}{d t} \\
n_{e} \equiv & n_{d}+n_{t}+2 n_{\alpha}+Z_{i m p} n_{i m p} \\
n_{i} \equiv & n_{d}+n_{t}+n_{\alpha}+n_{i m p}
\end{aligned}
$$




\subsubsection{Energy and Particle Confinement Scalings}

In order to predict phenomena such as the achievement of ignition or the onset of thermal instabilities the solution of Eqs. (2.229 - 2.232) must be obtained. The first step towards solving Eqs. $(2.229-2.232)$ is the characterization of the global confinement times: $\tau_{E}, \tau_{n d}, \tau_{n t}$, and $\tau_{n \alpha}$.

\subsubsection{Energy Confinement Time}

Through the years measurements of plasma properties, such as temperature, have been obtained as functions of toroidal field, plasma current, confinement geometry, and auxiliary power (the control "knobs"). Tokamak devices such as the Alcator $\mathrm{C}$ at MIT have operated with density ranging from $10^{19} \mathrm{~m}^{-3}$ to $10^{21} \mathrm{~m}^{-3}$, and with magnetic field as high as $12 \mathrm{~T}$. Other machines such as the TFTR tokamak at Princeton have operated with as much as $35 \mathrm{MW}$ of auxiliary power supplied to the plasma, resulting in a temperature up to $32 \mathrm{keV}$. Furthermore, DIIID at General Atomic in San Diego has achieved $\beta$ (plasma pressure / magnetic pressure) values of up to $8 \%$, and other machines such as the Joint European Torus (JET) operate with large plasmas (1.25 m minor radius) and high current (up to $7 \mathrm{MA}$ ).

The results from these experiments have generated a large data base in which the plasma conditions are given as functions of the various control knobs. A value for the energy confinement time $\tau_{E}$ can be obtained from Eq. (2.229) once the rest of the terms are known. By using the complete data base it is possible to obtain empirical scalings for the energy confinement time $\tau_{E}$ by regression analysis of the data. In general not the whole data base is used when a fit for $\tau_{E}$ is desired. This is because different modes of operation, such as the type of heating used, seem to imply different empirical forms for $\tau_{E}$. For example, when ohmic power is the dominant heating mechanism, a reasonably reliable and relatively optimistic scaling for $\tau_{E}$ is the so called "Neo-Alcator" scaling. However, in the important regime of auxiliary power dominated operation, several different, more pessimistic, but not dissimilar empirical scalings for $\tau_{E}$ have been derived. These types of confinement scalings are, in this thesis, labeled as the "Auxiliary Scalings". 


\subsubsection{Ohmic Scalings. Neo-Alcator}

Ohmic confinement, and thus the use of Neo-Alcator scaling for $\tau_{E}$, is obtained when ohmic heating represents the primary plasma heating mechanism. The Neo-Alcator scaling is given by [13]

$$
\tau_{N A}=0.2 \bar{n}_{e} a R_{0}^{2} \kappa^{1 / 2} \quad \text { sec. }
$$

where $\bar{n}_{e}$ is the line averaged electron density, $\kappa$ is the plasma ellipticity, and $a$, and $R_{0}$ correspond to the minor and major radius of the plasma. If the solution of Eqs. $(2.229-2.232)$ is desired under the assumption of Neo-Alcator scaling, then the parameter $\Lambda$ in Eq. (2.229) must be set equal to zero.

\subsubsection{Auxiliary Scalings}

One of the first energy confinement scaling for auxiliary heated tokamaks was was put forward by Goldston in 1982.[14] Since then a large number of scalings has been developed modeling this regime of operation. The main feature of these scalings is that confinement in auxiliary heated tokamaks degrades with power. This implies that the total energy confinement time $\tau_{E}$ characterizing the plasma decreases as the auxiliary power supplied to the plasma increases.

The Goldston scaling for the energy confinement time in auxiliary heated tokamaks is given by

$$
\tau_{G L}=0.037 M \frac{I_{p} \kappa^{.5} R^{1.75} A^{.5}}{P^{.5} a^{.37}} \quad s e c
$$

where the plasma current $I_{p}$ is given in MA, M is a multiplicative factor whose significance will be discussed shortly, and $A=5 / 3$ represents the normalized mass ratio for $\mathrm{D}-\mathrm{T}$ reactions. The term $P$ appearing in the denominator of Eq. (2.236) is given in MW and it represents the total amount of auxiliary power supplied to the plasma plus the total amount of 
alpha power produced by the fusion process. It is thus assumed that the alpha power will degrade confinement in future tokamaks in the same way that auxiliary power has been observed to degrade confinement in present fusion experiments. Under the assumption of Goldston scaling solutions of Eq. (2.229) are obtained with $\Lambda \equiv \Lambda_{G L}$, where

$$
\begin{aligned}
\Lambda_{G L} & =\frac{\tau_{N A}}{\tau_{G L}} \\
& =K_{G L} \frac{n_{e} R}{B} \Gamma^{1 / 2}
\end{aligned}
$$

where $\Gamma$, and $K_{G L}$ are given by

$$
\begin{aligned}
\Gamma & =C_{1} \frac{R P_{a}}{a}+C_{2}\left(\frac{n_{e} R}{B}\right)^{2} B^{2} a F_{\alpha}(T) \\
K_{G L} & =\frac{3.71}{M f} \frac{q_{*}}{R^{25} a^{\cdot 13} \kappa^{.75}}
\end{aligned}
$$

$F_{\alpha}(T)$ is given by Eq. 2.215 and $C_{1}, C_{2}$, and $f$ are given in Appendix A.

During the last five years various modes of enhanced confinement have been observed in tokamaks. These modes have been obtained through profile control, divertor operation, and edge preparation. It is observed that these enhancement confinement modes show many of the same scaling characteristics as the original scalings. Therefore, it is common to characterize this enhanced confinement by the multiplicative factor $M$. These enhanced confinement modes are referred to as the "H-mode" (for high confinement mode) as compared to the "L-mode" (for low confinement). Usually Hmode confinement is modeled by letting $M$ to be in the range $1.5-2.5$.

Similar expressions for $\Lambda$ can be obtained for other scalings such as Kaye-Goldston [15]

$$
\tau_{K G}=0.055 M \frac{I_{p}^{1.24} n^{.26} \kappa^{.28} R^{1.65} A^{.5}}{B^{0.09} P^{.5} a^{.49}}
$$




\subsubsection{Particle Confinement Time}

Contrary to the energy confinement time, $\tau_{E}$, for which a large number of empirical scalings exist, expressions for the global particle confinement time $\tau_{P}$ have not been developed. However, from measurements of the thermal diffusivity $\chi$, and the particle diffusivity $D$ in the JET $[16,17,18]$ and other tokamaks $[19]$ it appears that the central values of $\chi$ and $D$ are in the ratio

$$
\frac{\chi}{D}= \begin{cases}\frac{2.9 \pm 0.4}{0.4 \pm 0.1} & \text { For Ohmic Heating } \\ \frac{3.0 \pm 0.5}{0.4 \pm 0.2} & \text { For Auxiliary Heating }\end{cases}
$$

By considering the ratios of $\chi / D$ given above, and by using Eqs. $(2.186$ - 2.189) it is common to assume that the global particle confinement time $\tau_{P}$ is $\sim 3-5$ times the energy confinement time $\tau_{E}$. Therefore, in the analysis that follows the particle confinement time has the form

$$
\tau_{P}=C \tau_{E}
$$

where $C$ is a constant number between three and five.

\subsection{Conclusions}

The transport equations derived in this chapter will be solved in subsequent chapters in specific tokamak geometries. First, by solving the zero dimensional equations the concepts of Ignition and Burn Control are investigated. Next, by employing variational techniques, the flux surface averaged $\left(1 \frac{1}{2}-D\right)$ plasma transport equations are solved and the results are compared to those obtained from the zero dimensional transport model. 


\section{Chapter 3}

\section{Design Optimization of Ignited Tokamaks}

The zero dimensional power balance Eq. (2.229)can be written in the compact form.

$$
\tau_{E} \frac{d T}{d t}=-T+W\left[\frac{C_{\Omega}}{N T^{3 / 2}}+N\left(C_{\alpha} F-C_{b} T^{1 / 2}\right)\right]+C_{a} \frac{W \Pi}{N}
$$

Where, $T(t)$ is the peak temperature, $W \equiv B \tau_{E} / R$ is a basic parameter measuring machine performance, $N \equiv n R / B$ is a normalized form of the density, and $\Pi \equiv P_{a} R / a^{2} B^{2}$ is a normalized form of the total auxiliary power $P_{a}=\left\langle p_{a}\right\rangle V_{p}$ where $V_{p}$ is the plasma volume. The function $F(T)$ is given by Eq. (2.215), and the coefficients $C_{j}$ are given in Appendix A. In these expressions and all that follow, the units are $T(\mathrm{keV}), n\left(10^{20} \mathrm{~m}^{-3}\right)$, $B(\mathrm{~T}), a(\mathrm{~m}), R(\mathrm{~m}), \tau_{E}(\mathrm{sec})$, and $P_{a}(\mathrm{MW})$. In summary the quantities $W, N, \Pi$ are given by

$$
\begin{aligned}
W & =\frac{B \tau_{E}}{R} \\
N & =\frac{n R}{B} \\
\Pi & =\frac{P_{a} R}{a^{2} B^{2}}
\end{aligned}
$$


Equation (3.1) is the power balance relation describing the time evolution of $T$. There are many ways to rewrite Eq. (3.1) by defining a different set of parameters. The choices made here for $W$ and $N$ are crucial for a proper understanding of ignition. The critical issue is that $n$ and $\tau_{E}$ are not interchangeable parameters in the design of an ignition experiment. In a given device, $n \sim N$ can be easily varied over a reasonably wide range of parameters without affecting the cost. $N$ is constrained only by a practical experimental operating limit, ${ }^{1}$ known as the Murakami [22] limit given by

$$
N \leq N_{m}=1.4
$$

In contrast, raising $\tau_{E} \sim W$ invariably requires an increase in either field, current, or machine size and, hence, cost. Consequently, $W=B \tau_{E} / R$ gives a measure of machine performance and cost. It is more reliable than such traditional parameters as $n \tau_{E}$ or $n \tau_{E} T$ which do not distinguish between high n-low $\tau_{E}$ (low cost) or low n-high $\tau_{E}$ (high cost).

In general the energy confinement time $\tau_{E}$ can be written as

$$
\tau_{E}=\mathcal{G}(a, R, \kappa, \cdots) \mathcal{F}(N, T, \Pi)
$$

where $\mathcal{G}$ is a function of geometry $(a, R, \kappa, \cdots)$, and $\mathcal{F}$ is a function of temperature, density, and auxiliary power. Therefore, the paremeter $W$ may be written as

$$
\begin{aligned}
W & =\widehat{W} \mathcal{F}(N, T, \Pi) \\
\widehat{W} & =\frac{B}{R} \boldsymbol{g}(a, R, \kappa, \cdots)
\end{aligned}
$$

In this general case $\widehat{W}$ serves as a performance parameter. In particular the following cases are investigated.

\footnotetext{
${ }^{1}$ There is a second operational limit due to MHD instabilities known as the Troyon $[20,21]$ limit, $N<N_{\beta}=1.12 I R / a T$. In the analysis, the Murakami limit is assumed more severe. The $\beta$ limit is tested a posteori, once the final geometry is decided.
} 
-Generic. $\quad \mathcal{F}(N, T, \Pi)=1$

-Ohmic. $\quad \mathcal{F}(N, T, \Pi)=N$

-Auxiliary1. $\quad \mathcal{F}(N, T, \Pi)=1 / T^{0.5}$

-Auxiliary2. $\mathcal{F}(N, T, \Pi)=N / T$

\subsection{Ignition Definition}

The natural definition of ignition follows from an examination of the $\dot{T}$ vs $T$ diagram. Depending upon the size of $W$ there are three qualitatively different regimes to distinguish: ohmic ignition, auxiliary power ignition, and non-ignited operation.

Figure 3.1 illustrates the most desirable regime of operation. Observe that for $P_{a}=0$ there are three steady state equilibrium solutions corresponding to $\dot{T}=0$. The left point, denoted by $T_{\Omega}$, represents the temperature achieved in an ohmic discharge $\left\langle p_{\Omega}\right\rangle \approx\left\langle p_{l}\right\rangle$ and is dominated by $1 / T^{3 / 2}$ dependance of the ohmic power term. It typically occurs at $T_{\Omega} \sim 2-3 \mathrm{keV}$. The second equilibrium point $T_{B}$ is dominated by alpha particle heating and usually occurs at $T_{B} \sim 12-20 \mathrm{keV}$. Since the optimum temperature for fusion power production, at fixed $\beta$, occurs at $T \sim 15 \mathrm{keV}$ this is ultimately the desired operating temperature. The third equilibrium point, $T_{R}$, represents the final stable temperature. It typically occurs at $T_{R} \sim 50 \mathrm{keV}$ as a consequence of the high temperature decay of the $\overline{\sigma v}$ curve. This point, even though stable, is uninteresting because the plasma $\beta$ is far above the MHD stability limit and corresponds to operation far from at the optimum temperature for fusion power production.

Unfortunately, it is not possible to gain access to the high temperature equilibrium point $T_{B}$ through the normal evolution of the ohmic discharge. The reason is as follows. At the initiation of the discharge the temperature 


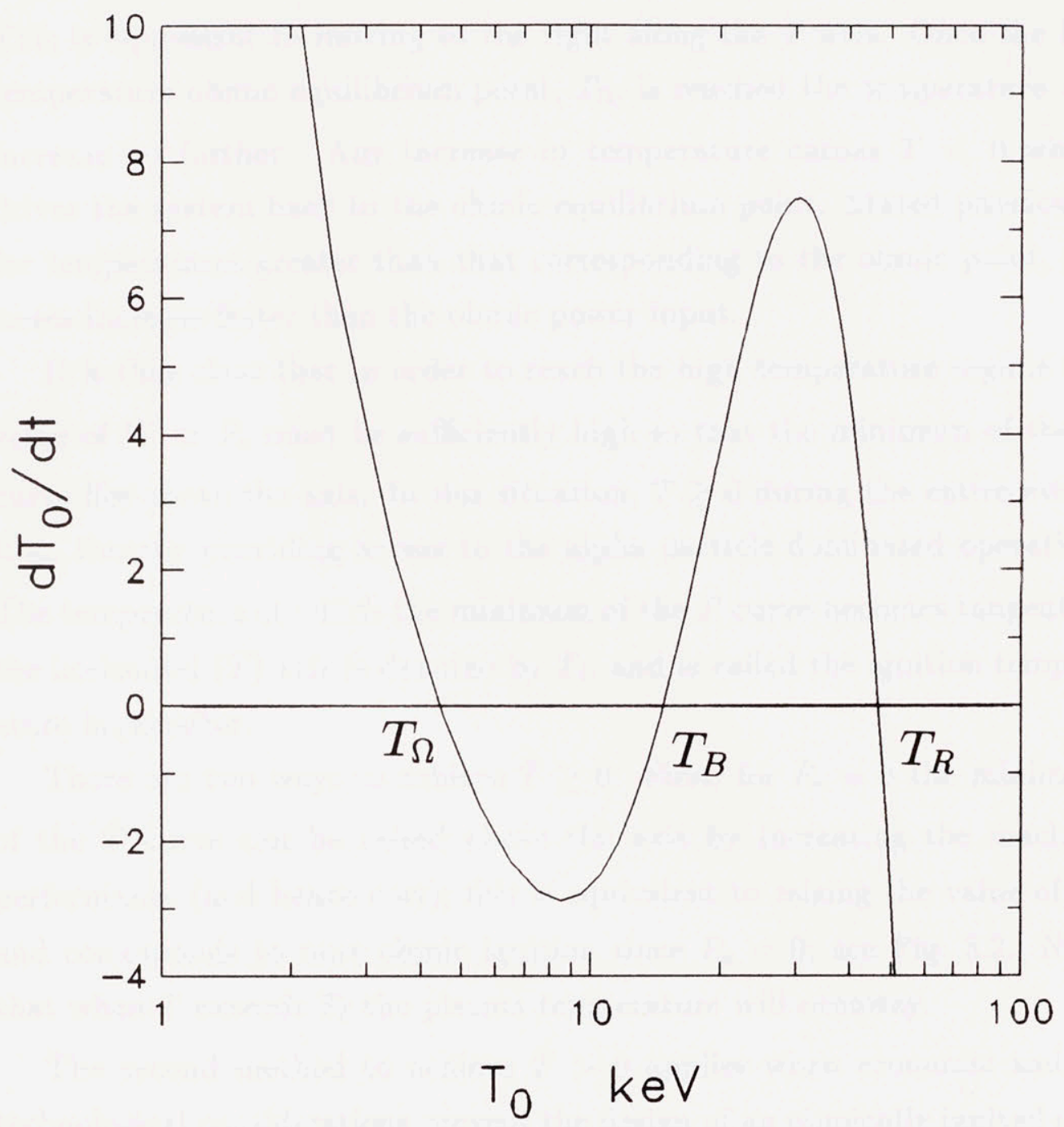

Figure 3.1: Illustrative $\dot{T}$ vs $T$ plot with the three equilibrium points $T_{\Omega}$, $T_{B}$, and $T_{R}$ 
is very low and ohmic heating is the dominant mechanism in the power balance relation. As time increases the temperature increases since $\dot{T}>0$. This is equivalent to moving to the right along the $T$ axis. Once the low temperature ohmic equilibrium point, $T_{\Omega}$, is reached the temperature can increase no further. Any increase in temperature causes $\dot{T}<0$ which drives the system back to the ohmic equilibrium point. Stated physically, for temperatures greater than that corresponding to the ohmic point, the losses increase faster than the ohmic power input.

It is thus clear that in order to reach the high temperature regime the value of $W$ or $P_{a}$ must be sufficiently high so that the minimum of the $\dot{T}$ curve lies above the axis. In this situation, $\dot{T} \geq 0$ during the entire evolution, thereby providing access to the alpha particle dominated operation. The temperature at which the minimum of the $\dot{T}$ curve becomes tangent to the horizontal $(T)$ axis is denoted by $T_{I}$, and is called the ignition temperature hereinafter.

There are two ways to achieve $\dot{T} \geq 0$. First, for $P_{a}=0$ the minimum of the $\dot{T}$ curve can be raised above the axis by increasing the machine performance (and hence cost); this is equivalent to raising the value of $W$ and corresponds to pure ohmic ignition since $P_{a}=0$; see Fig. 3.2. Note that when $T$ exceeds $T_{I}$ the plasma temperature will runaway.

The second method to achieve $\dot{T}>0$ applies when economic and/or technological considerations prevent the design of an ohmically ignited machine. In this case the minimum of the $\dot{T}$ curve can be made positive if sufficient auxiliary power is supplied to the plasma. This type of ignition is denoted as ignition with auxiliary power; see Fig. 3.3. Once $T_{I}$ is exceeded, the auxiliary power can be gradually decreased shifting operation to $T=T_{B}$ on the $P_{a}=0$ curve. Note that at $T=T_{B}$ the system is susceptible to a thermal runaway since $\dot{T}\left(T_{B}\right)>0$. This is the problem of burn control and is discused in chapter 4 


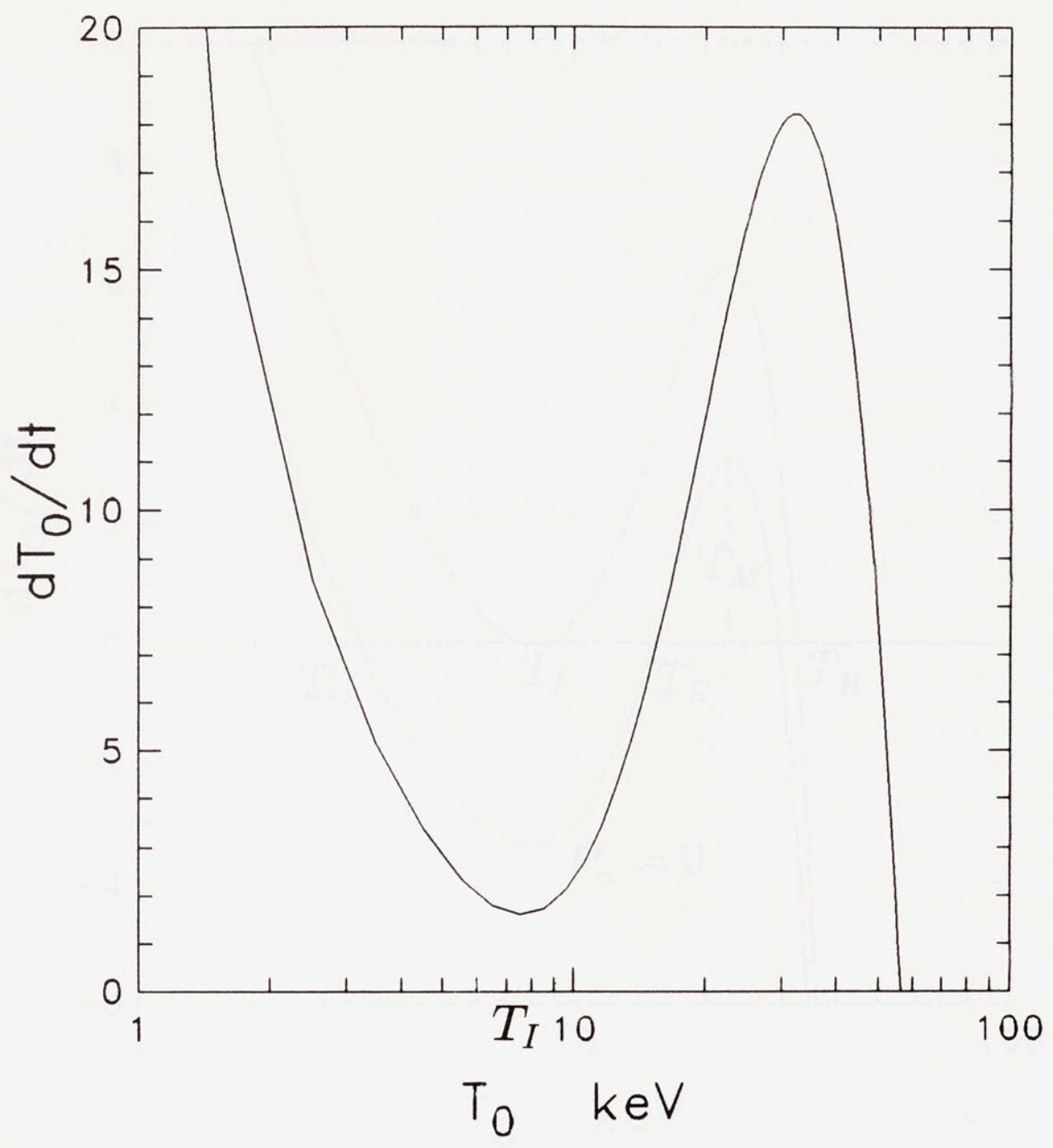

Figure 3.2: $\dot{T}$ vs $T$ plot illustrating the case of ohmic ignition 


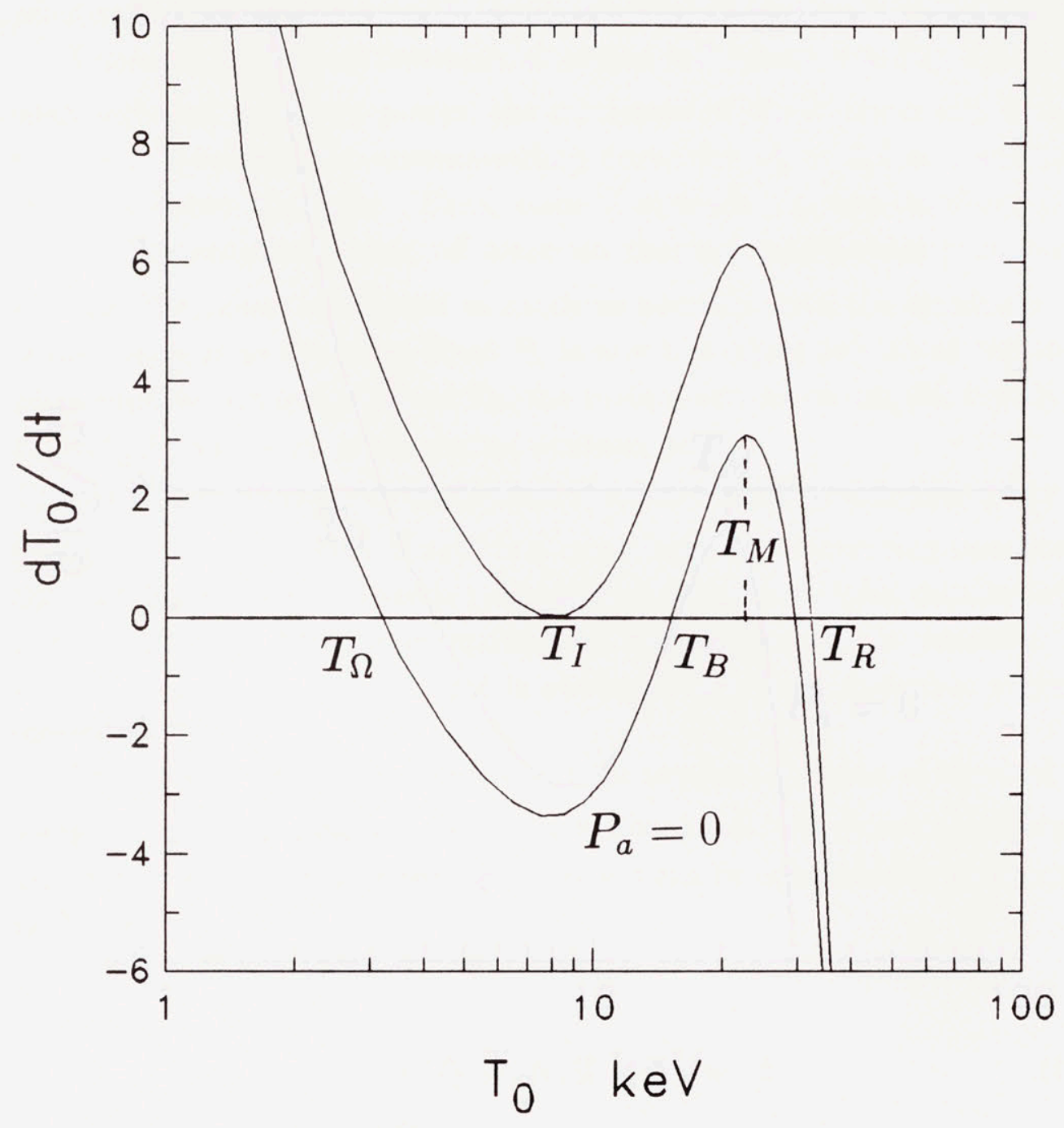

Figure 3.3: $\dot{T}$ vs $T$ plot illustrating the case of auxiliary power ignition 


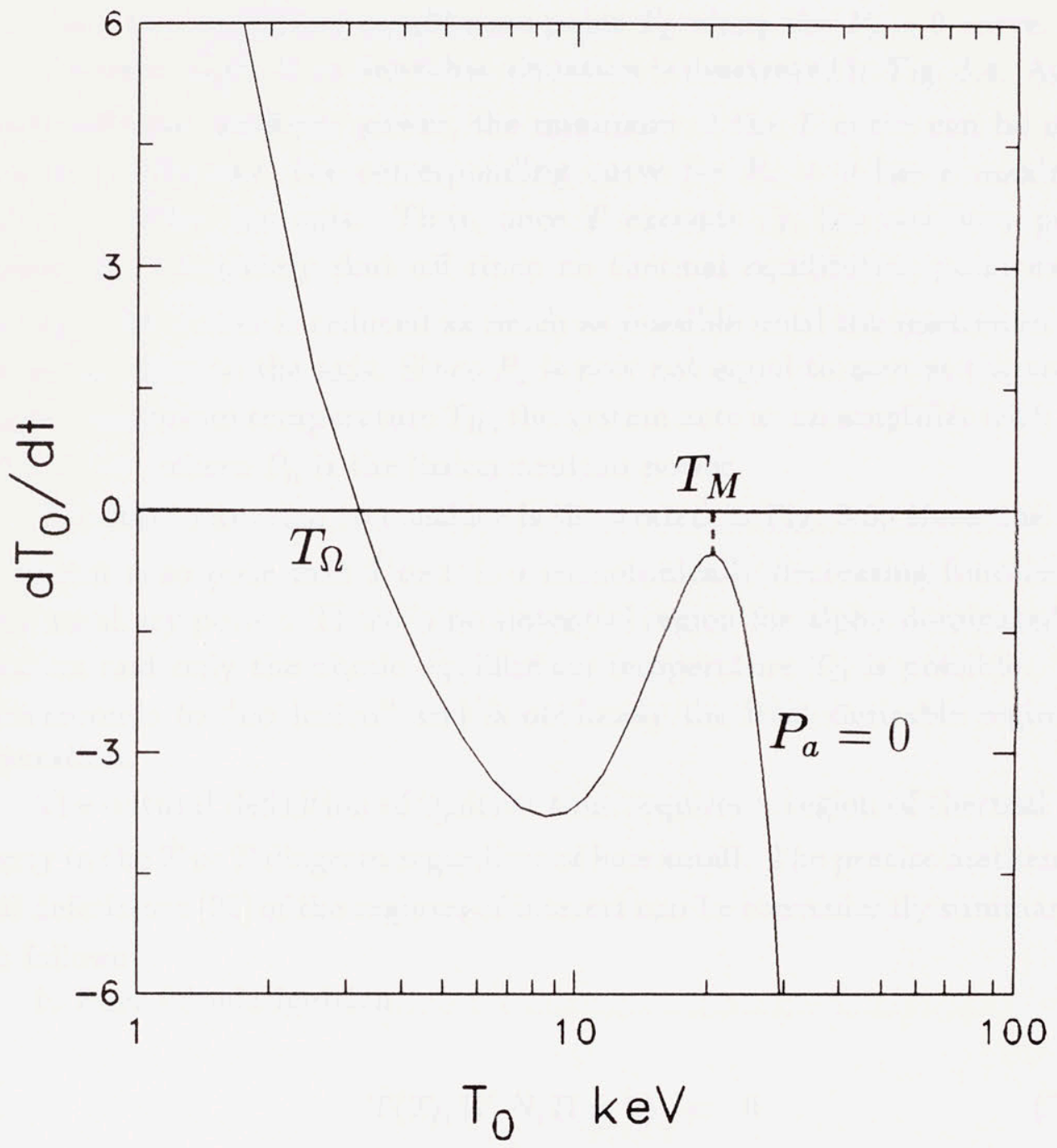

Figure 3.4: $\dot{T}$ vs $T$ plot illustrating the case of auxiliary power ignition in a low performance machine 
For non-ohmically ignited systems the form of the $\dot{T} v s T$ curve in Fig. 3 is the most desirable because, with the aid of auxiliary power, access is provided to the thermal equilibrium point $T_{B}$ along the $P_{a}=0$ curve.

A second, and not as desirable, situation is illustrated in Fig. 3.4. Again, with sufficient auxiliary power, the minimum of the $\dot{T}$ curve can be made positive. However, the corresponding curve for $P_{a}=0$ has a maximum which is below the axis. Thus, once $T$ exceeds $T_{I}$, the auxiliary power cannot be completely shut off since no thermal equilibrium point exists. Instead, the power is reduced as much as possible until the maximum of $\dot{T}$ is just tangent to the axis. Since $P_{a}$ is now not equal to zero at the steady state equilibrium temperature $T_{B}$, the system acts as an amplifier with gain $Q=P_{n} / P_{a}$ where $P_{n}$ is the fusion neutron power.

The third situation to consider is illustrated in Fig. 3.5. Here, the confinement is so poor that $\dot{T} v s T$ is a monotonically decreasing function for any auxiliary power. There is no potential region for alpha dominated operation and only the ohmic equilibrium temperature $T_{\Omega}$ is possible. This corresponds to "no fusion" and is obviously the least desirable regime of operation.

The natural definition of ignition thus requires a region of thermal runaway in the $\dot{T} v s T$ diagram regardless of how small. The precise mathematical definitions [23] of the regimes of interest can be conveniently summarized as follows

1. Pure Ohmic Ignition

$$
\begin{aligned}
\dot{T}\left(T_{I}, W, N, \Pi=0\right) & =0 \\
\frac{\partial \dot{T}}{\partial T}\left(T_{I}, W, N, \Pi=0\right) & =0
\end{aligned}
$$

2. Auxiliary Power Aided Ignition

$$
\begin{aligned}
\dot{T}\left(T_{I}, W, N, \Pi\right) & =0 \\
\frac{\partial \dot{T}}{\partial T}\left(T_{I}, W, N, \Pi\right) & =0 \\
\dot{T}\left(T_{M}, \Pi=0\right) & >0
\end{aligned}
$$




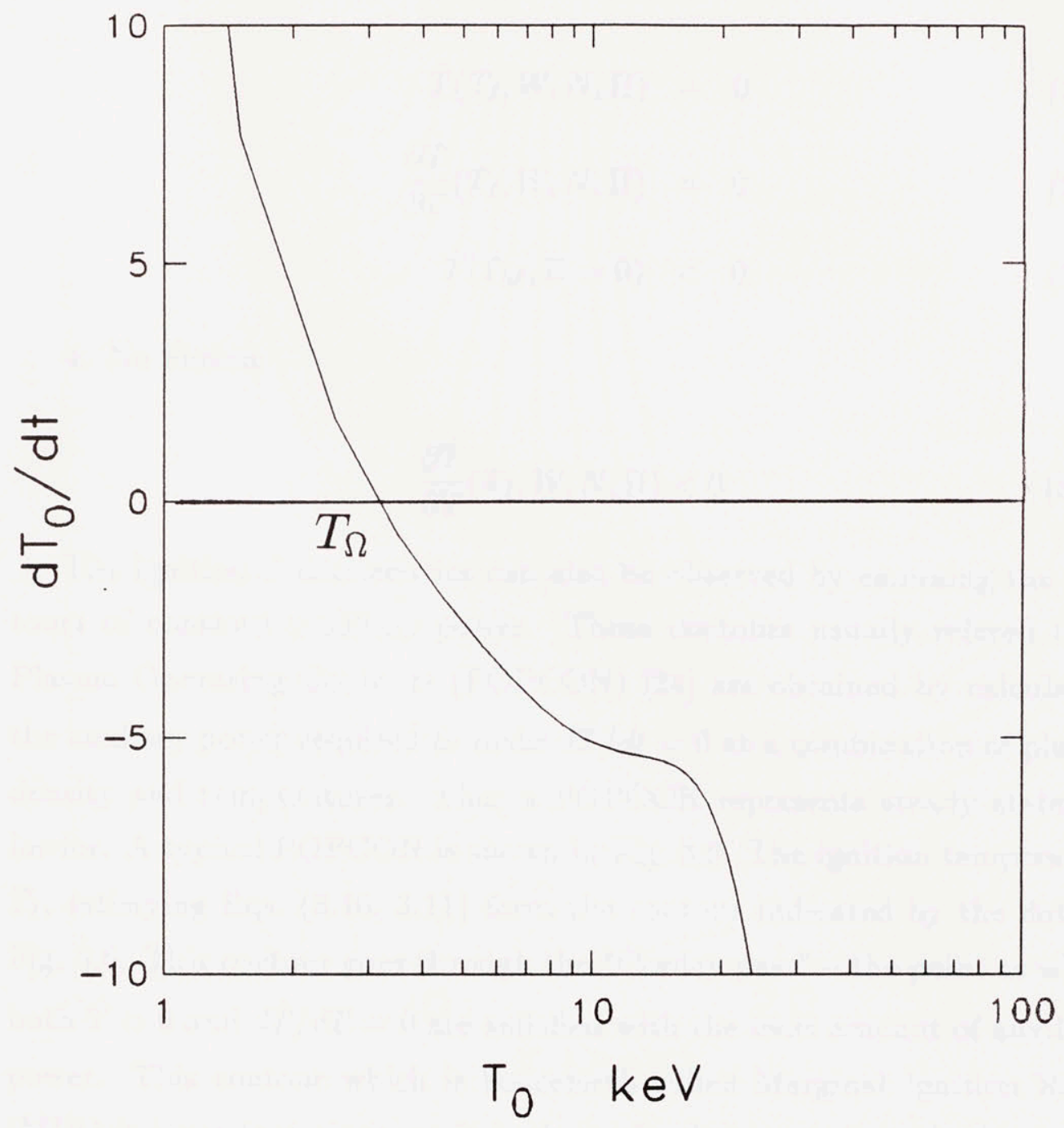

Figure 3.5: $\dot{T}$ vs $T$ plot illustrating the case of nonignited operation 
The significance of the temperature $T_{M}$ can be infered by examining Fig. 3.3.

3. Auxiliary Power Aided Amplification

$$
\begin{aligned}
\dot{T}\left(T_{I}, W, N, \Pi\right) & =0 \\
\frac{\partial \dot{T}}{\partial T}\left(T_{I}, W, N, \Pi\right) & =0 \\
\dot{T}\left(T_{M}, \Pi=0\right) & <0
\end{aligned}
$$

4. No Fusion

$$
\frac{\partial \dot{T}}{\partial T}\left(T_{I}, W, N, \Pi\right)<0
$$

The ignition characteristics can also be observed by eamining the contours of constant auxiliary power. These contours usually refered to as Plasma Operating Contours (POPCON) [24] are obtained by calculating the auxliary power required to make $d T / d t=0$ at a combination of plasma density and temperatures. Thus a POPCON represents steady state behavior. A typical POPCON is shown in Fig. 3.6. The ignition temperature $T_{I}$, satisfying Eqs. $(3.10,3.11)$ form the contour indicated by the dots in Fig. 3.6. This contour goes through the "Cordey pass" - the point at which both $\dot{T}=0$ and $d \dot{T} / d T=0$ are satisfied with the least amount of auxiliary power. This contour which is henceforth called Marginal Ignition Ridge (MIR) is important since, at it is shown in chapter 4 , it is the boundary between the regions of negative and positive growth rates for the plasma temperature

In the following section the ignition requirements for systems falling in regimes 1 and 2 are investigated, and the boundaries separating these regions in the parameter space of auxiliary power vs performance are presented. 


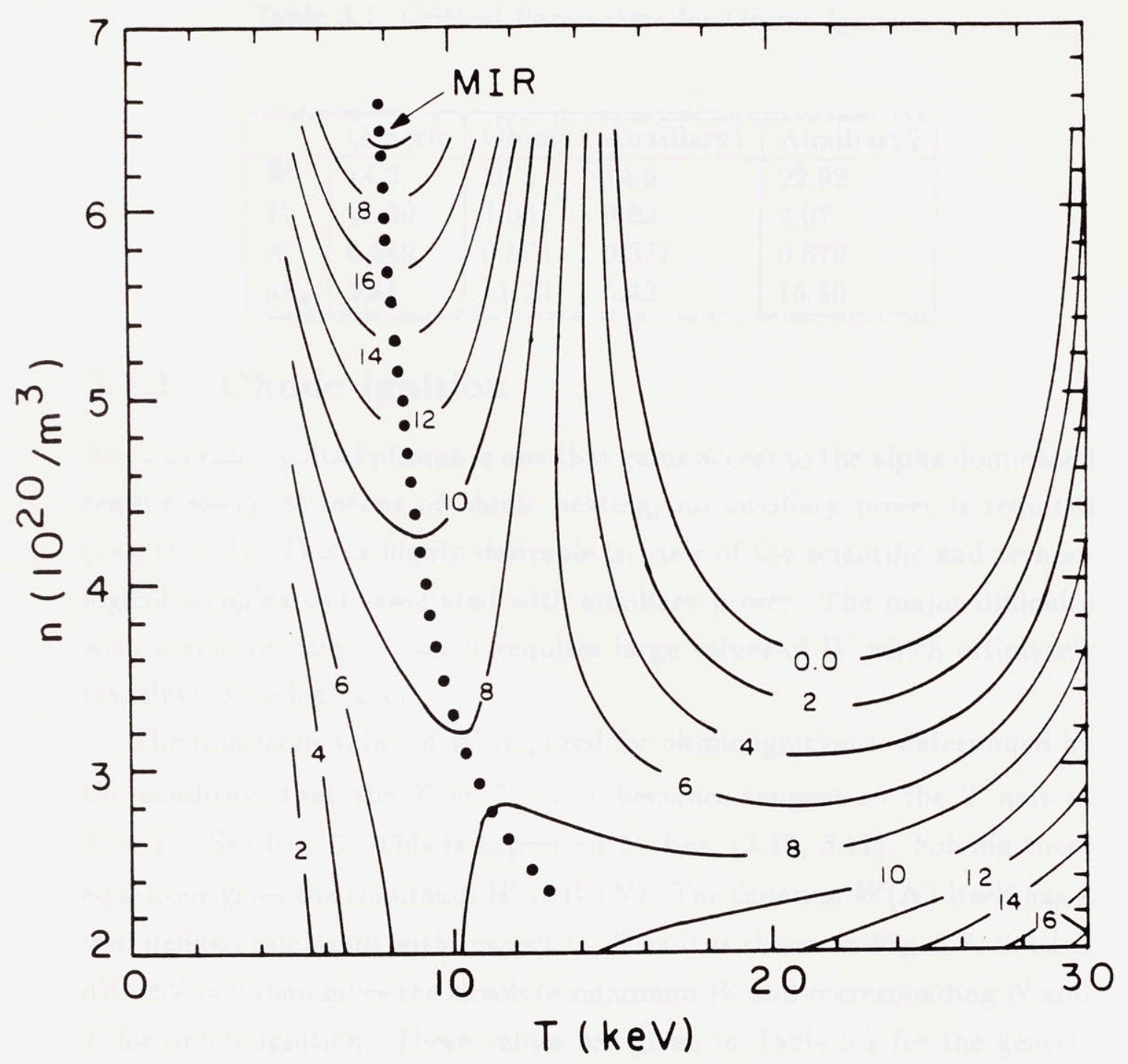

Figure 3.6: Typical POPCON plot indicating the Marginal Ignition Ridge (MIR) 
Table 3.1: Critical Parameters for Ohmic Ignition

\begin{tabular}{|l|l|l|l|l|}
\hline \hline \multicolumn{2}{|l|}{ Generic } & Ohmic & Auxiliary1 & Auxiliary2 \\
\hline$W$ & 14.2 & 19.5 & 14.6 & 22.92 \\
$T_{I}$ & 11.39 & 7.06 & 9.89 & 7.07 \\
$N$ & 0.249 & 0.576 & 0.371 & 0.679 \\
$n \tau_{E}$ & 3.51 & 11.20 & 5.42 & 15.56 \\
\hline \hline
\end{tabular}

\subsubsection{Ohmic Ignition}

An ohmically ignited plasma is one that gains access to the alpha dominated regime solely be means of ohmic heating; no auxiliary power is required (i.e., $\Pi=0$ ). This is highly desirable in view of the scientific and technological complexities associated with auxiliary power. The major difficulty with ohmic ignition is that it requires large values of $W$ which ultimately translates into high cost.

The minimum value of $W$ required for ohmic ignition is determined by the condition that the $\dot{T}$ vs $T$ curve becomes tangent to the $T$ axis at $T=T_{I}$. See Fig. 3. This is expressed by Eqs. $(3.10,3.11)$. Solving these equations gives the minimum $W=W(N)$. The function $W(N)$ itself has a well defined minimum with respect to $N$ as it is shown in Fig. 3.7. Setting $d W / d N=0$ then gives the absolute minimum $W$ and corresponding $N$ and $T$ for ohmic ignition. These values are given in Table 3.1 for the generic scalings, the ohmic scaling, and the auxiliary scaling. Also given are the values for $n \tau_{E}$.

By writting the function $\mathcal{F}$ in the general form

$$
\mathcal{F}=\frac{N^{\alpha}}{T^{\beta}}
$$

the complete parameter range of possible $N$ and $T$ dependances can be investigated. By taking $\alpha=0$ the minimum values of $W$ and the corresponding $N$ are plotted in Fig. 3.8 as a function of the exponent $\beta$. Note 


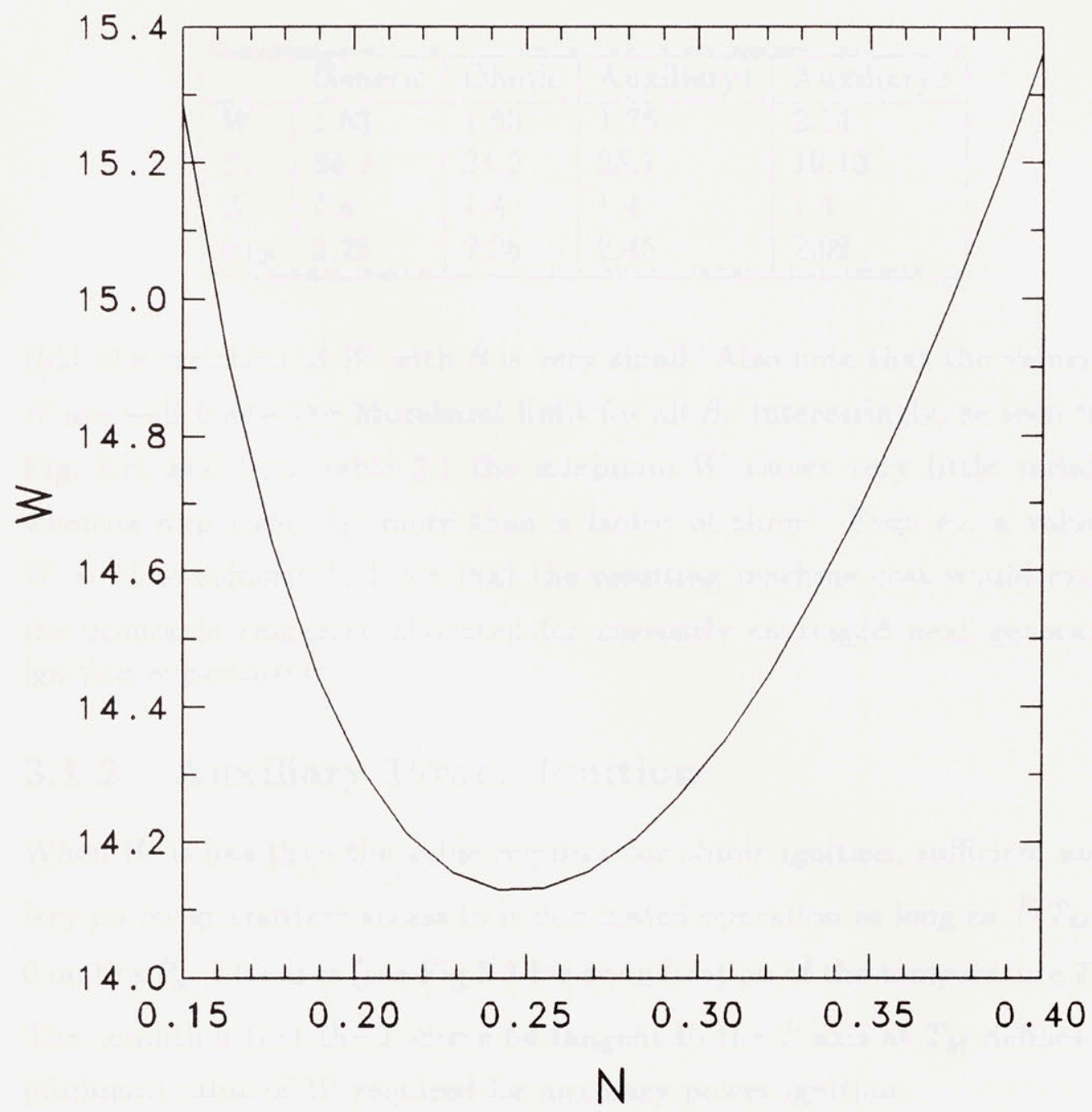

Figure 3.7: A plot of the performance paramenet $W$ as a function of the Murakami density factor $N$ 
Table 3.2: Critical Parameters for Auxiliary Power Ignition

\begin{tabular}{|l|l|l|l|l|}
\hline \hline & Generic & Ohmic & Auxiliary1 & Auxiliary2 \\
\hline$W$ & 1.63 & 1.63 & 1.75 & 2.14 \\
$T_{I}$ & 34.2 & 34.2 & 25.1 & 19.13 \\
$N$ & 1.4 & 1.4 & 1.4 & 1.4 \\
$n \tau_{E}$ & 2.28 & 2.28 & 2.45 & 2.99 \\
\hline \hline
\end{tabular}

that the variation of $W$ with $\beta$ is very small. Also note that the values for $N$ are well below the Murakami limit for all $\beta$. Interestingly, as seen from Fig. 3.8, and from table 3.1 the minimum $W$ shows very little variation whereas $n \tau_{E}$ varies by more than a factor of three. Even so, a value of $W \sim 15$ is sufficiently large that the resulting machine cost would exceed the economic resources allocated for currently envisaged next generation ignition experiments.

\subsubsection{Auxiliary Power Ignition}

When $W$ is less than the value required for ohmic ignition, sufficient auxiliary power guarantees access to $\alpha$ dominated operation as long as $\dot{T}\left(T_{M}\right)>$ 0 on the $P_{a}=0$ curve (see Fig 3.3 for an indication of the temperature $T_{M}$ ). The condition that the $\dot{T}$ curve be tangent to the $T$ axis at $T_{M}$ defines the minimum value of $W$ required for auxiliary power ignition.

The solution of Eqs. $(3.12,3.13)$ gives the minimum $W=W(N)$. In this regime $W$ monotonically decreases as $N$ increases. Thus, the absolute minimum value of $W$ occurs when $N$ reaches the Murakami limit. Table 3.2 gives the values of $W, T_{a}, N$ and $n \tau_{E}$ for the generic scaling, the ohmic scaling and the auxiliary scalings.

Observe that the value of $W$ is much smaller than that required for ohmic ignition. 

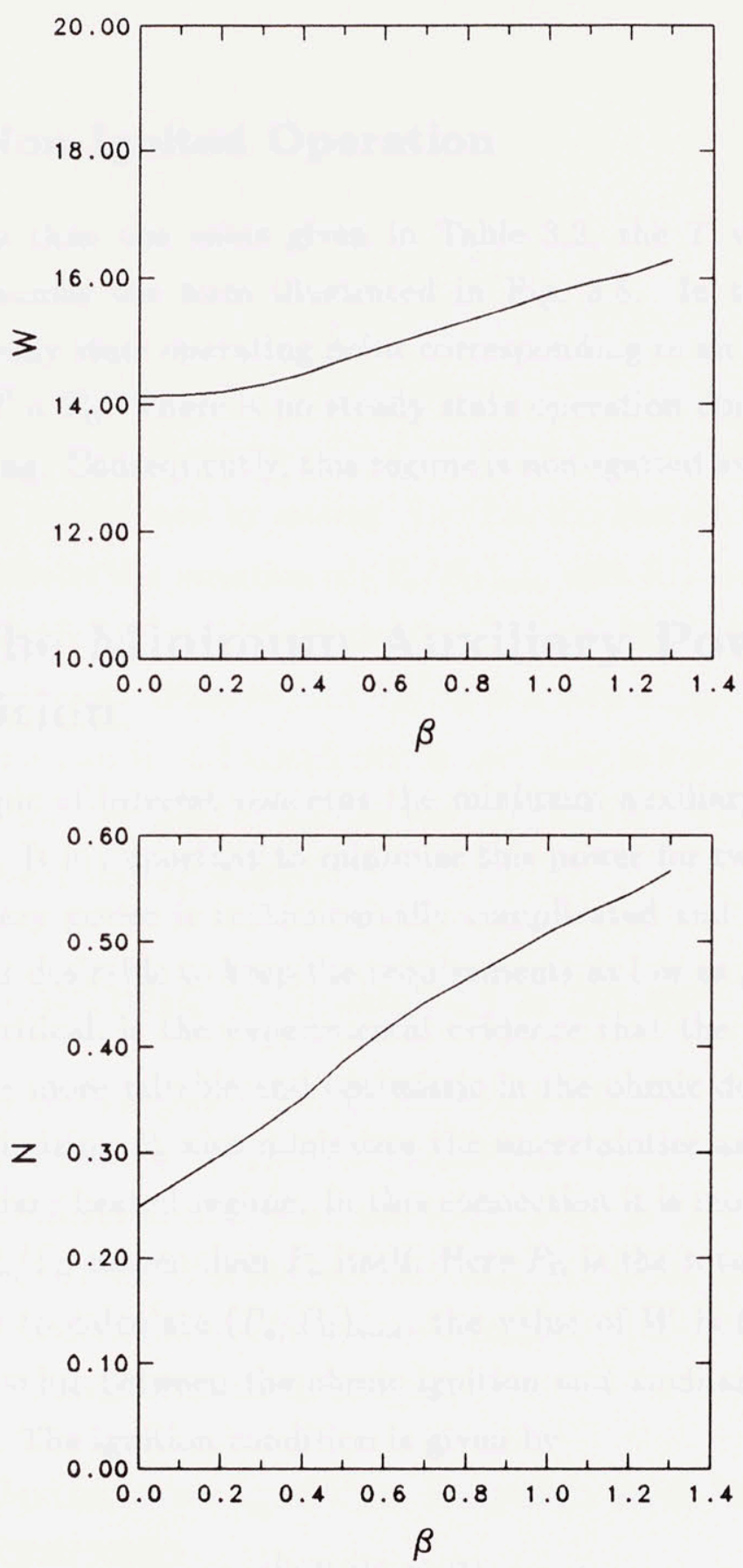

Figure 3.8: A plot of the optimum values for $W$ and $N$ as a function of the exponent $\beta$ in Eq. (3.19) 


\subsubsection{Non-Ignited Operation}

If $W$ is less than the value given in Table 3.2 , the $\dot{T}$ vs $T$ diagram for $P_{a}=0$ assumes the form illustrated in Fig. 3.5. In this case there is only one steady state operating point corresponding to an ohmically heated discharge, $T=T_{\Omega}$. There is no steady state operation dominated by alpha power heating. Consequently, this regime is non-ignited and is discussed no further.

\subsection{The Minimum Auxiliary Power for Ig- nition}

Another topic of interest concerns the minimum auxiliary power required for ignition. It is important to minimize this power for two reasons. First, since auxiliary power is technologically complicated and economically expensive, it is desirable to keep the requirements as low as possible. Second, and more critical, is the experimental evidence that the empirical scaling relations are more reliable and optimistic in the ohmic dominated regime. Hence, minimizing $P_{a}$ also minimizes the uncertainties associated with $\tau_{E}$ in the auxiliary heated regime. In this connection it is more appropriate to minimize $P_{a} / P_{\Omega}$ rather than $P_{a}$ itself. Here $P_{\Omega}$ is the total ohmic power.

In order to calculate $\left(P_{a} / P_{\Omega}\right)_{\text {min }}$, the value of $W$ is fixed at some intermediate value between the ohmic ignition and auxiliary power ignition boundaries. The ignition condition is given by

$$
\begin{aligned}
\dot{T}(T, W, N, \Pi) & =0 \\
\frac{\partial \dot{T}}{\partial T}(T, W, N, \Pi) & =0
\end{aligned}
$$

Solving these equations gives $\Pi=\Pi(N, W)$. This is substituted into the following 


$$
\Gamma \equiv \frac{P_{a}}{P_{\Omega}}=\frac{C_{a}}{C_{\Omega}} \Pi T^{3 / 2}
$$

yielding $\Gamma=\Gamma(N, W)$. For higher values of $W, \Gamma$ has a minimum with respect to $N$ (see Fig 3.7) that is less than the Murakami limit. At smaller $W$, the minimizing $N$ exceeds the Murakami limit. In this situation the minimum $W$ is obtained by setting $N=1.4$, the Murakami limit.[22]

Fig. 3.9 shows the variation of $\left(P_{a} / P_{\Omega}\right)_{\min }$ with $W$. As expected $P_{a} / P_{\Omega}$ is a decreasing function of $W$, illustrating the basic tradeoff between physics reliability and cost. Observe that $P_{a} / P_{\Omega}$ is a very steep function of $W$ for small $W$. Thus, for $W<5$ the plasma is operating in a particularly sensitive region of parameter space. Also there is relatively small variation in $P_{a} / P_{\Omega}$ for the different scaling relations.

\subsection{Coupling Physics and Engineering}

Up to this point the goal was to estimate the values of the various physics based parameters $W, N, \Pi$. These parameters are directly related to the physics considerations as represented by the 0 -D model. In this section the ignition ideas developed in the previous sections are coupled to fundamental engineering considerations of both the toroidal and the ohmic heating coils resulting in optimized tokamak designs.

\subsubsection{Optimized Ignition Physics and Machine Per- formance}

The first step is to develop a sharply defined goal of "optimized ignition". There are two main issues to consider. First, since we have been driven to auxiliary power aided ignition because of the prohibitive economic limitations imposed by pure ohmic ignition, the total machine cost is clearly one of the most important problems. Second, and somewhat less quantitative, 


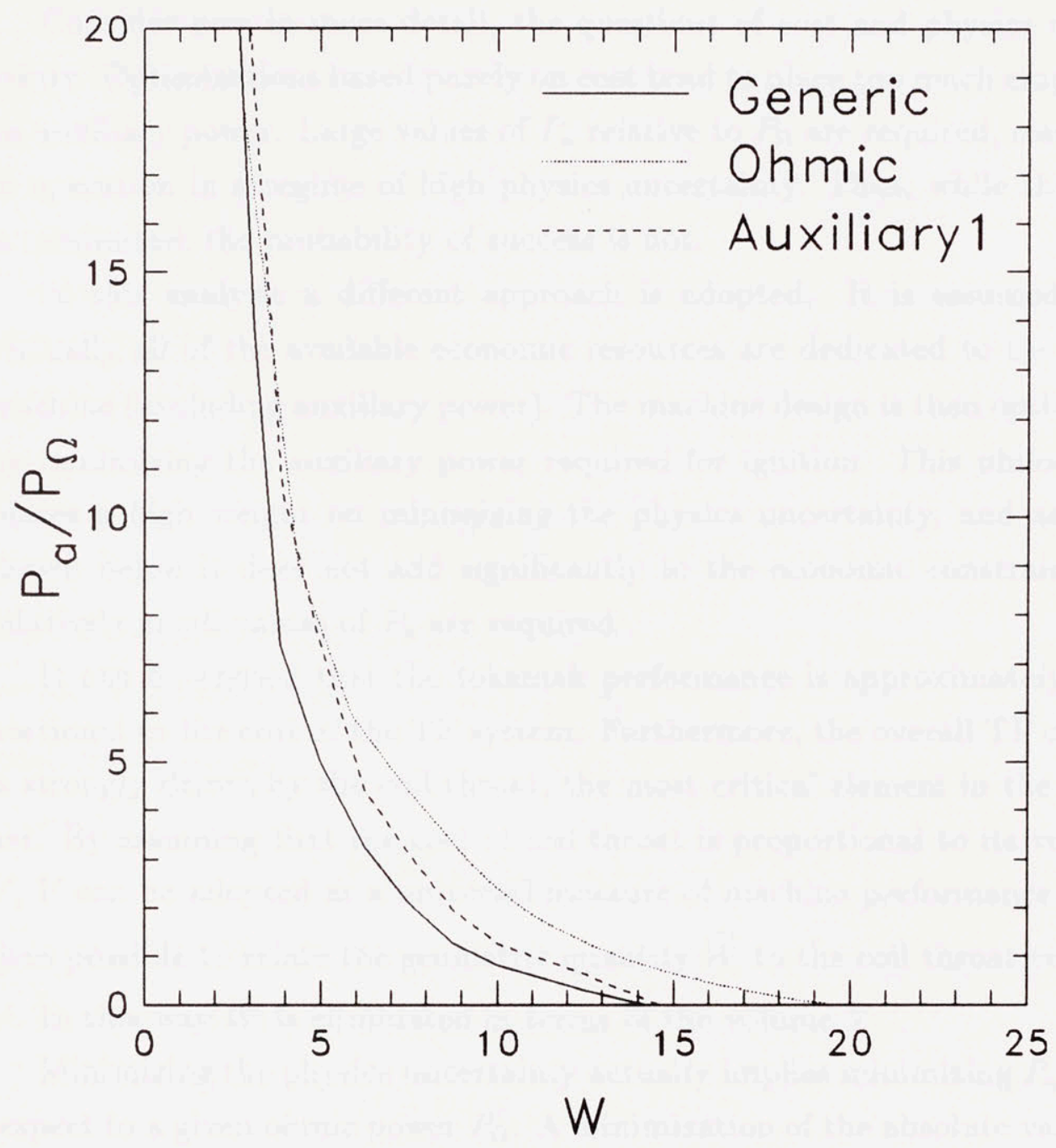

Figure 3.9: A plot of the optimum value of $P_{a} / P_{\Omega}$ as a function of the paraneter $W$ 
is the relatively high physics uncertainty associated with auxiliary power confinement scaling as compared to the ohmic scaling.

Consider now in more detail, the questions of cost and physics uncertainty. Optimizations based purely on cost tend to place too much emphasis on auxiliary power. Large values of $P_{a}$ relative to $P_{\Omega}$ are required, resulting in operation in a regime of high physics uncertainty. Thus, while the cost is minimized, the probability of success is not.

In this analysis a different approach is adopted. It is assumed that virtually all of the available economic resources are dedicated to the basic machine (excluding auxiliary power). The machine design is then optimized by minimizing the auxiliary power required for ignition. This philosophy places a high weight on minimizing the physics uncertainty, and as it is shown below it does not add significantly to the economic constraints as relatively small values of $P_{a}$ are required.

It can be argued that the tokamak performance is approximately proportional to the cost of the TF system. Furthermore, the overall TF design is strongly driven by the coil throat, the most critical element in the magnet. By assuming that the cost of coil throat is proportional to its volume $V, V$ can be adopted as a universal measure of machine performance. It is then possible to relate the geometric quantity $\widehat{W}$ to the coil throat volume $V$. In this way $\widehat{W}$ is eliminated in terms of the volume $V$

Minimizing the physics uncertainty actually implies minimizing $P_{a}$ with respect to a given ohmic power $P_{\Omega}$. A minimization of the absolute value of $P_{a}$ leads to the unphysical limit $B_{0} \rightarrow \infty, a \rightarrow 0$. Since $a \rightarrow 0$, the plasma volume approaches zero implying that no auxiliary power is required. Even so, in this limit, the ratio $P_{a} / P_{\Omega}$ can be large implying high physics uncertainty.

Thus, in the analysis that follows, the quantity $P_{a} / P_{\Omega}$ required for ignition is minimized, subject to the constraint of a fixed volume $V$ set at the maximum value permissable by economic constraints. When the calculation is complete it is shown a posteriori that the auxiliary power cost is 
not prohibitive and that the equilibrium burn temperature is indeed in the ignited regime of operation.

The optimization procedure can be summarized mathematically as follows. The quantity $P_{a} / P_{\Omega}$ is easily found from Eq. (3.1).

$$
\frac{P_{a}}{P_{\Omega}}=\frac{C_{a}}{C_{\Omega}} \Pi T^{3 / 2} \equiv U\left(T, \Pi, N, W, q_{*}, \kappa, a, R_{0}\right)
$$

$U$ is to be minimized subject to the condition that the plasma is ignited.

$$
\begin{aligned}
\dot{T}\left(T_{I}, \Pi_{I}, N, W, q_{*}, \kappa\right) & =0 \\
\frac{\partial \dot{T}}{\partial T}\left(T_{I}, \Pi_{I}, N, W, q_{*}, \kappa\right) & =0
\end{aligned}
$$

and the constraint that the TF coil throat volume $V$ is fixed. In the next section it is shown that $V=V\left(\widehat{W}, a, R_{0}, \kappa, q_{*}\right)$ so that the fixed volume constraint becomes

$$
V\left(\widehat{W}, a, R_{0}, \kappa, q_{*}\right)=\text { const. }
$$

In principle, Eqs. (3.24) and (3.25) are solved for $T_{I}$ and $\Pi_{I}$, and Eq. (3.26) for $\widehat{W}$. These results are substituted into Eq. (3.23) yielding

$$
U=U\left(N, q_{*}, \kappa, a, R_{0} ; V\right)
$$

The quantity $U$ is then minimized with respect to $N, q_{*}, \kappa, a, R_{0}$ holding $V$ fixed. In practice, the dependence of $U$ on $q_{*}$ and $\kappa$ is monotonic implying that these quantities must be set to their extremum values as determined by physics: $q_{*}=2, \kappa=2$. What remains is a three dimensional minimization of $U$ with respect to $N, a, R_{0}$. 


\subsubsection{TF Coil Design}

The one remaining relation required is that expressing the throat volume, $V$, in terms of the ignition parameters. The coil model described below is sufficient for this purpose. The critical assumption is that $V$ is determined by stress considerations on the inner leg of the magnet.

The coil model is deliberately simplified for purposes of pedagogical clarity and to avoid any false impressions of unjustified accuracy. It is important to realize that the goal of this work is to demonstrate trends rather than to arrive at a precise final design. Thus, while the results are only semi-quantitatively correct in detail, they quite accurately describe the features and directions of the optimized design.

Consider the rectangular coil model illustrated in Fig. 3.10. The distances $b_{1}, b_{2}$ and $b_{3}$ separate the plasma from the TF magnet and provide space for the first wall, divertor, RF antennae, etc. The values of $b_{1}$ and $b_{2}$ are taken from the current CIT design and are assumed to be known quantities. The value of $b_{3}$ is scaled with the minor radius according to the relation. [25]

$$
b_{3}=0.88 a+0.5
$$

The thickness of the coil throat is denoted by $c$, and it is this quantity that is crucial in determining the volume $V$. The value of $c$ is found by calculating the Tresca stress on the inner leg of the magnet and setting it equal to its maximum allowable value $\sigma_{m} \approx 500 \mathrm{MPa}$.

The analysis begins with the evaluation of $V$. A short calculation gives

$$
V=4 \pi c\left(\kappa a+b_{3}\right)\left(R_{0}-a-b_{1}-c / 2\right)
$$

In order to obtain a reference case, for comparing the accuracy of the model, the CIT parameters are considered (i.e. $R_{0}=2.1 \mathrm{~m}, a=.64 \mathrm{~m}, \kappa=2.0$, $b_{1}=.17 \mathrm{~m}, b_{2}=.6 \mathrm{~m}, b_{3}=.86 \mathrm{~m}, \mathrm{c}=.43 \mathrm{~m}, B_{0}=11 \mathrm{~T}, I=11 \mathrm{MA}$, $\sigma_{m}=500 \mathrm{MPa}$ ). This gives a throat volume $V=12.4 \mathrm{~m}^{3}$ for the reference case. 


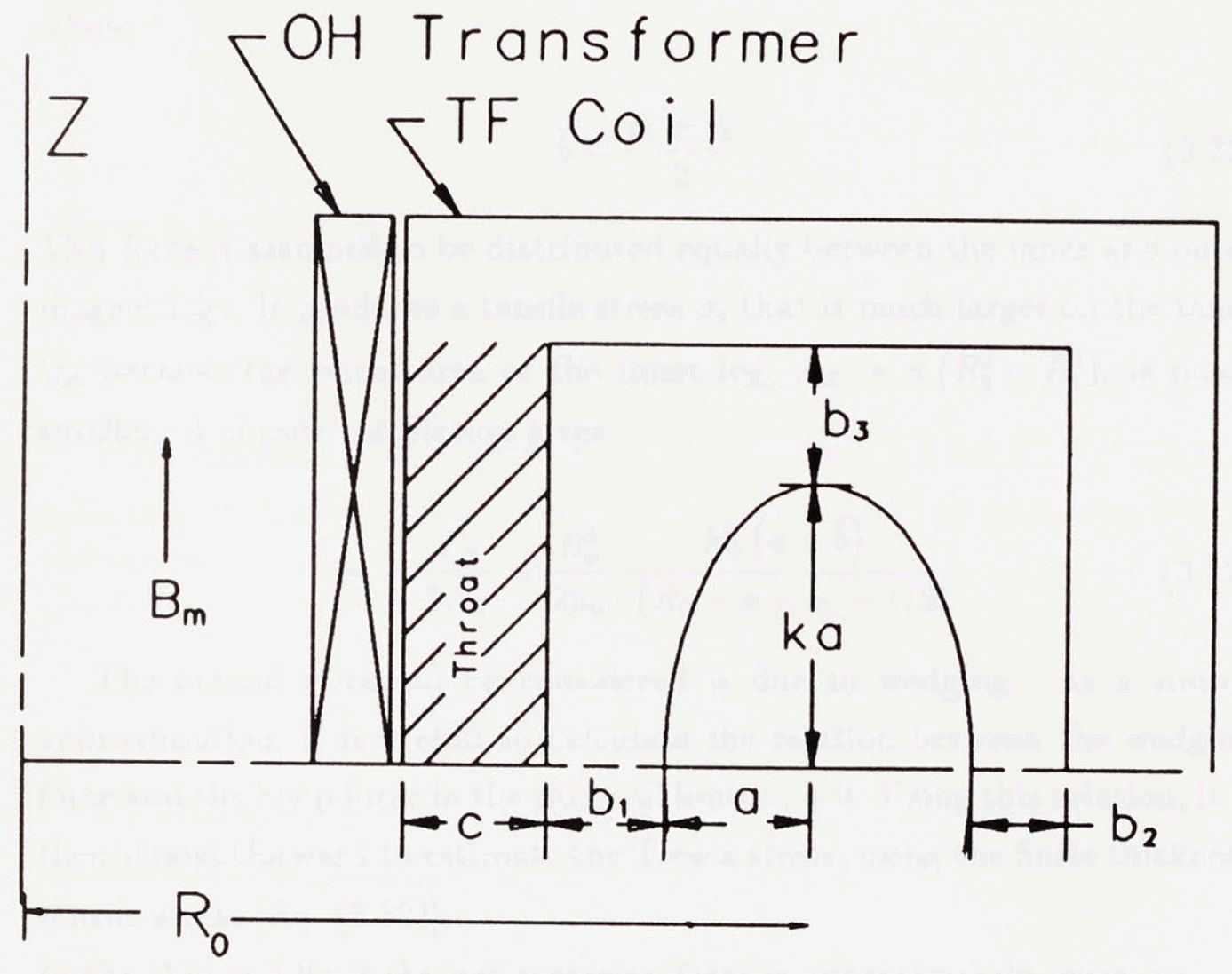

Figure 3.10: A toroidal coil model 
Next, consider the vertical hoop stress. The total vertical force $F_{Z}$ acting on the upper half of the magnet can be approximated by

$$
F_{Z} \approx \frac{2 \pi}{\mu_{0}} B_{0}^{2} R_{0}(a+\bar{b})
$$

where

$$
\bar{b}=\frac{b_{1}+b_{2}}{2}
$$

This force is assumed to be distributed equally between the inner and outer magnet legs. It produces a tensile stress $\sigma_{t}$ that is much larger on the inner leg because the stress area of the inner leg, $A_{Z}=\pi\left(R_{2}^{2}-R_{1}^{2}\right)$, is much smaller. A simple calculation gives

$$
\sigma_{t}=\frac{F_{Z}}{2 A_{Z}}=\frac{B_{0}^{2}}{2 \mu_{0}} \frac{R_{0}(a+\bar{b})}{c\left(R_{0}-a-b_{1}-c / 2\right)}
$$

The second stress to be considered is due to wedging. As a simple approximation, it is usefull to calculate the relation between the wedging force and the hoop force in the thin coil limit $c \rightarrow 0$. Using this relation, it is then straightforward to estimate the Tresca stress, using the finite thickness tensile stress [Eq. (3.32)].

In the thin coil limit the net centering force is approximately given by

$$
F_{R} \simeq-\frac{2 \pi}{\mu_{0}} B_{0}^{2} R_{0}^{2}\left(\kappa a+b_{3}\right)\left[\frac{1}{R_{0}-a-b_{1}}-\frac{1}{R_{0}+a+b_{2}}\right]
$$

The centering force $F_{R}$ produces a wedging stress

$$
\sigma_{w}=\frac{F_{R}}{2 \pi A_{R}}
$$

where $A_{R} \approx 2\left(\kappa a+b_{3}\right) c$. Substituting Eq. (3.33) into Eq. (3.34) gives 


$$
\sigma_{w} \approx-\frac{B_{0}^{2} R_{0}^{2}}{2 \mu_{0} c}\left[\frac{1}{R_{0}-a-b_{1}}-\frac{1}{R_{0}+a+b_{2}}\right]
$$

In the thin coil limit, a comparison of Eqs. (3.32) and (3.35) indicates that

$$
\left.\frac{\sigma_{w}}{\sigma_{t}}\right|_{c \rightarrow 0}=-\frac{2}{1+\epsilon_{2}}
$$

Where $\epsilon_{2}$ is given by

$$
\epsilon_{2}=\frac{a+b_{2}}{R_{0}}
$$

Now assume for simplicity that Eq. (3.36) is valid for finite thickness coils as well. Under this assumption, the Tresca stress $\sigma_{T} \equiv \sigma_{t}-\sigma_{w}$ can be written as

$$
\sigma_{T}=\frac{B_{0}^{2}}{2 \mu_{0}}\left(\frac{3+\epsilon_{2}}{1+\epsilon_{2}}\right) \frac{(a+\bar{b}) R_{0}}{c\left(R_{0}-a-b_{1}-c / 2\right)}
$$

By setting $\sigma_{T}=\sigma_{m}$ (the maximum allowable stress) in Eq. (3.38) the coil thickness is given by

$$
c=\frac{B_{0}^{2}}{2 \mu_{0} \sigma_{m}}\left(\frac{3+\epsilon_{2}}{1+\epsilon_{2}}\right) \frac{(a+\bar{b}) R_{0}}{c\left(R_{0}-a-b_{1}-c / 2\right)}
$$

By substituting Eq. (3.39) into Eq. (3.29) the coil volume is given by

$$
V=\frac{\pi B^{2} R_{0}}{\mu_{0} \sigma_{m}}\left\{\left[\frac{1}{\left(2 a+b_{1}+b_{2}\right)\left(\kappa a+b_{3}\right)}\right]\left[\frac{R_{0}+a+b_{2}}{\left(3 R_{0}+a+b_{2}\right)}\right]\right\}^{-1}
$$

Equation (3.40) is the desired relation. It is the equation that couples the ignition physics and the engineering. Since the parameter $\widehat{W}$ depends on the 
plasma geometry the coil volume given by Eq. (3.40) can alternatively be expressed as a function of $\widehat{W}$. In order to check the calibration of this model the CIT parameters are used. By solving Eq. (3.40) for $B$ and substituting the CIT parameters gives $B=10.7$ Tesla which is in good agreement with the 11 Tesla CIT design.

In practice there are several important limits which prevent small $R_{0}$. Certainly, the geometric condition $R_{0}>a+b_{1}+c$ must be satisfied. An even stronger limit on $R_{0}$ results from the volt-second requirements of the ohmic transformer, and it is investigated next.

\subsubsection{OH Coil Design: Volt Second Requirements.}

In this section a derivation of the minimum major radius that satisfies the volt-second requirements of the ohmic transformer is presented.

\subsubsection{Basic Constraint}

The basic constraint defining the volt-second requirements can be written as

$$
\Delta \psi=k L I
$$

Here, $\Delta \psi$ is the flux swing in the transformer, $I$ is the plasma current, and $L$ is the plasma inductance, which can be approximated as [26]

$$
L=\mu_{0} R_{0}\left[\ln \left(\frac{8 R_{0}}{a \kappa^{1 / 2}}\right)-\frac{7}{4}\right]
$$

The parameter $k$ is a constant and typically $k \approx 1.2$. This guarantees sufficient volt-seconds to bring the current from zero to its final value as well as maintaining the plasma for an extended period of flat top operation.

In equation (3.41) it is also convenient to express $I$ in terms of $B_{0}$ and $q_{*}$

$$
I=\frac{2 \pi a^{2} \kappa B_{0}}{\mu_{0} R_{0} q_{*}}
$$




\subsubsection{Evaluation of $\Delta \psi$}

In order to evaluate $\Delta \psi$ it is assumed that the magnetic field in the transformer is double swung from $-B_{m}$ to $+B_{m}$. For a double swing system

$$
\Delta \psi=2\left(\psi_{1}+\psi_{2}\right)
$$

where $\psi_{1}$ is the flux in the vacuum region and $\psi_{2}$ is the flux in the coil. The final value of the flux in the vacuum region is

$$
\psi_{1}=\pi R_{a}^{2} B_{m}
$$

where the transformer geometry is illustrated in Fig. 3.11. The flux in the coil itself is easily found by noting that for a uniform current density

$$
B=B_{m}\left(\frac{R_{b}-R}{R_{b}-R_{a}}\right) \quad R_{a}<R<R_{b}
$$

A simple calculation then gives

$$
\psi_{2}=\frac{\pi B_{m}}{3}\left(R_{b}-R_{a}\right)\left(R_{b}+2 R_{a}\right)
$$

Therefore $\Delta \psi$ becomes

$$
\Delta \psi=2 \pi B_{m}\left[R_{a}^{2}+\frac{1}{3}\left(R_{b}-R_{a}\right)\left(R_{b}+2 R_{a}\right)\right]
$$

\subsubsection{Relate the maximum field $B_{m}$ to the maximum stress $\sigma_{p}$} Typically $\sigma_{p} \approx 330 \mathrm{MPa}$ for CIT. The local Tresca stress in a straight solenoid has the form[27]

$$
\sigma_{T}(R)=\frac{B_{m}^{2}}{\mu_{0}}\left(\frac{R_{a}^{2}}{R_{b}^{2}-R_{a}^{2}}\right)\left(\frac{R_{b}^{2}}{R^{2}}\right)
$$

Unlike the TF coil, $\sigma_{T}$ is a strong function of $R$ in the ohmic transformer coil, particularly if the coil is thick. 


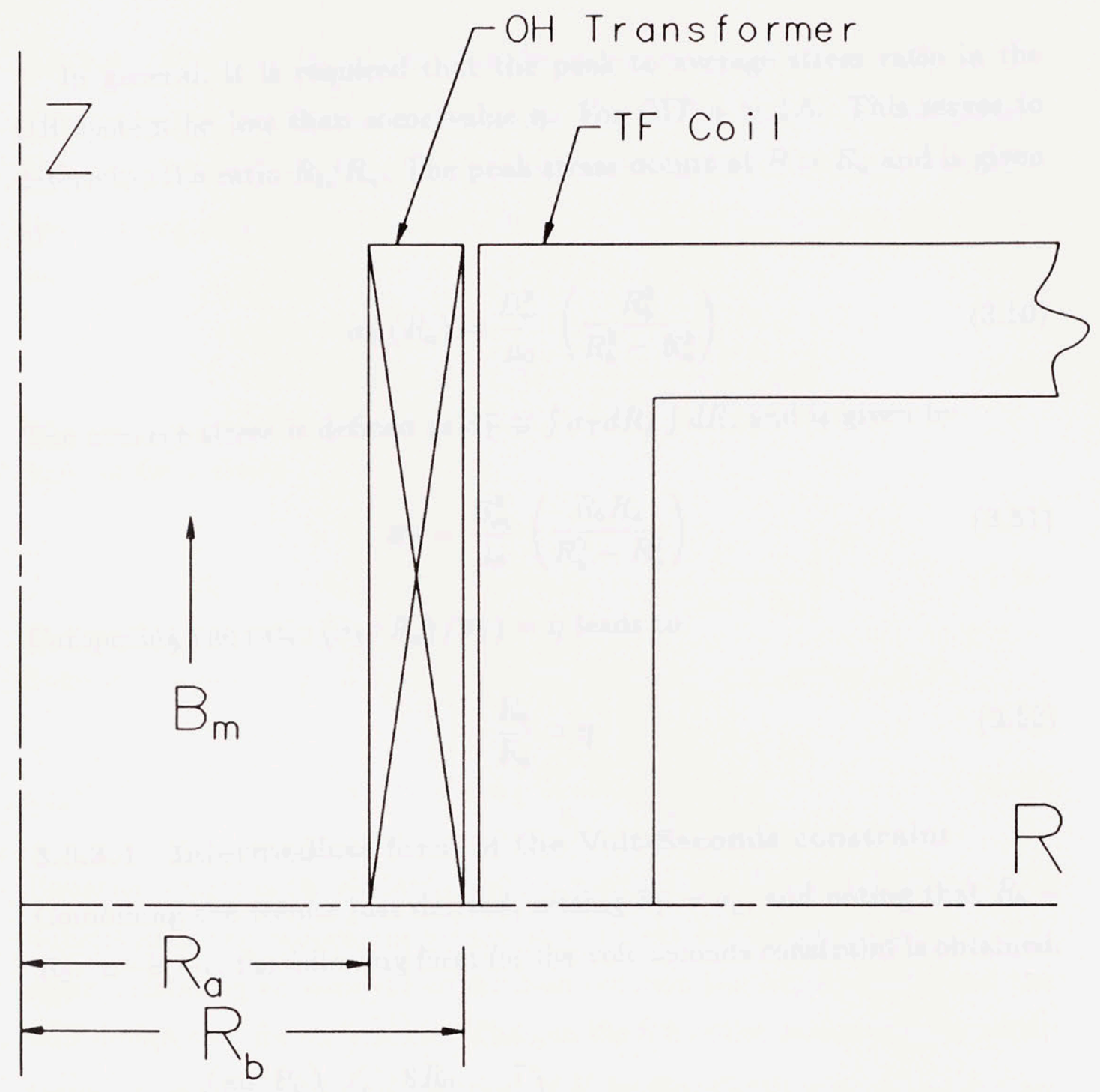

Figure 3.11: The ohmic heating coil model 
In general, it is required that the peak to average stress ratio in the $\mathrm{OH}$ system be less than some value $\eta$. For CIT $\eta \simeq 1.5$. This serves to determine the ratio $R_{b} / R_{a}$. The peak stress occurs at $R=R_{a}$ and is given by

$$
\sigma_{T}\left(R_{a}\right)=\frac{B_{m}^{2}}{\mu_{0}}\left(\frac{R_{b}^{2}}{R_{b}^{2}-R_{a}^{2}}\right)
$$

The average stress is defined as $\overline{\sigma_{T}} \equiv \int \sigma_{T} d R / \int d R$, and is given by

$$
\overline{\sigma_{T}}=\frac{B_{m}^{2}}{\mu_{0}}\left(\frac{R_{b} R_{a}}{R_{b}^{2}-R_{a}^{2}}\right)
$$

Computing the ratio $\left(\sigma_{T}\left(R_{a}\right) / \overline{\sigma_{T}}\right)=\eta$ leads to

$$
\frac{R_{b}}{R_{a}}=\eta
$$

\subsubsection{Intermediate form of the Volt-Seconds constraint}

Combining the results just derived, setting $\overline{\sigma_{T}}=\sigma_{p}$, and noting that $R_{b}=$ $R_{0}-a-b_{1}-c$, the following form for the volt-seconds constraint is obtained.

$$
\begin{aligned}
& k\left(\frac{\kappa a^{2} B_{0}}{q_{*}}\right)\left(\ln \frac{8 R_{0}}{a \kappa^{1 / 2}}-\frac{7}{4}\right) \\
& =\left(\mu_{0} \sigma_{p}\right)^{1 / 2}\left(\frac{\eta^{2}-1}{\eta}\right)^{1 / 2}\left[\frac{\eta^{2}+\eta+1}{3 \eta^{2}}\right]\left(R_{0}-a-b_{1}-c\right)^{2}(3.5)
\end{aligned}
$$

Substituting the values $\sigma_{p}=330 M P a, \eta=1.5, k=1.2$ leads to

$$
R_{0}=a+b_{1}+c+.4\left(\frac{\kappa B a^{2}}{q_{*}}\right)^{1 / 2}
$$




\subsubsection{Final form of the Volt-Seconds constraint}

The final step in the calculation is the elimination of the coil thickness $c$ from Eq. (3.54). The quantity $c$ is expressed in terms of $V$ from Eq. (3.29), the defining relation for the coil throat volume. Equation (3.29) can be written as

$$
c^{2}-2\left(R_{0}-a-b_{1}\right) c+\frac{V}{2 \pi\left(\kappa a+b_{3}\right)}=0
$$

Solving for $c$ yields

$$
c=R_{0}-a-b_{1}-\left[\left(R_{0}-a-b_{1}\right)^{2}-\frac{V}{2 \pi\left(\kappa a+b_{3}\right)}\right]^{1 / 2}
$$

Substituting into Eq. (3.54) leads to the following expression for $R_{0}$

$$
R_{0}=a+b_{1}+0.4\left(\frac{\kappa a^{2} B}{q_{*}}+\frac{V}{\kappa a+b_{3}}\right)^{1 / 2}
$$

Equation (3.57) is the desired relation, giving $R_{0}=R_{0}\left(B, a, V, \kappa, q_{*}\right)$.

Substituting the calibration values on the right hand side gives $R_{0}=2.1$, again indicating an accurate calibration between the simple model and the CIT design used for calibration. Thus, in the full optimization the geometric factor $\widehat{W}$ is expressed as a function of $V$ by Eq. (3.40) with $R_{0}$ evaluated from Eq. (3.57).

\subsection{Results}

The physics and the engineering are coupled through Eqs. (3.24, 3.25, 3.40, 3.54). Eqs. (3.24 and 3.25) represent the ignition condition, and Eqs. (3.40, 3.57) characterize the toroidal field and ohmic heating coils. The optimized desigs are obtained by simultaneously solving Eqs. (3.24, 3.25, 3.40, 3.54) and by minimizing the quantity 
Table 3.3: The Parameters $\alpha_{j}$ corresponding to Eq. (3.59) for various confinement scalings

\begin{tabular}{|l|c|c|c|c|c|}
\hline \hline \multicolumn{2}{|c|}{$\alpha_{1}$} & $\alpha_{2}$ & $\alpha_{3}$ & $\alpha_{4}$ & $\alpha_{5}$ \\
\hline Goldston & 1.75 & -0.37 & 0.0 & 1.0 & 0.5 \\
Neo-Alcator & 1.0 & 1.0 & 1.0 & 0.0 & 0.5 \\
Kaye-Goldston & 1.39 & -0.49 & 0.17 & 1.24 & 0.28 \\
ITER & 1.1 & 0.3 & 1.2 & 0.85 & 0.5 \\
\hline \hline
\end{tabular}

$$
\frac{P_{a}}{P_{\Omega}}=U\left(N, a, T_{I}, \Pi_{I}, W, R_{0}\right)
$$

Note that the kink safety factor $q_{*}$ and the elipticity $\kappa$ do not appear in the above equation. Specifically $q_{*}$ and $\kappa$ are considered constant and in the following results they have the values $q_{*}=2.0$ and $\kappa=2.0$. The four parameters $T_{I}, \Pi_{I}, W, R_{0}$ are determined from Eqs. $(3.24,3.25,3.40,3.54)$. In turn the quantity $P_{a} / P_{\Omega}$ is minimized in the $N, a$ space.

The first step in the solution procedure is the characterization of the parameter $\widehat{W}$. In general the parameter $\widehat{W}$ can be written as

$$
\widehat{W}=C_{1} R_{0}^{\alpha_{1}} a^{\alpha_{2}} B^{\alpha_{3}} I^{\alpha_{4}} \kappa^{\alpha_{5}}
$$

By adjusting the parameters $\alpha_{j}$ various confinement scalings can be simulated. Table 3.3 gives the values of the parameters $\alpha_{1}, \alpha_{2}, \alpha_{3}, \alpha_{4}, \alpha_{5}$ for Neo-Alcator, Goldston, Kaye Goldston, and ITER [28] confinement scalings.

Another parameter that must be given is the coil throat volume $V$. The coil volume is a constraint in the calculation and the optimization is performed subject to a fixed coil volume. In order for a baseline to be set, enabling comparisons to be made, the results that follow are obtained by taking $V=12 \mathrm{~m}^{3}$ which corresponds to the coil throat volume of the present CIT design. 

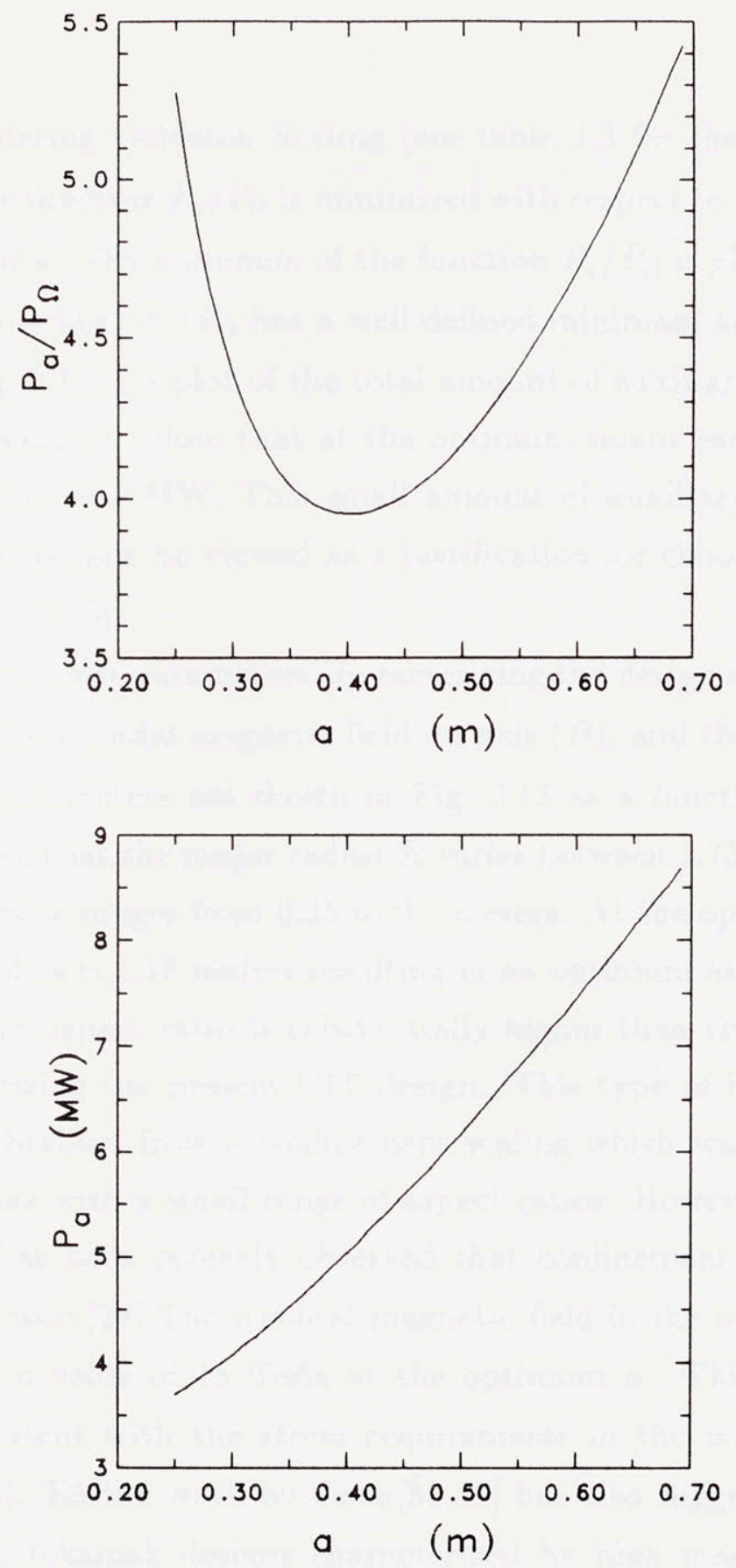

Figure 3.12: A plot of the quanity $P_{a} / P_{\Omega}$ versus the minor radius $a$, and the total auxiliary power $P_{a}$ versus $a$ 
By considering Goldston Scaling (see table 3.3 for the appropriate parameters) the function $P_{a} / P_{\Omega}$ is minimized with respect to $N$ within a range of minor radii $a$. The minimum of the function $P_{a} / P_{\Omega}$ is plotted versus $a$ in Fig. 3.12. Note that $P_{a} / P_{\Omega}$ has a well defined minimum at $a \sim 0.4 m$. Also shown in Fig. 3.12 is a plot of the total amount of auxiliary power required at ignition versus $a$. Note that at the optimum minor radius the required auxiliary power is $5 \mathrm{MW}$. This small amount of auxiliary power required for ignition may now be viewed as a justification for choozing to minimize the quantity $P_{a} / P_{\Omega}$.

Other important parameters characterizing the design are the the major radius $(R)$, the toroidal magnetic field on axis $(B)$, and the plasma current $(I)$. These parameters are shown in Fig. 3.13 as a function of the minor radius $a$. Note that the major radius $R$ varies between 1.75 and 2 meters as the minor radius ranges from 0.25 to 0.7 meters. At the optimum $a=0.4 \mathrm{~m}$ the major radius is 1.78 meters resulting in an optimum aspect ratio of 4.5 . This optimum aspect ratio is substantially higher than the aspect ratio of 3.3 characterizing the present CIT design. This type of high aspect ratio design was obtained from a confinement scaling which was derived mainly from tokamaks with a small range of aspect ratios. However, in the TFTR tokamak it has been recently observed that confinement scales favorably with aspect ratio.[29] The toroidal magnetic field in the optimized designs is high with a value of 15 Tesla at the optimum $a$. This high magnetic field is consistent with the stress requirements in the coil structure (see section 3.3.2). Earlier work by Cohn[30,31] has also suggeted the possible advantage of tokamak designs characterized by high magnetic fields. In particular Cohn's work has shown the advantage of designs characterized by high values of the parameter $B^{2} a$. Usually for $B^{2} a \geq 150$ ohmic ignition is possible thereby minimizing the adverse effect of auxiliary power on machine performance. The parameters $B \tau_{E} / R$ and $B^{2} a$ are equivalent for the case of Neo-Alcator scaling and for operation under a fixed Murakami parameter 
Table 3.4: Summary of the optimized design parameters for Goldston, NeoAlcator, and ITER scalings

\begin{tabular}{|c|c|c|c|}
\hline \hline & Goldston & Neo-Alcator & ITER \\
\hline$a(m)$ & 0.4 & 0.32 & 0.4 \\
$R(m)$ & 1.78 & 1.76 & 1.79 \\
$R / a$ & 4.5 & 5.5 & 4.47 \\
$B(T)$ & 14.5 & 16.5 & 14.8 \\
$I(M A)$ & 6.5 & 5.0 & 6.75 \\
$P_{a}(M W)$ & 5.0 & 1.2 & 2.3 \\
$T_{I}(k e V)$ & 8.5 & 5.0 & 5.7 \\
$N_{I}$ & 0.75 & 1.1 & 0.99 \\
$B \tau_{E} / R$ & 7.2 & 19.0 & 14.1 \\
\hline \hline
\end{tabular}

$n R / B$. The plasma current $I$ decreases from $11 \mathrm{MA}$ at $a=0.64 m$ to 6.5 MA at the optimum $a=0.4 \mathrm{~m}$. This decrease is a consequence of the decrease in the plasma minor radius $a$. Note that, even though the total plasma current is low at the optimum design point, the designs should not be considered low current devices since $q_{*}$ has been set at the MHD stability limit $\left(q_{*}=2\right)$.

In Fig. 3.14 the parameter $W=B \tau_{E} / R$ is plotted as a function of the plasma radius $a$. Note that the parameter $B \tau_{E} / R$ is maximized at the optimum radius $a$.

Similar results are obtained for other scalings. For the ITER scaling the optimum $P_{a} / P_{\Omega}$ and the corresponding $B \tau_{E} / R$ is plotted on Fig. 3.15. Note again thet there is a well defined miinor radius $a$ at which $P_{a} / P_{\Omega}$ is minimized. On table 3.4 the parameters representing the optimized design for Goldston, Neo-Alcator, and ITER confinement scalings are summarized.

\subsection{Conclusions}

The volume averaged plasma power balance gives the characteristics of the global plasma behavior. Since the transport of heat is governed by the 

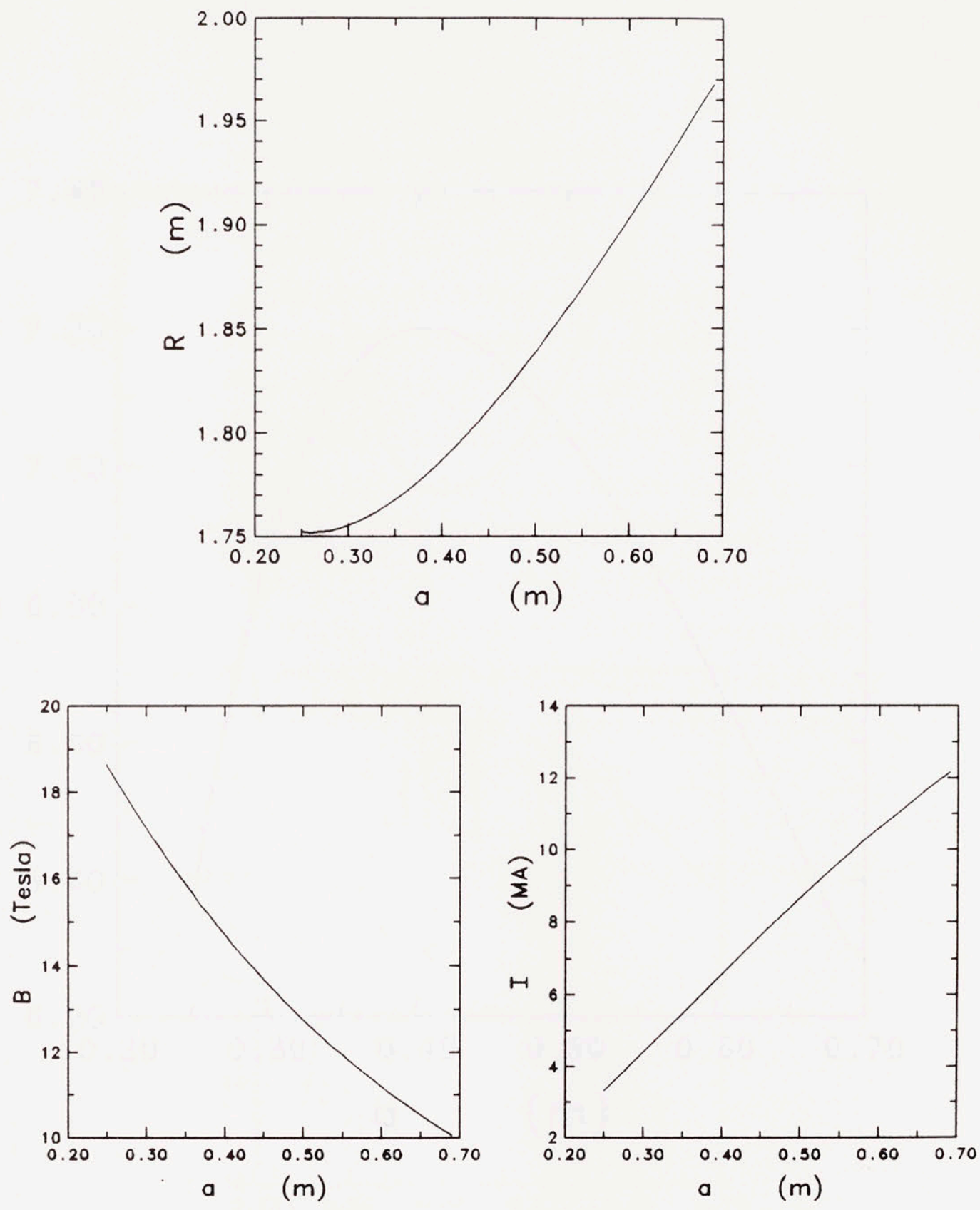

Figure 3.13: Clockwise from top are plotted the major radius $R$ the plasma current $I$ and the toroidal magnetic field $B$ as a function of radius $a$ 


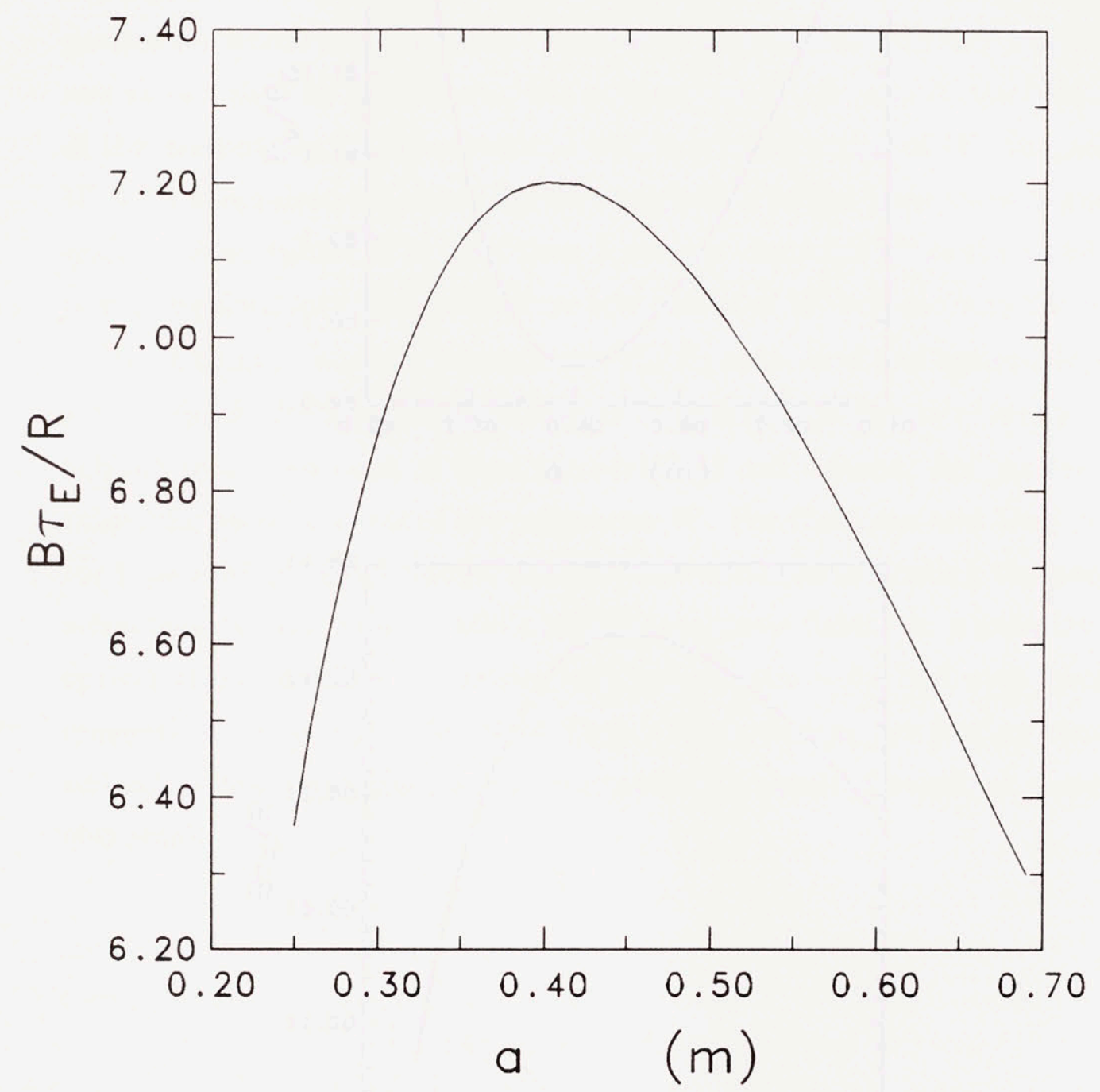

Figure 3.14: The optimized performance parameter $B \tau_{E} / R$ as a function of the plasma minor radius 

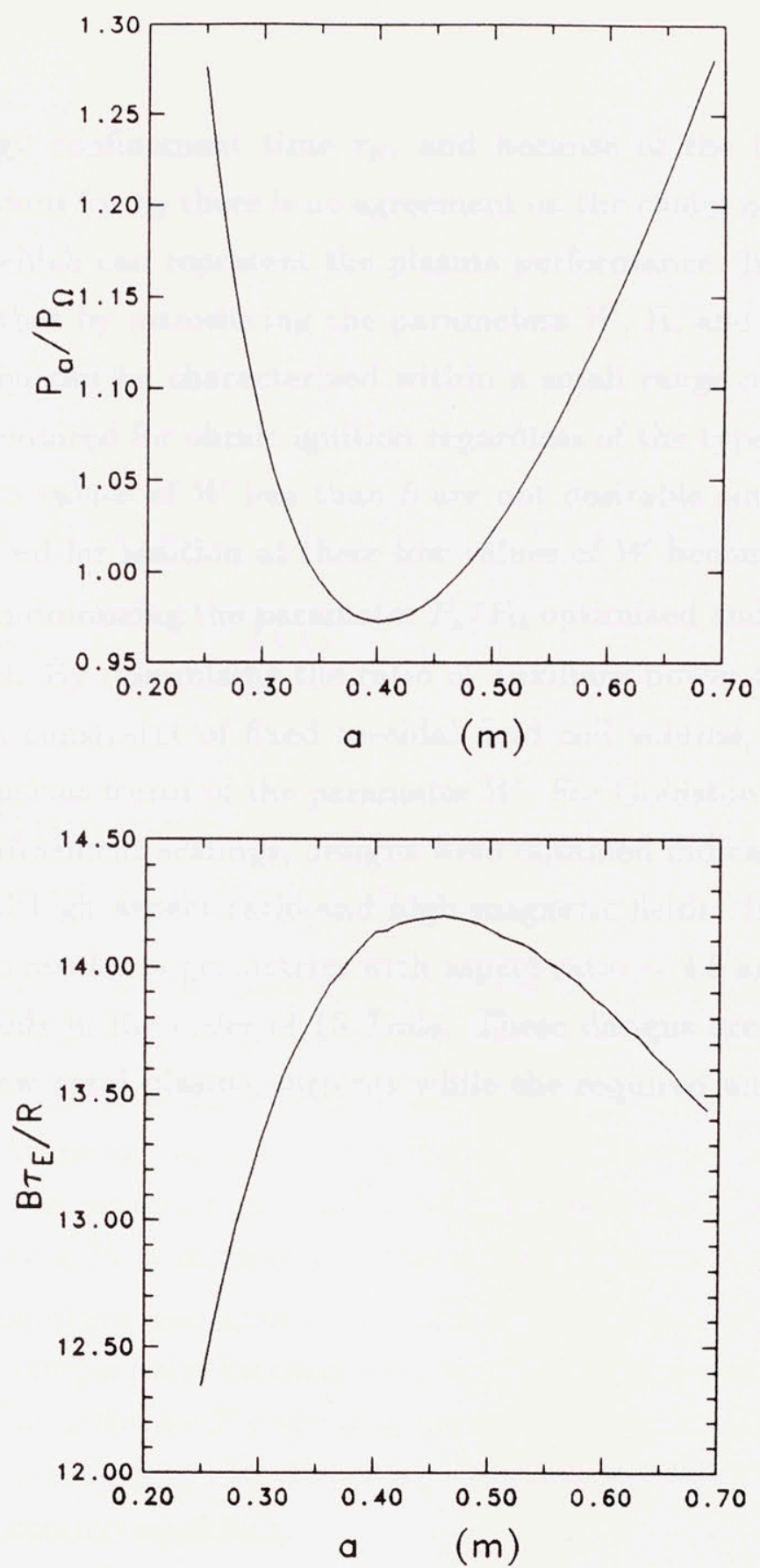

Figure 3.15: The optimized parameter $P_{a} / P_{\Omega}$ (top) and the optimized performance parameter $B \tau_{E} / R$ (bottom) as a function of the plasma minor radius corresponding to ITER scaling 
global energy confinement time $\tau_{E}$, and because of the large number of scaling relations for $\tau_{E}$ there is no agreement on the choice of an appropriate parameter which can represent the plasma performance. In this chapter it was shown that by introducing the parameters $W, \Pi$, and $N$ the behavior of the plasma can be characterized within a small range of $W$. In general $W \sim 15$ is required for ohmic ignition regardless of the type of confinement scaling. Also values of $W$ less than 5 are not desirable since the auxiliary power required for ignition at these low values of $W$ become very large.

Next by minimizing the parameter $P_{a} / P_{\Omega}$ optimized and ignited designs are obtained. By minimizing the ratio of auxiliary power to ohmic power, subject to a constraint of fixed toroidal field coil volume, designs are obtained for various forms of the parameter $W$. For Goldston and Neo- Alcator type confinement scalings, designs were obtained indicating the posible advantage of high aspect ratio and high magnetic fields. In particular the optimization results in geometries with aspect ratio $\sim 4.5$ and with toroidal magnetic fields in the order of 15 Tesla. These designs are further characterized by low total plasma currents while the required auxiliary power is also small. 


\section{Chapter 4}

\section{Burn Control Using Auxiliary Power Modulation}

\subsection{Tokamak Thermal Instability and the Need for Burn Control}

Thermal runaway of a tokamak plasma occurs once the plasma temperature exceeds a certain value. Fig. 4.1 shows the rate of change of temperature, $\dot{T}$, as a function of temperature, $T$ for CIT (Table 4.1) under Goldston $\mathrm{H}$-mode confinement. As is shown in Fig. 4.2 auxiliary power $P_{I}$ is required in order for the plasma temperature to enter into the ignition regime. If the temperature $T_{I}$ is reached and the auxiliary power is not reduced the plasma temperature continues to increase since $\dot{T}>0$ in this region.

It is this temperature increase that we call thermal runaway.

By examining the $n-T$ operating space of CIT under Goldston H-mode confinement scaling (see Fig. 4.3) it is possible to distinguish the regions of stable and unstable equilibria.

By expanding the energy balance Eq. (2.229) about the equilibria shown in Fig. 4.3 the linear growth rate $\gamma\left(\mathrm{sec}^{-1}\right)$ is calculated and its values are shown in Fig. 4.4 as linear growth rate contours. A positive sign for $\gamma$ indicates the unstable region, while a negative sign implies stable equilibria.

Note that there are two regions of stable equilibria. One at low temperatures and the other at high temperatures. The low temperature stable 


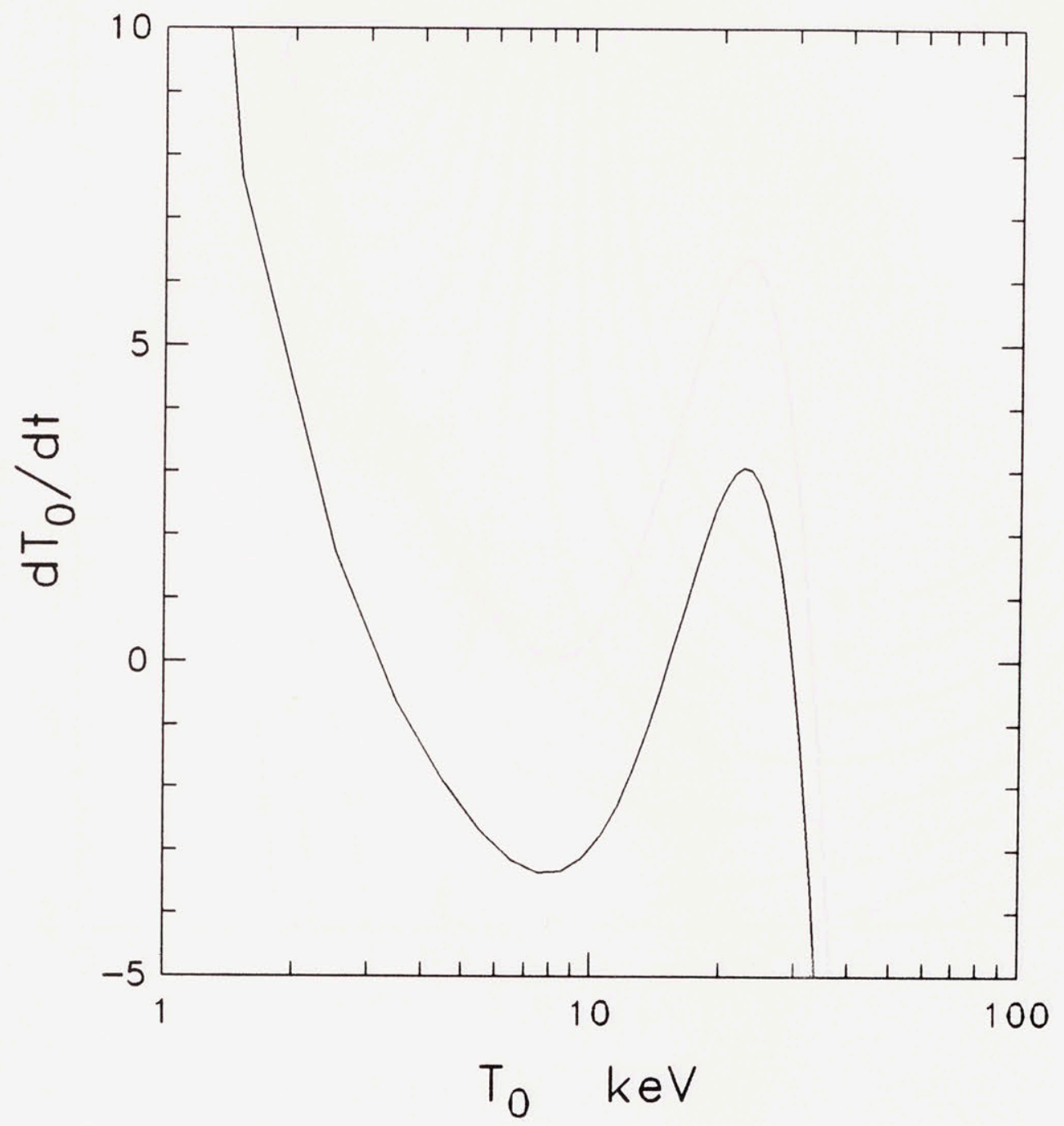

Figure 4.1: CIT $\dot{T}$ vs $T$ plot for the parameters shown on Table 4.1 and for zero auxiliary power. 


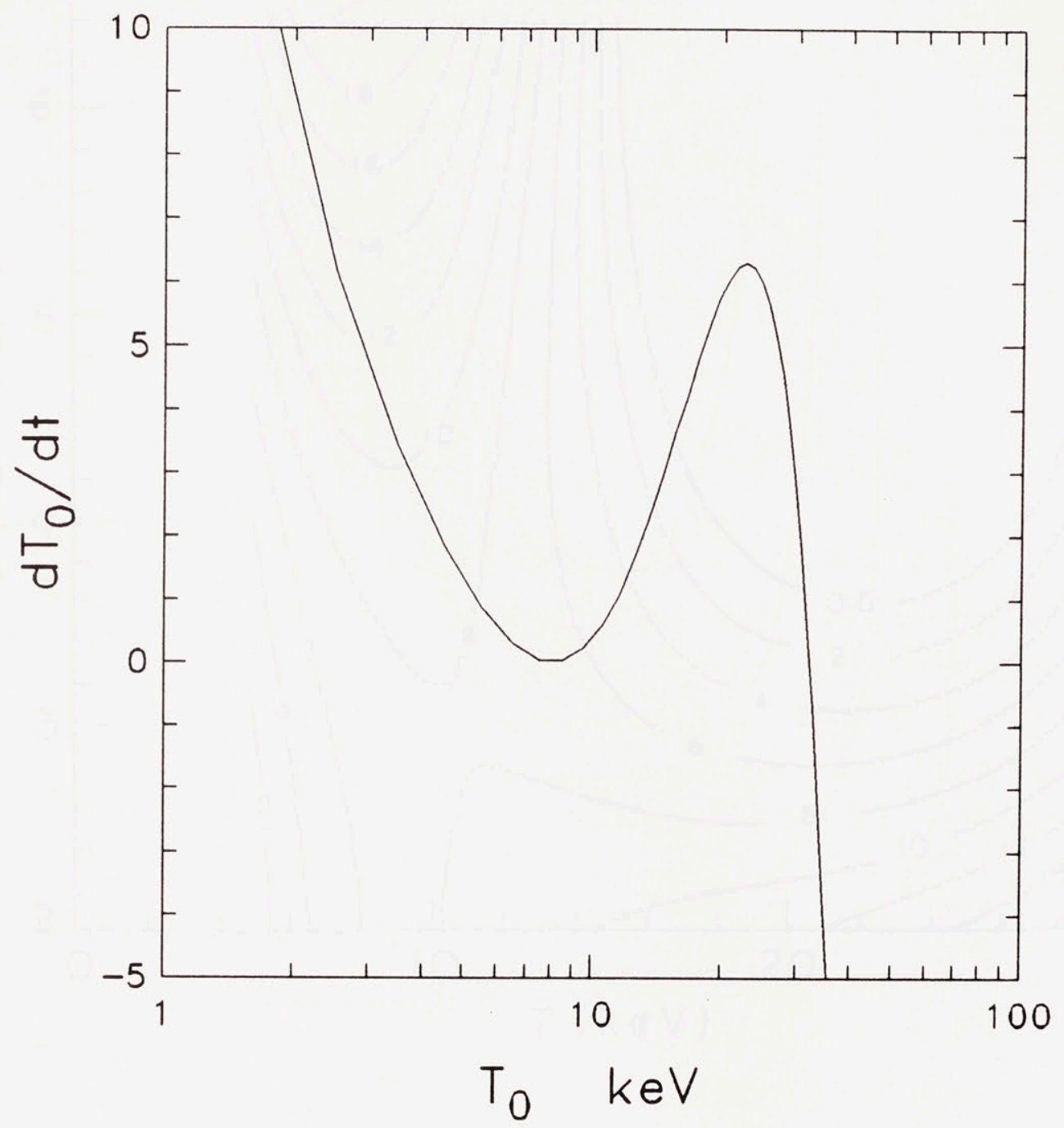

Figure 4.2: CIT $\dot{T}$ vs $T$ plot for the parameters shown on Table 4.1 and for $18 \mathrm{MW}$ of auxiliary power. 


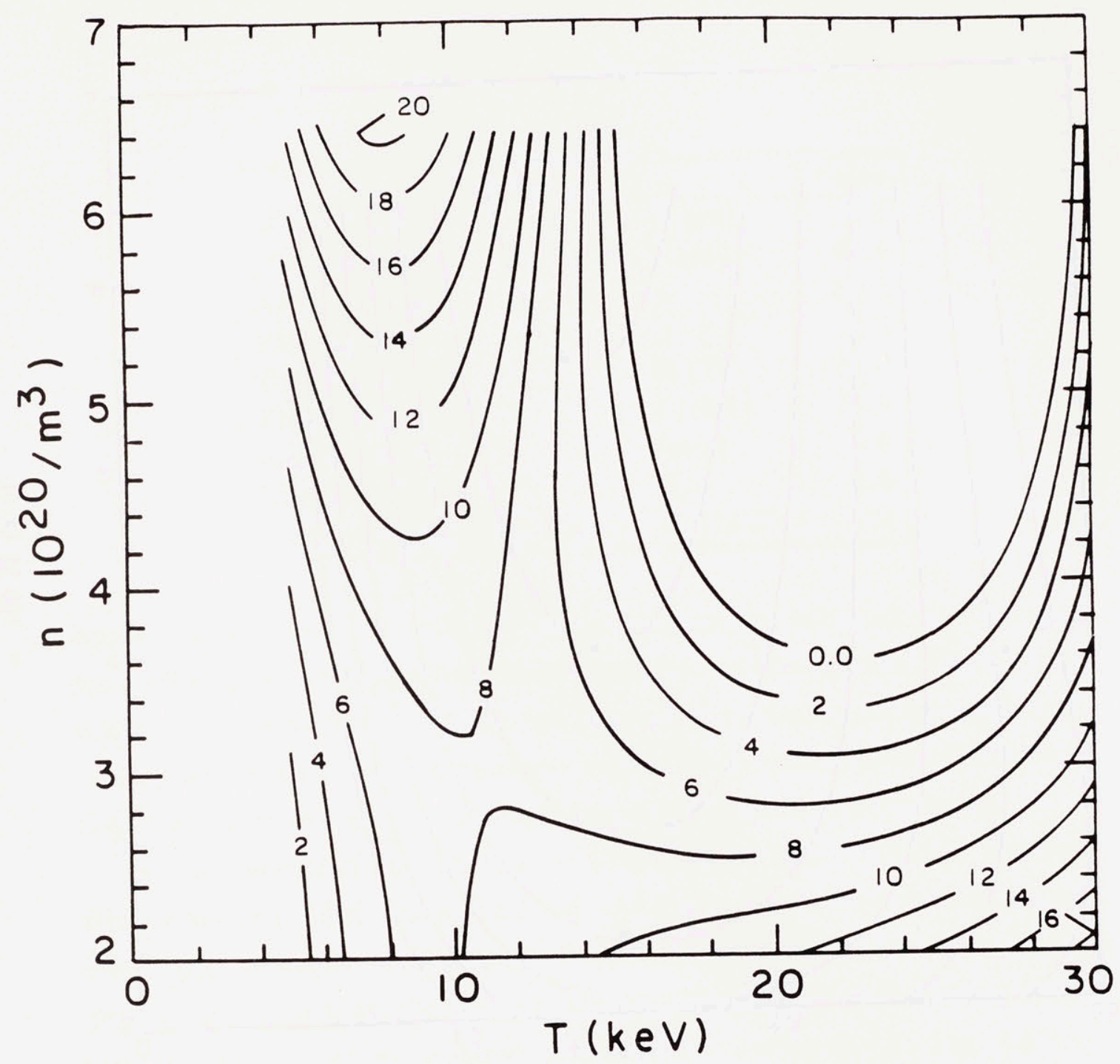

Figure 4.3: CIT plasma operating contours of auxiliary power for Goldston $\mathrm{H}$-mode scaling 


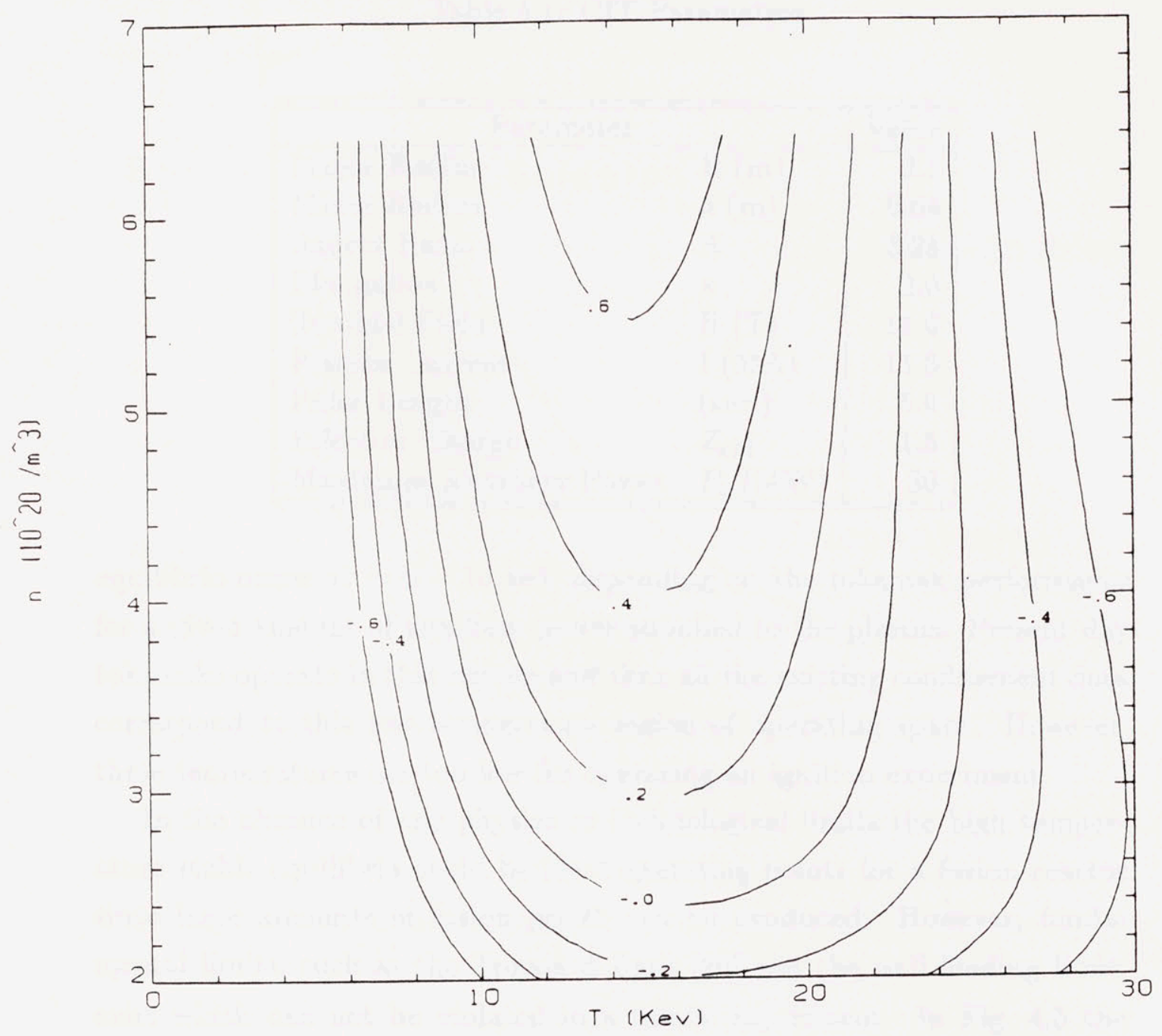

Figure 4.4: n-T plot with contours of linear growth rates $(\gamma)$ of plasma temperature corresponding to Fig. 4.3 
Table 4.1: CIT Parameters

\begin{tabular}{|ll|r|}
\hline \hline \multicolumn{2}{|c|}{ Parameter } & Value \\
\hline Major Radius & $\mathrm{R}(\mathrm{m})$ & 2.1 \\
Minor Radius & $\mathrm{a}(\mathrm{m})$ & 0.64 \\
Aspect Ratio & $\mathrm{A}$ & 3.28 \\
Elongation & $\kappa$ & 2.0 \\
Toroidal Field & $\mathrm{B}(\mathrm{T})$ & 11.0 \\
Plasma Current & $\mathrm{I}(\mathrm{MA})$ & 11.0 \\
Pulse Length & $(\mathrm{sec})$. & 5.0 \\
Effective Charge & $Z_{\text {eff }}$ & 1.5 \\
Maximum Auxiliary Power & $P_{a}(\mathrm{MW})$ & 30 \\
\hline \hline
\end{tabular}

equilibria occur at $\sim 5-10 \mathrm{keV}$ depending on the tokamak performance for a given amount of auxiliary power supplied to the plasma. Present day tokamaks operate in this regime and thus all the existing confinement data correspond to this low temperature region of operating space. However, these temperatures are too low for operating an ignition experiment.

In the absence of any physics or technological limits the high temperature stable equilibria could be ideal operating points for a fusion reactor since large amounts of fusion power can be produced. However, fundamental limits, such as the Troyon $\beta$ limit [20] and the wall loading limit, exist which can not be violated in a fusion experiment. In Fig. 4.5 the CIT equilibria corresponding to zero auxiliary power are shown under NeoAlcator,[13] Goldston-H,[14] and Kaye-Goldston-H [15] scalings.

Note that the location of the high temperature stable equilibria (dashed lines) can vary between 20 and $80 \mathrm{keV}$ depending on the type of confinement assumed. In general, an ignited plasma will evolve to one of these high temperature equilibria. Also on Fig. 4.5 typical contours for the $\beta$ limit and the wall loading limit of $3 \mathrm{MW} / \mathrm{m}^{2}$ are shown. These limits are violated for some of these equilibria. It is plausible to speculate that the expected uncertainty in the location of these high temperature equilibria will be as 


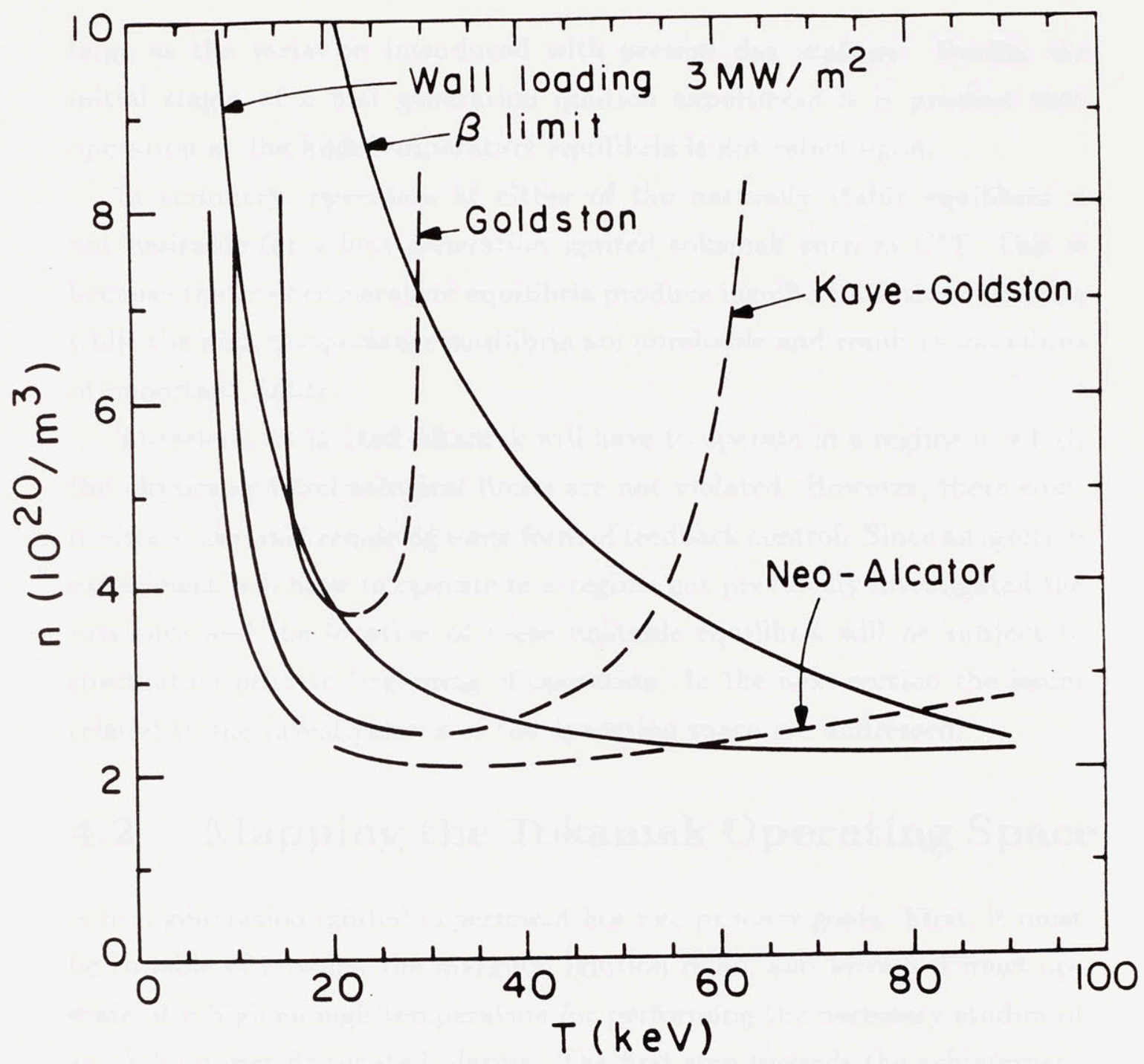

Figure 4.5: Location of the high temperature stable equilibria corresponding to zero auxiliary power under various confinement scalings. 
large as the variation introduced with present day scalings. During the initial stages of a first generation ignition experiment it is prudent that operation at the high temperature equilibria is not relied upon.

In summary, operation at either of the naturally stable equilibria is not desirable for a first generation ignited tokamak such as CIT. This is because the low temperature equilibria produce insufficient fusion reactions while the high temperature equilibria are unreliable and result in violations of important limits.

Therefore, an ignited tokamak will have to operate in a regime in which the physics and technological limits are not violated. However, these equilibria are unstable requiring some form of feedback control. Since an ignition experiment will have to operate in a regime not previously investigated the existence and the location of these unstable equilibria will be subject to speculation prior to beginning of operation. In the next section the issues related to the investigations of the operating space are addressed.

\subsection{Mapping the Tokamak Operating Space}

A first generation ignited experiment has two primary goals. First, it must be capable of crossing the marginal ignition ridge, and second it must operate at a high enough temperature for performing the necessary studies of an alpha power dominated plasma. The first step towards the achievement of these goals is the determination of the existence of a regime inside which an ignited plasma can operate.

Due to the uncertainty associated with the predictions of existing confinement scalings the characteristics of the operating space in an ignited tokamak are not accurately known. For example, in a steady state POPCON plot with fixed auxiliary power contours the precise locations of the marginal ignition ridge, and the zero auxiliary power contour are unknown. Therefore, determining the characteristics of the operating space, (mapping the operating space) will be the first goal of the ignition experiment. In this 
section various methods by which the operating space can be mapped are investigated.

During the mapping stage of an ignition experiment the most important goal is to determine the existence and location of the marginal ignition ridge. If such region exists the plasma is capable of igniting according to the definition given in chapter 3 , i.e. the alpha power will dominate other terms in the energy balance equation. Knowing the location of this ridge is important since it separates the low temperature stable region from the high temperature unstable region. A successful experiment must achieve some form of "stabilized" operation in the high temperature region.

Auxiliary power, whose primary objective is to assist the plasma in crossing the marginal ignition ridge, may be used for mapping the tokamak operating space both in the low temperature stable regime and in the high temperature ignition regime. The primary motivation for using auxiliary power is that it is capable of creating steady state equilibria at any location, provided that sufficient power is available. For a fixed plasma density a certain auxiliary power $P_{I}$ is required to enter the ignition regime. For $P_{a}<P_{I}$ an equilibrium point if reached to the left of the MIR. If $P_{a}>P_{I}$ the plasma temperature will continue to increase, resulting in a thermal runaway, unless the power is appropriately reduced. The procedure by which the auxiliary power is changed is outlined in section 4.5.

In an experiment, the location of the marginal ignition ridge can be determined by either (1) measuring the energy confinement time and extrapolating (2) measuring Q (Fusion power / Auxiliary power) as a function of temperature, (3) looking for an inflection point in the temperature versus time plot. In this analysis we assume that acceptable reproducibility exists from shot to shot, and thus we assume that once the behavior at a certain point is known it will remain the same as long as the operating conditions do not change.

The energy confinement time may be measured during the temperature evolution or more easily during a temperature flat-top. By using auxiliary 
power, as outlined above, temperature equilibria can be created. By measuring the peak temperature and density as well as the profile shapes during the flat-top the energy confinement time $\tau_{E}$ is essentially deduced from the volume average of the equation

$$
P_{a}=\frac{n T}{\tau_{E}}+P_{b}-\frac{P_{\text {fusion }}}{5}-P_{\Omega}
$$

The fusion power may be deduced from the density and temperature measurements or directly from appropriately calibrated neutron detectors. The amount of auxiliary power absorbed by the plasma is estimated by modeling the energy transfer mechanism of the particular form of auxiliary power used.

Once the fusion power produced by the plasma $P_{f}$ and the auxiliary power $P_{A}$ absorbed by the plasma are known the value of $Q=P_{f} / P_{a}$ is estimated. For a given density the value of $Q$ at the marginal ignition ridge is found by simultaneously solving the equations

$$
\begin{aligned}
\frac{d T}{d t} & =0 \\
\frac{d P_{a}}{d T} & =0
\end{aligned}
$$

In Fig. 4.6 the value of $Q$ at the marginal ignition ridge of CIT is plotted as a function of plasma density $n$ under various confinement scalings. Note that $Q$ ranges from 4 to 6 as the density varies from its value at the Cordey pass to the Murakami limit.

It is therefore obvious that a $Q$ of at least 5 must be achieved before any claim for an ignited plasma can be made. Therefore, once $Q=5$ is detected the implication is that the plasma is capable of igniting. The importance of the $Q=5$ contour is also shown in Figs. 4.7, 4.8, and 4.9 where the marginal ignition ridge (MIR) and the $Q=5$ contour are shown for Neo-Alcator, 


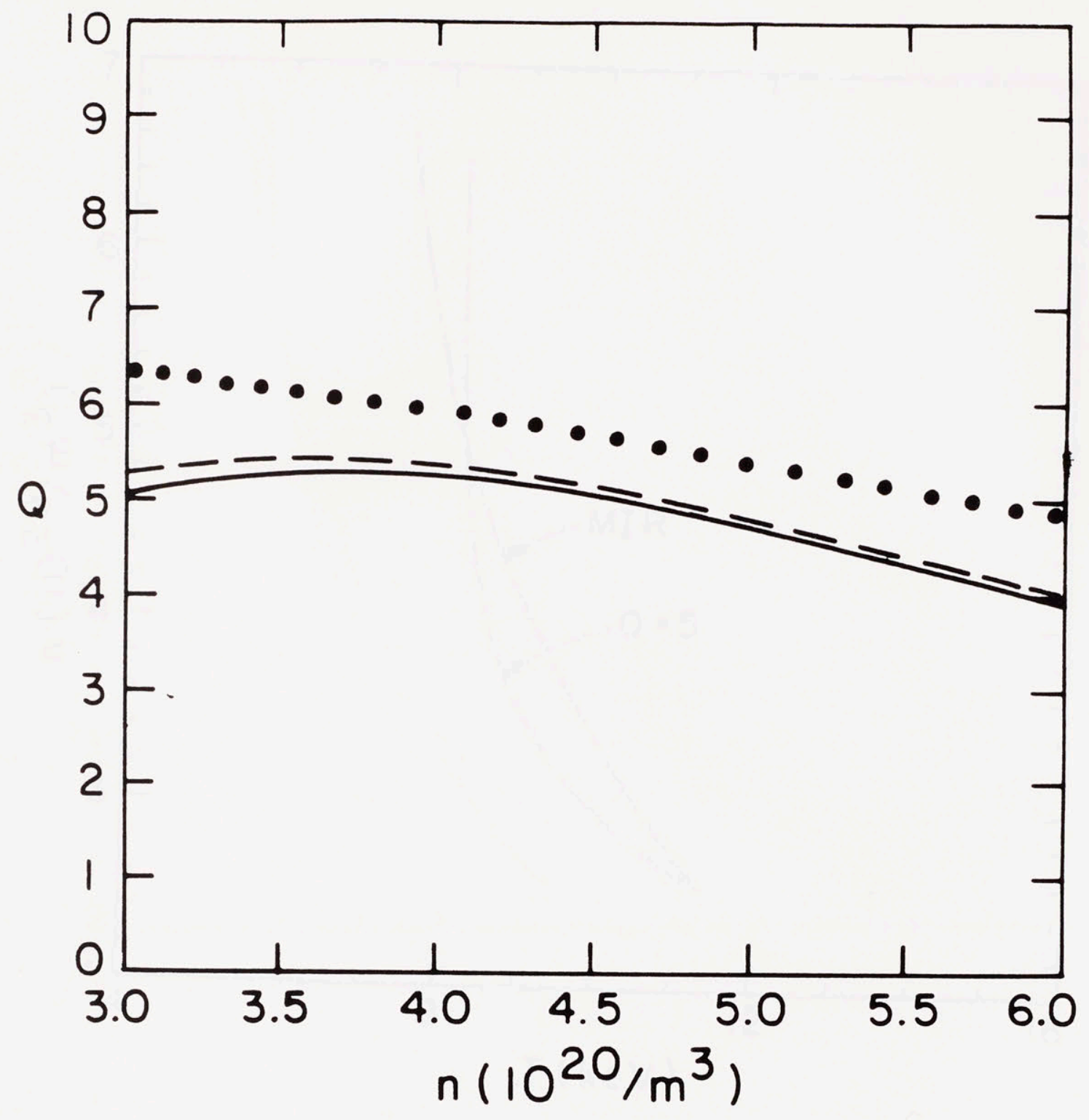

Figure 4.6: $Q$ at the marginal ignition ridge (MIR) as a function of plasma density for Neo-Alcator (solid line), Goldston (dotted line), and KayeGoldston (dashed line) confinement scalings 


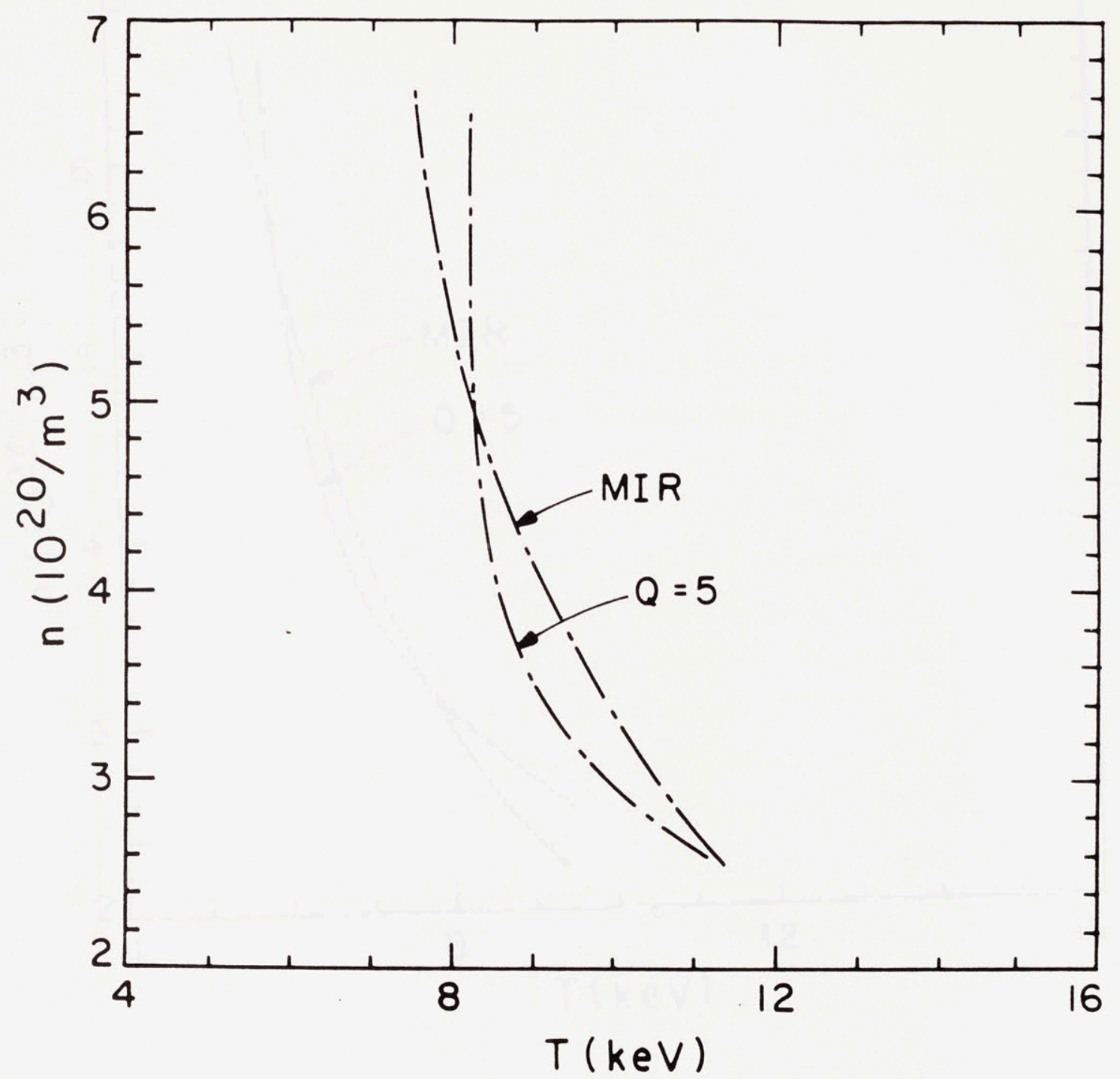

Figure 4.7: The marginal ignition ridge (MIR) and the $Q=5$ contour are shown for CIT under Neo-Alcator scaling 


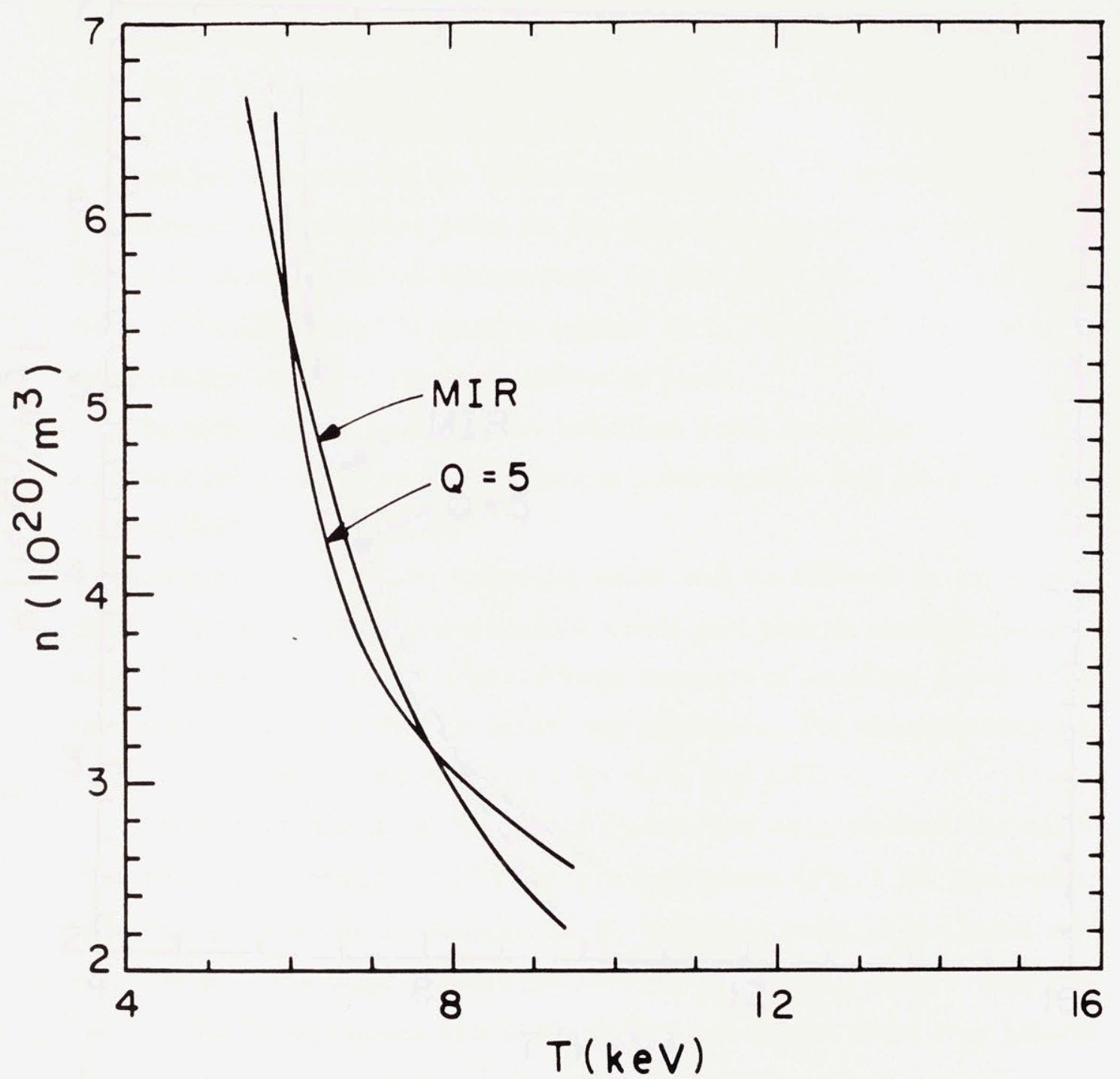

Figure 4.8: The marginal ignition ridge (MIR) and the $Q=5$ contour are shown for CIT under Goldston H-mode scaling 


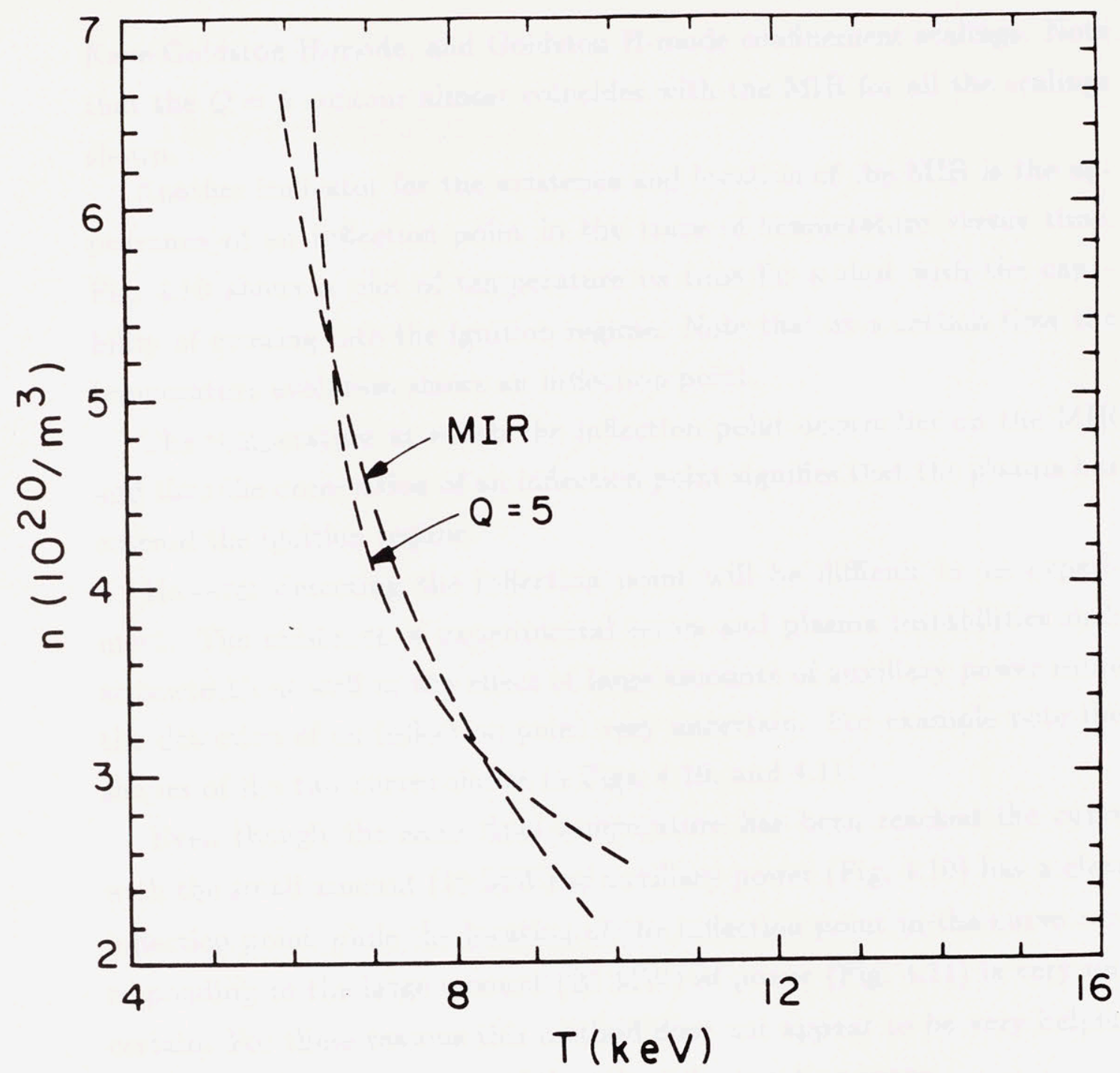

Figure 4.9: The marginal ignition ridge (MIR) and the $Q=5$ contour are shown for CIT under Kaye-Goldston H-mode scaling 
Kaye-Goldston H-mode, and Goldston H-mode confinement scalings. Note that the $Q=5$ contour almost coincides with the MIR for all the scalings shown.

Another indicator for the existence and location of the MIR is the appearance of an inflection point in the trace of temperature versus time. Fig. 4.10 shows a plot of temperature $v s$ time for a shot with the capability of crossing into the ignition regime. Note that at a certain time the temperature evolution shows an inflection point.

The temperature at which the inflection point occurs lies on the MIR and thus the observation of an inflection point signifies that the plasma has entered the ignition regime.

However detecting the inflection point will be difficult in an experiment. The existence of experimental errors and plasma instabilities such as sawteeth as well as the effect of large amounts of auxiliary power make the detection of an inflection point very uncertain. For example note the shapes of the two curves shown in Figs. 4.10, and 4.11.

Even though the same final temperature has been reached the curve with the small amount (10 MW) of auxiliary power (Fig. 4.10) has a clear inflection point while the location of the inflection point in the curve corresponding to the large amount (20 MW) of power (Fig. 4.11) is very uncertain. For these reasons this method does not appear to be very helpful during the mapping process of the tokamak operating space.

\subsection{Burn Control Issues}

The goal of burn control is to provide a stable thermal equilibrium in the regime of alpha dominated ignited operation. In general, any viable burn control study requires consideration of the following issues: determination of the equilibrium "operating" temperature, stability analysis about the operating temperature, calculation of the time scales involved in the problem, and evaluation of the various diagnostic and engineering issues. 


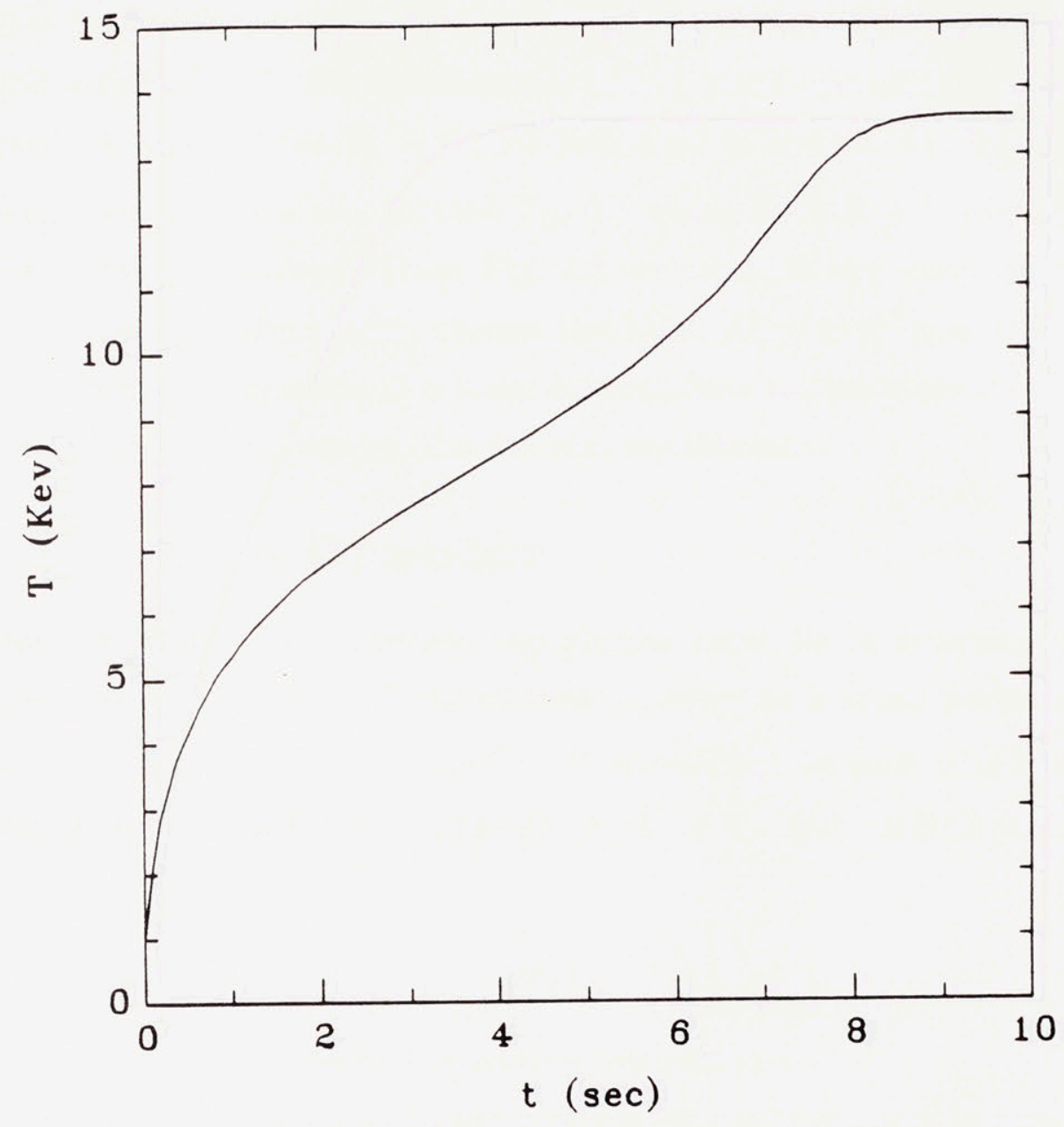

Figure 4.10: Temperature evolution of an ignited CIT with "small" amount of auxiliary power. 


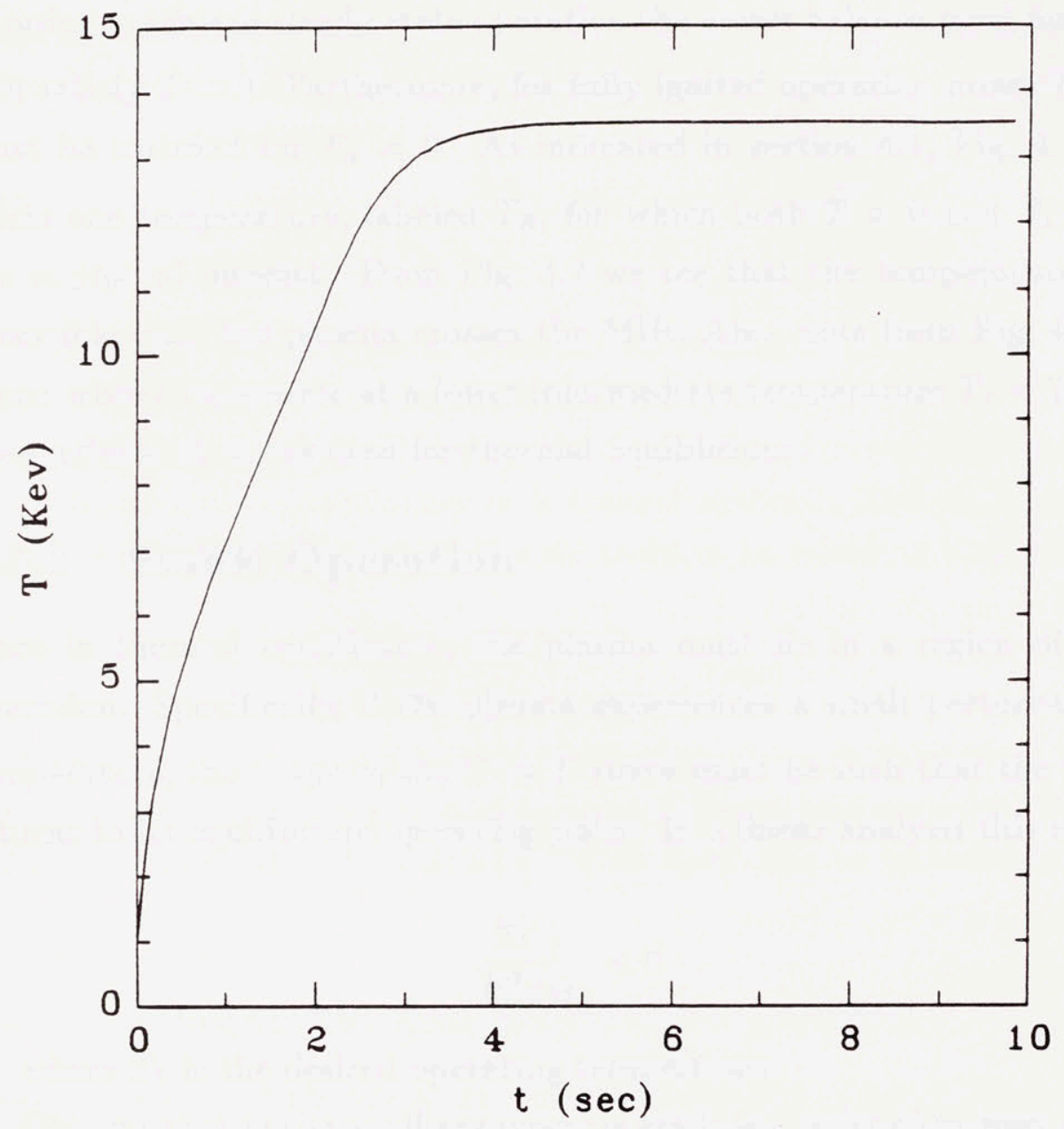

Figure 4.11: Temperature evolution of an ignited CIT with "large" amount of auxiliary power. 


\subsubsection{Thermal Equilibrium}

In order to achieve steady state operation the power balance must by definition satisfy $\dot{T}=0$. Furthermore, for fully ignited operation power balance must be satisfied for $P_{a}=0$. As indicated in section 4.1, Fig. 4.1 there exists one temperature, labeled $T_{B}$, for which both $\dot{T}=0$ and $P_{a}=0$ in the regime of interest. From Fig. 4.2 we see that the temperature $T_{B}$ is accessible once the plasma crosses the MIR. Also, note from Fig. 4.2 that if one wishes to operate at a lower intermediate temperature $T_{I}<T<T_{B}$, then a finite $P_{a}$ is required for thermal equilibrium.

\subsubsection{Stable Operation}

Once in thermal equilibrium, the plasma must lie in a region of stable operation. Specifically, if the plasma experiences a small perturbation in temperature, the shape of the $\dot{T}$ vs $T$ curve must be such that the plasma returns to its equilibrium operating point. In a linear analysis this requires

$$
\left.\frac{\partial \dot{T}}{\partial T_{S}}\right|_{T_{S}}<0
$$

where $T_{S}$ is the desired operating temperature.

Observe that as the auxiliary power is gradually reduced to zero, the operating temperature approaches $T_{B}$ (see Fig. 4.1). At $T_{B}$ Eq. 4.4 is not satisfied and thus $T_{B}$ is an unstable equilibrium. Providing a realistic method for stabilizing operation about $T \approx T_{B}$ is the primary goal of burn control studies.

\subsubsection{Time Scales}

A critical feature of any burn control system is its response time. In the case of an active control scheme, there may be a substantial delay between the time that the temperature of the plasma is detected and the time that the 
system responds by altering the plasma temperature for stability. For effective stabilization against temperature excursions, the burn control system must be able to respond on a time scale shorter than the thermal runaway time $\tau_{R}$. In general, the e-folding thermal runaway time $\tau_{R}$ is given by

$$
\tau_{R}=\frac{3 n}{\left.\frac{d}{d T}\left(P_{\alpha}+P_{\Omega}+P_{a}-P_{l}-P_{b}\right)\right|_{T_{S}}}=\left(\left.\frac{\partial \dot{T}}{\partial T}\right|_{T_{S}}\right)^{-1}
$$

Depending on the type of confinement scaling used $\tau_{R}$ varies between 0.5 and 2.0 seconds in the regime of interest.

The delay time associated with the deposition of power into the plasma is an important consideration in a control system. The alpha particles deliver most of their energy to the electrons in an e-folding time given by

$$
\tau_{\alpha e}=\frac{0.099}{\ln \Lambda} \frac{T^{3 / 2}}{n} \quad(\mathrm{sec})
$$

Similar estimates can be made for the time delay in the energy transfer between the auxiliary power mechanism and the plasma particles. The time delay associated with the feedback system must also be included as well as detection delays.

\subsubsection{Diagnostic and Engineering Issues}

Burn control methods based on active stabilization of temperature excursions depend on monitoring the plasma parameters. Therefore, the overall response of the feedback control system is determined by both the inaccuracies of the diagnostics and the finite delays inherent in the system. Deviations from the stable equilibrium temperature cause changes in the fusion power production that introduce damaging cyclic heat and neutron fluxes to the first wall and supportive structures. Therefore, it is important to keep temperature excursions to a minimum. This requires accurate temperature measurements and fast response of the feedback system.

A successful burn control system must satisfy each of the conceptual requirements described above as well as a large number of detailed practical problems. 


\subsection{Burn Control Methods}

Over the years researchers have suggested a variety of methods for providing temperature control to a burning plasma. In this section a review of the most widely studied burn control methods is presented. The various methods are compared by briefly explaining the physical principle and outlining the major advantages and disadvantages of each. The primary motivation for the selection of a particular burn control method is reliability, both technological and scientific. While a number of methods may actually be tested in an ignited fusion experiment, it is clearly important that the baseline design must be based on the ones with the most reliability in order to insure overall credibility. The various control mechanisms may be classified as passive or active depending on whether the modifications to the energy transport in the plasma is achieved by the plasma itself (passive) or by actively altering the plasma operating conditions.

\subsubsection{Passive Methods}

\subsubsection{Soft $\beta$ Limit Control}

It has been suggested that the plasma may automatically provide itself with burn control by means of a soft $\beta$ limit. $[32,33,34]$ In particular, as the plasma undergoes a thermal runaway, it will eventually exceed the Troyon $\beta$ limit. To the extent that this is a "soft" limit, the consequence is a rapid deterioration of confinement time, resulting in automatic burn control. Economically and technologically such a method is highly desirable. However, the existence and behavior of a soft $\beta$ limit is not well established. Thus, the method does not have high scientific reliability. Furthermore, if the $\beta$ limit is hard rather than soft, the result is a major disruption, leading to potentially serious damage to the first wall. Furthermore, the $\beta$ limit must also be less than the wall loading limit, particularly in a reactor.

\subsubsection{Field Ripple Control}

Another method of natural burn control relies on the prediction, from neoclassical transport theory, that toroidal field ripple can significantly enhance 
ion heat transport. $[35,36,37,38,39]$ To achieve control, the magnetic field is designed so that the ripple increases strongly with major radius near the outside of the torus. Thus, as the plasma heats during a thermal runaway, its Shafranov shift increases, moving the plasma into a region of strong ripple. This provides automatic (natural) burn control. Again the primary advantages of such a method are its economic and technological attractiveness. The problems with ripple control are as follows. First, the enhanced transport resulting from field ripple has not been reliably verified experimentally. Second, if the plasma ignites in an optimized region of minimum field ripple, it is not possible to stabilize against negative thermal excursions. Finally, if one needs to stabilize relatively large positive temperature deviations, the field ripple must be actively increased over a short time scale. However, due to the long time scale associated with magnetic field diffusion, this may be impossible to accomplish.

\subsubsection{Active Methods}

\subsubsection{Vary $B_{0}$.}

If the toroidal magnetic field $B_{0}$ is varied above and below the value which gives the desired equilibrium temperature, then for essentially all confinement scalings the performance parameter $n \tau$ varies accordingly. This in principle should be an effective way to stabilize both positive and negative temperature excursions. The major difficulty with this procedure is that the magnetic diffusion time $\tau_{D} \approx \mu_{0} a^{2} / \eta$, where $a$ and $\eta$ are respectively the radius and resistivity of the plasma, is very long, thus prohibiting effective stabilization of fast temperature excursions. Also, such a control system requires that the value of the steady state toroidal magnetic field be below the design limit in order to insure that negative temperature deviations can be stabilized. Furthermore, the power requirements for varying the magnetic field may be prohibitively large. 


\subsubsection{Adiabatic Expansion / Compression}

By varying the vertical field during a thermal runaway, the plasma can expand (or compress) adiabaticlly. [40,41,42,43] For an expansion, the resulting state has lower $n$, lower $T$, larger $R$ and larger $a$. In most circumstances the strong $n, T$ dependence of the alpha particle heating dominates. This cools the plasma, thereby providing a mechanism for burn control. The difficulties with this method involve power requirements on the vertical field circuit, and the need for a somewhat oversized vacuum chamber to allow room for expansion and contraction. The problem is more serious in configurations with divertors, since there is in general only very limited flexibility in the allowable motion of the $X$ points of the separatrix.

\subsubsection{Vary the Plasma Density $n_{0}$}

If the density can be varied above and below its thermal equilibrium value, the resulting change in alpha particle power should be adequate to provide burn control. There are several important difficulties with this method. Since the density diffusion time scale is typically three to five times longer than the energy confinement time, a combination of gas puffing and particle diffusion does not have adequate response time to stabilize the thermal runaway. Part of the difficulty can be overcome by injecting hydrogen pellets into the plasma. The increased mass cools the plasma but does not produce any additional alpha particles. This process takes place on a very short time scale, more than adequate for burn control. Thus, pellet injection can effectively stabilize positive thermal excursions. Negative temperature excursions are more difficult to control. Injecting pellets of $\mathrm{D}$ or $\mathrm{T}$ should in principle increase the plasma reactivity. However, once a pellet is injected, the immediate plasma response is a drop in $\mathrm{T}$ and an increase in $\mathrm{n}$, further amplifying the initial negative temperature excursion. Whether or not the temperature can recover quickly enough to reheat the plasma is uncertain. 


\subsubsection{Vary the Auxiliary Power $P_{a}$}

Since a certain auxiliary power is required for ignition, lowering $P_{a}$ sufficiently fast during the thermal runaway can cause a net loss in plasma energy; the decrease in plasma energy due to lower $P_{a}$ exceeds the increase due to the thermal runaway in $P_{\alpha}$. $[44,45,46]$ This procedure can stabilize a positive thermal excursion. Conversely, rapidly increasing $P_{a}$ during a negative temperature excursion, also produces stability. Auxiliary heating in the form of $\mathrm{RF}$ power can be varied in a relatively continuous manner on a virtually instantaneous time scale. In this regard, varying $P_{a}$ is an effective way to achieve burn control. The primary disadvantage is that in order to stabilize both positive and negative temperature excursions, the value of $P_{a}$ must be non-zero at the point of thermal equilibrium. Therefore, the overall efficiency of the reactor, defined by $Q \equiv$ Fusion power $\left(P_{f}\right) /$ Auxiliary power $\left(P_{a}\right)$, can be unacceptably low if the maximum temperature excursion to be stabilized is too large (requiring a large equilibrium $P_{a}$ ).

From the discussion above, it is apparent that there are a wide variety of possible burn control methods. Each has advantages and disadvantages, and none is sufficiently simple and well tested experimentally to assure success. Passive burn control methods such as soft the $\beta$ limit control or field ripple are more desirable from an economic point of view. It is therefore important that future fusion experiments carry out studies aimed at investigating the validity of such methods. However, in judiciously designing near term experiments such as CIT and ITER the primary burn control method must utilize the most reliable method available.

Burn control by means of varying $P_{a}$ is the best choice for the first generation ignition experiments. Significant auxiliary power already exists for ignition, so no additional major hardware is required. Varying $P_{a}$ provides stability against both positive and negative temperature excursions. The main drawback is that in steady state the plasma must operate sub-ignited requiring a finite non-zero value of $P_{a}$. However, we show in the analysis that follows, that the $Q$ of the system (fusion power over the auxiliary power) remains quite high for reasonable values of the thermal excursion $\Delta T$ that must be stabilized. For these reasons, the remainder of the analysis is focussed on burn control by means of varying $P_{a}$. 


\subsection{Auxiliary Power Control: General Re- marks}

Burn control with auxiliary power modulation is obtained by varying the input power as a function of plasma parameters such as temperature or fusion power (neutron flux). At the beginning of the discharge the maximum amount of auxiliary power is supplied to the plasma. Once the temperature crosses the MIR the plasma enters into the unstable ignition regime. Once in this regime the auxiliary power will be appropriately reduced in order to create an equilibrium at the desired temperature. Quantitatively the auxiliary power term in the energy balance, (Eq. 2.229) is varied according to the proportional feedback control law:

$$
P_{a}(T)=\left\{\begin{array}{cl}
P_{I}+\Delta P & T<T_{1} \\
\left(P_{I}+\Delta P\right)\left[1-\left(\frac{T-T_{1}}{T_{2}-T_{1}}\right)^{\lambda}\right] & T_{1}<T<T_{2} \\
0 & T>T_{2}
\end{array}\right.
$$

where $P_{I}$ is the minimum auxiliary power required to cross the MIR, $\Delta P$ is excess power above this value required for a finite time evolution to the desired operating point, $T_{1}$ is a temperature $\left(\sim T_{I}\right)$ above which the auxiliary power is gradually reduced, and $T_{2}$ is a temperature $\left(\sim T_{B}\right)$ between $T_{1}$ and the burn temperature $T_{B}$ at which $P_{a}=0$. The exponent $\lambda$ dictates the shape of the $P_{a}$ versus $T$ curve.

By changing the parameters in Eq. 4.7 a steady state operating temperature can be created somewhere between $T_{1}$ and $T_{B}$. Figure 4.12 illustrates (a) the original system, (b) the form of the applied auxiliary power, and (c) the final system with steady state operating temperature $T_{S}$.

Initially, auxiliary power equal to $P_{I}+\Delta P$ is supplied to the plasma. By doing so the plasma temperature increases and eventually the MIR temperature $T_{I}$ is reached. The temperature continues to increase until the value 

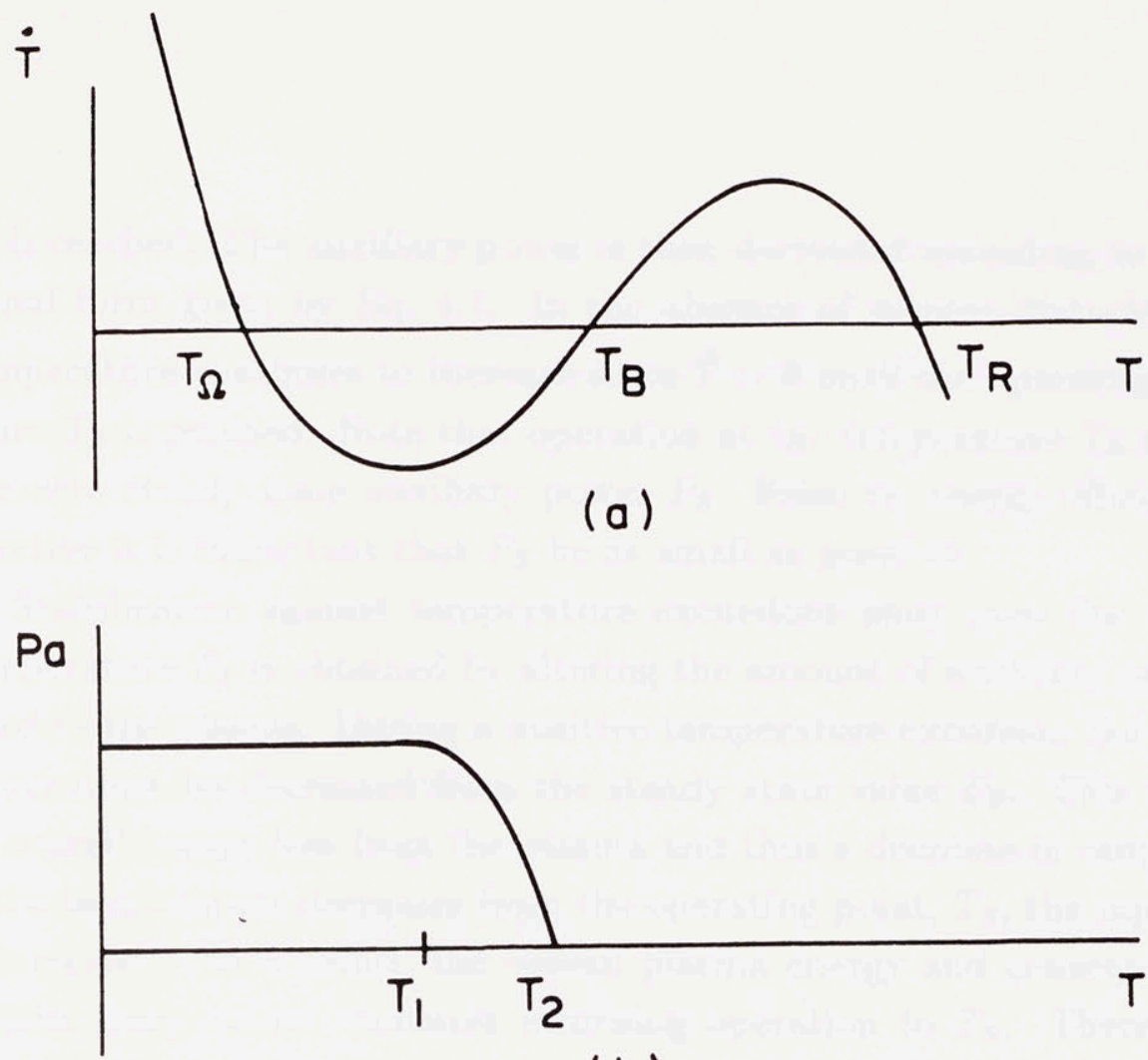

(b)

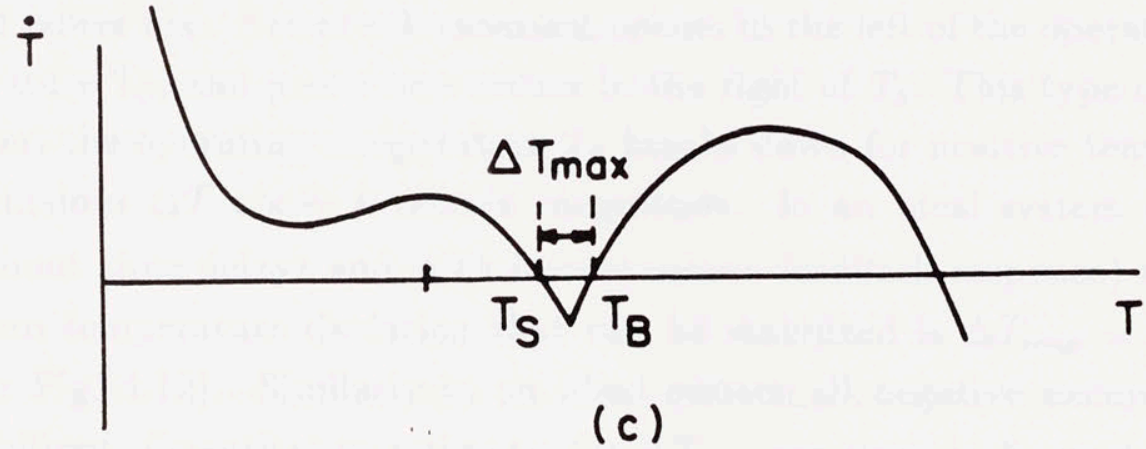

Figure 4.12: Schematic representation of an original system (a), the form of the auxiliary power (b), and the final system indicating the steady state operating temperature $T_{S}$ 
$T_{1}$ is reached. The auxiliary power is then decreased according to the functional form given by Eq. 4.7. In the absence of plasma disturbances the temperature continues to increase since $\dot{T}>0$ until the operating temperature $T_{S}$ is reached. Note that operation at the temperature $T_{S}$ requires a non-zero steady state auxiliary power $P_{S}$. From an energy efficiency perspective it is important that $P_{S}$ be as small as possible.

Stabilization against temperature excursions away from the operating temperature $T_{S}$ is obtained by altering the amount of auxiliary power supplied to the plasma. During a positive temperature excursion the auxiliary power must be decreased from the steady state value $P_{S}$. This results in an overall energy loss from the plasma and thus a decrease in temperature. If the temperature decreases from the operating point, $T_{S}$, the input power is increased. As a result, the overall plasma energy and consequently the plasma temperature increases returning operation to $T_{S}$. Therefore, the auxiliary power should be viewed as a source of both power enhancement and power loss. Power enhancement occurs to the left of the operating temperature $T_{S}$, and power loss occurs to the right of $T_{S}$. This type of control about the operating temperature $T_{S}$ breaks down for positive temperature excursions $\Delta T$ above a certain magnitude. In an ideal system (i.e. one without time delays and with instantaneous feedback response) the maximum temperature deviation that can be stabilized is $\Delta T_{\max }=T_{B}-T_{S}$ (see Fig. 4.12). Similarly in an ideal system all negative excursions are stabilized. Quantitative estimates for $\Delta T_{\max }$ are given in figure 4.21.

\subsection{The Complete Burn Control Model}

In addition to the power balance Eq. (2.222), a complete burn control model must include the effects of the time delays discussed in section 4.3 , the form of the feedback law given in section 4.5 as well as the density evolution.

\subsubsection{Time Delays}

In this model the time delay associated with the energy transfer between the fusion alpha particles and the plasma ions as well as the time delay 
associated with the deposition of auxiliary power into the plasma is considered.

In general, the effect at the present time $t$ of a stimulus $S_{1}\left(t^{\prime}\right) d t^{\prime}$ at any past time $t^{\prime}$ is given by

$$
S_{2}(t)=\int_{0}^{t} \mathcal{R}\left(t-t^{\prime}\right) S_{1}\left(t^{\prime}\right) d t^{\prime}
$$

where it is assumed that the effect is proportional to the stimulus with proportionality constant $\mathcal{R}$ which depends only on the elapsed time $t-t^{\prime}$. Hence it has the form $\mathcal{R}\left(t-t^{\prime}\right)$. Eq. (4.8) gives the response at the present time $t$ as a weighted superposition over the input at the times $t^{\prime}<t$. The weighting factor $\mathcal{R}\left(t-t^{\prime}\right)$ characterizes the system, and $S_{1}\left(t^{\prime}\right)$ characterizes the past history of the input.

In particular, since we are concerned with the delay time associated with the transfer of energy as particles collide, the delay time or the energy transfer time $\tau$ is given by

$$
\tau=-\frac{E}{d E / d t}
$$

where $E$ is the particle energy. By integrating Eq. (4.9) the rate $\mathcal{R}$ becomes

$$
\mathcal{R}\left(t_{1}\right)=-\frac{1}{E_{0}} \frac{d E\left(t_{1}\right)}{d t_{1}}=\frac{1}{\tau} \exp \left[-\frac{t_{1}}{\tau}\right]
$$

In Eq. (4.10) $t_{1}=t-t^{\prime}$ and represents the time elapsed since the particle had energy $E_{0}$. By substituting Eq. (4.10) into Eq. (4.8) and differentiating with respect to $t$ we obtain

$$
\frac{d E_{2}(t)}{d t}=\frac{1}{\tau}\left[E_{1}(t)-E_{2}(t)\right]
$$

This is now a differential equation giving the effect of the stimulus, in this case the amount of energy transferred into the plasma, at time $t$ subject 
to the delay time $\tau$ and some appropriate initial condition. (i.e. $E_{1}$ is the energy produced by the particles at time $t$, and $E_{2}$ is the energy absorbed by the plasma at time $t$ )

Similarly the time response of the feedback system, including the diagnostics and the auxiliary power delivery system, can be modeled by evaluating the auxiliary power at a delayed temperature $T_{d}$. A simple model for the feedback system is obtained by assuming that the delayed temperature $T_{d}$ relaxes to the plasma temperature with a time constant $\tau_{d}[45]$

$$
\frac{d T_{d}}{d t}=\frac{1}{\tau_{d}}\left(T-T_{d}\right)
$$

\subsubsection{Complete Auxiliary Power Control Model}

The energy balance, Eq. (2.229), the time delays associated with the alpha power, Eq. (4.11) and the feedback system, Eq. (4.12) and equation (2.232) describing evolution of plasma density are coupled to obtain the following system of ordinary differential equations.

$$
\begin{aligned}
\frac{d T}{d t} & =\mathcal{G}\left(T, n_{e}, n_{i}, P_{\alpha}\left(n_{d}, n_{t}, T\right), P_{\alpha}\left(T_{d}\right)\right) \\
\frac{d P_{\alpha}}{d t} & =\frac{1}{\tau_{\alpha}}\left[Q_{\alpha}\left(n_{d}, n_{t}, T\right)-P_{\alpha}\left(n_{d}, n_{t}, T\right)\right] \\
\frac{d T_{d}}{d t} & =\frac{1}{\tau_{d}}\left[T-T_{d}\right] \\
\frac{d n_{d}}{d t} & =\mathcal{J}\left(T, n_{d}, n_{t}, S_{d}\right) \\
\frac{d n_{t}}{d t} & =\mathcal{K}\left(T, n_{d}, n_{t}, S_{t}\right) \\
\frac{d n_{\alpha}}{d t} & =\mathcal{L}\left(T, n_{d}, n_{t}\right)
\end{aligned}
$$


where the form of the functionals $\mathcal{G}, \mathcal{J}, \mathcal{K}$, and $\mathcal{L}$ are given by Eqs. $(2.229$, 2.232). $P_{a}\left(T_{d}\right)$ is given by Eq. 4.7. $P_{\alpha}(T)$ is the alpha power absorbed by the plasma at time $t, Q_{\alpha}$ represents the amount of alpha power produced at time $t$. The parameters $\tau_{\alpha}$, and $\tau_{d}$ represent the delay times associated with the alpha power, and the feedback system.

In the next section the behavior of an auxiliary power control system are investigated by solving Eqs. (4.13).

\subsection{CIT Burn Control Using Auxiliary Power Modulation}

The Compact Ignition Tokamak (CIT), whose parameters are given in table 4.1 , is designed to achieve ignition (i.e. alpha particle dominated operation) in order to determine the effectiveness of alpha particle heating and its impact on confinement.

The results are presented in two parts. First, the heating sequence is examined and the time required to reach the desired operating temperature is compared to the pulse length. In the second part operation about the equilibrium temperature is considered and the auxiliary power control system is evaluated.

\subsubsection{Temperature Evolution}

During an ignition sequence in CIT the plasma temperature must evolve, from $T=0$ at the beginning of the discharge, to the desired operating temperature, $T_{S}$, in a time significantly shorter than the specified pulse length. This is a significant problem in CIT because of the short pulse length, and thus the shot must evolve in such a way so that the time to reach the temperature $T_{S}$ is minimized. This requires that the maximum amount of auxiliary power is supplied to the plasma and that the density evolves in such a way so that the plasma evolves through the Cordey pass. In this analysis the plasma temperature evolves from zero at the beginning 
of the current flat-top to the desired temperature some time during the flat- top. The maximum amount of auxiliary power available is $30 \mathrm{MW}$ (note that this is the power coupled to the plasma). For a conservative estimate we assume that a maximum of $25 \mathrm{MW}$ can be coupled to the plasma. The results shown here, unless otherwise indicated are based on Goldston H-mode confinement.

Fig. 4.7.1 shows a CIT POP-CON plot under Goldston H-mode scaling. In this space the final operating point is denoted by point $A$ which corresponds to density of $6 \times 10^{20} / \mathrm{m}^{3}$ and a temperature of $15 \mathrm{keV}$.

Note, from Fig 4.7.1, that the Cordey pass occurs at a density of $\sim$ $3.0 \times 10^{20} / \mathrm{m}^{3}$ and a temperature of $\sim 11 \mathrm{keV}$. Here the density is varied with temperature as follows. For low temperatures and until the Cordey pass temperature is reached the density is constant and equal to $3 \times 10^{20} \mathrm{~m}^{3}$. For higher temperatures the density is ramped linearly between the Cordey pass temperature and the final operating temperature. The auxiliary power supplied to the plasma must be modulated if steady state operation is desired at $15 \mathrm{keV}$. From Fig. 4.7.1 we see that for steady state operation at a density of $6.0 \times 10^{20} / \mathrm{m}^{3}$ and a temperature of $15 \mathrm{keV}, 0.7 \mathrm{MW}$ of auxiliary power is required. Thus the power must be reduced from its maximum of $25 \mathrm{MW}$ to its steady state value of $0.7 \mathrm{MW}$ as the operating point is approached.

The plasma temperature, density, and the alpha, ohmic, auxiliary, radiation, and conduction powers are shown in Figs. 4.14, 4.15, and 4.16 for $\lambda=2, T_{1}=11 \mathrm{keV}$, and $T_{2}=16 \mathrm{keV}$. Under these conditions the plasma evolves to the final temperature in 2 seconds. The remaining 3 seconds during the current flat-top is used for physics and engineering operation at the high temperature equilibrium.

In order for the plasma density to evolve as shown in Fig. 4.15 the fueling conditions shown in Fig. 4.17 must be provided. The result shown in Fig. 4.17 were obtained by assuming that the plasma evolves through a sequence of quasistatic equilibria and thus the $d n / d t$ term was neglected in 


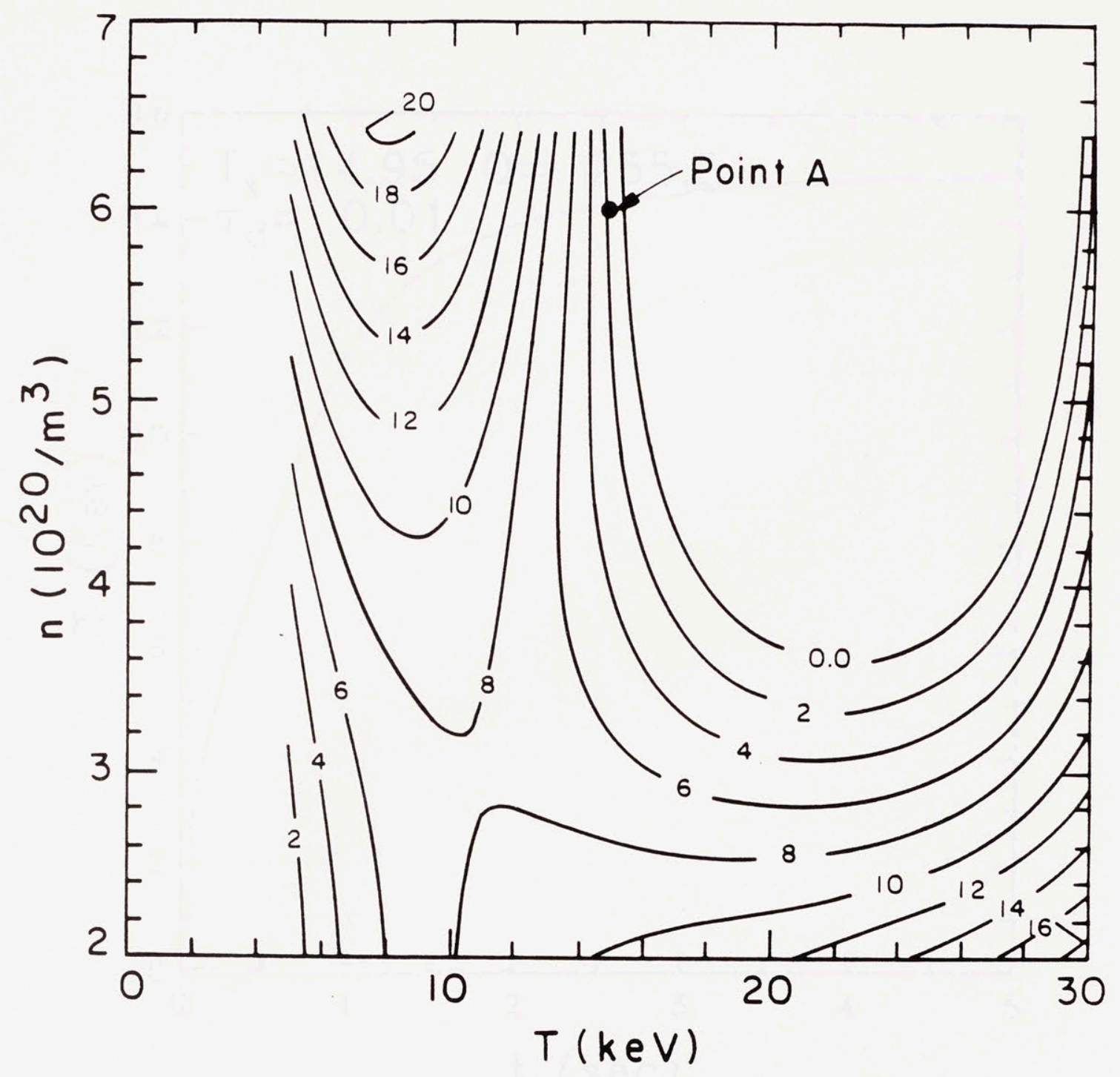

Figure 4.13: CIT POP-CON under Goldston H-mode scaling showing the desired final operating point (point A). 


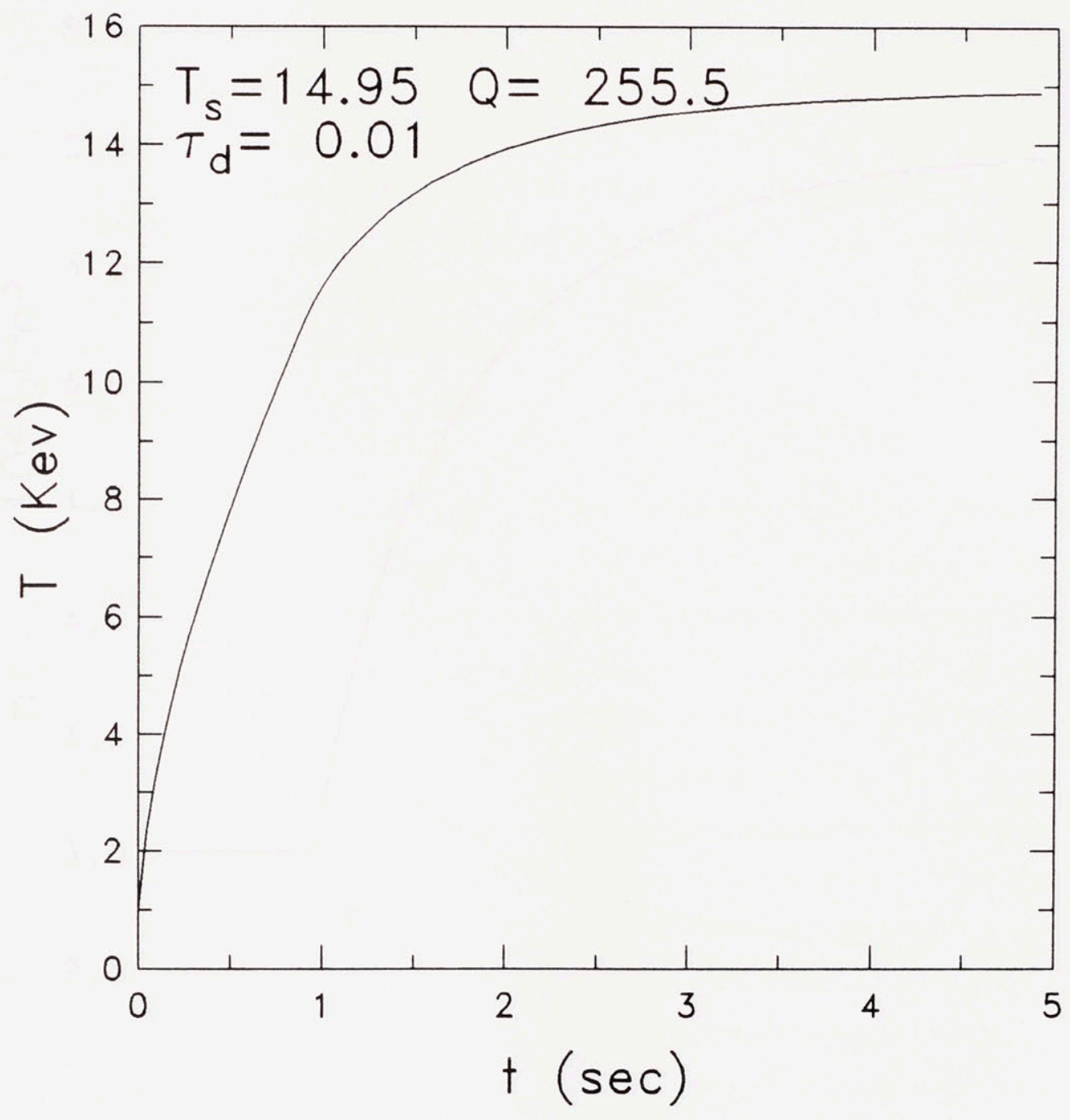

Figure 4.14: Time evolution of temperature for CIT under Goldston Hmode confinement. 


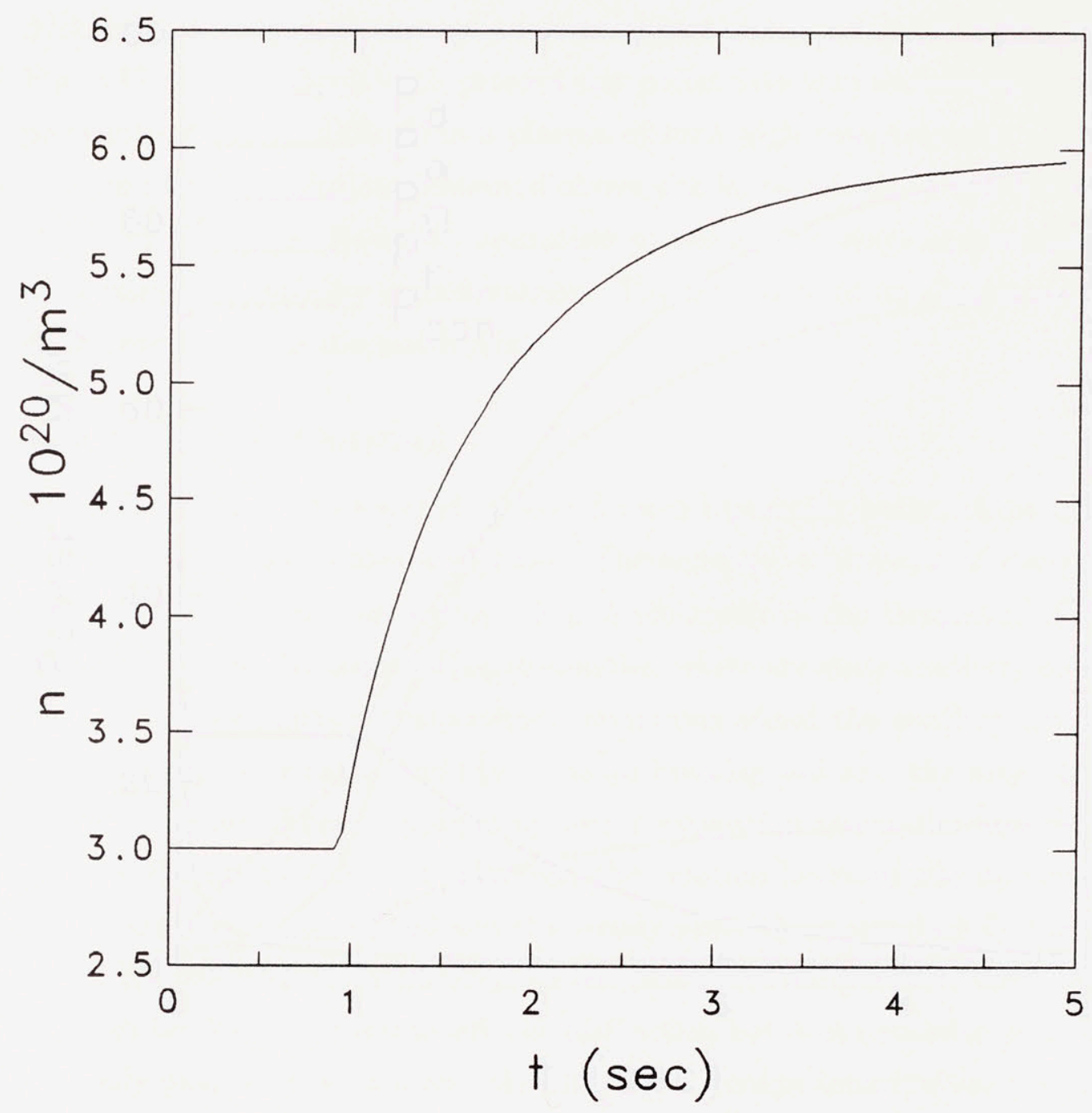

Figure 4.15: Time evolution of density for CIT under Goldston H-mode confinement. 


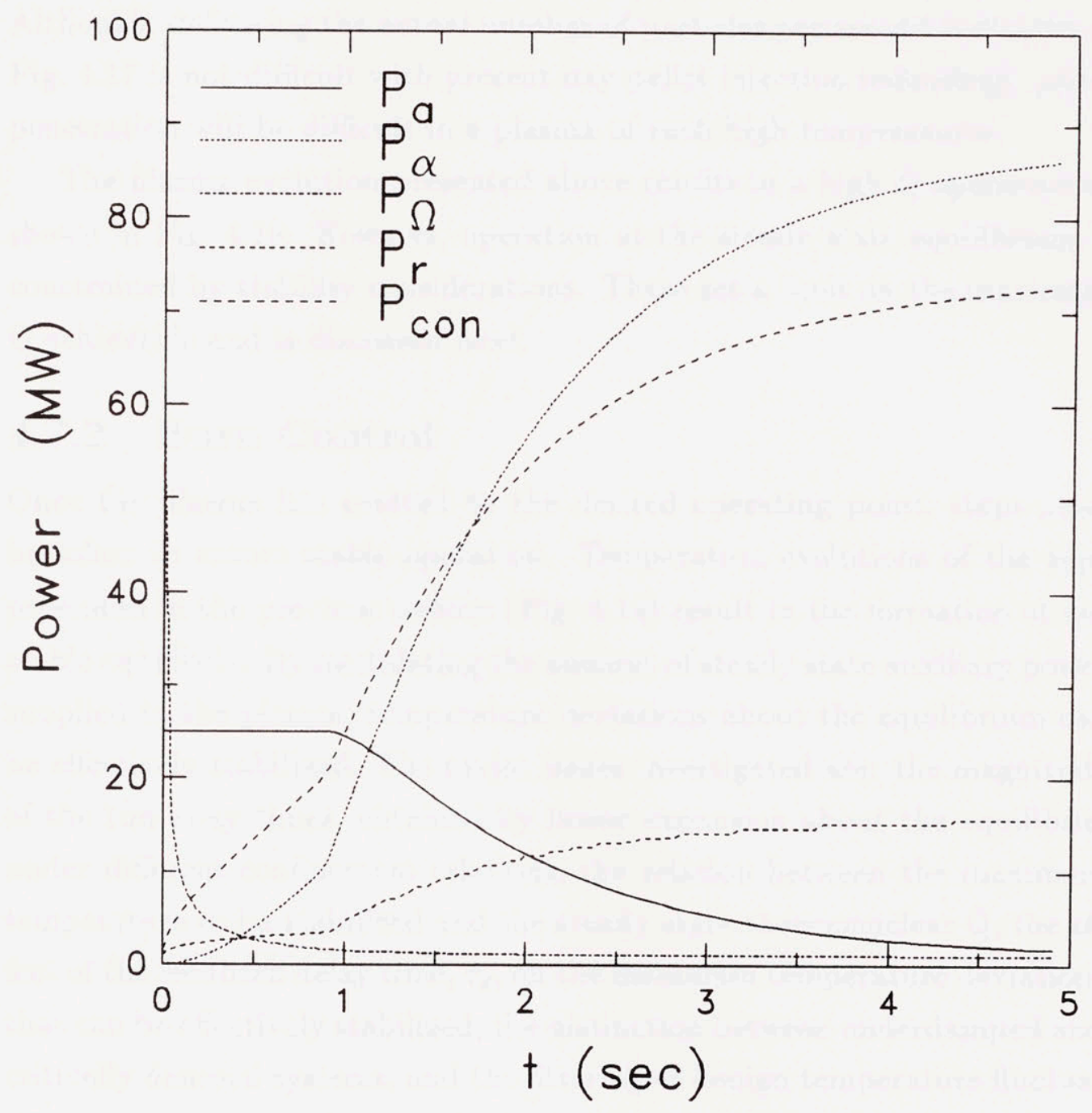

Figure 4.16: Time evolution of plasma powers for CIT under Goldston Hmode confinement. 
the calculation of the source rate $S$. As seen from Fig. 4.17 strong fueling is required if the plasma is to evolve according to the above assumptions. Although, delivering the actual number of particles per second indicated in Fig. 4.17 is not difficult with present day pellet injection technology, pellet penetration will be difficult in a plasma of such high temperatures.

The plasma evolution presented above results in a high $\mathrm{Q}$ operation as shown in Fig. 4.18. However, operation at the steady state equilibrium is constrained by stability considerations. These set a limit on the maximum $\mathrm{Q}$ achievable and is discussed next.

\subsubsection{Burn Control}

Once the plasma has evolved to the desired operating point, steps must be taken to ensure stable operation. Temperature evolutions of the type presented in the previous section (Fig. 4.14) result in the formation of unstable equilibria. By modulating the amount of steady state auxiliary power supplied to the plasma, temperature deviations about the equilibrium can be effectively stabilized. The major issues investigated are: the magnitude of the run-away times, obtained by linear expansion about the equilibria, under different confinement relations, the relation between the maximum temperature to be stabilized and the steady state thermonuclear $Q$, the effect of the feedback delay time, $\tau_{d}$, on the maximum temperature deviations that can be effectively stabilized, the distinction between underdamped and critically damped systems, and the filtering of benign temperature fluctuations, such as sawteeth, from the feedback system.

For effective stabilization of perturbations about an equilibrium temperature the feedback system must respond in a time faster than the runaway time $\tau_{R}$ of the instability. In Fig. 4.19 the e-folding time is shown as the runaway time for CIT under Goldston H-mode confinement scaling. Comparison of Fig. 4.19 with Fig. 4.20, which corresponds to Neo-Alcator scaling, shows the strong dependance of $\tau_{R}$ on confinement scalings. For example, at a density of $6 \times 10^{20} / \mathrm{m}^{3}$ and a temperature of $10 \mathrm{keV}$ the 


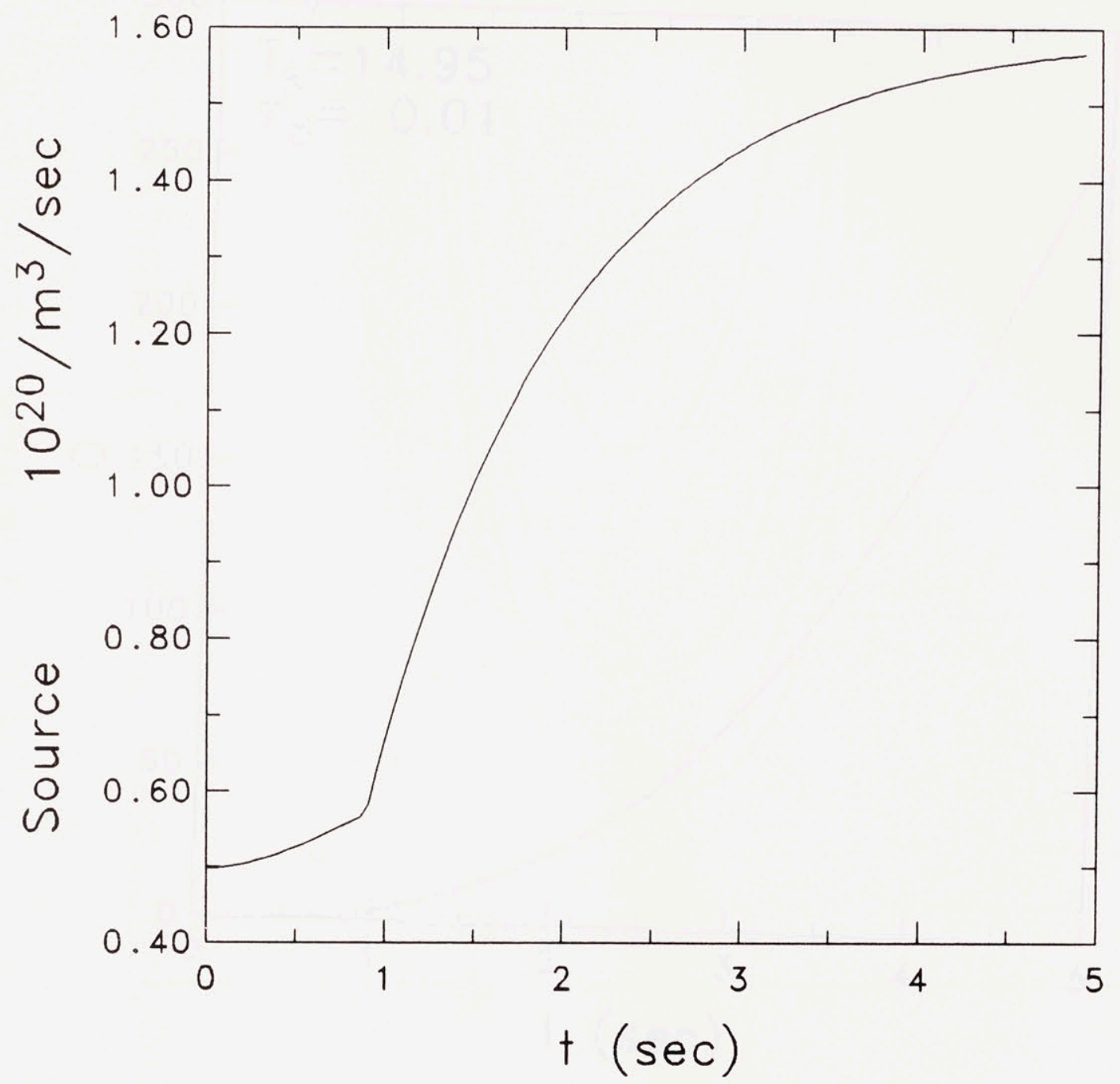

Figure 4.17: The particle source rate required to maintain the density evolution shown in Fig. 4.15 


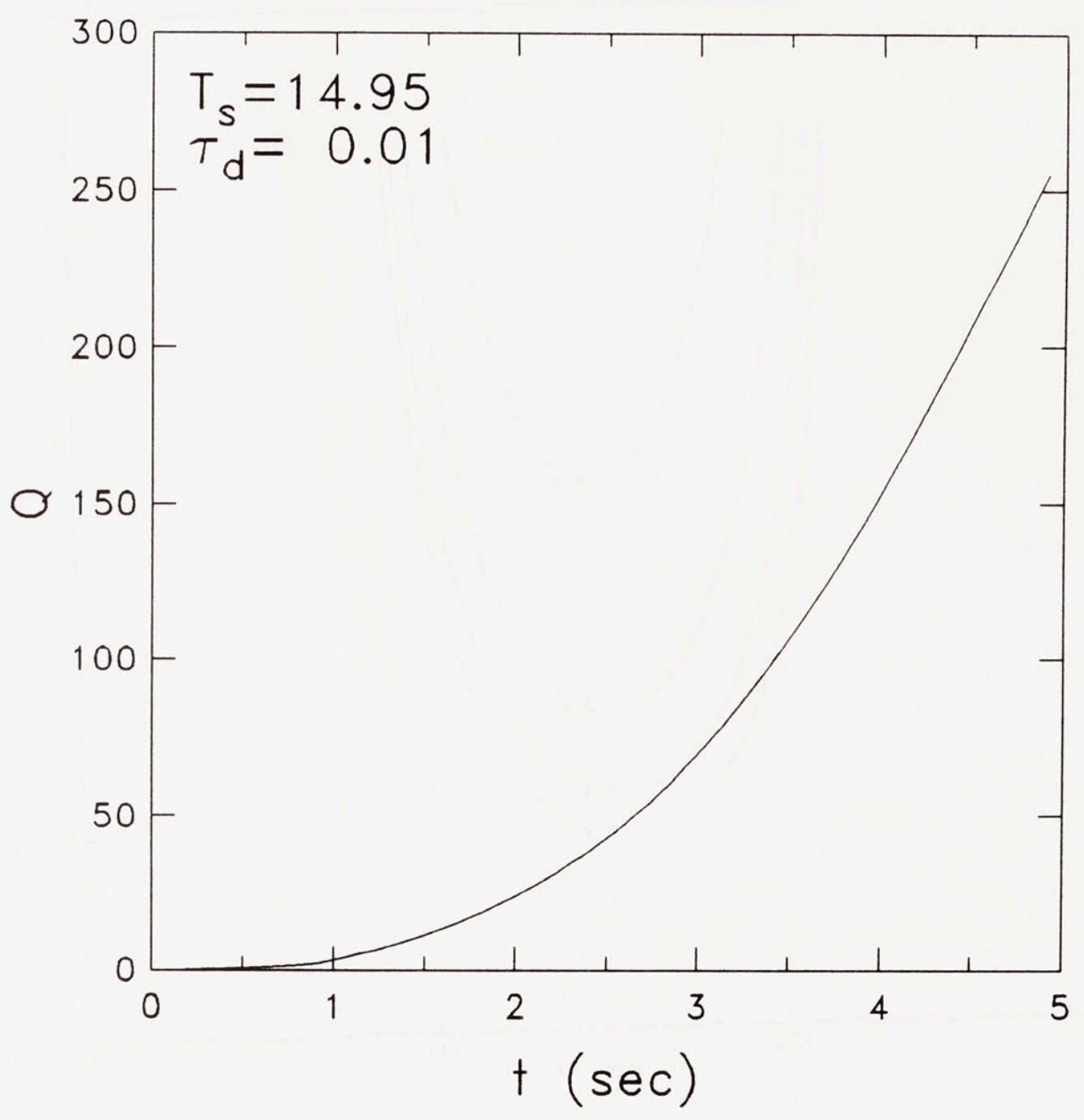

Figure 4.18: Time evolution of the thermonuclear $\mathrm{Q}$ corresponding to Fig. 4.14 


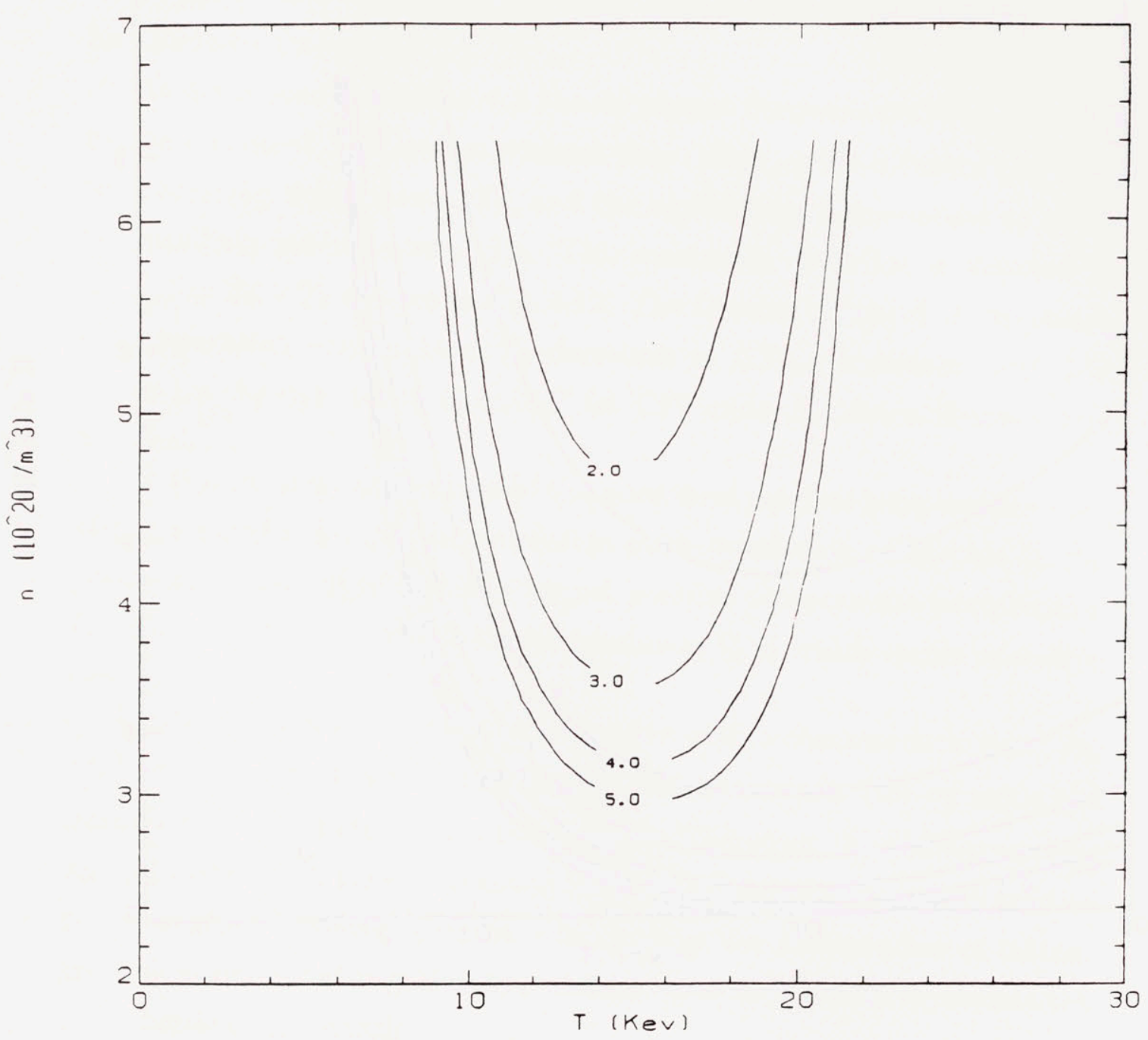

Figure 4.19: The e-folding time for the CIT unstable equilibria corresponding to Goldston H-mode confinement 


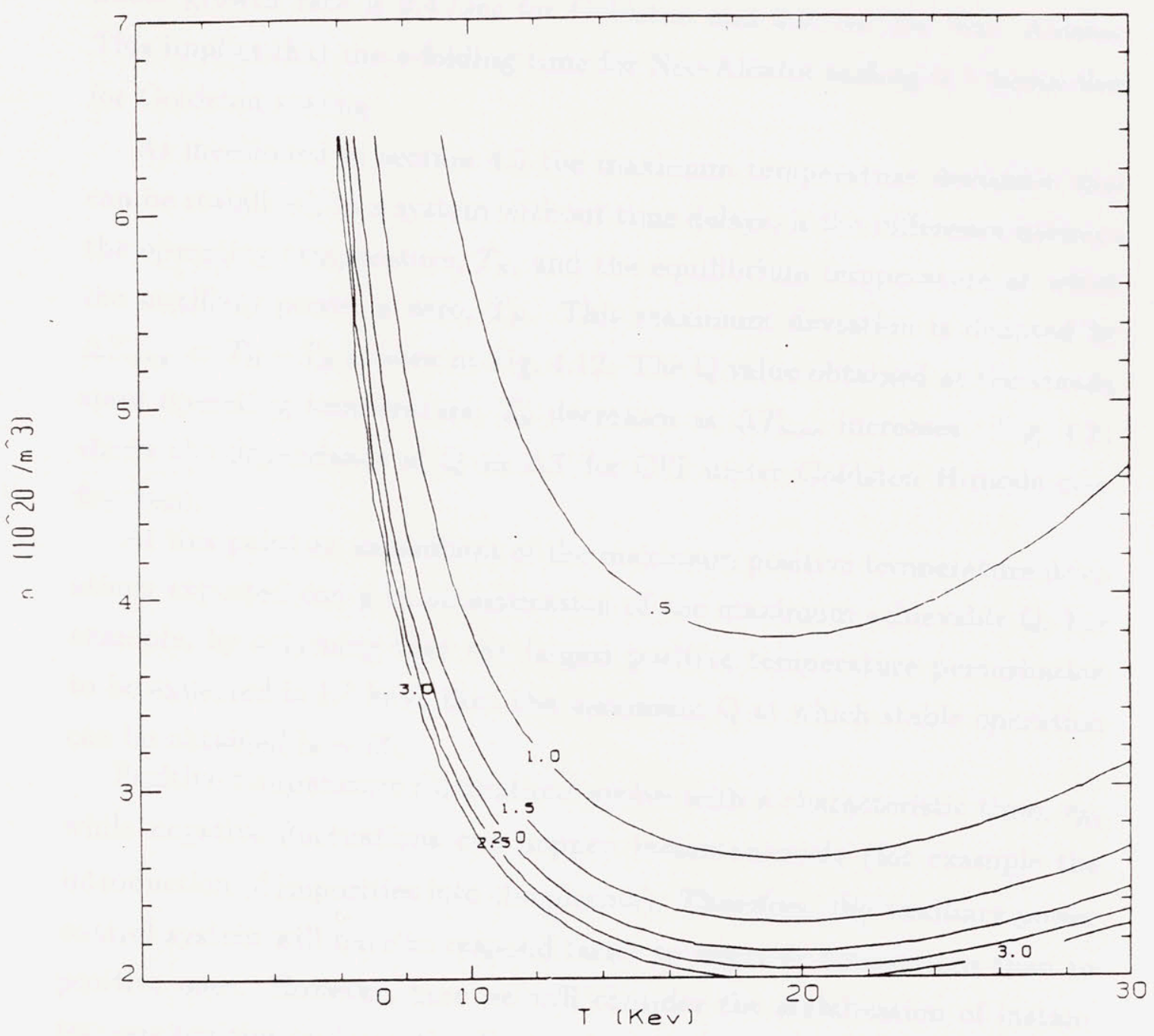

Figure 4.20: The e-folding time for the CIT unstable equilibria corresponding to Neo-Alcator confinement 
linear growth rate is $0.4 / \mathrm{sec}$ for Goldston and $2.0 / \mathrm{sec}$ for Neo- Alcator. This implies that the e-folding time for Neo-Alcator scaling is 5 times that for Goldston scaling.

As mentioned in section 4.5 the maximum temperature deviation that can be stabilized, in a system without time delays, is the difference between the operating temperature, $T_{S}$, and the equilibrium temperature at which the auxiliary power is zero, $T_{B}$. This maximum deviation is denoted by $\Delta T_{\max }=T_{B}-T_{S}$ as seen in Fig. 4.12. The $\mathrm{Q}$ value obtained at the steady state operating temperature, $T_{S}$ decreases as $\Delta T_{\max }$ increases. Fig. 4.21 shows the dependance of $\mathrm{Q}$ on $\Delta T$ for CIT under Goldston H-mode confinement.

At this point an assessment of the maximum positive temperature deviations expected can give an estimation of the maximum achievable Q. For example, by assuming that the largest positive temperature perturbation to be expected is $1.5 \mathrm{keV}$, then the maximum $\mathrm{Q}$ at which stable operation can be obtained is $\sim 65$.

Positive temperature fluctuations evolve with a characteristic time, $\tau_{R}$, while negative fluctuations can happen instantaneously (for example the introduction of impurities into the plasma). Therefore, the auxiliary power control system will have to respond faster to negative fluctuations than to positive ones. However, here we will consider the stabilization of instantaneous positive and negative fluctuations since such a case represents the most demanding scenario.

The feedback system, including the diagnostic system and the auxiliary power delivery system is characterized by the delay time $\tau_{d}$. For a certain feedback law, the maximum positive fluctuations to be stabilized as well as the maximum steady state $Q$ depends on the magnitude of $\tau_{d}$. By assuming a feedback law of the form given by Eq. 4.7 with $T_{1}=11.0, T_{2}=14.0$ and $\lambda=2.0$ the stabilization of an instantaneous $1.5 \mathrm{keV}$ positive temperature fluctuation is shown in Fig. 4.22, under three different values of the feedback delay time $\tau_{d}$. For $\tau_{d}=0.4$ the system resembles an underdamped oscillator. 


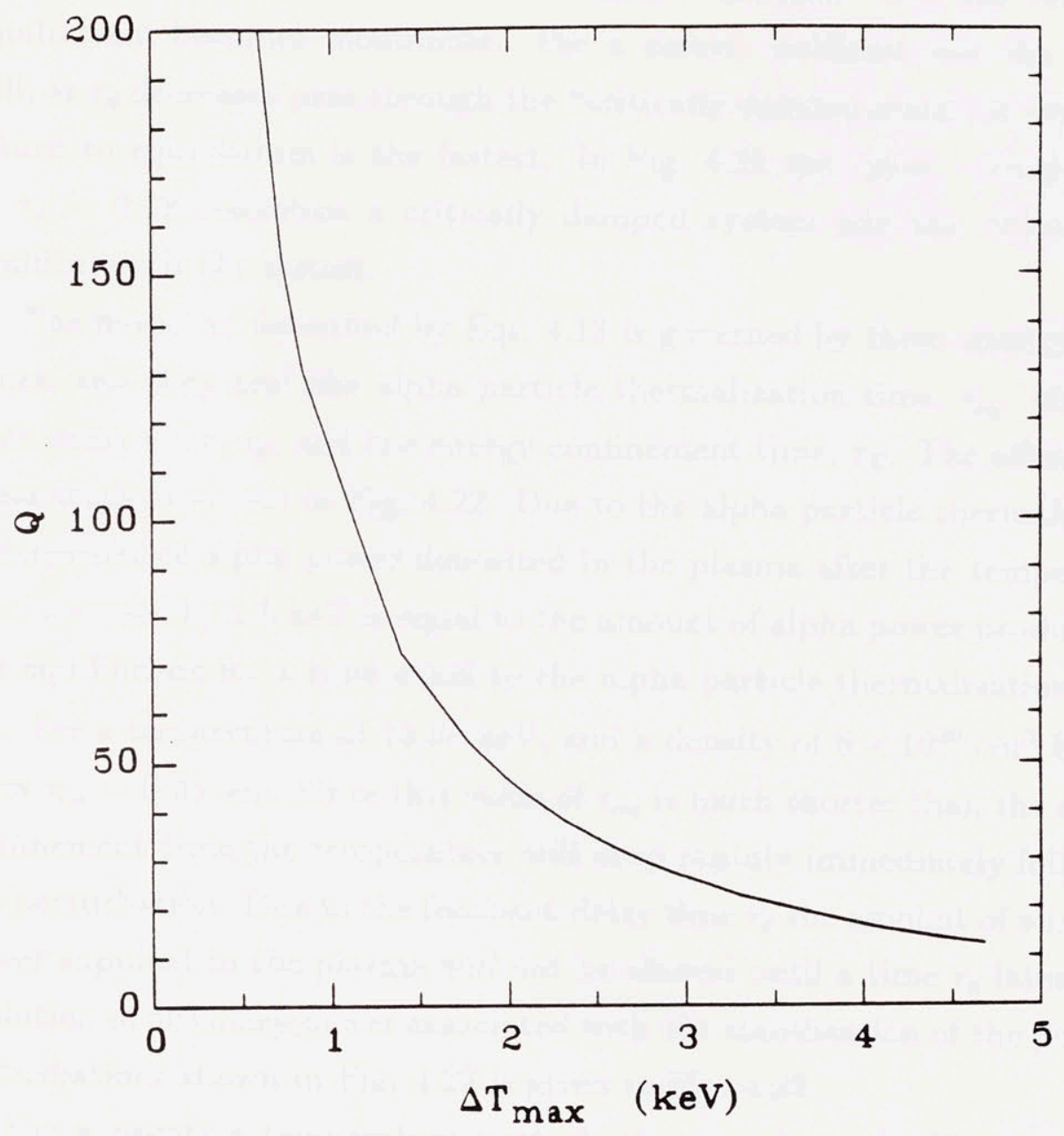

Figure 4.21: The dependance of the steady state $Q$ on the maximum temperature deviations stabilized in an ideal system 
As $\tau_{d}$ decreases the system becomes more "damped" and the return to equilibrium becomes monotonic. For a certain feedback law the system will, as $\tau_{d}$ decreases pass through the "critically damped state" at which the return to equilibrium is the fastest. In Fig. 4.22 the curve corresponding to $\tau_{d}=0.12$ resembles a critically damped system and the approach to equilibrium is the fastest.

The model as described by Eqs. 4.13 is governed by three characteristic times, and they are: the alpha particle thermalization time, $\tau_{\alpha e}$, the feedback delay time, $\tau_{d}$, and the energy confinement time, $\tau_{E}$. The effect of all these times is shown in Fig. 4.22. Due to the alpha particle thermalization the amount of alpha power deposited in the plasma after the temperature has increased by $1.5 \mathrm{keV}$ is equal to the amount of alpha power produced at the equilibrium for a time equal to the alpha particle thermalization time, $\tau_{\alpha e}$. For a temperature of $13.57 \mathrm{keV}$, and a density of $6 \times 10^{20} / \mathrm{m}^{3}$ Eq. 4.6 gives $\tau_{\alpha e}=0.05 \mathrm{sec}$. Since this value of $\tau_{\alpha e}$ is much shorter than the energy confinement time the temperature will drop rapidly immediately following the perturbation. Due to the feedback delay time $\tau_{d}$ the amount of auxiliary power supplied to the plasma will not be altered until a time $\tau_{d}$ later. The evolution of auxiliary power associated with the stabilization of the positive perturbations shown in Fig. 4.22 is given in Fig. 4.23.

For a negative temperature perturbation, as shown in Fig. 4.24, the amount of alpha power deposited in the plasma immediately after the temperature drop corresponds to the amount produced at the equilibrium and thus it tends to increase the plasma temperature. Therefore, the alpha particle thermalization time has a stabilizing effect on both positive and negative temperature fluctuations.

Furthermore, the effect of the auxiliary power will not be seen until a time $\tau_{d}$ later and thus the temperature will decrease further after the alpha particle thermalization effect is observed, as it is clearly seen for the case of $\tau_{d}=0.4 \mathrm{sec}$. The evolution of auxiliary power associated with the stabilization of the negative perturbations shown in Fig. 4.24 is given in Fig. 4.25. 


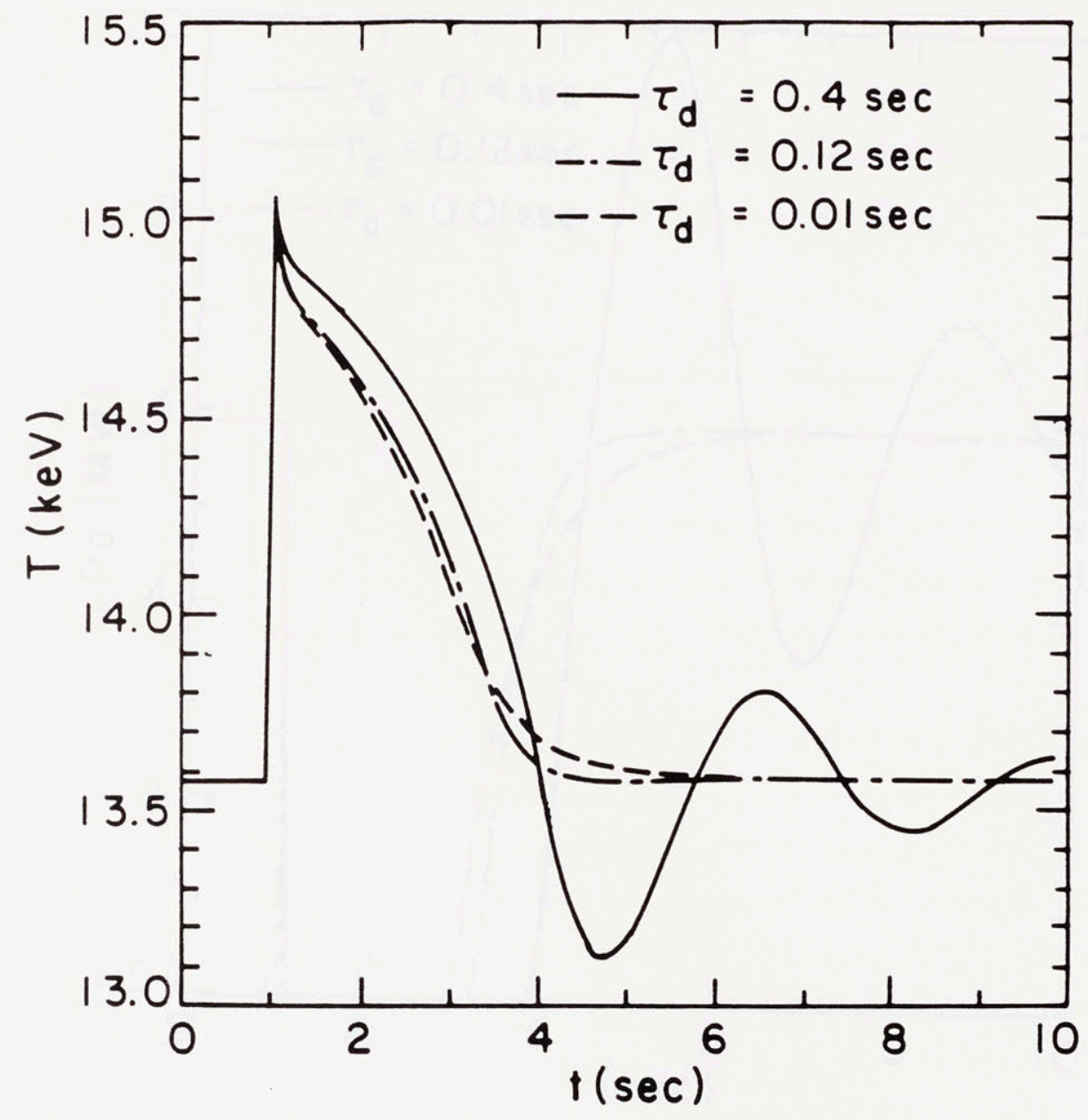

Figure 4.22: Stabilization of an instantaneous $1.5 \mathrm{keV}$ positive temperature deviation for different values of $\tau_{d}$ 


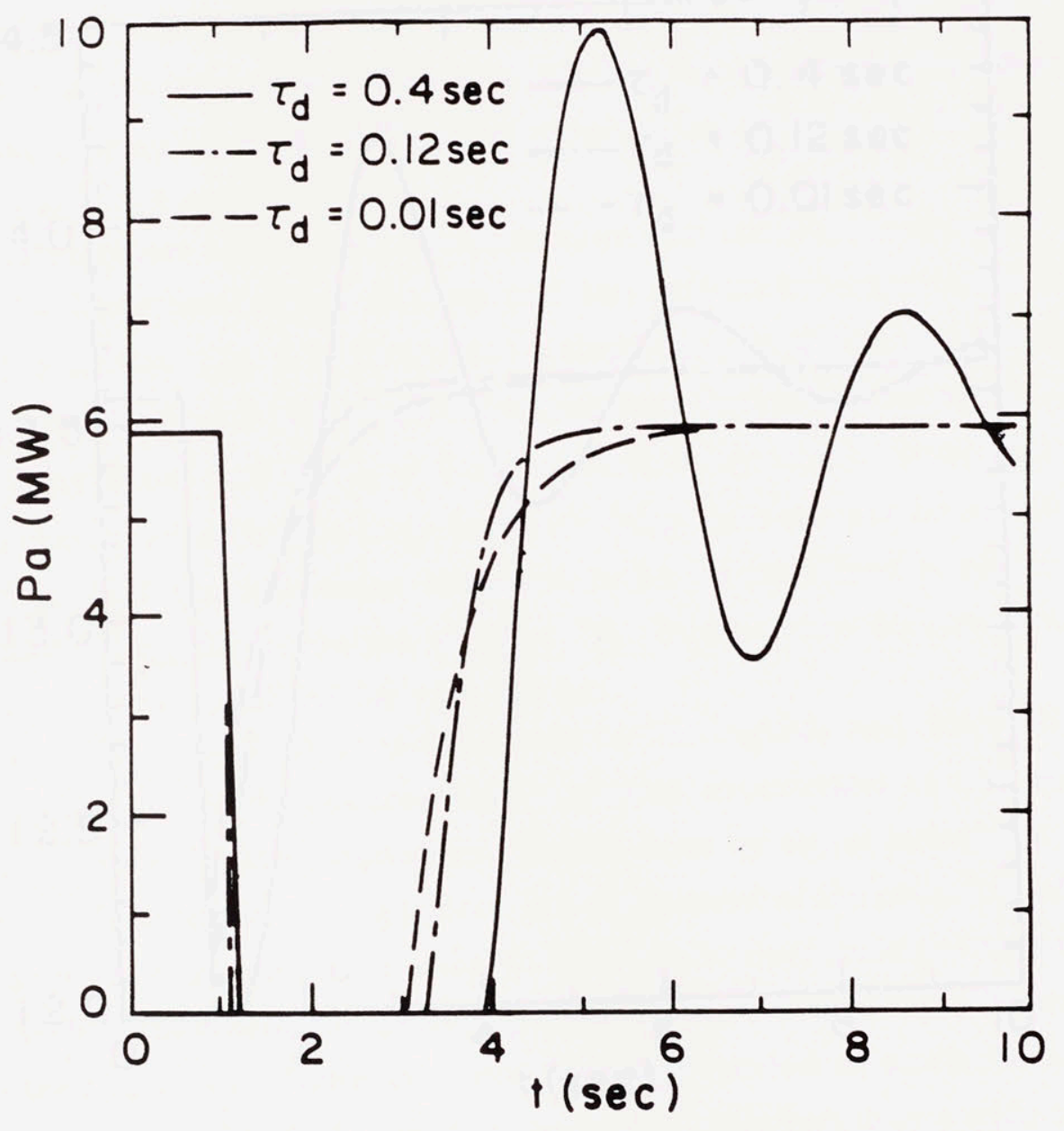

Figure 4.23: Evolution of auxiliary power required to stabilize a $1.5 \mathrm{keV}$ positive temperature deviation for various values of $\tau_{d}$. 


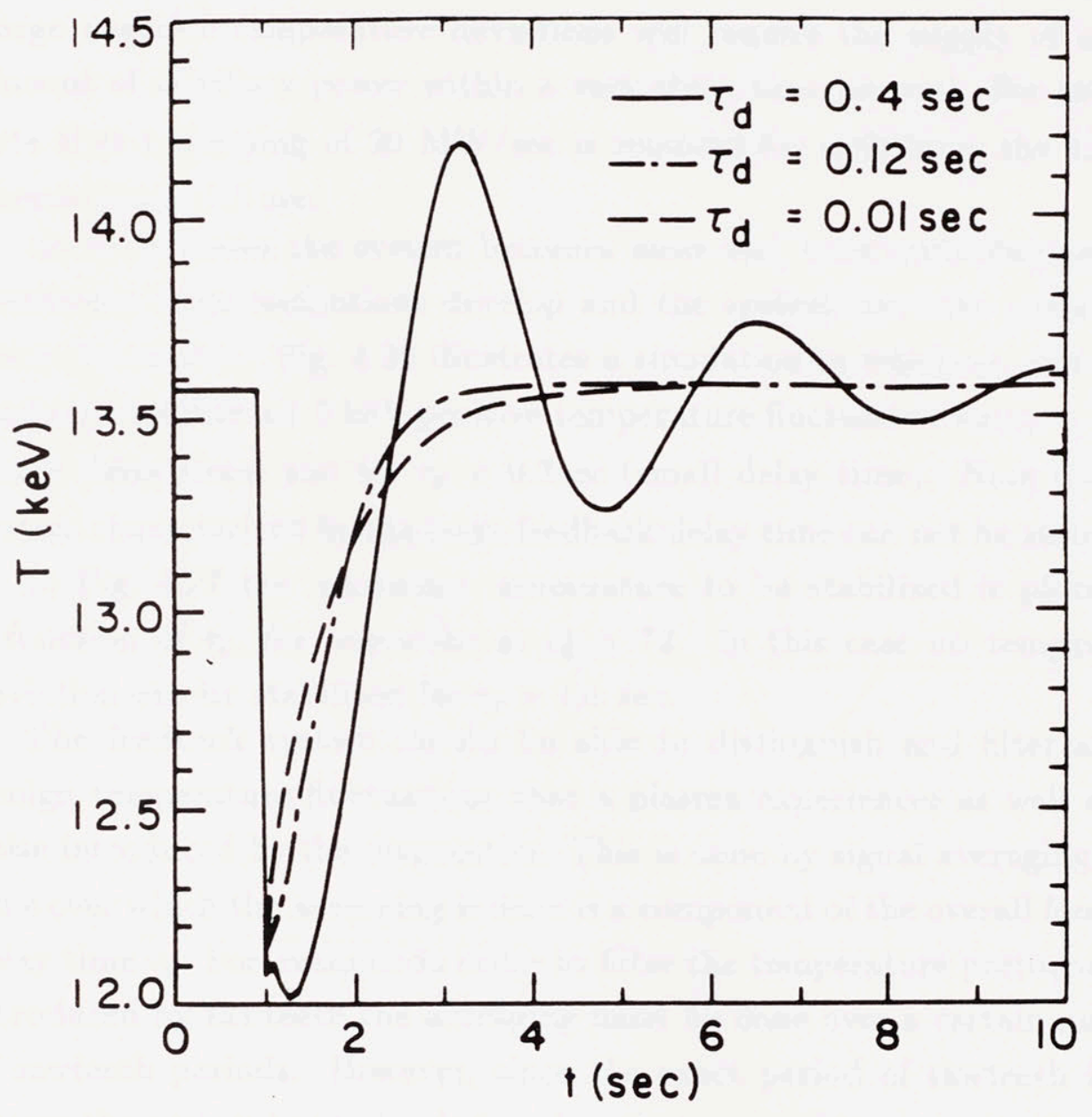

Figure 4.24: Stabilization of an instantaneous $1.5 \mathrm{keV}$ negative temperature deviation for different values of $\tau_{d}$ 
The auxiliary power ramping required for stabilizing negative perturbations should be compatible with the specifications of the auxiliary power system. Large negative temperature deviations will require the supply of a large amount of auxiliary power within a very short time interval. For example note that a ramping of $20 \mathrm{MW} / \mathrm{sec}$ is required for stabilizing the $1.5 \mathrm{keV}$ fluctuation in $1.5 \mathrm{sec}$.

As $\tau_{d}$ increases the system becomes more and more underdamped and eventually large oscillations develop and the system becomes unstable to every fluctuation. Fig. 4.26 illustrates a simulation in which an attempt is made to stabilize a $1.5 \mathrm{keV}$ positive temperature fluctuation for $\tau_{d}=1.5 \mathrm{sec}$ (large delay time) and for $\tau_{d}=0.2 \mathrm{sec}$ (small delay time). Note that the system characterized by the large feedback delay time can not be stabilized.

In Fig. 4.27 the maximum temperature to be stabilized is plotted as a function of $\tau_{d}$, for operation at $\mathrm{Q}=72$. In this case no temperature deviation can be stabilized for $\tau_{d}>1.5 \mathrm{sec}$.

The feedback system should be able to distinguish and filter all the benign temperature fluctuations that a plasma experiences as well as the noise introduced by the diagnostics. This is done by signal averaging. The time over which the averaging is done is a component of the overall feedback delay time $\tau_{d}$. For example in order to filter the temperature perturbations introduced by sawteeth the averaging must be done over a certain number of sawteeth periods. However, since the exact period of sawteeth is not known the system has to be designed so that it stabilizes over a wide range of periods without violating the maximum limit on $\tau_{d}$. A complete study of this phenomenon requires the development of a sawtooth model in the context of one dimensional transport. Such a model is developed in chapter 6.

\subsection{Conclusions}

Auxiliary power modulation can be used for operating CIT. By supplying auxiliary power to the plasma and appropriately modulating it he tokamak operating space can be mapped, the desired operating point can be 


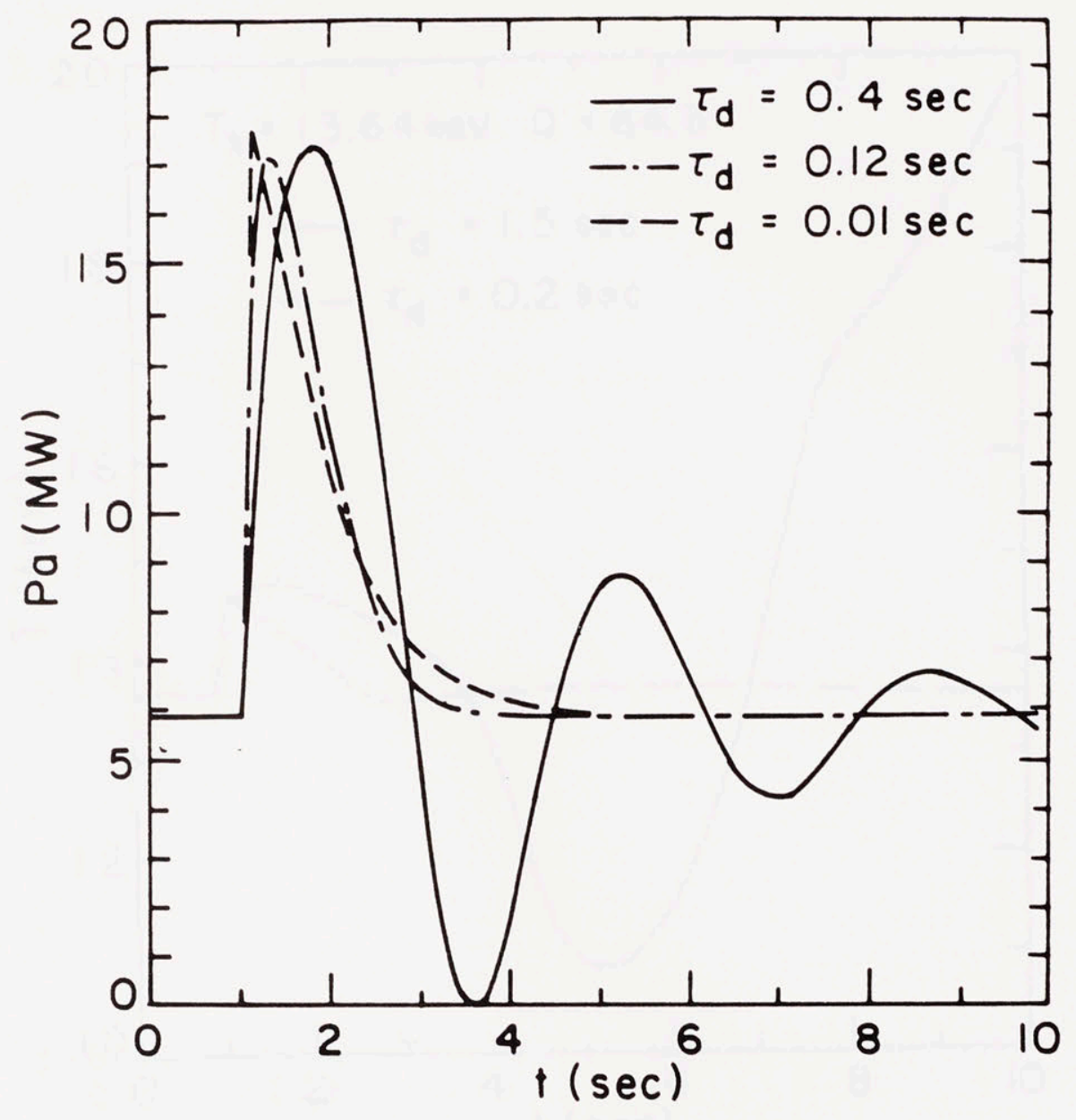

Figure 4.25: Evolution of auxiliary power required to stabilize a $1.5 \mathrm{keV}$ negative temperature deviation for various values of $\tau_{d}$. 


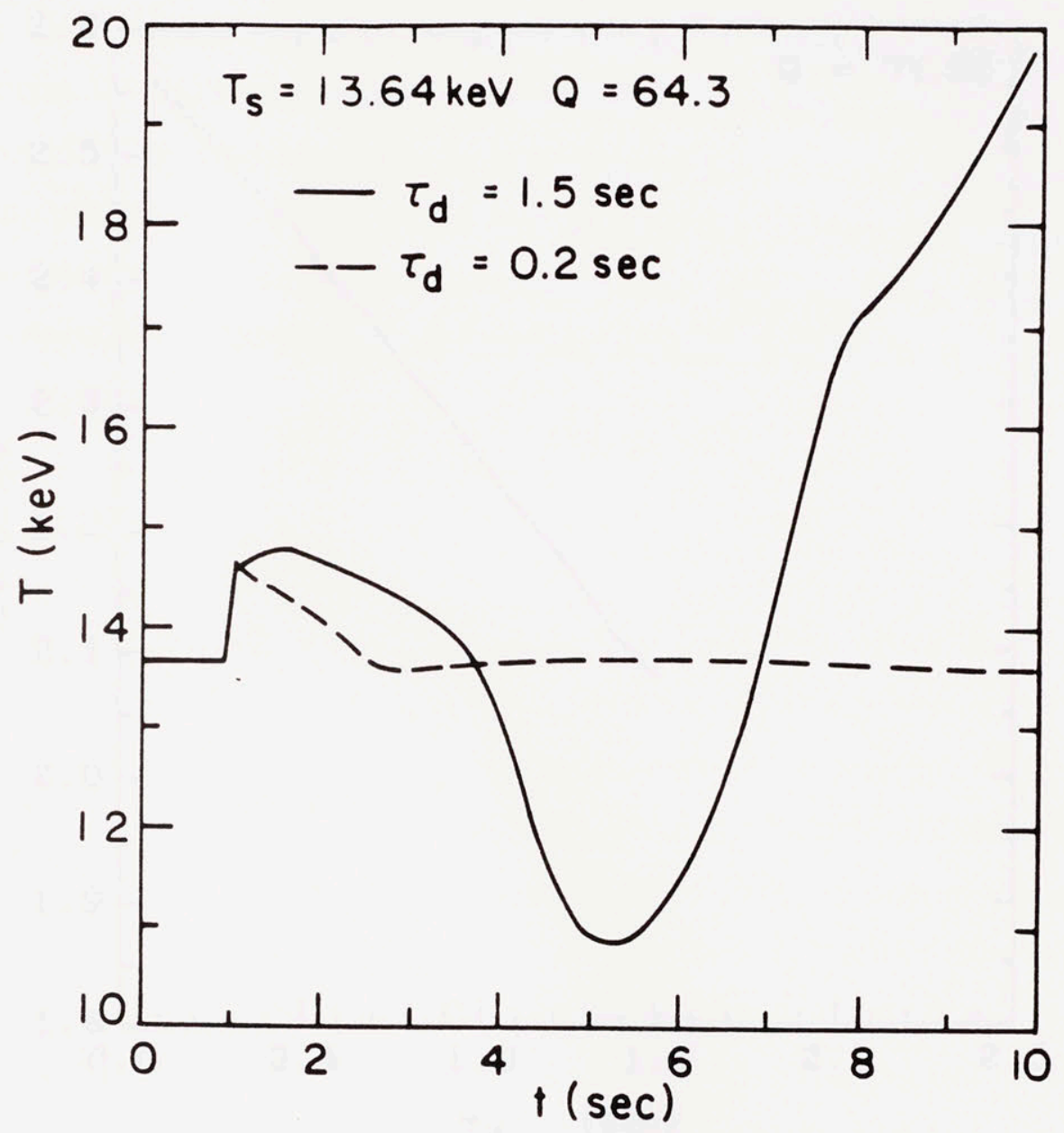

Figure 4.26: Simulation of a control system characterized a large delay time (solid line) and a small delay time (dashed line) 


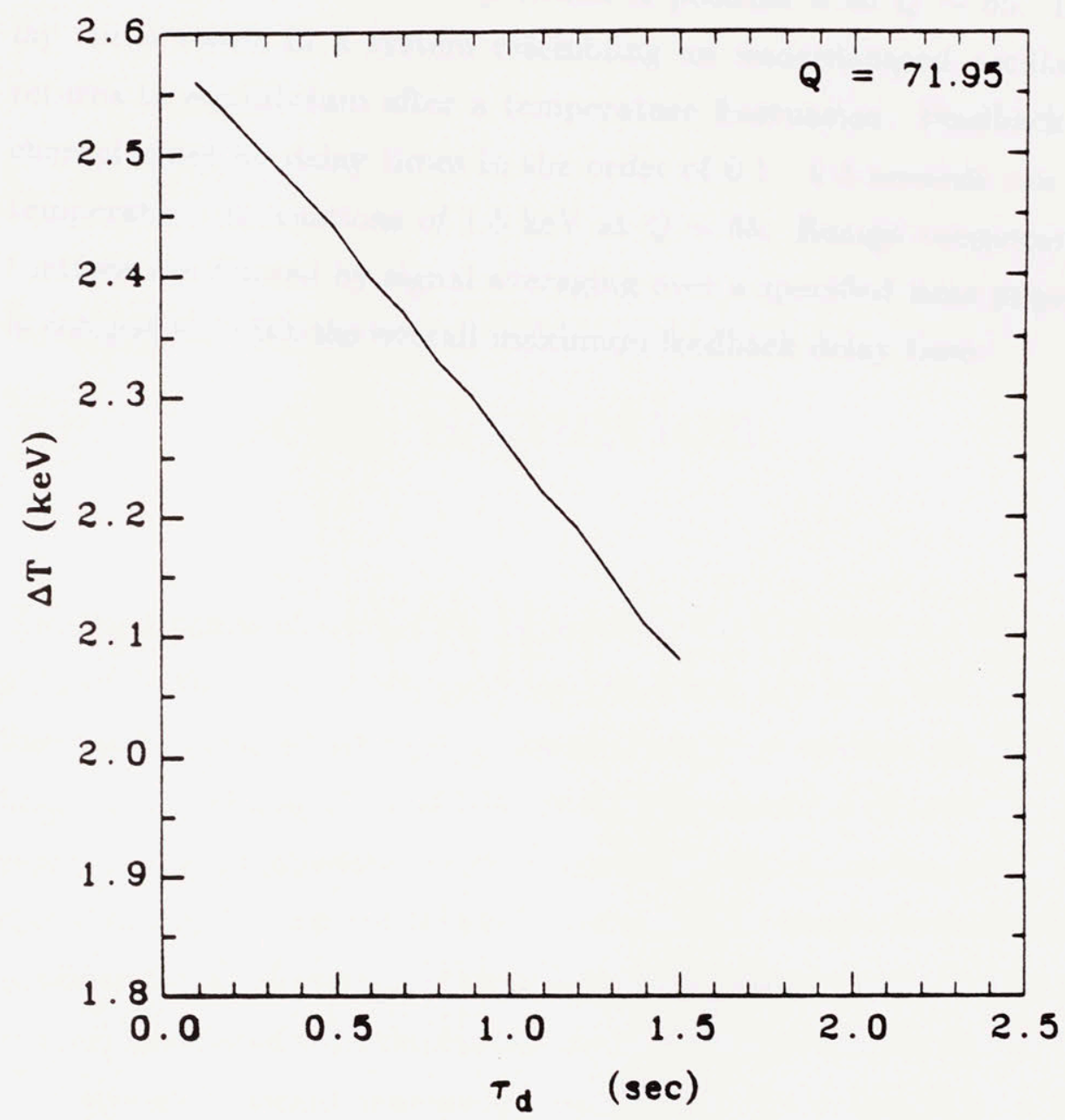

Figure 4.27: The maximum temperature deviation to be stabilized as a function of the feedback delay time $\tau_{d}$ 
reached, and control about the operating point can be achieved. It has been estimated that stable operation is possible with $Q \sim 65$. Large delay times result in a system resembling an underdamped oscillator as it returns to equilibrium after a temperature fluctuation. Feedback systems characterized by delay times in the order of $0.1-0.3$ seconds can stabilize temperature fluctuations of $1.5 \mathrm{keV}$ at $Q \sim 65$. Benign temperature fluctuations are filtered by signal averaging over a specified time period which is compatible with the overall maximum feedback delay time. 


\section{Chapter 5}

\section{Variational Formulation of the Transport Equation}

The importance of variational methods in the development of theoretical physics, both classical and quantum (modern), has long been appreciated. Extremal principles have given rise to the energy concept which forms the basis for visualizing the behavior of complex mechanical systems. [47] For example the equations of motion, and thus the behavior, of the system shown in Fig. 5.1 can not be easily deduced from simple force displacement considerations. However, by considering the energy (both kinetic and potential) associated with the system the equations of motion can be deduced in a straight forward manner by considering the extremal of a function "the Lagrangian" which is related to macroscopic energy content of the system.[48]

In addition to this conceptual advantage, extremal "variational" principles can be effective in the construction of solutions to mechanical problems. In this case, the equations describing the behavior of the system are known apriori and their solution, under certain constraints (boundary conditions, and initial conditions), is obtained through the use of variational principles.[49] In this thesis the variational formulation is used to obtain solutions to the equations describing the transport of mass, energy, and flux in 


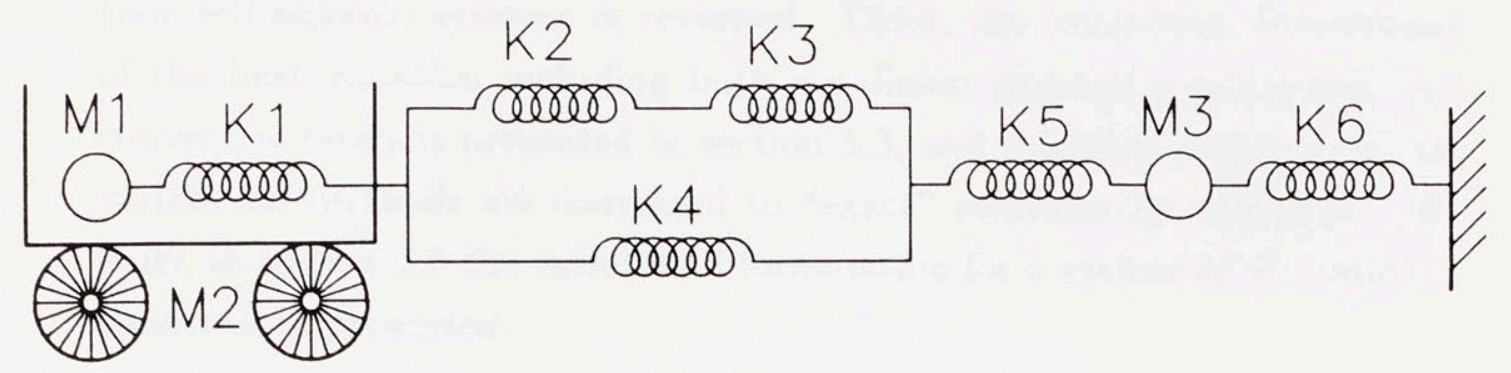

Figure 5.1: A typical mechanical system whose equations of motion are difficult to obtain by simply considering force balance relations. The equations can be obtained easily by considering the Lagrangian of the system

a tokamak plasma. The primary motivation for using the variational formulation to obtain solutions to the plasma transport equations is the decrease in computer time over standard finite differencing techniques. In general, a savings in execution time between a factor of $10-50$ can be realized. As a result one dimensional transport models may be incorporated in systems codes enabling the investigation of profile effects in the various parametric studies. Also such a fast transport model can be used as a between shot diagnostic in existing and future tokamak experiments.

Since transport equations in general characterize dissipative systems, the Lagrangian formulation of such a problem falls outside the "classical" range of variational applications.[48,50] In general the Lagrangian formulation of a dissipative system may be considered an extension of classical variational calculus to non-self-adjoint problems. This extension is made possible by applying the variational principle to the fluctuation alone.

In this chapter the variational formulation is presented in its generality. First, a brief review of the variational procedure, by which solutions to a certain set of equations can be obtained, is presented in section 5.1. Second, 
in section 5.2 the theory behind the variational formulation for dissipative (non-self-adjoint) systems is reviewed. Third, the variational formulation of the heat equation including both non-linear thermal conductivity and convective terms is presented in section 5.3, and solutions obtained by the variational methods are compared to "exact" solutions in section 5.5. Finally, in section 5.6 the variational formulation for a system of $N$ transport equations is presented.

\subsection{Variational formulation: General Re- marks}

The equations describing the behavior of a physical system are put in the variational form by introducing the integral

$$
\mathcal{L}=\int L d x
$$

where $L$, the Lagrangian density, is a function of functions [51] of the basic system parameters (i.e. space and time), and the generalized coordinates (i.e. dependent variables) $\alpha_{1}, \cdots, \alpha_{n}$.

$$
L=L\left(\alpha_{j} ; f(x, t)\right) \quad(j=1, \cdots, n)
$$

where $f(x, t)$ is the function characterising the system.

In general, the solution of physical problems via the variational formulation is a three step process. First, the functional $\mathcal{L}$ "Lagrangian" must be found whose first variation, $\delta \boldsymbol{L}$, yields the equation(s) describing the system as its "Euler equation". Second, a set of functions characterizing the solution must be determined. These "trial functions", which are given in terms of certain number of undetermined coefficients, the generalized coordinates, $\alpha_{1}, \cdots, \alpha_{n}$, are in turn substituted into the functional $\mathcal{L}$. Third, by taking the variations

$$
\frac{\delta \mathcal{L}}{\delta \alpha_{j}}=0 \quad(j=1, \cdots, n)
$$


a set of equations (differential or algebraic) are derived whose solution gives the values of $\alpha_{j}$ required for making the Lagrangian stationary.[47,49] Once the third step has been completed the solution is found by substituting the values of $\alpha_{j}$ into the assumed trial functions.

In section 5.3, the variational formulation of the heat equation will be obtained by following the above three steps.

\subsection{Lagrangian Formulation of Dissipative Systems}

It is known from studies of classical thermodynamics that entropy production gives a measure of the approach to equilibrium of physical systems excibiting reversible processes.[52] It is then important to investigate whether there is any function which for the case of dissipative systems could correspond to the role that the Lagrangian function plays in the development of classical and quantum mechanics.

It has been shown that for dissipative systems there exists the quantity

$$
d \Phi=\int_{V} \sum_{j} F_{j} d Q_{j} d V \leq 0
$$

which holds during the time evolution of the system without reference to the relation between the forces $F_{j}$ and the fluxes $Q_{j}$. [53,54] The inequality given by Eq. (5.4) is so general that it has been called a universal evolution criterion valid throughout the whole range of macroscopic physics.[55] In general the function $\Phi$ is called the "local potential". This local potential if it exists for the physical system under investigation is equivalent to the Lagrangian used in classical mechanics. By investigating the simple heat equation it is shown that there is a function corresponding to the heat equation which corresponds to Eq. (5.4).

The simple heat conduction equation of fixed density normalized to one is given by 


$$
c_{v} \frac{\partial T}{\partial t}=-\frac{\partial q_{j}}{\partial x_{j}}
$$

with $q_{j}$ representing the heat flux, and where $c_{v}$ is the specific heat.

By considering the domain of solutions of Eq. (5.5) within a volume $V$ with a boundary surface $S$, the families of temperature distributions of the form

$$
T=\widehat{T}\left(x_{j}, t\right)+\delta T\left(x_{j}, t\right)
$$

where $\widehat{T}$ is the appropriate macroscopic temperature distribution and where $\delta T$ are small variations around the macroscopic distribution represent the solution of Eq. (5.5). For the Fourier law

$$
q_{j}=-\kappa(T) \frac{\partial T}{\partial x_{j}}
$$

the thermal conductivity $\kappa$ may be written as

$$
\kappa(T)=\kappa(\widehat{T}+\delta T)=\widehat{\kappa}+\delta \kappa
$$

and similarly the specific heat may be written as

$$
c_{v}=\widehat{c}_{v}+\delta c_{v}
$$

By multiplying Eq. (5.5) by $-\delta T$ and by substituting Eq. (5.7) it follows that

$$
-c_{v}\left(\frac{\partial \widehat{T}}{\partial t}+\frac{\partial(\delta T)}{\partial t}\right) \delta T=-\frac{\partial}{\partial x_{j}}\left(\kappa \frac{\partial T}{\partial x_{j}}\right) \delta T
$$

which reduces to

$$
-c_{v} \frac{\partial \delta T}{\partial t} \delta T=c_{v} \frac{\partial \widehat{T}}{\partial t} \delta T-\frac{\partial}{\partial x_{j}}\left(\kappa \frac{\partial T}{\partial x_{j}} \delta T\right)+\kappa \frac{\partial T}{\partial x_{j}} \frac{\partial \delta T}{\partial x_{j}}
$$


and which is equivalent to

$$
-\frac{1}{2} \frac{\partial(\delta T)^{2}}{\partial t}=\frac{\partial \widehat{T}}{\partial t} \delta T-\frac{1}{c_{v}} \frac{\partial}{\partial x_{j}}\left(\kappa \frac{\partial T}{\partial x_{j}} \delta T\right)+\frac{\kappa}{c_{v}} \frac{\partial T}{\partial x_{j}} \frac{\partial \delta T}{\partial x_{j}}
$$

Integrating Eq. (5.12) over the volume $V$ results in

$$
\begin{gathered}
-\frac{1}{2} \frac{\partial}{\partial t} \int_{V}(\delta T)^{2} d V=\int_{V}\left[\frac{\partial \widehat{T}}{\partial t} \delta T+\right. \\
\left.\frac{\kappa}{2 c_{v}} \delta\left(\frac{\partial T}{\partial x_{j}}\right)^{2}+\kappa \frac{\partial T}{\partial x_{j}} \delta T \frac{\partial}{\partial x_{j}}\left(\frac{1}{c_{v}}\right)\right] d V \\
-\int_{S} \frac{\kappa}{c_{v}} \frac{\partial T}{\partial x_{j}} \delta T d S
\end{gathered}
$$

and by Integrating Eq. (5.13) over time the following results

$$
\begin{aligned}
-\frac{1}{2} \int_{V}(\delta T)^{2} d V=\int_{t} \int_{V}\left[\frac{\partial \widehat{T}}{\partial t} \delta T\right. & \left.+\frac{\kappa}{2 c_{v}} \delta\left(\frac{\partial T}{\partial x_{j}}\right)^{2}+\kappa \frac{\partial T}{\partial x_{j}} \delta T \frac{\partial}{\partial x_{j}}\left(\frac{1}{c_{v}}\right)\right] d V d t \\
& -\int_{t} \int_{S} \frac{\kappa}{c_{v}} \frac{\partial T}{\partial x_{j}} \delta T d S d t
\end{aligned}
$$

Since the left hand side term of the above equation is negative definite, the right hand side is always less than or equal to zero. Assuming that $\delta T \ll \widehat{T}$ and subsituting Eq. (5.8) into the right hand side of Eq. (5.14) gives to the first order

$$
\int_{t} \int_{V}\left[\frac{\partial \widehat{T}}{\partial t} \delta T+\widehat{\kappa} \frac{\partial}{\partial x_{j}}\left(\frac{1}{\widehat{c}_{v}} \delta T\right) \frac{\partial T}{\partial x_{j}}\right] d V d t-\int_{t} \int_{S} \frac{\widehat{\kappa}}{\widehat{c}_{v}} \frac{\partial T}{\partial x_{j}} \delta T d S d t \leq 0
$$

If the temperature is specified on the boundary $S$ (i.e. if $\delta T=0$ on the boundary) or if the fluxes across the boundary are zero the surface integral in Eq. (5.15) vanishes. Therefore the variation 


$$
\int_{t} \int_{V}\left[\frac{\partial \widehat{T}}{\partial t} \delta T+\widehat{\kappa} \frac{\partial}{\partial x_{j}}\left(\frac{\delta T}{c_{v}}\right) \frac{\partial T}{\partial x_{j}}\right] d V d t \leq 0
$$

is always less than zero, and it is equal to zero when the temperature distribution corresponding to $\widehat{T}$ is reached.

Similar arguments can be developed even if the time integration of Eq. (5.13) is not performed. In that case the quantity

$$
\int_{V}\left[\frac{\partial \widehat{T}}{\partial t} \delta T+\widehat{\kappa} \frac{\partial}{\partial x_{j}}\left(\frac{\delta T}{c_{v}}\right) \frac{\partial T}{\partial x_{j}}\right] d V
$$

can be positive or negative but it becomes zero when the temperature corresponding to the macroscopic distribution $\widehat{T}$ is reached. Therefore, the achievement of the macroscopic distribution $\widehat{T}$ can be characterized by the extremum condition

$$
\left.\frac{\delta \boldsymbol{L}(\widehat{T}, T)}{\delta T}\right|_{\widehat{T}}=0
$$

with the subsidiary condition

$$
T=\widehat{T}
$$

The functional $\mathcal{L}$ is given by

$$
\mathcal{L}=\int_{V}\left[T\left(\frac{\partial \widehat{T}}{\partial t}\right)+\frac{1}{2} \frac{\widehat{\kappa}}{\widehat{c}_{\boldsymbol{v}}}\left(\frac{\partial T}{\partial x_{j}}\right)\right] d V
$$

where for simplicity it has been assumed that the specific heat $c_{v}$ is independent of temperature, $T$.

The functional $\mathcal{L}$ will henceforth be called the Lagrangian of the system. The optimization operation indicated by Eq. (5.18) shows that the functional $\mathcal{L}$ has as its Euler-Langrange equation the original heat transport equation (5.5). A detailed proof of this point is given in the next section for a specific example. 


\subsection{Variational formulation of the heat equa- tion}

In order to gain insight into the workings of the variational formulation, and appreciation of its power in finding solutions to the diffusion problem, various simple examples are presented.

The heat transport equation, in cylindrical geometry of unit radius and of normalized constant density, is given by

$$
\begin{aligned}
\frac{\partial T(\rho, t)}{\partial t}= & \frac{1}{\rho} \frac{\partial}{\partial \rho} \rho\left[\kappa(T, \rho, t) \frac{\partial T(\rho, t)}{\partial \rho}-v(T, \rho, t) T(\rho, t)\right] \\
& \quad+S(T, \rho, t) \\
\frac{\partial T(0, t)}{\partial \rho}= & 0 \\
T(1, t)= & 0 \\
T(\rho, 0)= & f(\rho)
\end{aligned}
$$

Note that Eq. (5.21) is, in general, non-linear since the thermal conductivity, $\kappa$, the convective velocity, $v$, and the source term, $S$ have been written as functions of temperature, $T$, space, $\rho$, and time $t$.

For the above problem the Lagrangian $\mathcal{L}$ is given by

$$
\mathcal{L}=\int_{A} L d A
$$

where the integration is over the cross sectional area of the cylinder, and the Lagrangian density $L$ is defined by

$$
L=T \frac{\partial \widehat{T}}{\partial t}+\frac{1}{2} \widehat{\kappa}\left(\frac{\partial T}{\partial \rho}\right)^{2}-\widehat{v} \widehat{T} \frac{\partial T}{\partial \rho}-I
$$

where the quantity $I$ in the last term of Eq. (5.26) is given by

$$
I \equiv \int_{\widehat{T}}^{T} S d T
$$


where $\widehat{T}$ is the macroscopic temperature. Therefore, the Lagrangian $\mathcal{L}$ becomes

$$
\mathcal{L}=\int_{0}^{1} \int_{0}^{2 \pi} \rho d \rho d \theta\left[T \frac{\partial \widehat{T}}{\partial t}+\frac{1}{2} \widehat{\kappa}\left(\frac{\partial T}{\partial \rho}\right)^{2}-\widehat{v} \widehat{T} \frac{\partial T}{\partial \rho}-I\right]
$$

In the above equation it is assumed, following the formulation presented in section 5.2, that the temperature distribution $T$ is made up of the appropriate macroscopic temperature distribution $\widehat{T}$, plus small variations of the temperature, $\delta T$, about the distribution $\widehat{T}[50]$

$$
T=\widehat{T}(\rho, t)+\delta T(\rho, t)
$$

The thermal conductivity, $\kappa$, and the convective velocity, $v$, in Eq. (5.28), are arbitrary functions of temperature and are evaluated at the macroscopic temperature $\widehat{T}$ so they are appropriately labeled $\widehat{\kappa}$, and $\widehat{v}$.

The Lagrangian $\mathcal{L}$ is thus a function of both the total temperature $T$ and the macroscopic temperature $\widehat{T}$. The crucial feature to note is that in taking the variations of $\mathcal{L}$ the quantities accented with a $\left(^{(}\right)$are not subject to variation. The first variation of Eq. (5.28) gives

$$
\delta \mathcal{L}=2 \pi \int_{0}^{1}\left[\delta T \frac{\partial \widehat{T}}{\partial t}+\widehat{\kappa} \frac{\partial T}{\partial \rho} \frac{\partial(\delta T)}{\partial \rho}-\widehat{v} \widehat{T} \frac{\partial(\delta T)}{\partial \rho}-S \delta T\right] \rho d \rho
$$

where by assuming poloidal symmetry the integration over the poloidal angle has been performed. Since the relation between $\delta T$ and $\partial(\delta T) / \partial \rho$ is not known, a simple integration by parts of the second and third terms in the right hand side of Eq. (5.30) yields

$$
\begin{gathered}
\delta \mathcal{L}=\int_{0}^{1}\left[\frac{\partial \widehat{T}}{\partial t}-\frac{1}{\rho} \frac{\partial}{\partial \rho}\left(\rho \widehat{\kappa} \frac{\partial T}{\partial \rho}\right)+\frac{1}{\rho} \frac{\partial}{d \rho}(\rho \widehat{v} \widehat{T})-S\right] \delta T \rho d \rho(5.31) \\
+\left.\rho \widehat{\kappa} \frac{\partial T}{\partial \rho} \delta T\right|_{0} ^{1}-\left.\rho \widehat{v} \widehat{T} \delta T\right|_{0} ^{1}
\end{gathered}
$$


Where the factor of $2 \pi$, being a constant, has been neglected from the above equation.

From Eq. (5.23) it is seen that the value of the temperature, $T$, at the boundary of the cylinder $(\rho=1)$ has been specified (i.e. $\delta T(1, t)=0$ ). Therefore the last two terms in Eq. (5.31) are equal to zero. Now that the variation has been calculated, set $T=\widehat{T}, \widehat{v}=v, \kappa=\widehat{\kappa}$, and Eq. (5.31) becomes

$$
\delta \mathcal{L}=\int_{0}^{1}\left[\frac{\partial T}{\partial t}-\frac{1}{\rho} \frac{\partial}{\partial \rho}\left(\rho \kappa \frac{\partial T}{\partial \rho}\right)+\frac{1}{\rho} \frac{\partial}{\partial \rho}(\rho v T)-S\right] \delta T \rho d \rho
$$

Thus, in order for $\mathcal{L}$ to be stationary (i.e. $\delta \mathcal{L}=0$ ) for arbitrary variations, $\delta T$, it is necessary that the quantity within the square brackets in Eq. (5.32) must vanish. Therefore, the necessary and sufficient condition [47] for the vanishing of $\delta \mathcal{L}$ under arbitrary $\delta T$ variations is that $T$ satisfies the original equation (5.21). Therefore, the function $T$ which makes the Lagrangian stationary (i.e. $\delta \mathcal{L}=0$ ) is also a solution to the original equation. It has thus been shown that the "Euler equation" of the Lagrangian given by Eq. (5.28) is the equation characterizing the system under investigation.

The next step in the variational formulation requires the introduction of the trial function. Such a trial function must be capable of representing, or approximating, the solution of the differential equation. For the heat transport problem under investigation (Eqs. $(5.21,-5.24))$ a function which models a wide variety of profiles is

$$
T=T_{0}\left(1-\rho^{2}\right) \exp \left[\alpha_{1} \rho^{2}+\alpha_{2} \rho^{4}\right]
$$

The coefficients, $T_{0}(t), \alpha_{1}(t)$, and $\alpha_{2}(t)$, characterize the solution, and they are to be determined as a function of time. Fig. 5.2 demonstrates the flexibility provided by Eq. (5.33) in modeling profile shapes. Note that 


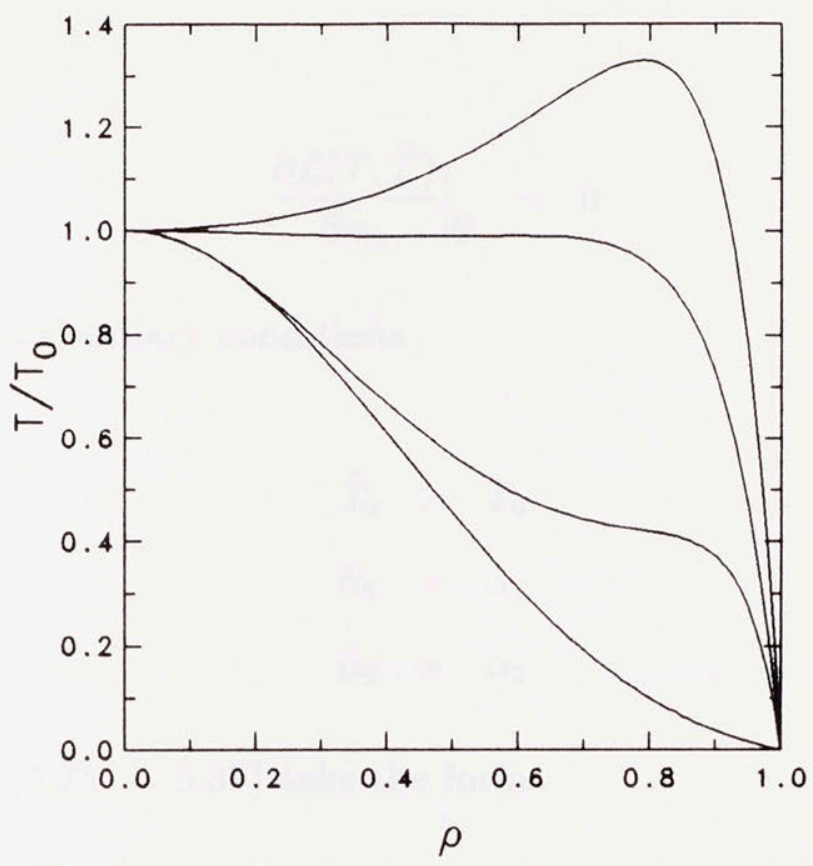

Figure 5.2: Profile shapes obtained for various values of the parameters $\alpha_{1}$, and $\alpha_{2}$. Note that profile shapes ranging from peaked to flat to hollow can be produced with the trial function Eq. (5.33).

steep, flat, and hollow profiles can be modeled by appropriately choosing the parameters $\alpha_{1}$, and $\alpha_{2}$.

In accordance with the variational principle the function $\widehat{T}$ in Eq. (5.28) is written as

$$
\widehat{T}=\widehat{T}_{0}\left(1-\rho^{2}\right) \exp \left[\widehat{\alpha}_{1} \rho^{2}+\widehat{\alpha}_{2} \rho^{4}\right]
$$

When computing the Euler-Langrange equations the parameters $T_{0}, \alpha_{1}$, and $\alpha_{2}$ are varied while $\widehat{T}_{0}, \widehat{\alpha}_{1}$, and $\widehat{\alpha}_{2}$ are held fixed. After the variation one sets $\widehat{T}_{0}=T_{0}, \widehat{\alpha}_{1}=\alpha_{1}$, and $\widehat{\alpha}_{2}=\alpha_{2}$. Specifically by substituting the trial function Eqs. $(5.33,5.34)$ into Eq. (5.28) the extremum of $\mathcal{L}$ in the $\left(T_{0}, \alpha_{1}, \alpha_{2}\right)$ space is found by taking the derivatives

$$
\begin{aligned}
& \left.\frac{\partial \mathcal{L}(T, \widehat{T})}{\partial T_{0}}\right|_{\widehat{T}}=0 \\
& \left.\frac{\partial \mathcal{L}(T, \widehat{T})}{\partial \alpha_{1}}\right|_{\widehat{T}}=0
\end{aligned}
$$




$$
\left.\frac{\partial \boldsymbol{L}(T, \widehat{T})}{\partial \alpha_{2}}\right|_{\widehat{T}}=0
$$

subject to the subsidiary conditions

$$
\begin{aligned}
& \widehat{T}_{0}=T_{0} \\
& \widehat{\alpha}_{1}=\alpha_{1} \\
& \widehat{\alpha}_{2}=\alpha_{2}
\end{aligned}
$$

Equations $(5.35-5.37)$ take the form

$$
\begin{gathered}
\int_{0}^{1}\left[\frac{\partial T}{\partial T_{0}} \frac{\partial T}{\partial t}+\kappa \frac{\partial T}{\partial \rho} \frac{\partial}{\partial \rho}\left(\frac{\partial T}{\partial T_{0}}\right)-v T \frac{\partial}{\partial \rho}\left(\frac{\partial T}{\partial T_{0}}\right)\right] \rho d \rho \\
-\int_{0}^{1}\left[S\left(\frac{\partial T}{\partial T_{0}}\right)\right] \rho d \rho=0 \\
\int_{0}^{1}\left[\frac{\partial T}{\partial \alpha_{1}} \frac{\partial T}{\partial t}+\kappa \frac{\partial T}{\partial \rho} \frac{\partial}{\partial \rho}\left(\frac{\partial T}{\partial \alpha_{1}}\right)-v T \frac{\partial}{\partial \rho}\left(\frac{\partial T}{\partial \alpha_{1}}\right)\right] \rho d \rho \\
-\int_{0}^{1}\left[S\left(\frac{\partial T}{\partial \alpha_{1}}\right)\right] \rho d \rho=0 \\
\int_{0}^{1}\left[\frac{\partial T}{\partial \alpha_{2}} \frac{\partial T}{\partial t}+\kappa \frac{\partial T}{\partial \rho} \frac{\partial}{\partial \rho}\left(\frac{\partial T}{\partial \alpha_{2}}\right)-v T \frac{\partial}{\partial \rho}\left(\frac{\partial T}{\partial \alpha_{2}}\right)\right] \rho d \rho \\
-\int_{0}^{1}\left[S\left(\frac{\partial T}{\partial \alpha_{2}}\right)\right] \rho d \rho=0
\end{gathered}
$$

Using the chain rule, the time derivative of $T$ becomes

$$
\frac{\partial T}{\partial t}=\frac{\partial T}{\partial T_{0}} \frac{d T_{0}}{d t}+\frac{\partial T}{\partial \alpha_{1}} \frac{d \alpha_{1}}{d t}+\frac{\partial T}{\partial \alpha_{2}} \frac{d \alpha_{2}}{d t}
$$

where for the trial functions under consideration 


$$
\begin{aligned}
& \frac{\partial T}{\partial T_{0}}=\frac{T}{T_{0}} \\
& \frac{\partial T}{\partial \alpha_{1}}=T \rho^{2} \\
& \frac{\partial T}{\partial \alpha_{2}}=T \rho^{4}
\end{aligned}
$$

Finally, the equations to be solved can be written in the matrix form

$$
\overrightarrow{\dot{U}}=\overleftrightarrow{\mathbf{A}}^{-1} \vec{R}
$$

Note that we have adapted the convention that the dot-operator $\left({ }^{\circ}\right)$ denotes differentiation with respect to time. In Eq. (5.48), $\overrightarrow{\dot{U}}$ is a column vector with components,

$$
\overrightarrow{\dot{U}}=\left[\begin{array}{c}
\dot{T}_{0} \\
\dot{\alpha}_{1} \\
\dot{\alpha}_{2}
\end{array}\right]
$$

$\overleftrightarrow{\mathrm{A}}$ is a $3 \times 3$ symmetric matrix

$$
\overleftrightarrow{\mathbf{A}}=\left[\begin{array}{lll}
A_{11} & A_{12} & A_{13} \\
A_{12} & A_{22} & A_{23} \\
A_{13} & A_{23} & A_{33}
\end{array}\right]
$$

whose elements are given by

$$
\begin{aligned}
& A_{11}=\frac{1}{T_{0}^{2}} \int_{0}^{1} T^{2} \rho d \rho \\
& A_{12}=\frac{1}{T_{0}} \int_{0}^{1} T^{2} \rho^{3} d \rho \\
& A_{13}=\frac{1}{T_{0}} \int_{0}^{1} T^{2} \rho^{5} d \rho
\end{aligned}
$$




$$
\begin{aligned}
& A_{22}=\int_{0}^{1} T^{2} \rho^{5} d \rho \\
& A_{23}=\int_{0}^{1} T^{2} \rho^{7} d \rho \\
& A_{33}=\int_{0}^{1} T^{2} \rho^{9} d \rho
\end{aligned}
$$

Note that the elements $A_{i j}$ are independent of the phenomenological coefficients $\kappa$, and $v$, as well as of the source term $S$. The dependance on $\kappa, v$, and $S$ is incorporated into the elements of the column vector $\vec{R}$

$$
\vec{R}=\left[\begin{array}{c}
R_{1} \\
R_{2} \\
R_{3}
\end{array}\right]
$$

where for a trial function of the form given by Eq. (5.33) the elements of $\vec{R}$ are

$$
\begin{aligned}
& R_{1}=\frac{1}{T_{0}} \int_{0}^{1}\left[-\kappa\left(\frac{\partial T}{\partial \rho}\right)^{2}+v T \frac{\partial T}{\partial \rho}+S T\right] \rho d \rho \\
& R_{2}=\int_{0}^{1}\left[-\kappa \frac{\partial T}{\partial \rho} \frac{\partial}{\partial \rho}\left(\rho^{2} T\right)+v T \frac{\partial}{\partial \rho}\left(\rho^{2} T\right)+S T \rho^{2}\right] \rho d \rho(5 \\
& R_{3}=\int_{0}^{1}\left[-\kappa \frac{\partial T}{\partial \rho} \frac{\partial}{\partial \rho}\left(\rho^{4} T\right)+v T \frac{\partial}{\partial \rho}\left(\rho^{4} T\right)+S T \rho^{4}\right] \rho d \rho(5
\end{aligned}
$$

Therefore, Eq. (5.48) represents a system of coupled non-linear ordinary differential equations in time. The variational formulation has thus converted the original partial differential equation in $(\rho, t)$ to a system of ordinary differential equations in $t$. Consequently, the system represented by Eq. (5.48) is less complex, and as we shall see this method is faster than the solution of difference equations required to approximate the partial differential equation.

In the next section we evaluate the validity of the variational formulation, in terms of accuracy, relative to solutions obtained by analytic means, and in terms of accuracy and speed, relative to solutions obtained by finite differencing. 


\subsection{Generalized Boundary Conditions}

If the solution of Eq. (5.21) is desired with non zero (constant or time dependent) edge boudary condition i.e. if Eq. (5.23) has the form

$$
T(1, t)=T_{1}(t)
$$

the Lagrangian given by Eq. (5.28) has to be modified in order to appropriately represent this situation. The alternate variational formulation which takes into account this boundary condition is

$$
\begin{array}{r}
\mathcal{L}=\int_{0}^{1} \rho d \rho\left[T \frac{\partial \widehat{T}}{\partial t}+\frac{1}{2} \widehat{\kappa}\left(\frac{\partial T}{\partial \rho}\right)^{2}-\widehat{v} \widehat{T} \frac{\partial T}{\partial \rho}-I\right] \\
-\left.\rho \widehat{\kappa} \frac{\partial \widehat{T}}{\partial \rho} T\right|_{\rho=1}+\left.\rho \widehat{v} \widehat{T} T\right|_{\rho=1}
\end{array}
$$

The Lagrangian given by Eq. (5.62) contains the "natural boundary condition" of the system, $[4]$ and it has the advantage of allowing trial functions to be substituted that do not satisfy the boundary condition Eq. (5.61).

The trial function, Eq. (5.33), can incorporate the non-zero boundary condition Eq. (5.61) with the following modification

$$
T=T_{0}\left(1-\gamma \rho^{2}\right) \exp \left[\alpha_{1} \rho^{2}+\alpha_{2} \rho^{4}\right]
$$

where $\gamma$ is given by

$$
\gamma=1-\frac{T_{1}(t)}{T_{0} \exp \left[\alpha_{1}+\alpha_{2}\right]}
$$

The remaining analysis for the formulation of the problem proceeds exacly as the case with zero boundary conditions presented in section 5.3. The only difference is the addition of the two new boundary terms and the incorporation of the parameter $\gamma$ in the formulation. 


\subsection{Test problems}

By assuming various forms for the thermal conductivity $\kappa$, the convective velocity $v$, the source term $S$, both analytic and/or numerical (finite differencing) solutions of Eqs. (5.21 - 5.24) are obtained and the results are compared with solutions obtained via the variational formulation. First, solutions to a single transport equation are investigated under various forms of the phenomenological coefficients $\kappa$, and $v$. Next, the variational proceedure is generalized to include several dependent variables and solutions to a coupled set of equations is presented.

The values of the various parameters used in this section are given in arbitrary units since we are interested only in comparing the performance of the variational formulation, and not in extracting specific physical information from the results.

\subsubsection{Linear Problem}

By assuming $\kappa=\kappa_{0}=$ const., $v=0$, and $S=\lambda T$ (where $\lambda=$ const.), the analytic solution of Eqs. $(5.21-5.24)$ is obtained in terms of the Bessel functions

$$
T(\rho, t)=2 \sum_{j=1}^{\infty} \frac{J_{0}\left(\nu_{j} \rho\right)}{\left[J_{1}\left(\nu_{j}\right)\right]^{2}} \exp \left[\left(\lambda-\nu_{j}^{2} \kappa_{0}\right) t\right] \int_{0}^{1} \rho f(\rho) J_{0}\left(\nu_{j} \rho\right) d \rho
$$

where $J_{p}$ is the Bessel function of order $p$, and where $\nu_{J}$ are the zeros of $J_{0}$ i.e. $J_{0}\left(\nu_{1}\right)=0$.

In comparing the variational with the analytic results, both the time behavior of the peak temperature, $T_{0}$, and the profile shapes will be considered. For $\kappa=0.5$, and $\lambda=1.0$ the time evolution of the peak temperature $T_{0}$, and the profile shapes at time $t=0.5$ are shown in Fig. 5.3. Note that both the time evolution of the peak temperature, and the profiles obtained by the analytic and variational methods are in excellent agreement. 

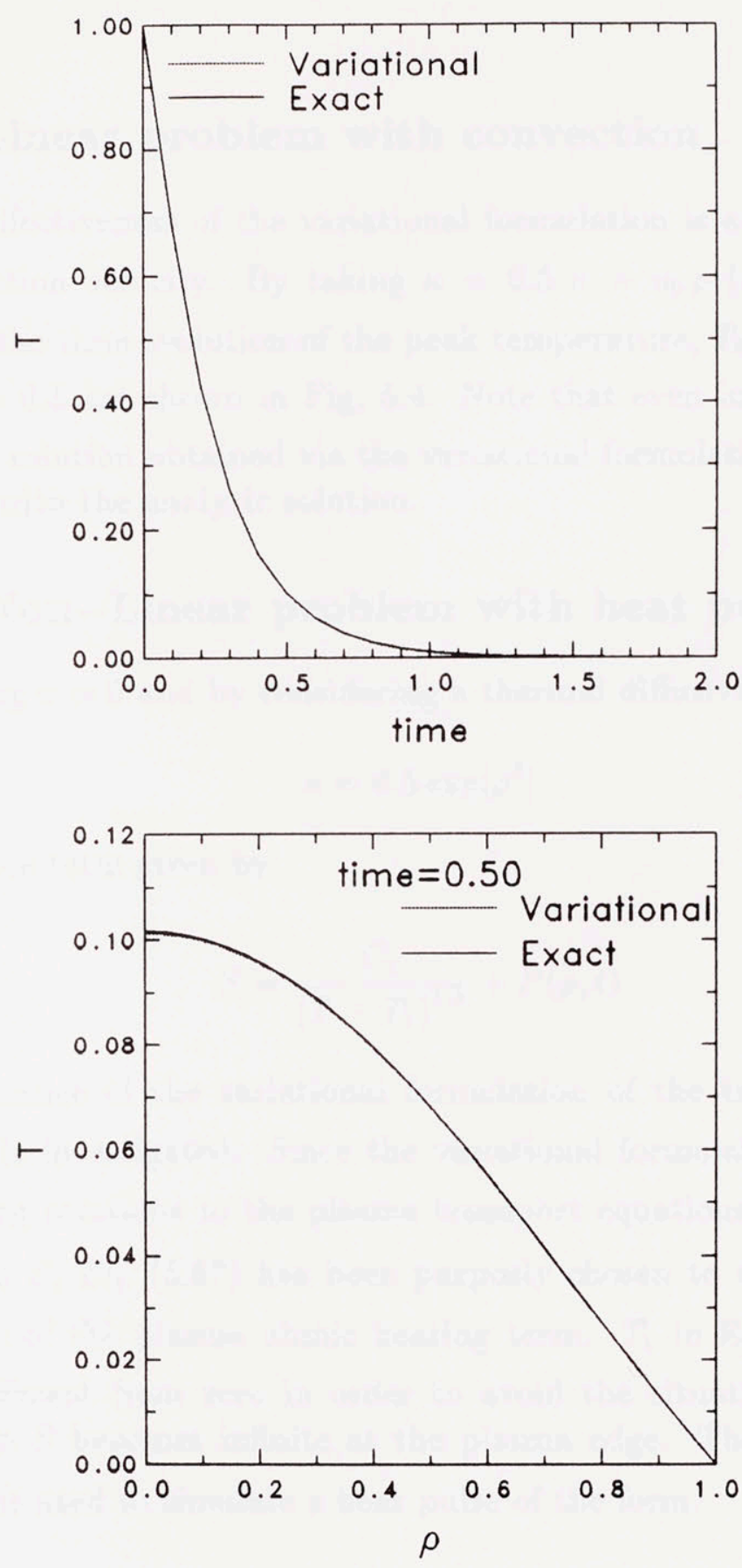

Figure 5.3: Comparison of the results obtained from the variational and the exact solution of a linear problem. Note that the time evolution of the peak temperature, shown in (a), is in excellent agreement for the two methods. In (b) the profiles are compared at time $t=0.5$ 


\subsubsection{Linear problem with convection}

Here, the effectiveness of the variational formulation is evaluated for nonzero convection velocity. By taking $\kappa=0.5 v=v_{0} \rho$ (where $v_{0}=0.5$ ), and $S=0$ the time evolution of the peak temperature, $T_{0}$, and the profiles at time $t=0.5$ are shown in Fig. 5.4. Note that even in the case of convection the solution obtained via the variational formulation is in excellent agreement with the analytic solution.

\subsubsection{Non-Linear problem with heat pulse}

By assuming $v=0$ and by considering a thermal diffusivity of the form

$$
\kappa=0.5 \exp \left[\rho^{2}\right]
$$

with a source term given by

$$
S=\frac{C_{1}}{\left(T+T_{1}\right)^{1.5}}+P(\rho, t)
$$

the performance of the variational formulation of the transport problem Eq. (5.21) is investigated. Since the variational formulation is developed for obtaining solutions to the plasma transport equations, the form of the source term $S$, Eq. (5.67) has been purposly chosen to model the $1 / T^{3 / 2}$ dependence of the plasma ohmic heating term. $T_{1}$ in Eq. (5.67) is some number different from zero in order to avoid the situation at which the source term $S$ becomes infinite at the plasma edge. The term $P(\rho, t)$ in Eq. (5.67) is used to simulate a heat pulse of the form.

$$
P=\left\{\begin{array}{cl}
0 & \text { if } t<t_{1} \\
P_{0} \exp \left[\frac{\left(\rho-\rho_{0}\right)^{2}}{\sigma^{2}}\right] & \text { if } t_{1} \leq t \leq t_{2} \\
0 & \text { if } t>t_{2}
\end{array}\right.
$$

In a physical situation, the term $P$ could represent the heating of a plasma due to externally applied RF or neutral beam heating. For $\rho_{0}=0, \sigma=0.5$, 

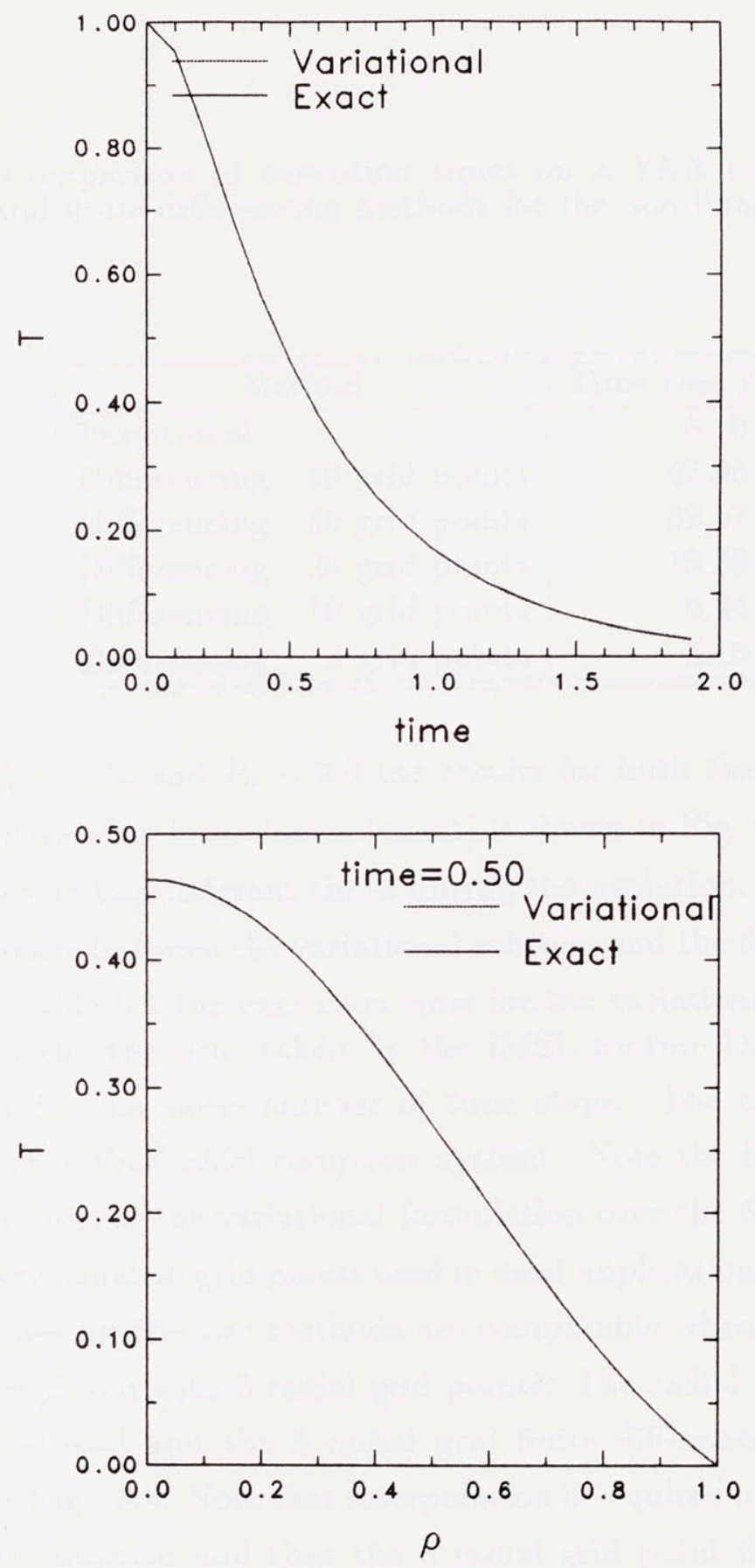

Figure 5.4: Comparison of the results obtained from the variational and the finite differencing formulation of a linear problem with convection. Note that the time evolution of the peak temperature, shown in (a), is in excellent agreement for the two methods. In (b) the profiles are compared at time $t=0.5$ 
Table 5.1: Comparison of execution times on a VAX computer for the variational and finite differencing methods for the non-linear problem with heat pulse

\begin{tabular}{|c|c|}
\hline Method & Time (sec.) \\
\hline Variational & 5.10 \\
\hline 40 grid points & 47.95 \\
\hline 30 grid points & 32.57 \\
\hline 20 grid points & 19.59 \\
\hline 10 grid points & 9.94 \\
\hline Differencing 5 grid points & 5.13 \\
\hline
\end{tabular}

$C_{1}=1.0, T_{1}=0.5$, and $P_{0}=2.0$ the results for both the variational and the finite differencing formulation (exact) is shown in Fig. 5.5. The profiles are compared at two different times during the evolution. Note that good agreement exists between the variational solution and the finite differencing solution. In table 5.1 the execution time for the variational formulation is compared to the the time taken by the IMSL routine DPDES to obtain the solution for the same number of time steps. The calculations were performed on a VAX 3800 computer system. Note the factor of 10 gain in computer time of the variational formulation over the finite differencing scheme for the usual 40 grid points used in most applications. Note also that the CPU times for the two methods are comparable when the differencing routine is employed with 5 radial grid points. The radial profiles obtained via the variational and the 5 radial grid finite differencing methods are compared in Fig. 5.6. Note that interpolation is required in order to extract the complete solution and thus the 5 radial grid point finite differencing method does not provide adequate resolution.

\subsubsection{Coupled equations.}

Since our goal is to apply the variational formulation to realistic plasma transport equations it is important to generalize the method to include 

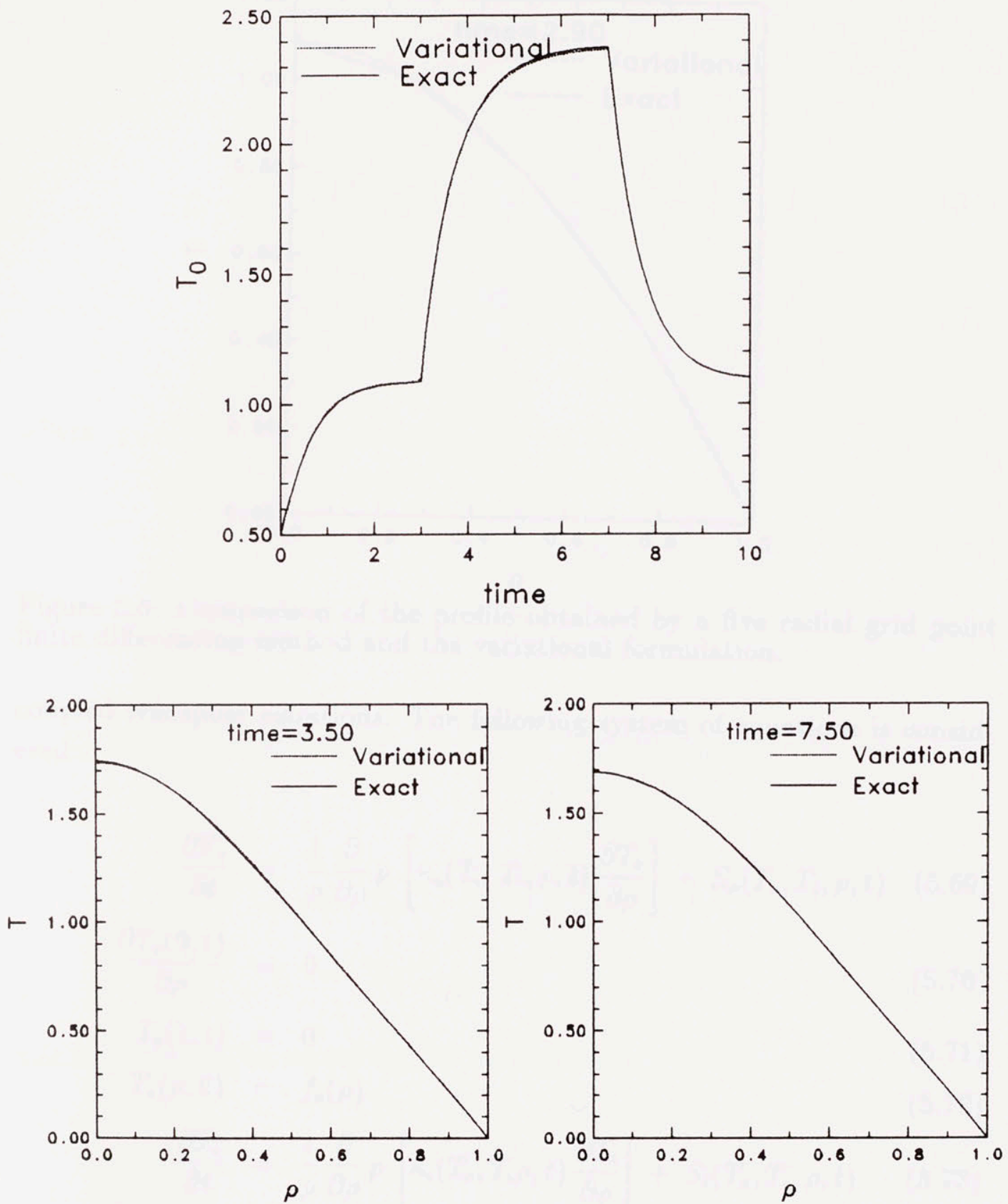

Figure 5.5: Comparison of the results obtained from the variational and the finite differencing formulation of the non-linear problem characterized by a centrally peaked heat pulse. Note that the time evolution of the peak temperature, shown in (a), is in excellent agreement for the two methods. In (b), and (c), the profiles are compared at times $t=3.5$ and $t=7.5$ respectively and there appears to be excellent agreement between the two methods. 


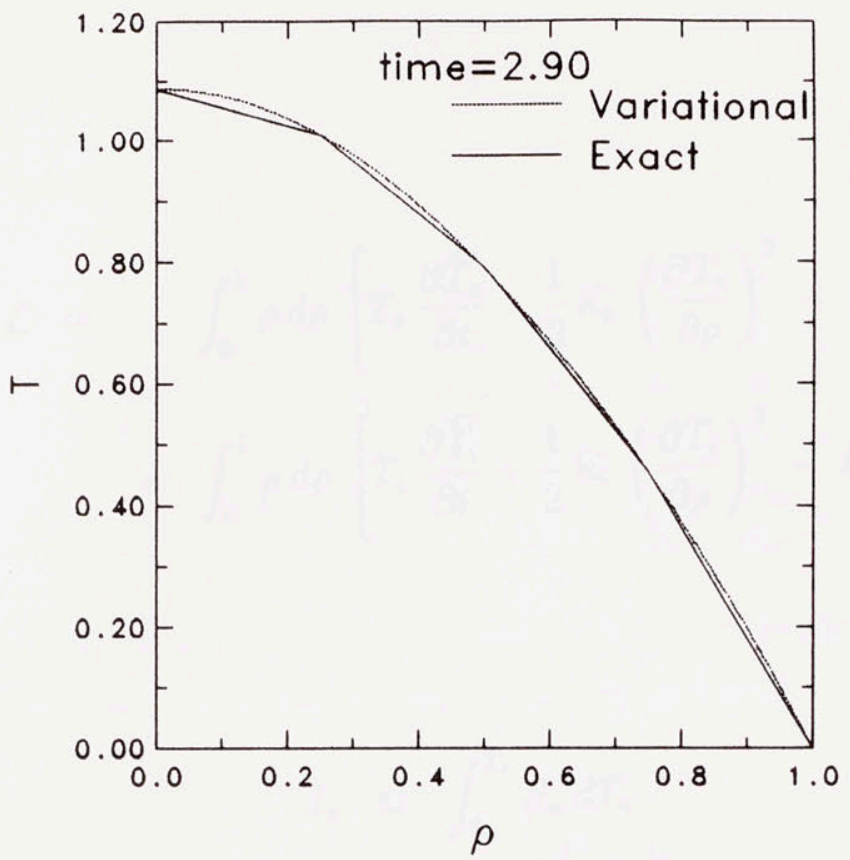

Figure 5.6: Comparison of the profile obtained by a five radial grid point finite differencing method and the variational formulation.

coupled transport equations. The following system of equations is considered

$$
\begin{aligned}
\frac{\partial T_{e}}{\partial t} & =\frac{1}{\rho} \frac{\partial}{\partial \rho} \rho\left[\kappa_{e}\left(T_{e}, T_{i}, \rho, t\right) \frac{\partial T_{e}}{\partial \rho}\right]+S_{e}\left(T_{e}, T_{i}, \rho, t\right) \\
\frac{\partial T_{e}(0, t)}{\partial \rho} & =0 \\
T_{e}(1, t) & =0 \\
T_{e}(\rho, 0) & =f_{e}(\rho) \\
\frac{\partial T_{i}}{\partial t} & =\frac{1}{\rho} \frac{\partial}{\partial \rho} \rho\left[\kappa_{i}\left(T_{e}, T_{i} \rho, t\right) \frac{\partial T_{i}}{\partial \rho}\right]+S_{i}\left(T_{e}, T_{i}, \rho, t\right) \\
\frac{\partial T_{i}(0, t)}{\partial \rho} & =0 \\
T_{i}(1, t) & =0 \\
T_{i}(\rho, 0) & =f_{i}(\rho)
\end{aligned}
$$

Following the formalism presented in section 5.3 the Lagrangian of the above system of equations is 


$$
\begin{aligned}
\mathcal{L}= & \int_{0}^{1} \rho d \rho\left[T_{e} \frac{\partial \widehat{T_{e}}}{\partial t}+\frac{1}{2} \widehat{\kappa_{e}}\left(\frac{\partial T_{e}}{\partial \rho}\right)^{2}-I_{e}\right] \\
+ & \int_{0}^{1} \rho d \rho\left[T_{i} \frac{\partial \widehat{T_{i}}}{\partial t}+\frac{1}{2} \widehat{\kappa_{i}}\left(\frac{\partial T_{i}}{\partial \rho}\right)^{2}-I_{i}\right]
\end{aligned}
$$

where

$$
\begin{aligned}
I_{e} & \equiv \int_{\widehat{T}_{e}}^{T_{e}} S_{e} d T_{e} \\
I_{i} & \equiv \int_{\widehat{T}_{i}}^{T_{i}} S_{i} d T_{i}
\end{aligned}
$$

By assuming trial functions of the form given by Eq. (5.33) for $T_{e}$, and $T_{i}$

$$
\begin{aligned}
& T_{e}=T_{e 0}\left(1-\rho^{2}\right) \exp \left[\alpha_{1} \rho^{2}+\alpha_{2} \rho^{4}\right] \\
& T_{i}=T_{i 0}\left(1-\rho^{2}\right) \exp \left[\beta_{1} \rho^{2}+\beta_{2} \rho^{4}\right]
\end{aligned}
$$

the equations to be solved can be written in matrix form

$$
\overrightarrow{\dot{V}}=\stackrel{\leftrightarrow}{M}^{-1} \vec{X}
$$

where $\vec{V}$ is a column vector with components

$$
\overrightarrow{\dot{V}}=\left[\begin{array}{c}
\dot{T}_{e 0} \\
\dot{\alpha}_{1} \\
\dot{\alpha}_{2} \\
\dot{T}_{i 0} \\
\dot{\beta}_{1} \\
\dot{\beta}_{2}
\end{array}\right]
$$


$\overleftrightarrow{\mathrm{M}}$ is a $6 \times 6$ matrix,

$$
\overleftrightarrow{\mathrm{M}}=\left[\begin{array}{cccccc}
A_{11} & A_{12} & A_{13} & 0 & 0 & 0 \\
A_{12} & A_{22} & A_{23} & 0 & 0 & 0 \\
A_{13} & A_{23} & A_{33} & 0 & 0 & 0 \\
0 & 0 & 0 & B_{11} & B_{12} & B_{13} \\
0 & 0 & 0 & B_{12} & B_{22} & B_{23} \\
0 & 0 & 0 & B_{13} & B_{23} & B_{33}
\end{array}\right]
$$

In the above matrix the elements labeled $A_{i j}$ correspond to $T_{e}$, and the elements labeled $B_{i j}$ correspond to $T_{i}$, and are given by

$$
\begin{aligned}
& A_{11}=\frac{1}{T_{e 0}^{2}} \int_{0}^{1} T_{e}^{2} \rho d \rho \\
& A_{12}=\frac{1}{T_{e 0}} \int_{0}^{1} T_{e}^{2} \rho^{3} d \rho \\
& A_{13}=\frac{1}{T_{e 0}} \int_{0}^{1} T_{e}^{2} \rho^{5} d \rho \\
& A_{22}=\int_{0}^{1} T_{e}^{2} \rho^{5} d \rho \\
& A_{23}=\int_{0}^{1} T_{e}^{2} \rho^{7} d \rho \\
& A_{33}=\int_{0}^{1} T_{e}^{2} \rho^{9} d \rho \\
& B_{11}=\frac{1}{T_{i 0}^{2}} \int_{0}^{1} T_{i}^{2} \rho d \rho \\
& B_{12}=\frac{1}{T_{i 0}} \int_{0}^{1} T_{i}^{2} \rho^{3} d \rho \\
& B_{13}=\frac{1}{T_{i 0}} \int_{0}^{1} T_{i}^{2} \rho^{5} d \rho \\
& B_{22}=\int_{0}^{1} T_{i}^{2} \rho^{5} d \rho \\
& B_{23}=\int_{0}^{1} T_{i}^{2} \rho^{7} d \rho
\end{aligned}
$$




$$
B_{33}=\int_{0}^{1} T_{i}^{2} \rho^{9} d \rho
$$

The elements of the column vector $\vec{X}$ are

$$
\begin{aligned}
& X_{1}=\frac{1}{T_{e 0}} \int_{0}^{1}\left[-\kappa_{e}\left(\frac{\partial T_{e}}{\partial \rho}\right)^{2}+S_{e} T_{e}\right] \rho d \rho \\
& X_{2}=\int_{0}^{1}\left[-\kappa_{e} \frac{\partial T_{e}}{\partial \rho} \frac{\partial}{\partial \rho}\left(\rho^{2} T_{e}\right)+S_{e} T_{e} \rho^{2}\right] \rho d \rho \\
& X_{3}=\int_{0}^{1}\left[-\kappa_{e} \frac{\partial T_{e}}{\partial \rho} \frac{\partial}{\partial \rho}\left(\rho^{4} T_{e}\right)+S_{e} T_{e} \rho^{4}\right] \rho d \rho \\
& X_{4}=\frac{1}{T_{i 0}} \int_{0}^{1}\left[-\kappa_{i}\left(\frac{\partial T_{i}}{\partial \rho}\right)^{2}+S_{i} T_{i}\right] \rho d \rho \\
& X_{5}=\int_{0}^{1}\left[-\kappa_{e} \frac{\partial T_{i}}{\partial \rho} \frac{\partial}{\partial \rho}\left(\rho^{2} T_{i}\right)+S_{i} T_{i} \rho^{2}\right] \rho d \rho \\
& X_{6}=\int_{0}^{1}\left[-\kappa_{e} \frac{\partial T_{i}}{\partial \rho} \frac{\partial}{\partial \rho}\left(\rho^{4} T_{i}\right)+S_{i} T_{i} \rho^{4}\right] \rho d \rho
\end{aligned}
$$

By assuming

$$
\begin{aligned}
\kappa_{e} & =0.1 \exp \left[\rho^{2}\right] \\
\kappa_{i} & =0.5 \exp \left[\rho^{2}\right] \\
S_{e} & =\frac{1}{\left(T_{e}+2.0\right)^{1.5}}+5.0 \frac{T_{i}-T_{e}}{\left(T_{e}+2.0\right)^{1.5}} \\
S_{i} & =-5.0 \frac{T_{i}-T_{e}}{\left(T_{e}+2.0\right)^{1.5}}
\end{aligned}
$$

the variational solution of Eqs. $(5.69,5.73)$ is compared, in Fig. 5.7, to the "exact" solution obtained by finite differencing. The time evolution of 
Table 5.2: Comparison of execution times on a VAX computer for the variational and finite differencing methods for the problem of coupled nonlineal transport equations.

\begin{tabular}{|c|c|}
\hline Method & Time (sec.) \\
\hline Variational & 5.71 \\
\hline 40 grid points & 39.27 \\
\hline 30 grid points & 28.16 \\
\hline 20 grid points & 21.37 \\
\hline 10 grid points & 9.18 \\
\hline Differencing $\quad 5$ grid points & 6.06 \\
\hline
\end{tabular}

the central values of $T_{e}$, and $T_{i}$ is shown in Fig. 5.7 (a), (b) respectively. Comparisons of the profiles for $T_{e}$, and $T_{i}$ at time $t=1.0$ are shown in Fig 5.7 (c), and (d). Table 5.2 shows approximate execution times, on a VAX 3800 computer system, for the variational formulation and for the finite differencing method.

\subsection{Variational Formulation of a General System of Transport Equations.}

Having developed and tested the variational formulation for a single transport equation, and for a system of two coupled transport equations the generalization of the formulation to an arbitrary number of transport equations is presented below.

The general one dimensional transport equation in cylindrical geometry is given by

$$
c_{j} \frac{\partial U_{j}}{\partial t}=\sum_{k} \frac{1}{\rho} \frac{\partial}{\partial \rho}\left(\rho D_{j k} \frac{\partial U_{k}}{\partial \rho}\right)+S_{j}
$$

where $U_{j}$ are the thermodynamic variables such as temperature, density, flux, etc. The parameters $c_{j}$ are various weight factors, $D_{j k}$ are the transport coefficients, and $S_{j}$ are the sources and sinks. 

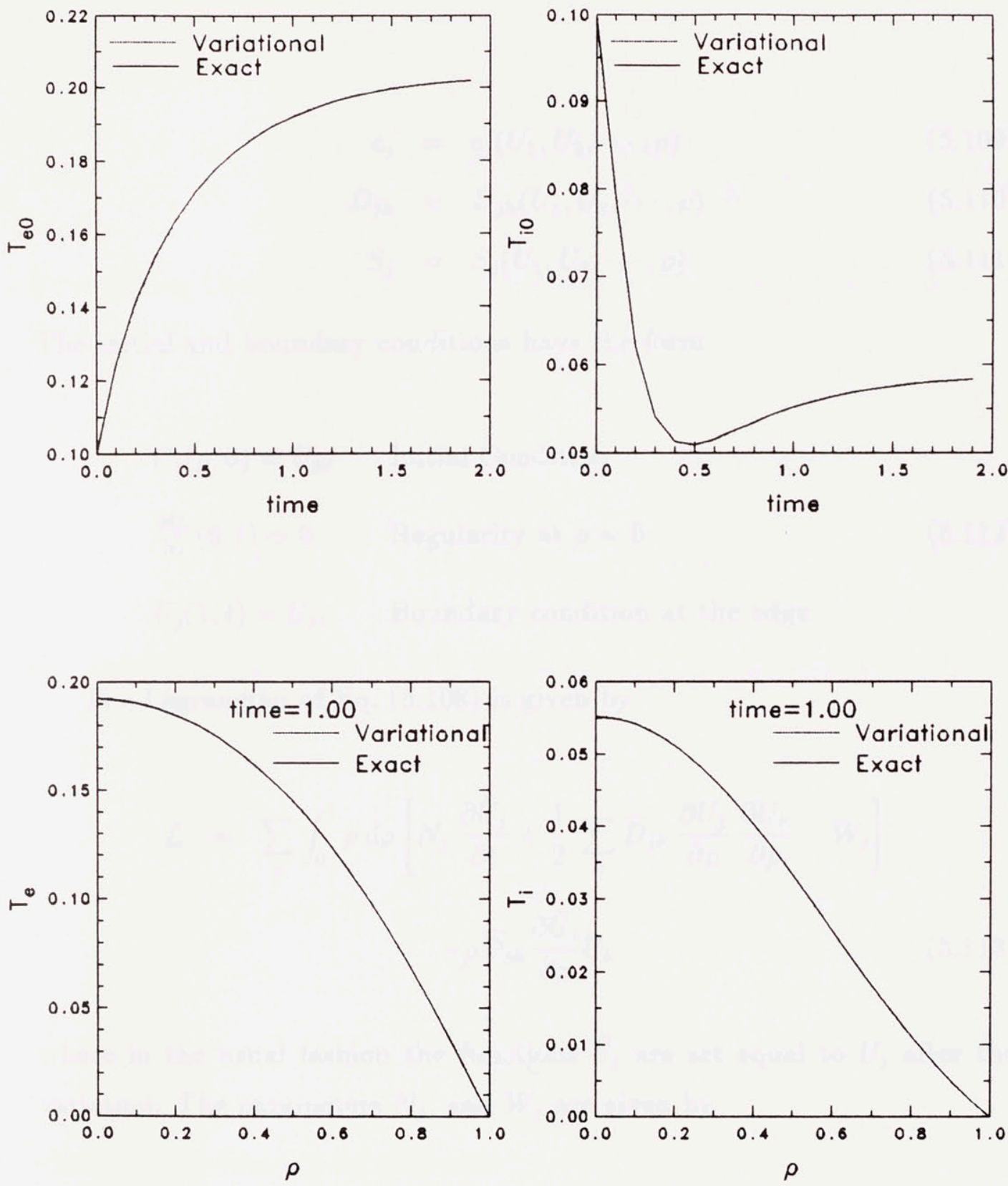

Figure 5.7: Comparison of the results obtained from the variational and the finite differencing formulation of a system of two coupled non-linear heat equations. The time evolution of the temperature for the two species is shown in (a), and (b). In (c), and (d) the profiles at time $t=1.0$ for the two species are compared. 


$$
\begin{aligned}
c_{j} & =c_{j}\left(U_{1}, U_{2}, \cdots, \rho\right) \\
D_{j k} & =D_{j k}\left(U_{1}, U_{2}, \cdots, \rho\right) \\
S_{j} & =S_{j}\left(U_{1}, U_{2}, \cdots, \rho\right)
\end{aligned}
$$

The initial and boundary conditions have the form

$$
\begin{array}{ll}
U_{j}(\rho, 0)=U_{j 0} & \text { Initial Condition } \\
\frac{\partial U_{i}}{\partial \rho}(0, t)=0 & \text { Regularity at } \rho=0 \\
U_{j}(1, t)=U_{j 1} & \text { Boundary condition at the edge }
\end{array}
$$

The Lagrangian of Eq. (5.108) is given by

$$
\begin{gathered}
\mathcal{L}=\sum_{j} \int_{0}^{1} \rho d \rho\left[N_{j} \frac{\partial \widehat{U}_{j}}{\partial t}+\frac{1}{2} \sum_{k} \widehat{D}_{j k} \frac{\partial U_{j}}{\partial \rho} \frac{\partial U_{k}}{\partial \rho}-W_{j}\right] \\
-\rho \widehat{D}_{j k} \frac{\partial \widehat{U}_{j}}{\partial \rho} U_{k}
\end{gathered}
$$

where in the usual fashion the functions $\widehat{U}_{j}$ are set equal to $U_{j}$ after the variation. The parameters $N_{j}$, and $W_{j}$ are given by

$$
\begin{aligned}
N_{j} & =N_{j}\left(\widehat{U}_{1}, \widehat{U}_{2}, \cdots, \widehat{U}_{j-1}, U_{j}, \widehat{U}_{j+1}, \cdots, \rho\right) \\
W_{j} & =W_{j}\left(\widehat{U}_{1}, \widehat{U}_{2}, \cdots, \widehat{U}_{j-1}, U_{j}, \widehat{U}_{j+1}, \cdots, \rho\right)
\end{aligned}
$$

Note that only $U_{j}$ is to be varied in Eqs. $(5.114,5.115)$. By taking the first variation of Eq. (5.113), and setting it equal to zero, the $j^{\text {th }}$ component becomes 


$$
\frac{\partial N_{j}}{\partial U_{j}} \frac{\partial U_{j}}{\partial t}-\sum_{k} \frac{1}{\rho} \frac{\partial}{\partial \rho} \rho\left(\frac{\widehat{D}_{j k}+\widehat{D}_{k j}}{2}\right) \frac{\partial U_{k}}{\partial \rho}-\frac{\partial W_{j}}{\partial \rho}=0
$$

By requiring Onsager symmetry for the matrix $D$ (i.e. $D_{j k}=D_{k j}$ ), and by defining

$$
\begin{array}{rlr}
N_{j} & =\int_{\widehat{U}_{j}}^{U_{j}} c_{j}\left(\widehat{\mathbf{U}}_{j^{\prime}} U_{j}, \rho\right) d U_{j} & j^{\prime} \neq j \\
W_{j}=\int_{\widehat{U}_{j}}^{U_{j}} S_{j}\left(\widehat{\mathbf{U}}_{j^{\prime}} U_{j}, \rho\right) d U_{j} & j^{\prime} \neq j
\end{array}
$$

the first variation of Eq. (5.113) yields the original equation as its Euler equation.

The Lagrangian can now be written in the matrix form

$$
\begin{gathered}
\mathcal{L}=\int_{0}^{1} \rho d \rho\left[\vec{N} \cdot \frac{\partial \hat{\vec{U}}}{\partial t}+\frac{1}{2}\left(\frac{\partial \vec{U}}{\partial \rho}\right)^{T} \cdot \widehat{\overleftrightarrow{D}} \cdot\left(\frac{\partial \vec{U}}{\partial \rho}\right)-\vec{W} \cdot \vec{I}\right] \\
-\left.\rho\left(\frac{\partial \vec{U}}{\partial \rho}\right)^{T} \cdot \widehat{\overleftrightarrow{D}} \cdot \vec{U}\right|_{\rho}=1
\end{gathered}
$$

where $\vec{I}$ is the identity column vector.

\subsection{Conclusion}

The method by which variational techniques can be used to obtain solutions to the transport equations has been developed in this chapter. The accuracy of the variational formulationa has been investigated in the cases for which analytic solutions exist. Also, the variational formulation was tested 
for accuracy and speed of execution for a single non-linear equation and for a problem of coupled non-linear equations. It is shown that accurate solutions can be obtained by employing the variational formulation with a decrease by an order of magnitude in the required computer execution time. Further reduction in the execution time can be obtained by optimizing the computation. This optimization can be obtained by precalculating certain frequently used quantities and integrals, as well as by optimizing the matrix inversion algorithm. 


\section{Chapter 6}

\section{Variational Solutions of the Plasma Transport Equations}

\subsection{Introduction}

Since the first successful measurements of the spatial profiles for the electron density and temperature in the T-3 Tokamak [56] many computer models have been developed for predicting and understanding Tokamak plasma transport. Presently, some of the most widely used Tokamak transport computer models are: TSC [57], developed at Princeton University, BALDUR [58], also developed at Princeton, WHIST [59], developed at Oak Ridge National Laboratory, and TRANSP [60]. Furthermore, more codes are developed at other laboratories mainly for the purpose of addressing specific transport questions.

In general, a Tokamak transport model can be characterized as Predictive [61], Comparative or both. A Predictive model is based on existing plasma theory, and it is used in order to predict the behavior of machines during their design stage or to predict the effect of a certain mode of operation (auxiliary heating, fueling, alpha particle effects, etc.) during machine operation. Such a model can also serve as a test of the underlying plasma theory by comparing its predictions to existing experimental data. A Comparative tokamak computer model is one whose primary goal is to compare various theoretical or empirical transport models to the experimental data. 
Developing a complete transport model and implementing it on the computer requires extensive effort. For this reason scientists wishing to perform transport calculations resort to one of the transport models mentioned above. These models are based on standard computational schemes and provide good spatial and temporal resolution. However, they are very computationally intensive requiring long computer time for evolving the solution.

On chapter 2 various plasma transport models were developed. The volume averaged (0-D) model was in turn used in chapter 3 and chapter 4 in order to investigate ignition and burn control characteristics of tokamaks. The 0 -D model is in general easy to develop and use. The computer time required for obtaining results from a 0 -D model is very short enabling parametric studies to be performed. However a 0 -D model has many limitations with the primary problem being the inability to investigate profile effects. Ideally therefore it is desirable to obtain a plasma transport model which incorporates the profile effects which are absent from the 0-D formulation and which is fast to execute on a computer.

As it is shown in chapter 5 the variational formalism can be used in order to solve the transport equations with a significant savings in computation time, while retaining high accuracy. Therefore by using the formulation developed on chapter 5 it is possible to develop a complete plasma transport model which incorporates the desirable aspects of both the 0 -D and the standard 1-D formalisms. In particular such a model gives accurate results very fast.

In this chapter a complete variational plasma transport model is developed.

\subsection{Variational Formulation of the Plasma Transport Equations}

The variational techniques developed and tested in chapter 5 are now used in order to obtain solutions to the plasma transport equations. The equations 
to be formulated variationally and solved is the set deduced from the fast equilibration approximation Eqs. (2.153 - 2.157, and 2.159). The equations are again listed here for convenience.

$$
\begin{aligned}
\frac{\partial n_{d}}{\partial t}= & \frac{1}{V^{\prime}} \frac{\partial}{\partial \rho} V^{\prime}\left(D_{d} \frac{\partial n_{d}}{\partial \rho}+v_{d} n_{d}\right)+\left\langle S_{n d}\right\rangle \\
\frac{\partial n_{t}}{\partial t}= & -\frac{1}{V^{\prime}} \frac{\partial}{\partial \rho} V^{\prime}\left(D_{t} \frac{\partial n_{t}}{\partial \rho}+v_{t} n_{t}\right)+\left\langle S_{n t}\right\rangle \\
\frac{\partial n_{\alpha}}{\partial t}= & -\frac{1}{V^{\prime}} \frac{\partial}{\partial \rho} V^{\prime}\left(D_{\alpha} \frac{\partial n_{\alpha}}{\partial \rho}+v_{\alpha} n_{\alpha}\right)+\left\langle S_{n \alpha}\right\rangle \\
\frac{\partial n_{i m p}}{\partial t}= & -\frac{1}{V^{\prime}} \frac{\partial}{\partial \rho} V^{\prime}\left(D_{i m p} \frac{\partial n_{i m p}}{\partial \rho}+v_{i m p} n_{i m p}\right)+\left\langle S_{n(i m p)}\right\rangle \\
\frac{3}{2} \frac{\partial}{\partial t}\left(n_{e}+n_{i}\right) T= & \frac{1}{V^{\prime}} \frac{\partial}{\partial \rho}\left[V^{\prime}\left(\left(n_{e} \chi_{e}+n_{i} \chi_{i}\right) \frac{\partial T}{\partial \rho}+\frac{5}{2} T \Gamma\right)\right] \\
& +\left\langle S_{E e}\right\rangle+\left\langle S_{E i}\right\rangle \\
T_{e}= & T+T_{1} \\
\frac{\partial \psi}{\partial t}= & \frac{F(\rho)}{\left\langle 1 / R^{2}\right\rangle} \frac{\eta_{\|}}{V^{\prime}(\rho)} \frac{\partial}{\partial \rho}\left[\frac{V^{\prime}(\rho)}{F(\rho)}\left\langle\frac{|\nabla \rho|^{2}}{R^{2}}\right\rangle \frac{\partial \psi}{\partial \rho}\right] \\
& +\frac{\left\langle\vec{E}_{e x t} \cdot \vec{B}\right\rangle}{\left\langle 1 / R^{2}\right\rangle F(\rho)} \\
n_{e}= & n_{d}+n_{t}+2 n_{\alpha}+Z_{i m p} n_{i m p} \\
n_{i}= & n_{d}+n_{t}+n_{\alpha}+n_{i m p}
\end{aligned}
$$

Where $\langle Q\rangle$ denotes the flux surface average of $Q$, and where the $T_{1}$ is given by Eq. (2.152). The initial and boundary conditions for the above equations may be written in the general form

$$
\begin{array}{ll}
U(\rho, 0)=U_{0}(\rho) & \text { Initial Condition } \\
U(0, t)=\text { regular } & \text { Boundary Condition at the Origin } \\
U(1, t)=U_{1}(t) & \text { Boundary Condition at the Edge }
\end{array}
$$


where $U$ can be any of the dependent variables being evolved (i.e. $U=$ $\left.n_{d}, n_{t}, n_{\alpha}, n_{i m p}, T, \psi\right)$.

\subsubsection{Lagrangian Formulation}

Following the procedure developed in chapter 5 the Lagrangian of Eqs. (6.1

- 6.5, and 6.7) may be written as

$$
\begin{aligned}
\mathcal{L} & =\int_{0}^{1} V^{\prime} d \rho\left[\vec{N}^{T} \cdot \frac{\partial \hat{\vec{U}}}{\partial t}+\frac{1}{2}\left(\frac{\partial \vec{U}}{\partial \rho}\right)^{T} \cdot \widehat{\vec{D}} \cdot\left(\frac{\partial \vec{U}}{\partial \rho}\right)-\vec{I}^{T} \cdot \hat{\vec{\Gamma}} \cdot\left(\frac{\partial \vec{U}}{\partial \rho}\right)-\vec{W}^{T} \cdot \vec{I}\right] \\
& -\left.\underbrace{V^{\prime}\left(\frac{\partial \widehat{\vec{U}}}{\partial \rho}\right)^{T} \cdot \widehat{\vec{D}} \cdot \vec{U}}_{S_{1}}\right|_{\rho=1}+\left.\underbrace{V^{\prime} \widehat{\vec{U}}^{T} \cdot \widehat{\vec{\Gamma}} \cdot \vec{U}}_{S_{2}}\right|_{\rho=1}
\end{aligned}
$$

Note that in this variational principle the last two terms contain the "natural boundary conditions" of the system. [4] This formulation allows for trial functions to be substituted whose variation does not vanish at the plasma edge (see section 5.4 for a discussion of this issue).

The vector $\vec{N}$ is given by

$$
\vec{N} \equiv\left[\begin{array}{c}
n_{d} \\
n_{t} \\
n_{\alpha} \\
n_{i m p} \\
\frac{3}{2}\left(\widehat{n}_{e}+\widehat{n}_{i}\right) T \\
\psi
\end{array}\right]
$$

The column vectors $\partial \vec{U} / \partial t$ and $\partial \vec{U} / \partial \rho$ denote the time and space derivative of $\vec{U}$ and are given by 


$$
\begin{aligned}
& \frac{\partial \vec{U}}{\partial t} \equiv\left[\begin{array}{c}
\frac{\partial n_{d}}{\partial t} \\
\frac{\partial n_{t}}{\partial t} \\
\frac{\partial n_{\alpha}}{\partial t} \\
\frac{\partial n_{i m p}}{\partial t} \\
\frac{\partial T}{\partial t} \\
\frac{\partial \psi}{\partial t}
\end{array}\right] \\
& \frac{\partial \vec{U}}{\partial \rho} \equiv\left[\begin{array}{c}
\frac{\partial n_{d}}{\partial \rho} \\
\frac{\partial n_{t}}{\partial \rho} \\
\frac{\partial n_{\alpha}}{\partial \rho} \\
\frac{\partial n_{i m p}}{\partial \rho} \\
\frac{\partial T}{\partial \rho} \\
\frac{\partial \psi}{\partial \rho}
\end{array}\right]
\end{aligned}
$$

The matrix $\stackrel{\leftrightarrow}{\mathrm{D}}$ is diagonal and is given by

$$
\overleftrightarrow{\mathrm{D}}=\left[\begin{array}{cccccc}
D_{d} & 0 & 0 & 0 & 0 & 0 \\
0 & D_{t} & 0 & 0 & 0 & 0 \\
0 & 0 & D_{\alpha} & 0 & 0 & 0 \\
0 & 0 & 0 & D_{i m p} & 0 & 0 \\
0 & 0 & 0 & 0 & n_{e} \chi_{e}+n_{i} \chi_{i} & 0 \\
0 & 0 & 0 & 0 & 0 & D_{\psi}
\end{array}\right]
$$

where $D_{\psi}=\eta\left\langle|\nabla \rho|^{2} / R^{2}\right\rangle / F(\rho) . \quad \vec{I}$ is simply a vector whose elements are equal to one (unit column vector).

The third term on the right hand side of Eq. (6.11) represents the various convective terms of the transport equations. The convective particle fluxes $\Gamma_{j}$ are included as the diagonal elements of the matrix $\stackrel{\leftrightarrow}{\Gamma}$ 


$$
\overleftrightarrow{\Gamma}=\left[\begin{array}{cccccc}
v_{d} n_{d} & 0 & 0 & 0 & 0 & 0 \\
0 & v_{t} n_{t} & 0 & 0 & 0 & 0 \\
0 & 0 & v_{\alpha} n_{\alpha} & 0 & 0 & 0 \\
0 & 0 & 0 & v_{i m p} n_{i m p} & 0 & 0 \\
0 & 0 & 0 & 0 & \frac{5}{2} \Gamma_{T} & 0 \\
0 & 0 & 0 & 0 & 0 & 0
\end{array}\right]
$$

here, $\Gamma_{T}$ represents the total convective heat flux and is given by

$$
\Gamma_{T}=\left(n_{e} v_{e}+n_{i} v_{i}\right) T
$$

The sources and sinks for energy and mass are included in the elements of the vector $\vec{W}$, which is given by

$$
\vec{W} \equiv\left[\begin{array}{c}
W_{1} \\
W_{2} \\
W_{3} \\
W_{4} \\
W_{5} \\
W_{6} \\
W_{7}
\end{array}\right]
$$

where the elements $W_{j}$ are of the form

$$
\begin{aligned}
& W_{1}=\int_{\widehat{n}_{d}}^{n_{d}}\left\langle S_{d}\right\rangle d n_{d} \\
& W_{2}=\int_{\widehat{n}_{t}}^{n_{t}}\left\langle S_{t}\right\rangle d n_{t} \\
& W_{3}=\int_{\widehat{n}_{\alpha}}^{n_{\alpha}}\left\langle S_{\alpha}\right\rangle d n_{\alpha} \\
& W_{4}=\int_{\widehat{n}_{i m p}}^{n_{i m p}}\left\langle S_{i m p}\right\rangle d n_{i m p} \\
& W_{5}=\int_{\widehat{T}}^{T}\left(\left\langle S_{E e}\right\rangle+\left\langle S_{E i}\right\rangle d n_{T}\right.
\end{aligned}
$$




$$
\begin{aligned}
& W_{6}=\frac{3}{4} T^{2} \frac{\partial}{\partial t}\left(n_{e}+n_{i}\right) \\
& W_{7}=\int_{\widehat{\psi}}^{\psi} \frac{\left\langle\vec{E}_{\text {ext }} \cdot \vec{B}\right\rangle}{\left\langle 1 / R^{2}\right\rangle F(\rho)} d \psi
\end{aligned}
$$

\subsubsection{Trial Functions}

Once the Lagrangian is determined the next fundamental problem in the variational formulation is the choice of an appropriate trial function. In general, a trial function must be capable of representing the actual solution of the equations to be solved, and it is desired that it must be of a simple mathematical form.

For a tokamak plasma the dependent variables $(n, T, \psi)$ may be accurately represented by a trial function of the form

$$
U=U_{0}\left(1-\gamma \rho^{2}\right) \exp \left[\alpha \rho^{2}+\beta \rho^{4}\right]
$$

The parameters $U_{0}, \alpha$, and $\beta$ (the variational parameters) characterize the overall shape of the profiles. In particular, $U_{0}$ determines the central value of $U$, and $\alpha, \beta$ (the shape parameters) characterize the profile shape. This type of trial function is very flexible and can represent a wide variety of profiles by suitably choosing the values of the shape parameters $\alpha$, and $\beta$. Its flexibility was investigated in chapter 5 and is shown in Fig 5.2. Since the variation $\delta U$ of the trial function given by Eq. (6.26) does not vanish at the plasma edge $(\rho=1)$, an exact variational formulation requires a Lagrangian of the form given by Eq. (6.11) which includes the natural boundary conditions.

The parameter $\gamma$ in Eq. (6.26) is related to the outer, $(\rho=1)$, boundary condition. In general with this type of trial function it is possible to model both fixed and floating edge values. In this analysis, a floating edge value is one which is related to the evolving variables. For example, a simple model with floating edge values is one which requires that the edge value 
is some fraction of the central value. Also, as a floating edge value can be characterized the case which determines the boundary conditions from some kind of plasma edge model.

For the case of floating edge values $\left(U(1, t)=U_{1}(t)\right)$ the parameter $\gamma$ becomes

$$
\gamma=1-\frac{U_{1}(t)}{U_{0} \exp [\alpha+\beta]}
$$

Similarly, for for fixed edge values $\gamma$ becomes

$$
\gamma=1-\frac{U_{1}}{U_{0} \exp [\alpha+\beta]}
$$

Another trial function, similar to the one given by Eq. (6.26), is

$$
U=\left(U_{0}-U_{\text {edge }}\right)\left(1-\rho^{2}\right) \exp \left[\alpha_{1} \rho^{2}+\alpha_{2} \rho^{4}\right]+U_{\text {edge }}
$$

Where $U_{\text {edge }}$ corresponds to the value of $U$ at $\rho=1$. With this trial function the variation $\delta U$ at the plasma edge is zero and thus the appropriate Lagrangian is given by Eq. (6.11) without the last two surface terms labeled $S_{1}$, and $S_{2}$.

The two trial functions represented by Eqs. $(6.26,6.29)$, when used with the appropriate Lagrangians, are equivalent in their effectiveness of representing the solution. The formulation presented in this analysis assumes trial functions of the form given by Eq. (6.26) with the Lagrangian given by Eq. (6.11). This choice allows for a more compact presentation of the results to follow.

Explicitly, the trial functions used for the various thermodynamic variables are

$$
n_{d}=n_{d 0}\left(1-\gamma_{d} \rho^{2}\right) \exp \left[\alpha_{d} \rho^{2}+\beta_{d} \rho^{4}\right]
$$




$$
\begin{aligned}
n_{t} & =n_{t 0}\left(1-\gamma_{t} \rho^{2}\right) \exp \left[\alpha_{t} \rho^{2}+\beta_{t} \rho^{4}\right] \\
n_{\alpha} & =n_{\alpha 0}\left(1-\gamma_{\alpha} \rho^{2}\right) \exp \left[\alpha_{\alpha} \rho^{2}+\beta_{\alpha} \rho^{4}\right] \\
n_{i m p} & =n_{i m p 0}\left(1-\gamma_{i m p} \rho^{2}\right) \exp \left[\alpha_{i m p} \rho^{2}+\beta_{i m p} \rho^{4}\right] \\
T & =T_{0}\left(1-\gamma_{T} \rho^{2}\right) \exp \left[\alpha_{T} \rho^{2}+\beta_{T} \rho^{4}\right] \\
\psi & =\psi_{0}\left(1-\gamma_{\psi} \rho^{2}\right) \exp \left[\alpha_{\psi} \rho^{2}+\beta_{\psi} \rho^{4}\right]
\end{aligned}
$$

Similarly, the trial functions for the quantities accented with a (f) (i.e. the functions which are not subject to variations) are given by

$$
\begin{aligned}
\widehat{n}_{d} & =\widehat{n}_{d 0}\left(1-\gamma_{d} \rho^{2}\right) \exp \left[\widehat{\alpha}_{d} \rho^{2}+\widehat{\beta}_{d} \rho^{4}\right] \\
\widehat{n}_{t} & =\widehat{n}_{t 0}\left(1-\gamma_{t} \rho^{2}\right) \exp \left[\widehat{\alpha}_{t} \rho^{2}+\widehat{\beta}_{t} \rho^{4}\right] \\
\widehat{n}_{\alpha} & =\widehat{n}_{\alpha 0}\left(1-\gamma_{\alpha} \rho^{2}\right) \exp \left[\widehat{\alpha}_{\alpha} \rho^{2}+\widehat{\beta}_{\alpha} \rho^{4}\right] \\
\widehat{n}_{i m p} & =\widehat{n}_{i m p 0}\left(1-\gamma_{i m p} \rho^{2}\right) \exp \left[\widehat{\alpha}_{i m p} \rho^{2}+\widehat{\beta}_{i m p} \rho^{4}\right] \\
\widehat{T} & =\widehat{T}_{0}\left(1-\gamma_{T} \rho^{2}\right) \exp \left[\widehat{\alpha}_{T} \rho^{2}+\widehat{\beta}_{T} \rho^{4}\right] \\
\widehat{\psi} & =\widehat{\psi}_{0}\left(1-\gamma_{\psi} \rho^{2}\right) \exp \left[\widehat{\alpha}_{\psi} \rho^{2}+\widehat{\beta}_{\psi} \rho^{4}\right]
\end{aligned}
$$

In summary, the variational parameters are;

$\begin{array}{ll}n_{d 0}, \alpha_{d}, \beta_{d} & \text { For the Deuterium } \\ n_{t 0}, \alpha_{t}, \beta_{t} & \text { For the Tritium } \\ n_{\alpha 0}, \alpha_{\alpha}, \beta_{\alpha} & \text { For the Alpha Particles } \\ n_{i m p 0}, \alpha_{i m p}, \beta_{i m p} & \text { For the Impurities } \\ T_{0}, \alpha_{T}, \beta_{T} & \text { For the Temperature } \\ \psi_{0}, \alpha_{\psi}, \beta_{\psi} & \text { For the Magnetic Flux }\end{array}$




\subsubsection{System of ODE's to be Solved}

Having developed the Lagrangian for the equations to be solved and the trial functions which will represent the solution, the next step is to determine the values of the variational parameters which make the Lagrangian $\mathcal{L}$ stationary. As it has been shown in chapter 5 these values when substituted in the trial functions give the best possible solutions to the plasma transport equations. By using the convention that $\xi_{j}$ represents all the variational parameters, the stationary value of the Lagrangian is obtained by setting

$$
\frac{\delta \mathcal{L}\left(\xi_{j}, \widehat{\xi}_{j}\right)}{\delta \xi_{j}}=0
$$

subject to the subsidiary conditions

$$
\widehat{\xi}_{j}=\xi_{j}
$$

By performing the operation indicated by Eq. (6.43) for each of the variational parameters it is found that the problem can be put in the matrix form

$$
\overrightarrow{\dot{\xi}}=\stackrel{\mathrm{M}}{ }^{-1} \cdot \vec{R}
$$

where $\overrightarrow{\dot{\xi}}$ denotes the time derivative of the variational parameters 


$$
\vec{\xi} \equiv\left[\begin{array}{c}
\dot{n}_{d 0} \\
\dot{\alpha}_{d} \\
\dot{\beta}_{d} \\
\dot{n}_{t 0} \\
\dot{\alpha}_{t} \\
\dot{\beta}_{t} \\
\vdots \\
\dot{T}_{0} \\
\dot{\alpha}_{T} \\
\dot{\beta}_{T} \\
\dot{\psi}_{0} \\
\dot{\alpha}_{\psi} \\
\dot{\beta}_{\psi}
\end{array}\right]
$$

$\overleftrightarrow{\mathrm{M}}$ is a $18 \times 18$ codiagonal matrix which may be written in the compact form

$$
\overleftrightarrow{\mathbf{M}} \equiv\left[\begin{array}{cccccc}
\overleftrightarrow{\mathbf{A}}_{d} & {[0]} & {[0]} & {[0]} & {[0]} & {[0]} \\
{[0]} & \overleftrightarrow{\mathbf{A}}_{t} & {[0]} & {[0]} & {[0]} & {[0]} \\
{[0]} & {[0]} & \overleftrightarrow{\mathbf{A}}_{\alpha} & {[0]} & {[0]} & {[0]} \\
{[0]} & {[0]} & {[0]} & \overleftrightarrow{\mathbf{A}}_{i m p} & {[0]} & {[0]} \\
{[0]} & {[0]} & {[0]} & {[0]} & \overleftrightarrow{\mathbf{A}}_{T} & {[0]} \\
{[0]} & {[0]} & {[0]} & {[0]} & {[0]} & \overleftrightarrow{\mathbf{A}}_{\psi}
\end{array}\right]
$$

where each of the elements $\overleftrightarrow{\mathbf{A}}_{j}$ is a $3 \times 3$ symmetric matrix. The elements $[0]$ denote $3 \times 3$ matrices whose elements are zero.

The elements of $\overleftrightarrow{\mathbf{A}}_{d}$ are related to the deuterium equation and are given by

$$
\overleftrightarrow{\mathbf{A}_{d}} \equiv\left[\begin{array}{ccc}
A_{d 11} & A_{d 12} & A_{d 13} \\
A_{d 12} & A_{d 22} & A_{d 23} \\
A_{d 13} & A_{d 23} & A_{d 33}
\end{array}\right]
$$




$$
\begin{aligned}
& A_{d 11}=\frac{1}{n_{d 0}^{2}} \int_{0}^{1} V^{\prime} n_{d}^{2} d \rho \\
& A_{d 12}=\frac{1}{n_{d 0}} \int_{0}^{1} V^{\prime} \rho^{2} n_{d}^{2} d \rho \\
& A_{d 13}=\frac{1}{n_{d 0}} \int_{0}^{1} V^{\prime} \rho^{4} n_{d}^{2} d \rho \\
& A_{d 22}=\int_{0}^{1} V^{\prime} \rho^{4} n_{d}^{2} d \rho \\
& A_{d 23}=\int_{0}^{1} V^{\prime} \rho^{6} n_{d}^{2} d \rho \\
& A_{d 11}=\int_{0}^{1} V^{\prime} \rho^{8} n_{d}^{2} d \rho
\end{aligned}
$$

The elements of the matrix $\overleftrightarrow{\mathbf{A}}_{t}$ are related to the tritium equation and are given by

$$
\begin{aligned}
\overleftrightarrow{\mathbf{A}}_{t} & =\left[\begin{array}{lll}
A_{t 11} & A_{t 12} & A_{t 13} \\
A_{t 12} & A_{t 22} & A_{t 23} \\
A_{t 13} & A_{t 23} & A_{t 33}
\end{array}\right] \\
A_{t 11} & =\frac{1}{n_{t 0}^{2}} \int_{0}^{1} V^{\prime} n_{t}^{2} d \rho \\
A_{t 12} & =\frac{1}{n_{t 0}} \int_{0}^{1} V^{\prime} \rho^{2} n_{t}^{2} d \rho \\
A_{t 13} & =\frac{1}{n_{t 0}} \int_{0}^{1} V^{\prime} \rho^{4} n_{t}^{2} d \rho \\
A_{t 22} & =\int_{0}^{1} V^{\prime} \rho^{4} n_{t}^{2} d \rho \\
A_{t 23} & =\int_{0}^{1} V^{\prime} \rho^{6} n_{t}^{2} d \rho \\
A_{t 11} & =\int_{0}^{1} V^{\prime} \rho^{8} n_{t}^{2} d \rho
\end{aligned}
$$


The the matrix associated with the alpha particle equation is $\overleftrightarrow{\mathbf{A}}_{\alpha}$ and is given by

$$
\begin{aligned}
& \overleftrightarrow{\mathbf{A}}_{\alpha} \equiv\left[\begin{array}{lll}
A_{\alpha 11} & A_{\alpha 12} & A_{\alpha 13} \\
A_{\alpha 12} & A_{\alpha 22} & A_{\alpha 23} \\
A_{\alpha 13} & A_{\alpha 23} & A_{\alpha 33}
\end{array}\right] \\
& A_{\alpha 11}=\frac{1}{n_{\alpha 0}^{2}} \int_{0}^{1} V^{\prime} n_{\alpha}^{2} d \rho \\
& A_{\alpha 12}=\frac{1}{n_{\alpha 0}} \int_{0}^{1} V^{\prime} \rho^{2} n_{\alpha}^{2} d \rho \\
& A_{\alpha 13}=\frac{1}{n_{\alpha 0}} \int_{0}^{1} V^{\prime} \rho^{4} n_{\alpha}^{2} d \rho \\
& A_{\alpha 22}=\int_{0}^{1} V^{\prime} \rho^{4} n_{\alpha}^{2} d \rho \\
& A_{\alpha 23}=\int_{0}^{1} V^{\prime} \rho^{6} n_{\alpha}^{2} d \rho \\
& A_{\alpha 11}=\int_{0}^{1} V^{\prime} \rho^{8} n_{\alpha}^{2} d \rho
\end{aligned}
$$

Similarly the matrix $\overleftrightarrow{\mathbf{A}}_{\text {imp }}$ is associated with the impurity equation and is given by

$$
\begin{aligned}
& \overleftrightarrow{\mathbf{A}}_{i m p} \equiv\left[\begin{array}{lll}
A_{i m p 11} & A_{i m p 12} & A_{i m p 13} \\
A_{i m p 12} & A_{i m p 22} & A_{i m p 23} \\
A_{i m p 13} & A_{i m p 23} & A_{i m p 33}
\end{array}\right] \\
& A_{i m p 11}=\frac{1}{n_{i m p 0}^{2}} \int_{0}^{1} V^{\prime} n_{i m p}^{2} d \rho \\
& A_{i m p 12}=\frac{1}{n_{i m p 0}} \int_{0}^{1} V^{\prime} \rho^{2} n_{i m p}^{2} d \rho
\end{aligned}
$$




$$
\begin{aligned}
& A_{i m p 13}=\frac{1}{n_{i m p 0}} \int_{0}^{1} V^{\prime} \rho^{4} n_{i m p}^{2} d \rho \\
& A_{i m p 22}=\int_{0}^{1} V^{\prime} \rho^{4} n_{i m p}^{2} d \rho \\
& A_{i m p 23}=\int_{0}^{1} V^{\prime} \rho^{6} n_{i m p}^{2} d \rho \\
& A_{i m p 11}=\int_{0}^{1} V^{\prime} \rho^{8} n_{i m p}^{2} d \rho
\end{aligned}
$$

The temperature equation is represented by the matrix $\overleftrightarrow{\mathbf{A}}_{T}$ whose elements are given by

$$
\begin{aligned}
\stackrel{\mathbf{A}}{T}_{T} & \equiv\left[\begin{array}{lll}
A_{T 11} & A_{T 12} & A_{T 13} \\
A_{T 12} & A_{T 22} & A_{T 23} \\
A_{T 13} & A_{T 23} & A_{T 33}
\end{array}\right] \\
A_{T 11} & =\frac{1}{T_{0}^{2}} \int_{0}^{1} V^{\prime}\left(n_{e}+n_{i}\right) T^{2} d \rho \\
A_{T 12} & =\frac{1}{T_{0}} \int_{0}^{1} V^{\prime} \rho^{2}\left(n_{e}+n_{i}\right) T^{2} d \rho \\
A_{T 13} & =\frac{1}{T_{0}} \int_{0}^{1} V^{\prime} \rho^{4}\left(n_{e}+n_{i}\right) T^{2} d \rho \\
A_{T 22} & =\int_{0}^{1} V^{\prime} \rho^{4}\left(n_{e}+n_{i}\right) T^{2} d \rho \\
A_{T 23} & =\int_{0}^{1} V^{\prime} \rho^{6}\left(n_{e}+n_{i}\right) T^{2} d \rho \\
A_{T 11} & =\int_{0}^{1} V^{\prime} \rho^{8}\left(n_{e}+n_{i}\right) T^{2} d \rho
\end{aligned}
$$

The flux equation is represented by the matrix $\overleftrightarrow{\mathbf{A}}_{\psi}$ whose elements are given by 


$$
\begin{aligned}
\overleftrightarrow{\mathbf{A}}_{\psi} \equiv\left[\begin{array}{ccc}
A_{\psi 11} & A_{\psi 12} & A_{\psi 13} \\
A_{\psi 12} & A_{\psi 22} & A_{\psi 23} \\
A_{\psi 13} & A_{\psi 23} & A_{\psi 33}
\end{array}\right] \\
A_{\psi 11}=\frac{1}{\psi_{0}^{2}} \int_{0}^{1} V^{\prime} \frac{\left\langle 1 / R^{2}\right\rangle}{F(\rho) \eta_{\|}(\rho)} \psi^{2} d \rho \\
A_{\psi 12}=\frac{1}{\psi_{0}} \int_{0}^{1} V^{\prime} \rho^{2} \frac{\left\langle 1 / R^{2}\right\rangle}{F(\rho) \eta_{\|}(\rho)} \psi^{2} d \rho \\
A_{\psi 13}=\frac{1}{\psi_{0}} \int_{0}^{1} V^{\prime} \rho^{4} \frac{\left\langle 1 / R^{2}\right\rangle}{F(\rho) \eta_{\|}(\rho)} \psi^{2} d \rho \\
A_{\psi 22}=\int_{0}^{1} V^{\prime} \rho^{4} \frac{\left\langle 1 / R^{2}\right\rangle}{F(\rho) \eta_{\|}(\rho)} \psi^{2} d \rho \\
A_{\psi 23}=\int_{0}^{1} V^{\prime} \rho^{6} \frac{\left\langle 1 / R^{2}\right\rangle}{F(\rho) \eta_{\|}(\rho)} \psi^{2} d \rho \\
A_{\psi 11}=\int_{0}^{1} V^{\prime} \rho^{8} \frac{\left\langle 1 / R^{2}\right\rangle}{F(\rho) \eta_{\|}(\rho)} \psi^{2} d \rho
\end{aligned}
$$

The elements of the vector $\vec{R}$ contain the various sources and sinks, as well as the conductive and convective transport characteristics of the problem, and may be written as

$$
\begin{aligned}
R_{1} & =\frac{1}{n_{d 0}} \int_{0}^{1} V^{\prime}\left[-D_{d}\left(\frac{\partial n_{d}}{\partial \rho}\right)^{2}+\Gamma_{d} \frac{\partial n_{d}}{\partial \rho}+\left\langle S_{d}\right\rangle n_{d}\right] d \rho \\
& -\left.V^{\prime} D_{d} \frac{\partial n_{d}}{\partial \rho} \frac{n_{d}}{n_{d 0}}\right|_{\rho=1}+\left.V^{\prime} v_{d} \frac{n_{d}^{3}}{n_{d 0}}\right|_{\rho=1} \\
R_{2} & =\int_{0}^{1} V^{\prime}\left[-D_{d} \frac{\partial n_{d}}{\partial \rho} \frac{\partial}{\partial \rho}\left(\rho^{2} n_{d}\right)+\Gamma_{d} \frac{\partial}{\partial \rho}\left(\rho^{2} n_{d}\right)\right] d \rho \\
& +\int_{0}^{1} V^{\prime}\left\langle S_{d}\right\rangle n_{d} \rho^{2} d \rho-\left.V^{\prime} D_{d} \frac{\partial n_{d}}{\partial \rho} \rho^{2} n_{d}\right|_{\rho=1}+\left.V^{\prime} v_{d} \rho^{2} n_{d}^{3}\right|_{\rho=1}
\end{aligned}
$$




$$
\begin{aligned}
R_{3} & =\int_{0}^{1} V^{\prime}\left[-D_{d} \frac{\partial n_{d}}{\partial \rho} \frac{\partial}{\partial \rho}\left(\rho^{4} n_{d}\right)+\Gamma_{d} \frac{\partial}{\partial \rho}\left(\rho^{4} n_{d}\right)\right] d \rho \\
& +\int_{0}^{1} V^{\prime}\left\langle S_{d}\right\rangle n_{d} \rho^{4} d \rho-\left.V^{\prime} D_{d} \frac{\partial n_{d}}{\partial \rho} \rho^{4} n_{d}\right|_{\rho=1}+\left.V^{\prime} v_{d} \rho^{4} n_{d}^{3}\right|_{\rho=1}
\end{aligned}
$$

$$
\begin{aligned}
R_{4} & =\frac{1}{n_{t 0}} \int_{0}^{1} V^{\prime}\left[-D_{t}\left(\frac{\partial n_{t}}{\partial \rho}\right)^{2}+\Gamma_{t} \frac{\partial n_{t}}{\partial \rho}+\left\langle S_{t}\right\rangle n_{t}\right] d \rho \\
& -\left.V^{\prime} D_{t} \frac{\partial n_{t}}{\partial \rho} \frac{n_{t}}{n_{t 0}}\right|_{\rho=1}+\left.V^{\prime} v_{t} \frac{n_{t}^{3}}{n_{t 0}}\right|_{\rho=1}
\end{aligned}
$$

$$
\begin{aligned}
R_{5} & =\int_{0}^{1} V^{\prime}\left[-D_{t} \frac{\partial n_{t}}{\partial \rho} \frac{\partial}{\partial \rho}\left(\rho^{2} n_{t}\right)+\Gamma_{t} \frac{\partial}{\partial \rho}\left(\rho^{2} n_{t}\right)\right] d \rho \\
& +\int_{0}^{1} V^{\prime}\left\langle S_{t}\right\rangle n_{t} \rho^{2} d \rho-\left.V^{\prime} D_{t} \frac{\partial n_{t}}{\partial \rho} \rho^{2} n_{t}\right|_{\rho=1}+\left.V^{\prime} v_{t} \rho^{2} n_{t}^{3}\right|_{\rho=1}
\end{aligned}
$$

$$
\begin{aligned}
R_{6} & =\int_{0}^{1} V^{\prime}\left[-D_{t} \frac{\partial n_{t}}{\partial \rho} \frac{\partial}{\partial \rho}\left(\rho^{4} n_{t}\right)+\Gamma_{t} \frac{\partial}{\partial \rho}\left(\rho^{4} n_{t}\right)\right] d \rho \\
& +\int_{0}^{1} V^{\prime}\left\langle S_{t}\right\rangle n_{t} \rho^{4} d \rho-\left.V^{\prime} D_{t} \frac{\partial n_{t}}{\partial \rho} \rho^{4} n_{t}\right|_{\rho=1}+\left.V^{\prime} v_{t} \rho^{4} n_{t}^{3}\right|_{\rho=1}
\end{aligned}
$$

$$
\begin{aligned}
R_{7} & =\frac{1}{n_{\alpha 0}} \int_{0}^{1} V^{\prime}\left[-D_{\alpha}\left(\frac{\partial n_{\alpha}}{\partial \rho}\right)^{2}+\Gamma_{\alpha} \frac{\partial n_{\alpha}}{\partial \rho}+\left\langle S_{\alpha}\right\rangle n_{\alpha}\right] d \rho \\
& -\left.V^{\prime} D_{\alpha} \frac{\partial n_{\alpha}}{\partial \rho} \frac{n_{\alpha}}{n_{\alpha 0}}\right|_{\rho=1}+\left.V^{\prime} v_{\alpha} \frac{n_{\alpha}^{3}}{n_{\alpha 0}}\right|_{\rho=1}
\end{aligned}
$$$$
\begin{aligned}
R_{8} & =\int_{0}^{1} V^{\prime}\left[-D_{\alpha} \frac{\partial n_{\alpha}}{\partial \rho} \frac{\partial}{\partial \rho}\left(\rho^{2} n_{\alpha}\right)+\Gamma_{\alpha} \frac{\partial}{\partial \rho}\left(\rho^{2} n_{\alpha}\right)\right] d \rho \\
& +\int_{0}^{1} V^{\prime}\left\langle S_{\alpha}\right\rangle n_{\alpha} \rho^{2} d \rho-\left.V^{\prime} D_{\alpha} \frac{\partial n_{\alpha}}{\partial \rho} \rho^{2} n_{\alpha}\right|_{\rho=1}+\left.V^{\prime} v_{\alpha} \rho^{2} n_{\alpha}^{3}\right|_{\rho=1}
\end{aligned}
$$

$$
\begin{aligned}
R_{9} & =\int_{0}^{1} V^{\prime}\left[-D_{\alpha} \frac{\partial n_{\alpha}}{\partial \rho} \frac{\partial}{\partial \rho}\left(\rho^{4} n_{\alpha}\right)+\Gamma_{\alpha} \frac{\partial}{\partial \rho}\left(\rho^{4} n_{\alpha}\right)\right] d \rho \\
& +\int_{0}^{1} V^{\prime}\left\langle S_{\alpha}\right\rangle n_{\alpha} \rho^{4} d \rho-\left.V^{\prime} D_{\alpha} \frac{\partial n_{\alpha}}{\partial \rho} \rho^{2} n_{\alpha}\right|_{\rho=1}+\left.V^{\prime} v_{\alpha} \rho^{4} n_{\alpha}^{3}\right|_{\rho=1}
\end{aligned}
$$




$$
\begin{aligned}
R_{10} & =\frac{1}{n_{i m p}} \int_{0}^{1} V^{\prime}\left[-D_{i m p}\left(\frac{\partial n_{i m p}}{\partial \rho}\right)^{2}+\Gamma_{i m p} \frac{\partial n_{i m p}}{\partial \rho}\right] d \rho \\
& +\int_{0}^{1} V^{\prime}\left\langle S_{i m p}\right\rangle n_{i m p} d \rho \\
& -\left.V^{\prime} D_{i m p} \frac{\partial n_{i m p}}{\partial \rho} \frac{n_{i m p}}{n_{i m p}}\right|_{\rho=1}+\left.V^{\prime} v_{i m p} \frac{n_{i m p}^{3}}{n_{i m p 0}}\right|_{\rho=1} \\
R_{11} & =\int_{0}^{1} V^{\prime}\left[-D_{i m p} \frac{\partial n_{i m p}}{\partial \rho} \frac{\partial}{\partial \rho}\left(\rho^{2} n_{i m p}\right)+\Gamma_{i m p} \frac{\partial}{\partial \rho}\left(\rho^{2} n_{i m p}\right)\right] d \rho \\
& +\int_{0}^{1} V^{\prime}\left[\left\langle S_{i m p}\right\rangle n_{i m p} \rho^{2}\right] d \rho \\
& -\left.V^{\prime} D_{i m p} \frac{\partial n_{i m p}}{\partial \rho} \rho^{2} n_{i m p}\right|_{\rho=1}+\left.V^{\prime} v_{i m p} \rho^{2} n_{i m p}^{3}\right|_{\rho=1}
\end{aligned}
$$

$$
\begin{aligned}
R_{12} & =\int_{0}^{1} V^{\prime}\left[-D_{i m p} \frac{\partial n_{i m p}}{\partial \rho} \frac{\partial}{\partial \rho}\left(\rho^{4} n_{i m p}\right)+\Gamma_{i m p} \frac{\partial}{\partial \rho}\left(\rho^{4} n_{i m p}\right)\right] d \rho \\
& +\int_{0}^{1} V^{\prime}\left[\left\langle S_{i m p}\right\rangle n_{i m p} \rho^{4}\right] d \rho \\
& -\left.V^{\prime} D_{i m p} \frac{\partial n_{i m p}}{\partial \rho} \rho^{4} n_{i m p}\right|_{\rho=1}+\left.V^{\prime} v_{i m p} \rho^{4} n_{i m p}^{3}\right|_{\rho=1}
\end{aligned}
$$

$$
R_{13}=\frac{1}{T_{0}} \int_{0}^{1} V^{\prime}\left[-\left(n_{e} \chi_{e}+n_{i} \chi_{i}\right)\left(\frac{\partial T}{\partial \rho}\right)^{2}+\Gamma_{T} \frac{\partial T}{\partial \rho}\right] d \rho
$$$$
+\int_{0}^{1} V^{\prime}\left(\left\langle S_{E e}\right\rangle+\left\langle S_{E i}\right\rangle\right) T d \rho
$$$$
-\left.V^{\prime}\left(n_{e} \chi_{e}+n_{i} \chi_{i}\right) \frac{\partial T}{\partial \rho} \frac{T}{T_{0}}\right|_{\rho=1}+\left.\frac{5}{2} V^{\prime} \Gamma_{T} \frac{T^{2}}{T_{0}}\right|_{\rho=1}
$$

$$
\begin{aligned}
R_{14} & =\int_{0}^{1} V^{\prime}\left[-\left(n_{e} \chi_{e}+n_{i} \chi_{i}\right) \frac{\partial T}{\partial \rho} \frac{\partial}{\partial \rho}\left(\rho^{2} T\right)+\Gamma_{T} \frac{\partial T}{\partial \rho}\right] d \rho \\
& +\int_{0}^{1} V^{\prime}\left(\left\langle S_{E e}\right\rangle+\left\langle S_{E i}\right\rangle\right) \rho^{2} T d \rho \\
& -\left.V^{\prime}\left(n_{e} \chi_{e}+n_{i} \chi_{i}\right) \frac{\partial T}{\partial \rho} \rho^{2} T\right|_{\rho=1}+\left.\frac{5}{2} V^{\prime} \Gamma_{T} \rho^{2} T^{2}\right|_{\rho=1}
\end{aligned}
$$




$$
\begin{aligned}
R_{15} & =\int_{0}^{1} V^{\prime}\left[-\left(n_{e} \chi_{e}+n_{i} \chi_{i}\right) \frac{\partial T}{\partial \rho} \frac{\partial}{\partial \rho}\left(\rho^{4} T\right)+\Gamma_{T} \frac{\partial T}{\partial \rho}\right] d \rho \\
& +\int_{0}^{1} V^{\prime}\left(\left\langle S_{E e}\right\rangle+\left\langle S_{E i}\right\rangle\right) \rho^{4} T d \rho \\
& -\left.V^{\prime}\left(n_{e} \chi_{e}+n_{i} \chi_{i}\right) \frac{\partial T}{\partial \rho} \rho^{4} T\right|_{\rho=1}+\left.\frac{5}{2} V^{\prime} \Gamma_{T} \rho^{4} T^{2}\right|_{\rho=1} \\
R_{16} & =\frac{1}{\psi_{0}} \int_{0}^{1} V^{\prime}\left[-\frac{1}{F(\rho)}\left\langle\frac{|\nabla \rho|^{2}}{R^{2}}\right\rangle\left(\frac{\partial \psi}{\partial \rho}\right)^{2}+\left\langle R_{\psi}\right\rangle \psi\right] d \rho \\
R_{17} & =\int_{0}^{1} V^{\prime}\left[-\frac{1}{F(\rho)}\left\langle\frac{|\nabla \rho|^{2}}{R^{2}}\right\rangle \frac{\partial \psi}{\partial \rho} \frac{\partial}{\partial \rho}\left(\rho^{2} \psi\right)+\left\langle R_{\psi}\right\rangle \rho^{2} \psi\right] d \rho \\
R_{18} & =\int_{0}^{1} V^{\prime}\left[-\frac{1}{F(\rho)}\left\langle\frac{|\nabla \rho|^{2}}{R^{2}}\right\rangle \frac{\partial \psi}{\partial \rho} \frac{\partial}{\partial \rho}\left(\rho^{4} \psi\right)+\left\langle R_{\psi}\right\rangle \rho^{4} \psi\right] d \rho
\end{aligned}
$$

where $R_{\psi}$ in Eqs. $(6.105-6.107)$ is given by

$$
R_{\psi} \equiv \frac{\left\langle\vec{E}_{e x t} \cdot \vec{B}\right\rangle}{\left\langle 1 / R^{2}\right\rangle F(\rho)}
$$

In summary, by finding the Lagrangian $\mathcal{L}$, by assuming forms for the trial functions, and by taking the variations of $\mathcal{L}$ with respect to all the variational parameters appearing in the trial functions, it has been possible to reduce the system of partial differential equations to a system of coupled ordinary differential equations.

\subsection{Sawtooth Model}

Tokamaks are subject to various global, and local instabilities which can not be predicted by the transport equations presented here. Most of the macroscopic instabilities observed in tokamaks can be attributed to certain MHD modes. 
Sawtooth oscillations were first observed in the ST tokamak. [62] There it was found, by observing the soft X-rays emitted from the central part of the plasma column, that the emission undergoes oscillations having a sawtooth time dependence. This change in the X-ray emission is primarily due to a change in the electron temperature.

Various models have been developed which attempt to explain the sawtooth oscillations. In particular, Kodomtsev [63] proposed an explanation as a "reconnection model", in which the $\mathrm{m}=1$ magnetic island grows by reconnecting the magnetic flux until it encompasses all the plasma inside the $q=1$ region. Subsequently, the hot plasma is expelled from the region $q<1$ resulting in the flattening of the density, temperature, and current density profiles.

In general, sawtooth oscillations result from a competition between two effects: the peaking of the temperature and thus the current density profile, and by the triggering of an $\mathrm{m}=1, \mathrm{n}=1$ MHD like mode once the $q$ value at the plasma center, $q_{0}$, falls below unity.[64]

For the sawtooth model developed here it is necessary to address the following questions.

1. What are the necessary conditions for the occurrence of sawteeth?

2. What is the sawtooth collapse time?

3. What is the heating rate of the sawtooth?

4. What is the sawtooth repetition time?

5. What are the profile changes due to the sawtooth collapse?

It has been shown that the condition for sawtooth activity is $q_{0} \approx 1$.[64] In estimating the sawtooth collapse time there is no agreement between theory or experiment. For example, in the JET tokamak the experimentally measured collapse time is $\tau_{c} \sim 100 \mu \mathrm{sec}$ [65] while the predicted collapse 
time from Kodomtsev's [63] reconnection model is $\sim 5$ msec. In this model the collapse time is chosen to be $100 \mu s e c$. By analyzing the one dimensional electron heat equation, it has shown that the temperature rise of the sawtooth "appears to be predominantly due to resistive heating".[64]

The sawtooth period is a strong function of the plasma parameters and the operating conditions. A good estimate of the sawtooth period, $\tau_{\boldsymbol{s}}$, is given by[66]

$$
\tau_{s} \approx\left(\frac{r_{i}}{a}\right)^{6 / 5}\left(\frac{T_{i}}{T_{e}}\right)^{1 / 5} \tau_{A}^{2 / 5} \tau_{h}^{2 / 5} \omega_{*}^{2 / 5} \tau_{R}^{3 / 5}
$$

where $r_{i}$ is the radius at which $q=1, a$ is the plasma minor radius, $\tau_{A}$ represents the Alfven transit time across the plasma radius

$$
\tau_{A}=\frac{m \rho^{1 / 2}}{k B_{z}}
$$

where $B_{z}$ is the toroidal magnetic field, $m$, and $k$ are respectively the poloidal and toroidal mode numbers, and $\rho$ is the mass density. The heating rate $1 / \tau_{h}$ at the magnetic axis may be calculated from

$$
\tau_{h} \approx \frac{3}{2} n_{e 0} \frac{T_{e 0}}{\eta_{0} J_{\phi 0}^{2}}
$$

where $\eta_{0}$, and $J_{\phi 0}$ are respectively the resistivity and the toroidal current density at the plasma center. $\tau_{R}$ is the resistive skin time across the plasma radius and is given by

$$
\tau_{R}=\frac{a^{2}}{\eta}
$$

The parameter $\omega_{*}$ in Eq. (6.110) represents the plasma diamagnetic frequency and has the form

$$
\omega_{*}=\frac{m}{e B_{z} r_{i}} \frac{d T}{d r}\left(r_{i}\right)
$$


The prediction of $\tau_{s}$ from Eq. (6.110) does not apply in general. For example, in neutral beam and RF heated plasmas it has been observed that the sawtooth period becomes very long $>2 s e c$. These types of sawtooth oscillations are called "monster sawteeth" and have been observed in both the JET and TFTR tokamaks $[67,68,69]$ The effects of these types of sawtooth oscillations on plasma transport can be simulated by arbitrarily choosing the sawtooth period.

After the sawtooth collapse the temperature and density profiles are changed. In general, the profiles are flattened up to the inversion radius, $\rho_{i}$ which corresponds to $q=1$ i.e. $\rho_{i}=\rho(q=1)$. Another interesting point is the mixing radius, $\rho_{m}$, beyond which the profiles remain unchanged. These qualitative arguments regarding the profile changes are drawn upon examination of experimental data. The name inversion radius is derived from the observation that during sawtooth activity the X-ray emission observed at outer radii indicates that the sawtooth is inverted.

During the sawtooth collapse the temperature, particle density and current density profiles are changed but the total plasma density and the total plasma current remain constant. From experimental data in DIII-D and other tokamaks, scalings for the location of the mixing radius $\rho_{m}$ have been determined. In particular, Snider [70] has reported the following scaling for $\rho_{m}$

$$
\rho_{m}=\frac{1}{q_{95}}+0.27
$$

where $q_{95}$ is the safety factor at the $95 \%$ flux surface.

It is important to determine the shape of the profile after the collapse since it is required as a new initial condition for evolving the plasma parameters. For the variational transport model developed in this thesis it is important to estimate the value of the variational parameters after the collapse since they determine the actual shape of the profiles. For the trial function used there are three variational parameters and thus values for these three parameters must be obtained after the sawtooth collapse. 
The temperature profile is changing during a sawtooth collapse. Due to the fact that the collapse is very fast no energy is transported across the plasma via the standard convective and conductive processes during the time frame of the collapse. However, due to the temperature change inside the mixing radius the radiation balance changes. For example the Bremmstrahlung radiation level changes during the sawtooth collapse.[71] If the profile shape for the density and the temperature is known before and after the collapse the difference in the Bremmstrahlung radiated power can be estimated. If $E_{1}$ is the total energy content of the plasma prior to the collapse and $E_{2}$ is the total energy after the collapse then

$$
E_{2}=E_{1}-E_{B}
$$

where $E_{B}$ is the energy lost from the plasma due to Bremmstrahlung radiation. By writing

$$
E_{2}=\epsilon E_{1}
$$

where $\epsilon=1-E_{B} / E_{1}$, the energy conservation condition given by Eq. (6.117) can be used as one of the conditions needed for estimating the change of the temperature profile. For arguments similar to those developed for the estimation of the density profile changes, the temperature profile remains unchanged at radii greater than the mixing radius $\rho_{m}$ i.e. for $\rho_{m}<\rho<1$ the temperature profile remains unchanged. The two conditions outlined above may be used to determine two of the three parameters appearing in the temperature trial function Eq. (6.34). Therefore for a complete solution another condition is required. This condition can be deduced from the observation that the temperature profile inside the inversion radius $\rho_{i}$ is flattened.[71] Thus if the location of the inversion radius is known, the peak temperature after the collapse is equal to the temperature at $\rho=\rho_{i}$ before the collapse. It is then possible to estimate the central temperature drop $\Delta T$ due to the sawtooth collapse.In particular $\Delta T$ may be written as

$$
\Delta T=T_{b}(\rho=0)-T_{b}\left(\rho=\rho_{i}\right)
$$


The inversion radius can be obtained by either using the scaling relation[70]

$$
\rho_{i} \simeq \frac{1}{q_{95}}
$$

or by finding the radius below which the safety factor becomes less than one.

In summary then the determination of the temperature profile after the sawtooth collapse is found by using the equations.

$$
\begin{array}{ll}
\int_{V} E_{2} d V=\int_{V} \epsilon E_{1} d V & \text { Energy conservation } \\
\Delta T=T_{b}(\rho=0)-T_{b}\left(\rho=\rho_{i}\right) & \text { Given Temperature drop }
\end{array}
$$

and by minimizing the integral

$$
I=\int_{\rho_{m}}^{1}\left(T_{b}-T_{a}\right)^{2} d \rho
$$

Next in order to determine the change in the flux profile $\psi$ the following assumptions are made.

1. The total plasma current is conserved during the sawtooth collapse.

2. The axial current density $J(\rho=0)$ changes by a prescribed amount during the sawtooth collapse. Soltwisch [72] has estimated that the $J(\rho=0)$ changes by approximately $8 \%$ during the collapse.

3. The flux profile does not change for radii greater than the mixing radius $\rho_{m}$

Mathematically the above conditions are expressed as follows.

$$
\begin{aligned}
\left.I_{p}(\rho)\right|_{\rho=\rho_{m}}= & -\left.\frac{1}{\mu_{0}} V^{\prime} \frac{\partial \psi}{\partial \rho}\left\langle\frac{|\nabla \rho|^{2}}{R^{2}}\right\rangle\right|_{\rho=\rho_{m}}=\text { Constant } \\
\Delta J(\rho=0) \simeq & 8 \% \\
\text { Minimize } & \int_{\rho_{m}}^{1}\left(\psi_{b}-\psi_{a}\right)^{2} d \rho
\end{aligned}
$$


The mathematical model used for determining the shape of the density profile after the sawtooth collapse is based on the following: First, the total number of particles is conserved during the collapse, second, the value of the density at the mixing radius $\rho_{m}$ before the collapse equals to the density after the collapse, third, the shape of the profile remains unchanged for $\rho_{m}<\rho<1$. Furthermore, the value of the density at the plasma center, $\rho=0$ equals to the density at the inversion radius $\rho_{i}$.

Mathematically, if $n_{b}$ corresponds to the profile before the collapse and $n_{a}$ corresponds to the profile after the collapse, the above ideas are expressed as follows

$$
\begin{array}{ll}
\int_{V} n_{b} d V=\int_{V} n_{a} d V & \text { Particle conservation } \\
n_{b}\left(\rho_{m}\right)=n_{a}\left(\rho_{m}\right) & \text { Fixed density at } \rho_{m}
\end{array}
$$

and by minimizing the integral

$$
I=\int_{\rho_{m}}^{1}\left(n_{b}-n_{a}\right)^{2} d \rho
$$

the difference between the before and after profiles is minimized between $\rho_{m}$ and 1 .

\subsection{Relating $\tau_{E}$ to $\chi$}

The parameters that characterize the confinement of energy in a tokamak plasma are: the global energy confinement time $\tau_{E}$ for the volume averaged (0-D) model, and the thermal diffusivity $\chi$ in the one dimensional model. Since $\tau_{E}$ is a number and $\chi$ is a function, it is not possible to infer a unique $\chi$ once the global energy confinement time $\tau_{E}$ is known. In general however the global behavior represented by $\tau_{E}$ is a conglomeration of the local plasma behavior which may be represented by $\chi$, and there is a correspondence between the energy confinement time and the local plasma transport characteristics. This correspondence is valid only when volume averaged 
quantities are considered. In deriving the zero dimensional plasma transport equations in chapter 2 , the global energy confinement time $\tau_{E}$ was defined as

$$
\tau_{E}=\frac{E}{P}
$$

where $E$ is the energy content of the plasma

$$
E=\frac{3}{2} \sum_{j} \int_{V} n_{j} T_{j} d V
$$

and where $P$ represents the plasma heating power. In particular $P$ is given by

$$
P=P_{\text {oh }}+P_{\alpha}-P_{\text {rad }}
$$

The power lost due to conductive processes is equal to $P$ and is given by

$$
P=\int_{V} Q_{\text {loss }} d V
$$

the power density $Q$ has the form

$$
Q=\frac{1}{V^{\prime}} \frac{\partial}{\partial \rho} V^{\prime}\left(n_{e} \chi_{e}+n_{i} \chi_{i}\right)|\nabla \rho|^{2} \frac{\partial T}{\partial \rho}
$$

Therefore Eq. (6.127) can be written as

$$
\tau_{E}=-\frac{3}{2} \frac{\int_{0}^{1} V^{\prime}\left(n_{e}+n_{i}\right) T d \rho}{\left[V^{\prime}\left(n_{e} \chi_{e}+n_{i} \chi_{i}\right)|\nabla \rho|^{2} \frac{\partial T}{\partial \rho}\right]_{\rho=1}}
$$

It is not possible to obtain a unique relation between $\tau_{E}$ and $\chi$ unless the functional form of both $\chi_{e}$ and $\chi_{i}$ is specified. By assuming that the electron thermal diffusivity $\chi_{e}$ and the ion thermal diffusivity $\chi_{i}$ have the 
same functional dependance but are modulated by some constant factor $f_{\chi_{i}}$ then a simple model for $\chi_{e}$ and $\chi_{i}$ is

$$
\begin{aligned}
& \chi_{e}=C_{\chi} \exp \left[\nu_{\chi_{e}} \rho^{2}\right] \\
& \chi_{i}=f_{\chi_{i}} C_{\chi} \exp \left[\nu_{\chi_{i}} \rho^{2}\right]
\end{aligned}
$$

The parameters $\nu_{\chi_{e}}$ and $\nu_{\chi_{i}}$ determine the shape of the profiles and are assumed known. The parameter $C_{\chi}$ is given by

$$
C_{\chi}=-\frac{3}{2} \frac{\int_{0}^{1} V^{\prime}\left(n_{e}+n_{i}\right) T d \rho}{\tau_{E}\left[V^{\prime}\left(n_{e} \exp \left[\nu_{\chi_{e}}\right]+n_{i} f_{\chi_{i}} \exp \left[\nu_{\chi_{i}}\right]\right)|\nabla \rho|^{2} \frac{\partial T}{\partial \rho}\right]_{\rho=1}}
$$

By using Eq. (6.135) the parameter $C_{\chi}$ can be calculated as the plasma thermodynamic variables evolve and as the global energy confinement time changes.

\subsection{Solution Procedure}

As has been shown from the previous analysis, the problem of finding solutions to the plasma transport equations is reduced to the problem of solving the system of coupled ordinary differential equations given by Eq. (6.45). The variables to be evolved are the variational parameters.

In order to start the solution the initial conditions must be provided. In a standard PDE problem the initial conditions are given in the form of a radial profile. In this analysis the initial conditions are given by specifying values for the variational parameters, which actually dictate a shape for the initial profile. For example, by setting the shape parameters equal to zero (i.e. $\alpha_{j}=0, \beta_{j}=0$ ) the initial conditions are parabolic profiles. Besides the shape parameters, initial values must be provided for the central values of the thermodynamic variables $n_{j}, T$, and the flux $\psi$. 
For the solution to proceed, the boundary conditions at the plasma edge must be given. In general, the parameters $\gamma_{j}$ appearing in the trial functions, are determined as functions of the edge values which can be fixed or floating (functions of time) by using Eqs. $(6.27,6.28)$.

Once the initial and boundary conditions have been specified the next step is to determine the shape of the magnetic surfaces. This is required for the computation of the various flux surface averages. The determination of the magnetic surfaces is obtained by solving the Grad-Shafranov equation. The Grad-Shafranov equilibria are obtained by using the variational equilibrium model developed by Haney. [73] This variational Grad-Shafranov solver is characterized by fast execution times, simplicity, and robustness thereby making it ideal for calculating the flux surface shapes required for performing the various flux surface averages. In order to completely define this fixed boundary equilibrium problem the following parameters must be provided.

$\begin{array}{ll}R_{0} & \text { Plasma major radius } \\ a & \text { Plasma minor radius } \\ \kappa_{u}\left(\kappa_{l}\right) & \text { Plasma upper (lower) elongation } \\ \delta_{u}\left(\delta_{l}\right) & \text { Plasma upper (lower) triangularity } \\ B_{0} & \text { Toroidal magnetic field at } R_{0} \\ P & \text { Plasma pressure } \\ F & \text { Toroidal Flux Function }\end{array}$

The functions $P$, and $F$ are obtained from the transport equations and the rest of the variables listed above characterize the tokamak geometry under consideration. In particular the pressure $P$ is given by

$$
P=\left(n_{e}+n_{i}\right) T
$$

and the the function $F$ is given by Eq. (2.84) which is repeated here for convenience.

$$
\frac{\partial \psi}{\partial \rho} \frac{1}{V^{\prime}(\rho)} \frac{\partial}{\partial \rho}\left[V^{\prime}(\rho)\left\langle\frac{|\nabla \rho|^{2}}{R^{2}}\right\rangle \frac{\partial \psi}{\partial \rho}\right]=\mu_{0} P^{\prime}(\rho)+\left\langle\frac{1}{R^{2}}\right\rangle F F^{\prime}
$$




\subsection{Computer Implementation}

The theory developed in the previous sections has been implemented in a computer program called "MITra". This program, written in the MPPL programing language has been adapted to run on VAX stations and on the CRAY supercomputer. The system of ordinary differential equations is solved by calling the IMSL routine DVERK. This routine is based on a fifth and sixth order Runge-Kutta method. The code has been segmented and thus the user has the ability to easily modify the desired subroutine to perform the desired studies. For example the form of the diffusivity $\chi$ is given in a separate small unit whose manipulation is easy. Also the auxiliary power is given in a separate subroutine thereby enabling the user to change the auxiliary power deposition profiles with ease.

\subsection{Sample results of the $1 \frac{1}{2}-$ D MITra trans- port code}

The volume averaged $(0-\mathrm{D})$ transport model and the $1 \frac{1}{2}-\mathrm{D}$ transport model are fundamentally different in their representation of the plasma behavior. The 0-D model evolves the thermodynamic variables under the assumption of fixed profiles. However, even though the two models include different representation of the underlying physics, the results obtained under similar qualitative assumptions are similar. For example qualitative similar results are obtained when the temperature evolution is investigated. On Fig. 6.1 the 0-D temperature evolution for the CIT tokamak under Goldston H-mode scaling is compared to the temperature evolution obtained from the code MITra. The simulation is obtained for $\chi=C_{\chi} \exp \left[\rho^{2}\right]$ where $C_{\chi}$ is found from Eq. (6.135). Note that the final equilibrium temperature is similar. In particular the 0 -D model equilibrates at $T=33 \mathrm{keV}$, and the equilibrium obtained by MITra is $T=28 \mathrm{keV}$. This difference in the equilibration temperature can be decreased by changing the functional dependance of $\chi$. 

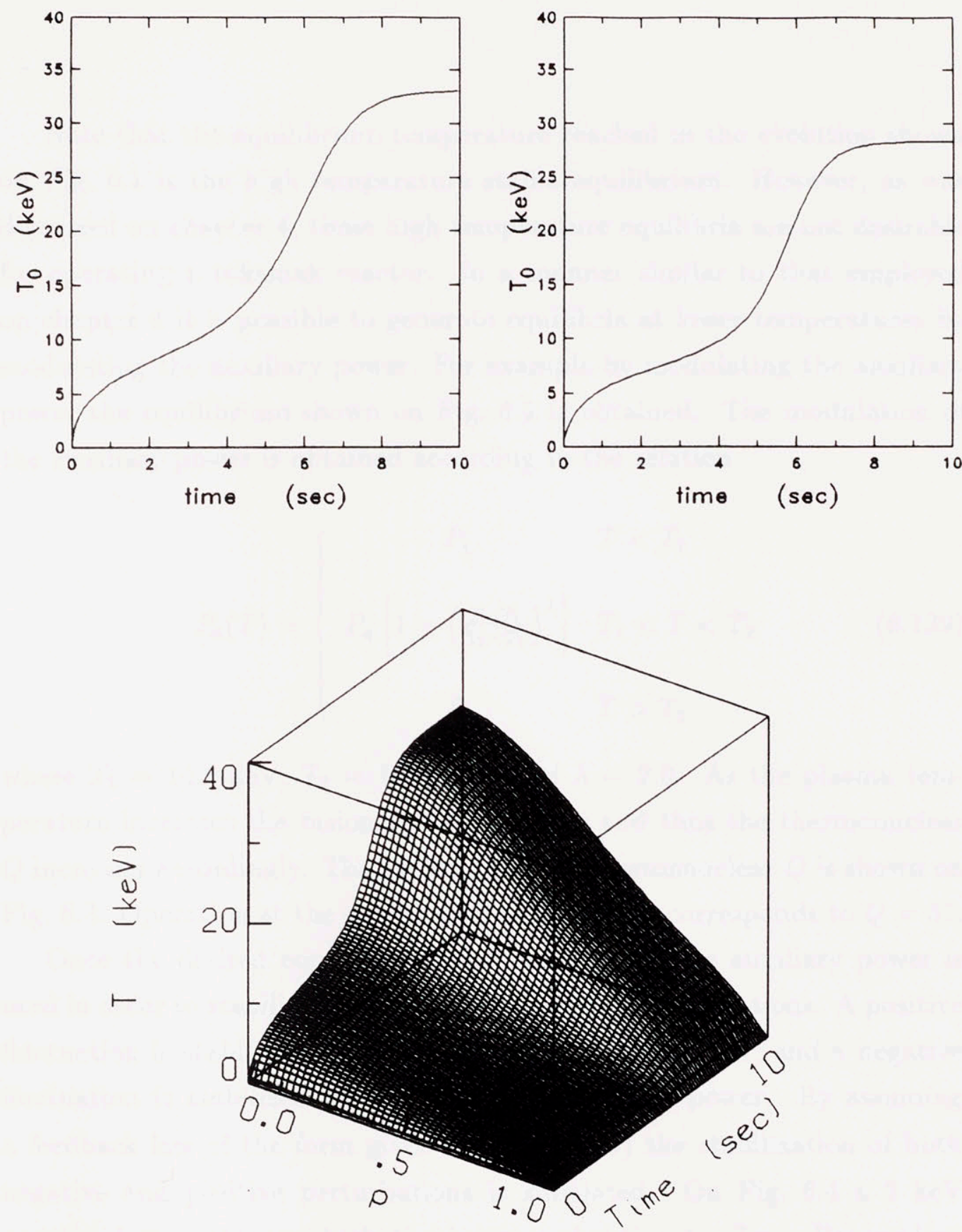

Figure 6.1: Clockwise from top left corner: 0-D temperature evolution of the peak temperature (assuming parabolic profiles) and Goldston H-mode scaling, the evolution of the peak temperature from the MITra code with $\chi=C_{\chi} \exp \left[\rho^{2}\right]$ where $C_{\chi}$ is determined from Eq. (6.135) for Goldston Hmode scaling. Also shown is the evolution of the temperature profile. 
Note that the equilibrium temperature reached in the evolution shown on Fig. 6.1 is the high temperature stable equilibrium. However, as was discussed on chapter 4 , these high temperature equilibria are not desirable for operating a tokamak reactor. In a manner similar to that employed on chapter 4 it is possible to generate equilibria at lower temperatures by modulating the auxiliary power. For example by modulating the auxiliary power the equilibrium shown on Fig. 6.2 is obtained. The modulation of the auxiliary power is obtained according to the relation

$$
P_{a}(T)=\left\{\begin{array}{cl}
P_{a} & T<T_{1} \\
P_{a}\left[1-\left(\frac{T-T_{1}}{T_{2}-T_{1}}\right)^{\lambda}\right] & T_{1}<T<T_{2} \\
0 & T>T_{2}
\end{array}\right.
$$

where $T_{1}=14.0 \mathrm{keV}, T_{2}=16.0 \mathrm{keV}$, and $\lambda=2.0$. As the plasma temperature increases the fusion power increases and thus the thermonuclear $Q$ increases accordingly. The evolution of the thermonuclear $Q$ is shown on Fig. 6.3. Operation at the equilibrium temperature corresponds to $Q=51$.

Once the desired equilibrium has been reached the auxiliary power is used in order to stabilize both positive and negative fluctuations. A positive fluctuation is stabilized by decreasing the auxiliary power, and a negative fluctuation is stabilized by increasing the auxiliary power. By assuming a feedback law of the form given by Eq. (6.139) the stabilization of both negative and positive perturbations is simulated. On Fig. 6.4 a $2 \mathrm{keV}$ negative temperature perturbation is imposed at time $t=7 \mathrm{sec}$. Responding to this perturbation is the auxiliary power which by increasing brings the system back to equilibrium.

Similarly the stabilization of a $1 \mathrm{keV}$ positive temperature fluctuation is simulated on Fig. 6.5. In this case by decreasing the auxiliary power the plasma looses energy resulting in a temperature decrease and thus stabilization of the perturbation. 

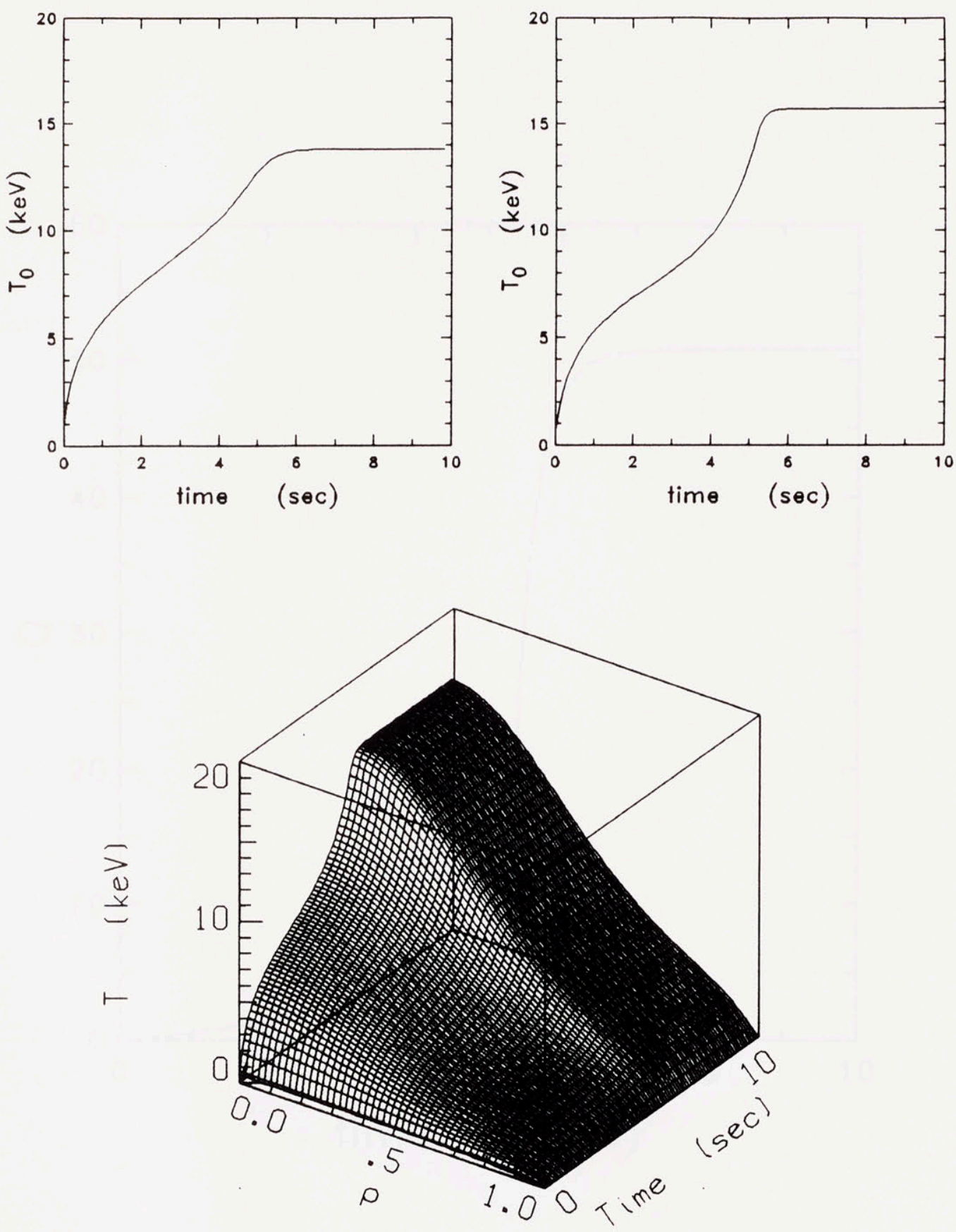

Figure 6.2: Clockwise from top left corner: 0-D temperature evolution of the peak temperature with auxiliary power modulation (Goldston H-mode scaling), the evolution of the peak temperature from the MITra code with centrally deposited and modulated auxiliary power and with diffusivity of the form $\chi=C_{\chi} \exp \left[\rho^{2}\right]$ where $C_{\chi}$ is determined from Eq. (6.135). Also shown is the evolution of the temperature profile. 


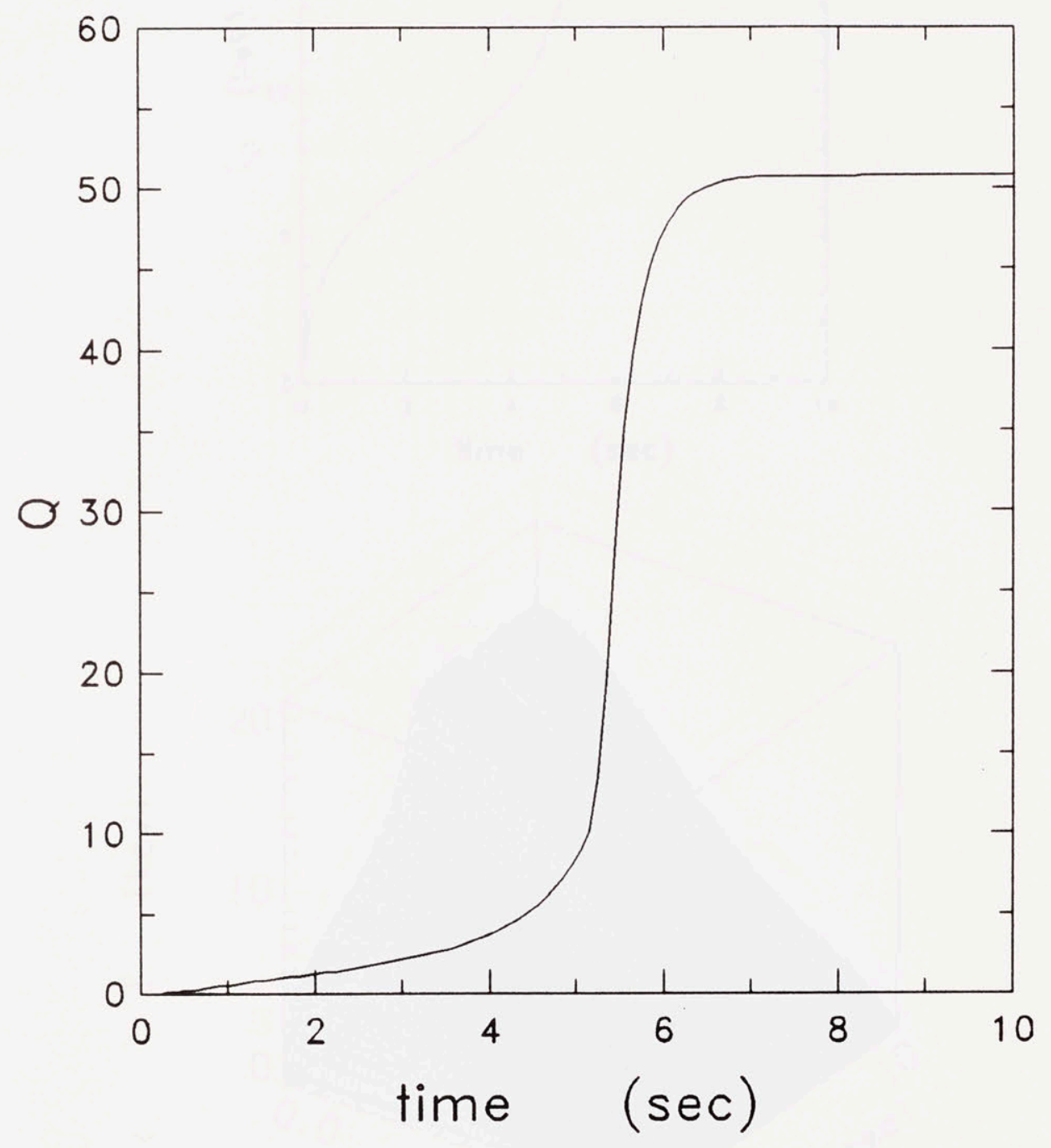

Figure 6.3: Evolution of the thermonuclear $Q$ corresponding to Fig. 6.2 for CIT under Goldston H-mode confinement and for $\chi=C_{\chi} \exp \left[\rho^{2}\right]$ where $C_{\chi}$ is found from Eq. (6.135) 

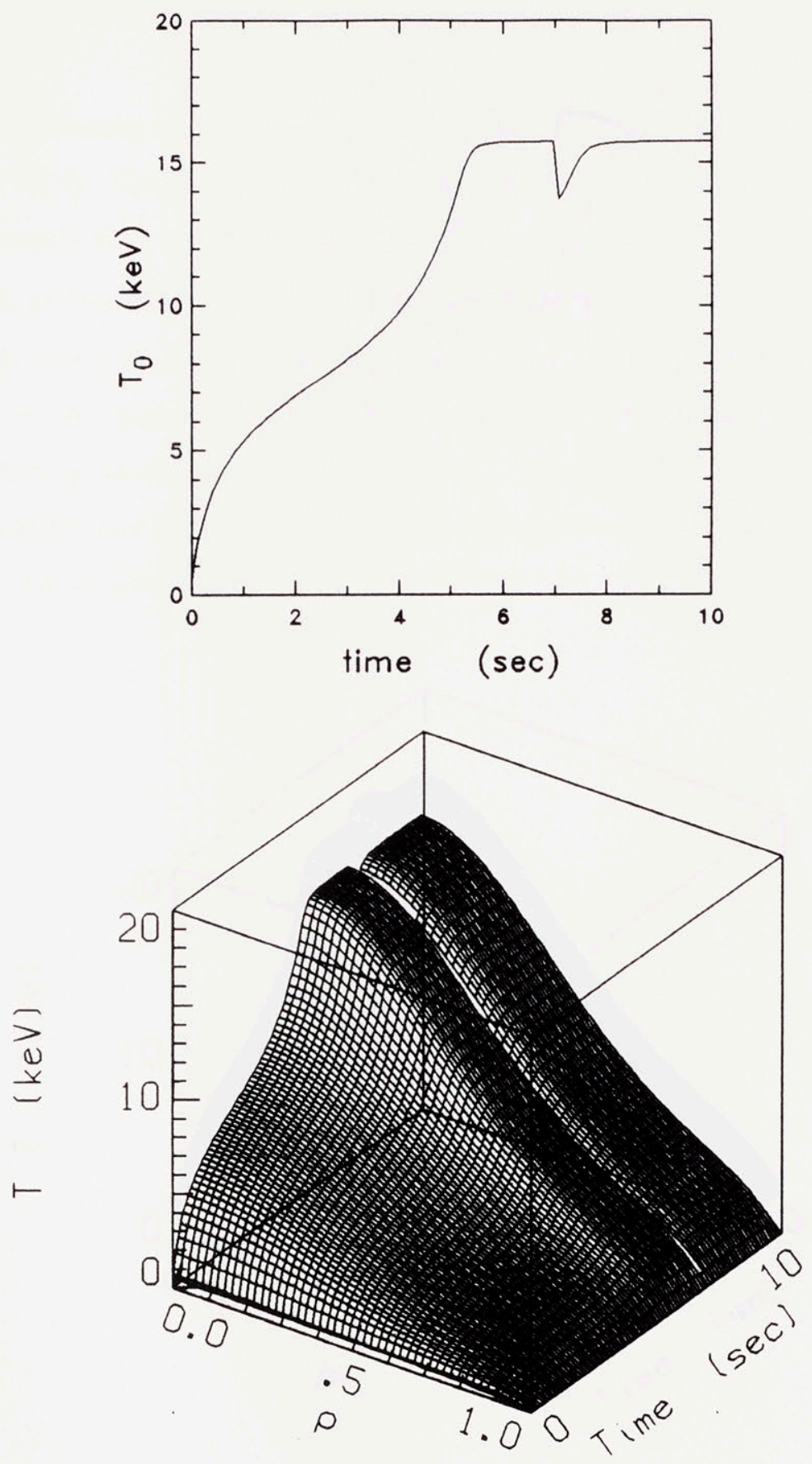

Figure 6.4: Top figure shows the trace of the peak temperature of a CIT discharge under Goldston H-mode scaling. Note that once the equilibrium is reached a $2 \mathrm{keV}$ negative perturbation is stabilized by auxiliary power modulation. The bottom plot shows the complete evolution of the temperature profiles. The form of the the $\chi$ is $\chi=C_{\chi} \exp \left[\rho^{2}\right]$ where $C_{\chi}$ is determined from Eq. (6.135). 


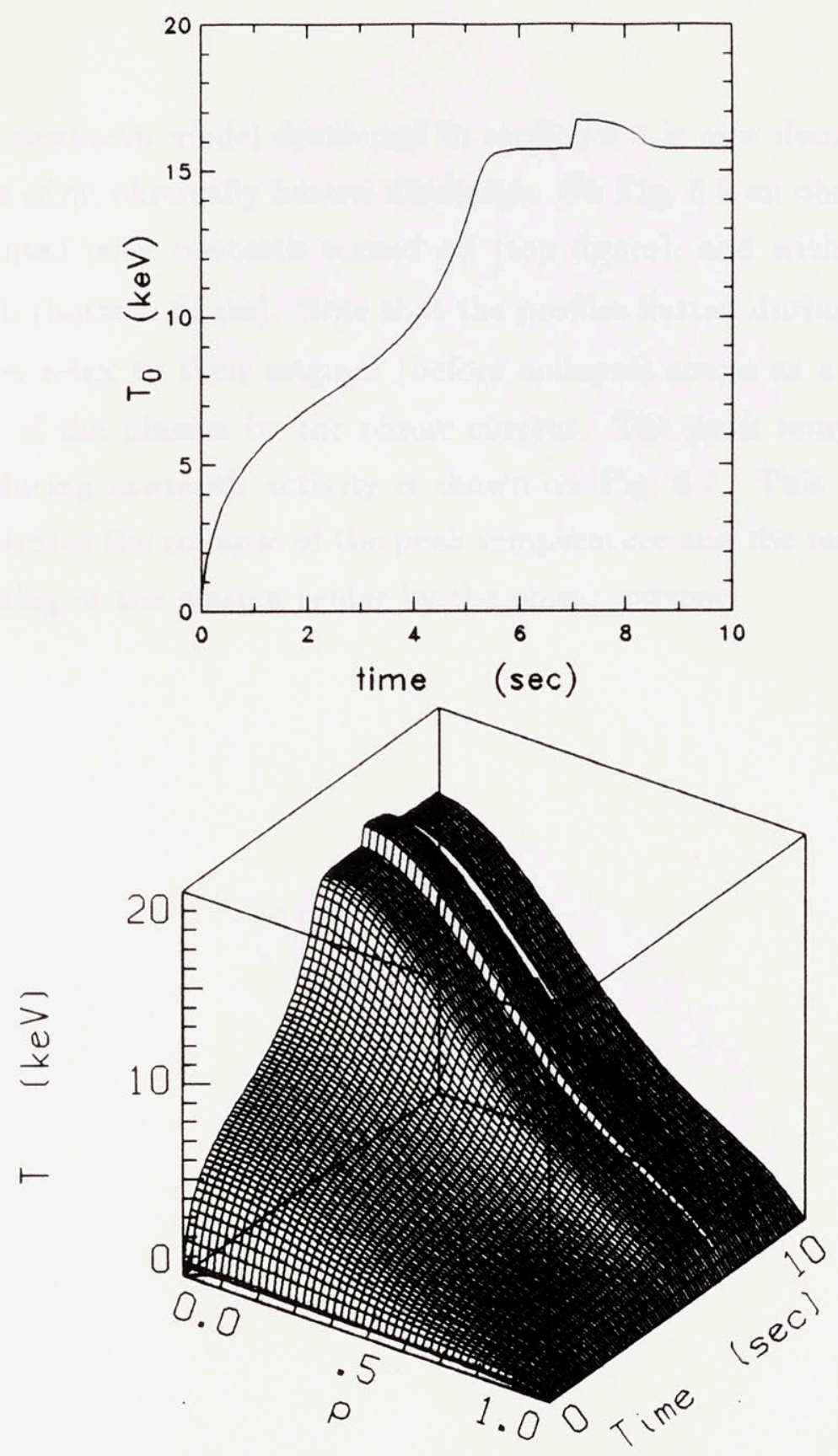

Figure 6.5: Top figure shows the trace of the peak temperature of a CIT discharge under Goldston $\mathrm{H}$-mode scaling. Note that once the equilibrium is reached a $1 \mathrm{keV}$ positive perturbation is stabilized by auxiliary power modulation. The bottom plot shows the complete evolution of the temperature profiles. The form of the the $\chi$ is $\chi=C_{\chi} \exp \left[\rho^{2}\right]$ where $C_{\chi}$ is determined from Eq. (6.135). 
The sawtooth model developed in section 6.3 is now demonstrated for the case of an ohmically heated discharge. On Fig. 6.6 an ohmic discharge is presented with sawteeth turned off (top figure), and with fixed period sawteeth (bottom figure). Note that the profiles flatten during the collapse and then relax to their original (before collapse) shape as a result of the heating of the plasma by the ohmic current. The peak temperature evolution during sawtooth activity is shown on Fig. 6.7. This figure clearly demonstrates the collapse of the peak temperature and the recovery due to the heating of the plasma center by the ohmic current. 

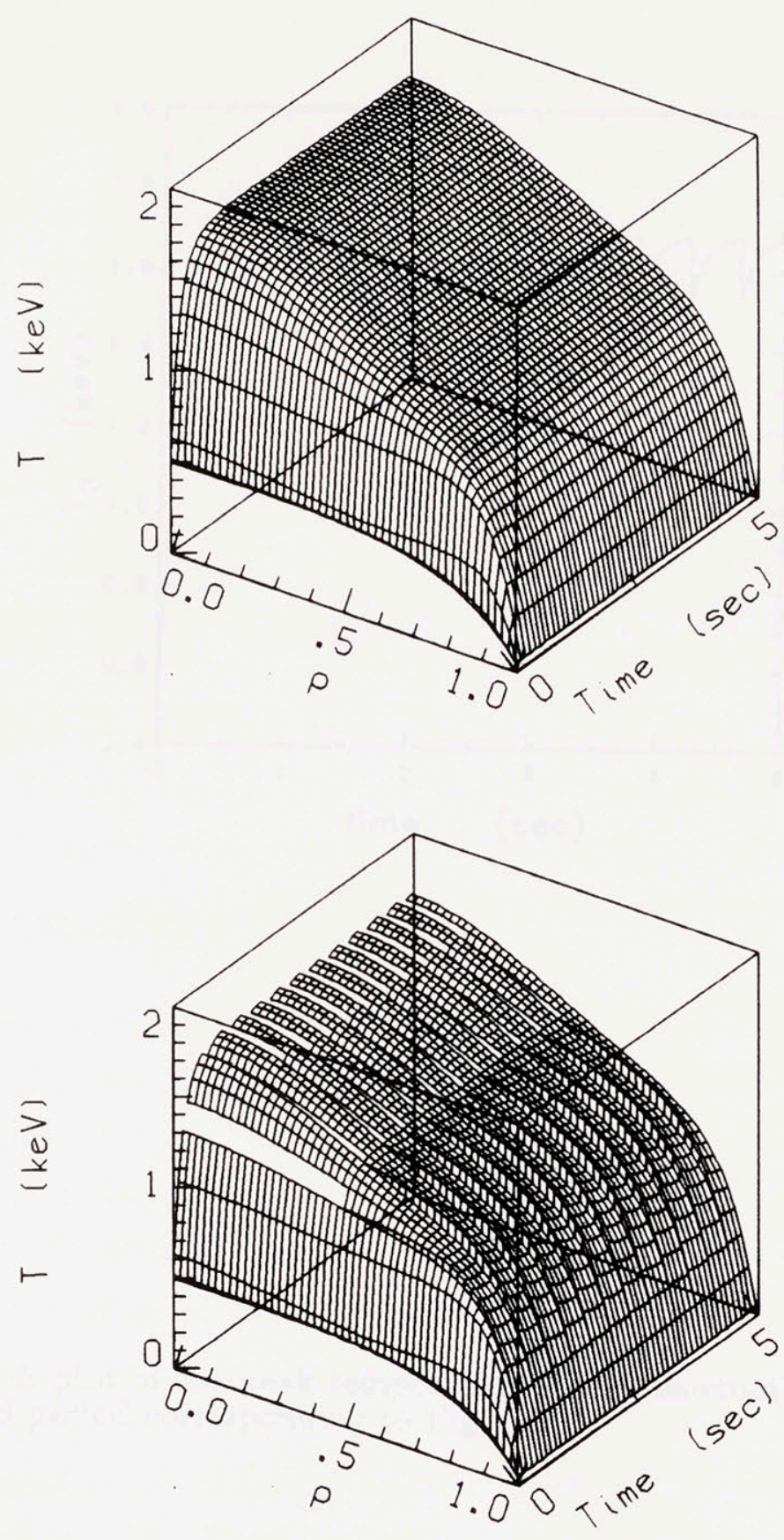

Figure 6.6: Ohmic evolution without sawteeth (top figure) and with sawteeth (bottom figure). 


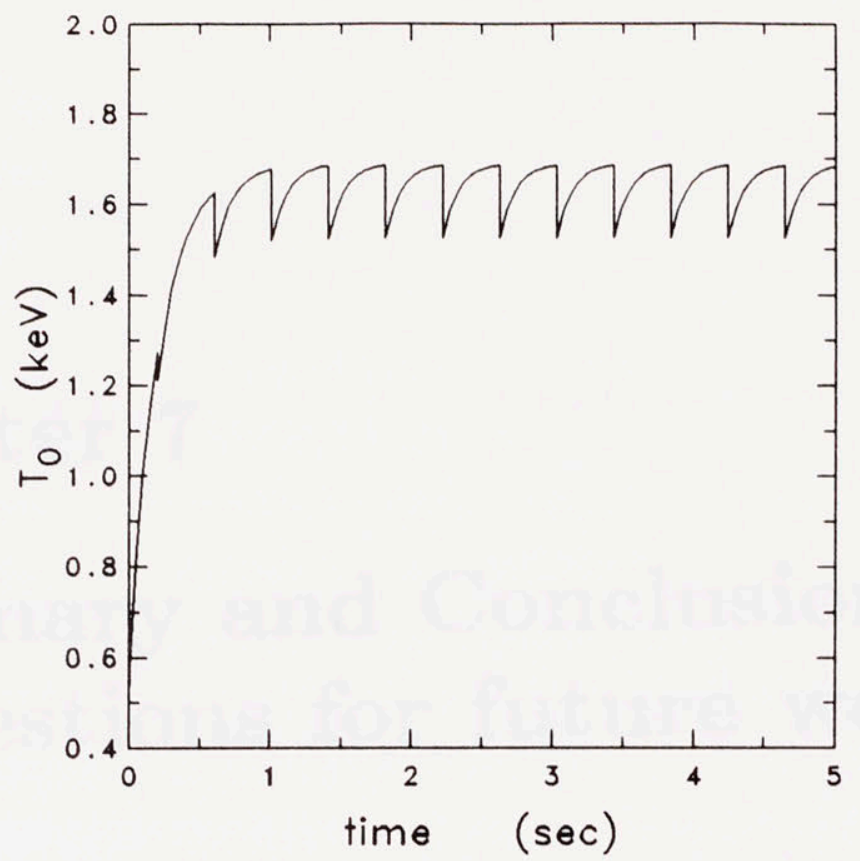

Figure 6.7: A plot of the peak temperature during sawtooth activity with a prescribed period corresponding to Fig. 6.6 


\section{Chapter 7}

\section{Summary and Conclusions; Suggestions for future work}

\subsection{Summary}

In this thesis issues related to ignition/design, burn control, and the development of a new transport model based on variational techniques have been investigated.

The various plasma transport models used in the analysis were reviewed in chapter 2 . There by starting with the Fokker-Planck equation the various assumptions leading to the $1 \frac{1}{2}-\mathrm{D}$, and eventually to the 0 -D transport models were presented, and the various models were cast in a form suitable for computation.

The procedure for designing optimized and ignited tokamaks was presented on chapter 3 . There, by introducing the the parameters $B \tau_{E} / R$, $n R / B$, and $R P_{a} / a^{2} B^{2}$ it was shown that $B \tau_{E} / R$ represents a universal performance parameter since it does not vary as various forms are assumed for the energy confinement time $\tau_{E}$. Next by explaining the importance of keeping the ratio $P_{a} / P_{\Omega}$ as small as possible, optimized designs were obtained by minimizing the ratio $P_{a} / P_{\Omega}$. The resulting optimized and ignited designs indicate the possible advantage of high magnetic field $\sim 15 \mathrm{~T}$ and high aspect ratio $\sim 4.5$. 
The method of plasma burn control via auxiliary power modulation was presented on chapter 4 with particular emphasis on the CIT design. There it was shown that auxiliary power can be a very important knob for tokamak operation. First, by modulating the auxiliary power it is possible to map the plasma operating space. Second, thermal equilibria can be created in the unstable regime by suitably reducing the auxiliary power supplied to the plasma. Third by modulating the auxiliary power about these equilibria negative and positive temperature perturbations can be effectively stabilized. Also the limits of operation of the auxiliary power control system were investigated. In particular it was found that operation with $Q \sim 50$ is possible, and that the feedback control system can be characterized with delay times in the order of 1 second. This delay time may be used in order to differentiate between fluctuations that require stabilization, and benign oscillations such as sawteeth.

The analysis presented in chapters 3 and 4 were performed via the 0 D plasma transport model. Since such a model is characterized by fixed profiles the need was recognized for the development of a plasma transport model which could provide profile information as fast as the 0 -D model to yield its results. In order to solve this problem the variational formulation was applied to the transport equation. In chapter 5 the basic principles of the variational formulation were formulated and examples of variational solutions to the general transport equation were presented. In particular the variational formulation was tested for the case of a single transport equation with non-linear phenomenological coefficients (including convection terms, and heat pulses) as well as for the case of a system of two coupled transport equations. In general, the variational formulation yields results by a factor of 10 - 50 faster than standard finite differencing schemes. Besides the increase in speed the solutions obtained with the variational formulation are accurate when compared with the exact solutions.

Having demonstrated the potential advantage of the variational formulation in obtaining solutions to the transport equations, the formalism was 
next generalized in chapter 6 for the system of $1 \frac{1}{2}-\mathrm{D}$ plasma transport equations developed in chapter 2. In particular the variational formulation was extended to a set of coupled equations describing the evolution of deuterium density, tritium density, alpha particle density, a single impurity species, the ion temperature, and the magnetic flux. The flux surface averaging required in the $1 \frac{1}{2}-\mathrm{D}$ model is obtained by solving the GradShafranov equation with the equilibrium solver developed by Haney [73]. In addition to the general transport model a sawtooth model was also developed. Some sample cases were presented which indicate the operation and the capabilities of the variational transport model. In particular sample ignition and burn control cases were presented for the CIT tokamak.

\subsection{Future Work}

The $1 \frac{1}{2}-\mathrm{D}$ variational transport model developed in this thesis can be used to perform a number of studies related to tokamak performance. The main advantage of this model is that profile information can be obtained in a time similar to that required by the standard $0-\mathrm{D}$ models. The burm control characteristics presented in chapter 4 were obtained by using the 0 -D model and thus did not include any profile information. Many of the issues related to ignition and burn control are sensitive to profile characteristics and thus their study requires a $1 \frac{1}{2}-\mathrm{D}$ model.

In particular the following issues are of importance and require further investigation with the $1 \frac{1}{2}-\mathrm{D}$ model.

\section{Sawteeth.}

In general a tokamak discharge is characterized by many local and global instabilities with the sawtooth oscillation being one of the most important from the point of ignition and burm control. During a sawtooth oscillation the profiles of the plasma temperature and density flatten and there is enhanced transport from the plasma interior. 
This makes ignition more difficult to achieve and places some important limits on the feedback system required to control the plasma burn. In chapter 4 the importance of the delay time $\tau_{d}$ was introduced and the connection to the possible sawtooth effects was mentioned. The feedback system has to be able to discriminate against benign plasma oscillations such as sawteeth. This point was also introduced in chapter 4. With the fast variational transport model developed in this thesis it is planned to study the effects of sawteeth-of various periods-on plasma ignition and burn control. For example, preliminary calculations indicate that an effective burn control system, based on auxiliary power modulation and operating in a sawtoothing plasma, will be characterized by $Q$ values that are lower than those characterizing a plasma without sawteeth. In the future, and with the aide of the variational transport model, these issues related to sawteeth effects will be investigated.

\section{Impurities}

Another important effect on ignition and burn control is the plasma impurity level. For example, since impurities tend to concentrate in the plasma center, it is important to model their transport in order to study their effect on both the ignition and burn control characteristics of the plasma.

Another important area that needs investigation is the effect of noncoronal impurity radiation on plasma control. Up to now plasma control and regulation studies, via impurity injection, has mainly concentrated on coronal equilibria. However, as the impurity ion average charge increases and approaches the coronal charge state distribution the radiated power decreases by one or two orders of magnitude.[74] Thereby it is possible by injecting light impurities into the plasma, and by including the radiated power due to non-coronal radiation to find that the actual amount of impurity injection required for burn 
control and regulation can be substantially decreased. The possible application of non-coronal radiation in the case of plasma shutdown, which is a special case of burn control, needs to be evaluated. For example, by inducing negative temperature perturbations and allowing them to grow in a controlled manner, the shutdown phase can be slow enough for the poloidal field system to follow the decrement of plasma pressure, thereby minimizing the possibility of disruptions. Another application of non-coronal radiation is for edge temperature control. Low temperatures are required in order to minimize erosion problems of the plasma facing components. Low temperatures can be achieved by injecting small amount of impurities near the divertor plates.

3. Transport assumptions

One of the most important considerations when using a $1 \frac{1}{2}-\mathrm{D}$ transport model is the type of transport assumptions used in the model. Since there is no agreement on the form of the plasma transport coefficients it is important to be able to perform parametric studies in order to cover the whole range of possible functional shapes and dependences. With the variational model developed in this thesis it is possible to investigate tokamak behavior under various transport assumptions by virtue of the fast execution of the model. In particular by evolving a tokamak plasma consistent with a particular global confinement scaling, according to Eq. (6.135), the effect of various transport coefficients can be investigated.

4. Off-axis heating

Controlling the plasma via auxiliary power modulation has been shown to be an effective way to stabilize temperature fluctuations. It is planned to extend the analysis with the aide of the new variational transport model in order to investigate the effect of various types of auxiliary power heating profiles. In particular it is important to quantify the response of the burn control system for situations in which the auxiliary power is deposited in the plasma periphery. 


\section{Diagnostic tool}

By virtue of its fast execution time, the variational transport model developed in this thesis can be used as a between shot-diagnostic in tokamak experiments in order to determine the various transport coefficients. For example, it is possible by measuring the profiles of density and temperature as well as the global energy confinement time, to deduce the profiles of the various diffusion coefficients.

6. Fueling

During pellet fueling the plasma undergoes density, temperature, and thus fusion power fluctuations. These fluctuations should not effect the burn control system and it is important to investigate the type of feedback system required in order to filter those types of fluctuations. In particular the feedback delay time has to be consistent with the time scale characterizing particle diffusion.

Finally, the variational transport model developed in this thesis is very suitable for use as a module in a more general tokamak systems code. A tokamak systems code which incorporates the variational transport model developed here, and the variational equilibrium solver developed by Haney [73], will be fast and also will provide important profile information. 


\section{Appendix A}

\section{Coefficients for the 0-D Transport Equations}

In this appendix the coefficients $K_{j}$, and $C_{j}$ appearing in the 0 -D equations are presented.

$$
\begin{aligned}
K_{l} & =\frac{1.2 \times 10^{5}}{1+\nu_{n}+\nu_{T}} \\
K_{\Omega} & =\frac{1.67 \times 10^{4}}{1+1.5 \nu_{T}}\left(\frac{1+\kappa^{2}}{\kappa}\right)^{2} Z_{\text {eff }} \\
K_{\alpha} & =\frac{5.6 \times 10^{5}}{\nu_{T}} \\
K_{b} & =\frac{5.3 \times 10^{3}}{1+2 \nu_{n}+.5 \nu_{T}} Z_{\text {eff }} \\
K_{a} & =\frac{5.07 \times 10^{4}}{\kappa} \\
K_{s} & =\frac{6.21 \times 10^{3}}{1+\nu_{n}+\nu_{T}} \\
C_{1} & =\frac{0.0507}{\kappa .5} \\
C_{2} & =\frac{0.14}{\nu_{T}}
\end{aligned}
$$




$$
\begin{aligned}
f & =\frac{n_{e, 0}}{\bar{n}_{e}} \approx\left(1+1.24 \nu_{n}\right)^{0.502} \\
C_{\Omega} & =0.3479 \frac{1+\nu_{n}+\nu_{T}}{1+1.5 \nu_{T}}\left(\frac{1+\kappa^{2}}{\kappa}\right)^{2} Z_{\text {eff }} \\
C_{\alpha} & =2.9167 \frac{1+\nu_{n}+\nu_{T}}{\nu_{T}} \\
C_{b} & =0.1104 \frac{1+\nu_{n}+\nu_{T}}{1+2 \nu_{n}+.5 \nu_{T}} Z_{\text {eff }} \\
C_{a} & =1.0563 \frac{1+\nu_{n}+\nu_{T}}{\kappa}
\end{aligned}
$$




\section{Bibliography}

[1] G. G. Dolgov-Savel'ev et al. Toroidal discharges in a strong magnetic field. Soviet Physics JETP, 11:287, 1960.

[2] L. A. Artsimovich and K. B. Kartashev. Effect of a transverse magnetic field on toroidal discharges. Soviet Physics-Doklady, 7:919, 1962.

[3] S. I. Braginskii. Transport Processes in a Plasma, volume 1 of Reviews of Plasma Physics, pages 205-311. Consultants Bureau, New York, 1965.

[4] J.P. Freidberg. Ideal Magnetohydrodynamics. Modern Perspectives in Energy. Plenum Press, New York, 1987.

[5] J. Wesson. Tokamaks. Oxford Science Publications, 1987.

[6] S. P. Hirshman and D. J. Sigmar. Neoclassical transport of impurities in tokamak plasmas. Nuclear Fusion, 21(9), 1981.

[7] F. L. Hinton and R. D. Hazeltine. Theory of plasma transport in toroidal confinement systems. Reviews of Modern Physics, 48(2), 1976.

[8] L. Spitzer. Physics of Fully Ionized Gases. Interscience, New York, second edition, 1962.

[9] D. Sigmar and J. Joyce. Nuclear Fusion, 11(3), 1971.

[10] T.J. Dolan. Fusion Research, volume 1. Pergamon Press, 1980. 
[11] I.H. Hutchinson. Principles of Plasma Diagnostics. Cambridge University Press, Cambridge, 1987.

[12] B.A. Trubnikov. Universal Coefficients for Synchrontron Emission from Plasma Configurations. Reviews of Modern Physics, 7:345, 1979.

[13] R.R. Parker et al. Progress in Tokamak Research at MIT. Nuclear Fusion, 25(3), 1985.

[14] R. Goldston. Energy Confinement Scaling for Tokamaks. Plasma Phus. Controlled fusion, 26:87, 1984.

[15] S. M. Kaye et. al. Global Energy Confinement Scaling for Neutral Beam Heated Tokamaks. Nuclear Fusion, 25:26, 1985.

[16] A. Cheetham et al. In Proceedings of the 12th IAEA Conference on Plasma Physics and Controlled Nuclear Fusion Research, volume 2, page 187, 1986.

[17] B.J.D. Tubbing et al. Nuclear Fusion, 27:1843, 1987.

[18] N.J.Lopes-Cardozo et al. Nuclear Fusion, 28:1173, 1988.

[19] R.J. Hawryluk et al. TFTR Plasma Regimes. In Plasma Physics and Controlled Nuclear Fusion Research, volume 1, pages 51-64. IAEA, 1987.

[20] F. Troyon et al. MHD limits to plasma confinement. Plasma physics and controlled fusion, 26:209, 1984.

[21] F. Troyon and R. Gruber. A semi-empirical scaling law for the $\beta$ limit in tokamaks. Physics Letters, 29:110, 1985.

[22] M. Murakami et al. Some Observations on Maximum Density in Tokamak Experiments. Nuclear Fusion, 16(2), 1976. 
[23] I.H. Hutchinson. Requirements for Ohmic Ignition. Journal of Fusion Energy, 6(3), 1987.

[24] W.A Houlberg, S.E Attenberger, and S.M. Hively. Nuclear Fusion, 2:935-945, 1982.

[25] L. Bromberg. Private Communication.

[26] J.A. Stratton. Electromagnetic Theory. McGraw-Hill, New York, 1941.

[27] B. D. Montgomery. Soleinoid Magnet Design. Robert E. Krieger Publishing Company, Huntington New York, 1980.

[28] ITER Physics Design Guidlines. Technical report, IAEA, Vienna, 1990.

[29] L. Grisham, S. Scott, and R. Goldston. Scaling of Confinement with Major Radious in TFTR. In Proceedings of the European Physical Society, Amsterdam, 1990.

[30] D.R. Cohn and L. Bromberg. Journal of Fusion Energy, 5:161, 1986.

[31] D.R. Cohn. Journal of Fusion Energy, 6:281, 1987.

[32] International Atomic Energy Egency. International Tokamak Reactor, Zero Phase, Vienna, 1980.

[33] C. Powell. Control of the Energy Balance in a Fusion Plasma. Plasma Physics, 15:1007, 1973.

[34] E. L. Vold T. K. Mau and R. W. Cohn. Tokamak power reactor ignition and time-dependant fractional power operation. Fusion Technology, 12:197, 1987.

[35] T. W. Petrie and J. M. Rawls. Burn control resulting from toroidal field ripple. Nuclear Fusion, 20:419, 1980. 
[36] T. W. Petrie and J. M. Rawls. Burn control via regulated ripple applied to reactor grade plasmas. Nuclear Fusion, 20:1461, 1980.

[37] T. E. Stringer. Effect of the magnetic field ripple on diffusion in tokamaks. Nuclear Fusion, 12:689, 1972.

[38] K. C. Shaing and J. D. Callen. Neoclassical ripple transport in tokamaks. Nuclear Fusion, 22:1061, 1982.

[39] L. Bromberg D. R. Cohn. Passive thermal-stability control in ignited tokamaks by ripple transport and radial motion. Nuclear Fusion, 21:201, 1981.

[40] A. Sestero. Control of burn-up phase. In G. Casini, editor, Plasma Physics for Thermonuclear Fusion Reactors, page 389. 1981.

[41] M. Ohnishi A. Saiki and M. Okamoto. Space-dependent analyis of feedback control to suppress thermal runaway by compressiondecompression. Nuclear Technology/Fusion, 5:326, 1984.

[42] S. V. Putvinskii. Stability of thermonuclear burn in a tokamak. Sov. J. Plasma Physics, 6:694, 1980.

[43] U Carretta E. Minardi and N. Bacelli. Plasma position control in a tokamak reactor around ignition. Nuclear Fusion, 26:599, 1986.

[44] L. Bromberg J. L. Fisher D. R. Cohn. Active burn control of ignited plasmas. Nuclear Fusion, 20(2), 1980.

[45] L. Harten V. Fuchs and A. Bers. Creating stable tokamak reactor equilibria by supplemental heating. Nuclear Fusion, 20:833, 1980.

[46] V. Fuchs L. Harten and A. Bers. On tokamak thermal equilibria and thermal stability. Nuclear Fusion, 20(5), 1980. 
[47] C. Lanczos. The Variational principles of Mechanics. Univ. of Toronto Press, Toronto, 1949.

[48] H. Goldstein. Classical Mechanics. Addison-Wesley, Reading, Massachusetts, 1950.

[49] R. Weinstock. Calculus of Variations. McGraw-Hill, New York, 1952.

[50] I. Prigogine. Evolution Criteria Variational Properties and Fluctuations. In Symposium on Non-Equilibrium Thermodynamics, chapter 1, pages 3-16. University of Chicago Press, 1965.

[51] P.M. Morse and H. Feshbach. Methods of Theoretical Physics. McGraw-Hill, New York, 1953.

[52] I. Prigogine. Bull. Acad. Belg. Cl. Sci., 31:600, 1954.

[53] L. D. Landau and e. M. Lifshitz. Statistical Physics. Addison-Wesley, Reading, Mass., 1958.

[54] P. Glansdorff, I. Prigogine, and D.F. Hayes. Variational properties of a viscous liquid at a nonuniform temperature. Physics of Fluids, 5:144-149, 1962.

[55] I. Prigogine. Introduction to Thermodynamics of Irreversible Processes. Wiley, New York, second edition, 1961.

[56] N.J. Peacock, D.C. Robinson, M.J. Forrest, P.D. Wilcock, and V.V Sannikov. Measurement of the Electron Temperature by Thomson Scattering in Tokamak T3. Nature, 224:488-490, 1969.

[57] S.C Jardin, N. Pomphrey, and J. DeLucia. Dynamic Modeling of Transport and Positional Control of Tokamaks. Journal of Computational Physics, 66:481-507, 1986. 
[58] C.E. Singer et al. Baldur: A One Dimensional Plasma Transport Code. Technical Report PPPL-2073, Princeton Plasma Physics Laboratory, 1986.

[59] W.A. Houlberg and R.W. Conn. Space Dependent Thermal Stability of Reacting Tokamak Plasmas. Nuclear Fusion, 19:81, 1979.

[60] R.J. Goldston. Journal of Computational Physics, 43, 1981.

[61] D.W. Ross et al. Thermal and Particle Transport in Tokamak Theoretical Models for Ignition Studies. IPSG panel report, 1987.

[62] von Goeler et al. Studies of internal disruptions and $\mathrm{m}=1$ oscillations in tokamak discharges with soft x-ray techniques. Physical Review Letters, 33:1201, 1974.

[63] B.B. Kodomtsev. Disruptive instability in tokamaks. Soviet Journal of Plasma Physics, 1:389-391, 1976.

[64] G.L. Jahns et al. Internal Disruptions in Tokamaks. Nuclear Fusion, 18:609-628, 1978.

[65] A. W. Edwards et al. Rapid collapse of a plasma sawtooth oscillation in the JET tokamak. Physical Review Letters, 57:210, 1986.

[66] B.V. Waddell et al. Nuclear Fusion, 18(5), 1978.

[67] W. Pfeiffer et al. Internal disruptions in tokamaks. Nuclear Fusion, 25:655, 1985.

[68] K. McGuire et al. In Proceedings of the 12th European Conference on Controlled Fusion and Plasma Physics, volume 1, page 134, 1985.

[69] D.J. Campbell et al. In Proceedings of the Twelfth European Conference on Controlled Fusion and Plasma Physics, volume 1, page 130, 1985. 
[70] R. Snider and A. Kellman. Scaling of the Sawtooth Inversion Radius and Mixing Radius on DIII-D. Anahaim California, 1989. APS.

[71] R. Petrasso et al. Nuclear Fusion, 21:881-889, 1981.

[72] H. Soltwisch. Measurement of current-density changes during the sawtooth activity ia a tokamak by far-infrared polarimetry. Rev. Sci. Instrum., 59:1599-1604, 1988.

[73] S.W. Haney. Methods for the Design and Optimization of Shaped Tokamaks. PhD thesis, Massachusetts Institute of Technology, 1988.

[74] P.G. Carolan and V.A. Piotrowicz. Plasma Physics, 25(10), 1983. 